\title{
Pancreatic $\beta$-cell specific loss of E2f1 impairs insulin secretion and $\beta$-cell identity through the epigenetic repression of non $\beta$-cell programs
}

Frédérik Oger ${ }^{1,7}$, Cyril Bourouh ${ }^{1,7}$, Xavier Gromada ${ }^{1}$, Maeva Moreno ${ }^{1}$, Charlène Carney ${ }^{1}$, Emilie Courty ${ }^{1}$, Nabil Rabhi ${ }^{1, \#}$, Emmanuelle Durand ${ }^{1}$, Souhila Amanzougarene ${ }^{1}$, Lionel Berberian ${ }^{1}$, Mehdi Derhourhi ${ }^{1}$, Laure Rolland ${ }^{1}$, Sarah Anissa Hannou ${ }^{1}$, Pierre-Damien Denechaud $^{2, \text { I , Zohra Benfodda }}{ }^{3}$, Patrick Meffre ${ }^{3}$, Lluis Fajas ${ }^{2}$, Julie Kerr-Conte ${ }^{4}$, François Pattou ${ }^{4}$, Philippe Froguel ${ }^{1,5}$, Amélie Bonnefond ${ }^{1}$ and Jean-Sébastien Annicotte ${ }^{1,6^{*}}$

${ }^{1}$ Univ. Lille, Inserm, CHU Lille, Institut Pasteur de Lille, CNRS, U1283 - UMR 8199 EGID, F-59000 Lille, France

${ }^{2}$ Center for Integrative Genomics, Université de Lausanne, CH-1015 Lausanne,

Switzerland

${ }^{3}$ Univ. Nîmes, EA7352 CHROME, F-30021 Nîmes cedex 1, France

${ }^{4}$ Univ. Lille, Inserm, CHU Lille, U1190 - EGID, F-59000 Lille, France

${ }^{5}$ Department of Metabolism, Imperial College London, Hammersmith Hospital, London W12 ONN, UK

${ }^{6}$ Lead contact

${ }^{7}$ Contributed equally to this work

\# Present address: Department of Biochemistry, Boston University School of Medicine, MA02118, USA

I Present address: Inserm, UMR1048, Institute of Metabolic and Cardiovascular Diseases, F-31000 Toulouse, France

*Correspondence: jean-sebastien.annicotte@inserm.fr (J.-S. A.)

INSERM U1283-EGID (INSERM / CNRS / Université de Lille / Institut Pasteur de Lille) Faculté de Médecine - Pôle recherche 1 place de Verdun - 59045 Lille Cedex - France

Phone: +33.(0)3 74008100

Running title: E2F1 and beta-cell identity 


\section{Abstract}

The loss of pancreatic $\beta$-cell identity emerges as an important feature of type 2 diabetes development, but the molecular mechanisms are still elusive. Here, we explore the cellautonomous role of the cell cycle regulator and transcription factor E2F1 in the maintenance of $\beta$-cell identity and insulin secretion. We show that the $\beta$-cell-specific loss of E2f1 function in mice triggers glucose intolerance associated with defective insulin secretion, an altered $\alpha$-to- $\beta$-cell ratio, a downregulation of many $\beta$-cell genes and a concomitant increase of non- $\beta$-cell markers. Mechanistically, the epigenomic profiling of non-beta cell upregulated gene promoters identified an enrichment of bivalent H3K4me3/H3K27me3 or H3K27me3 marks. Conversely, downregulated genes were enriched in active chromatin H3K4me3 and H3K27ac histone marks. We find that histone deacetylase inhibitors modulate E2F1 transcriptional and epigenomic signatures associated with these $\beta$-cell dysfunctions. Finally, the pharmacological inhibition of E2F transcriptional activity in human islets also impairs insulin secretion and the expression of $\beta$-cell identity genes. Our data suggest that E2F1 is critical for maintaining $\beta$-cell identity through a sustained repression of non $\beta$-cell transcriptional programs. 


\section{Introduction}

Type 2 Diabetes (T2D) is a progressive metabolic disorder characterized by permanent high blood glucose levels due to inadequate pancreatic $\beta$-cell response to peripheral insulin resistance. Normally, $\beta$-cells respond to obesity and aging associated insulin loss of sensibility by increasing insulin secretion to avoid rising glycemia. However, in case of imbalance between insulin secretion and action, the emerging chronic hyperglycemic state progressively leads to massive $\beta$-cell dysfunction and decreased mass (Weir \& Bonner-Weir, 2004). Importantly, single cell transcriptomic analysis of human pancreatic islet cells from diabetic and healthy individuals allowed the community to define specific gene signatures of endocrine cell types and revealed a complex multi cellular lineage identity of T2D (Segerstolpe et al, 2016; Wang et al, 2016; Xin et al, 2016). In addition, recent studies demonstrated a loss of transcriptional maturity (Avrahami et al, 2020) and cell-type-specific regulatory profiles underlying T2D (Rai et al, 2020). Moreover, histology studies of diabetic human pancreata found a significant increase in bi-hormonal Insulin+ (Ins+)/Glucagon+ (Glu+) cells suggesting an altered $\beta$-cell identity (Dor \& Glaser, 2013; Spijker et al, 2015; White et al, 2013). Accordingly, recent studies using $\beta$-cell lineage tracing murine models have demonstrated that islet cells have the capacity to directly trans-differentiate to another islet cell fate and/or de-differentiate to a progenitorlike cell (Brereton et al, 2014; Spijker et al, 2013; Spijker et al., 2015; Talchai et al, 2012). In this context, both genetic and epigenetic mechanisms are important to maintain adult $\beta$ cell fate and function in mouse and human (Bramswig et al, 2013; Chakravarthy et al, 2017; Collombat et al, 2009; Ediger et al, 2017; Gutierrez et al, 2017; Lawlor \& Stitzel, 2019; Lu et al, 2018; Schaffer et al, 2013; Thorel et al, 2010; Yang et al, 2011). Yet, the molecular actors controlling $\beta$-cell mass, identity maintenance and cellular plasticity remain poorly understood.

Gene transcription and chromatin states are tightly regulated to ensure the appropriate transcriptome for a specific cell type. Many transcription factors have been identified as key regulators of $\beta$-cell identity and function, including Pdx1 (Gao et al, 2014), Pax6 (Swisa et al, 2017), Nkx6.1 (Schaffer et al., 2013) and Nkx2.2 (Gutierrez et al., 2017). Interestingly, pleiotropic transcription factors are also involved in the control of $\beta$ cell functions and glucose homeostasis, suggesting their important roles in activating or repressing gene transcription. Amongst those, members of E2F transcription factors (E2F1 to E2F8) family play critical roles in cell survival and proliferation, by regulating the gene expression of several proteins involved in cell-cycle progression (Kent \& Leone, 2019; 
Poppy Roworth et al, 2015). The transcriptional activity of E2F1, the founder member of the family, is regulated by several protein complexes including the retinoblastoma tumor suppressor family (pRB, p107, p130), cyclin-dependent kinases (such as CDK4) and their regulatory partner cyclins (Ccn), as well as the family of the cdk inhibitors (CDKi, such as p16 ${ }^{\text {Ink4A }}$ encoded by the Cdkn2a locus) (Poppy Roworth et al., 2015). Interestingly, the role of the cell cycle machinery goes beyond the unique regulation of cell proliferation. Indeed, modulating the expression levels of these cell cycle regulators revealed an important role for these proteins in glucose homeostasis (Denechaud et al, 2017) and diabetes development through the control of $\beta$-cell mass (Salas et al, 2014; Wang et al, 2015). Although E2F1 gene expression is decreased in human T2D islets (Lupi et al, 2008), the causal effect of E2F1 deficiency on impaired $\beta$-cell mass, function and T2D development is not elucidated. In particular, the cellular and molecular mechanisms underlying the contribution of E2F1 as a transcription factor to $\beta$-cell identity and/or plasticity in mice and humans remain unknown. We recently demonstrated that the germline deletion of E2f1 in the obese and diabetic $d b / d b$ mouse model, despite lowering liver steatosis, does not protect against diabetes or obesity (Denechaud et al, 2016). Interestingly, we observed decreased plasma insulin levels, increased plasma glucose and glucose intolerance in $d b / d b:: E 2 f 1^{-/}$mice compared to $d b / d b:: E 2 f 1^{+/+}$controls (Denechaud et al., 2016). These metabolic alterations in a diabetic background raises the possibility that E2f1 may contribute to islet morphology, cell identity and function in a cell-autonomous manner. To test this hypothesis, we generated mice lacking E2f1 in $\beta$ cells and identified that E2F1 is necessary for maintaining $\beta$-cell identity gene expression in both mouse models and human islets. By combining cellular and mouse models to pharmacological approaches, our results identify E2F1 as a critical transcription factor necessary to maintain proper $\beta$ cell gene expression and function, while repressing non $\beta$-cell transcriptional programs. 


\section{Material and methods}

Materials and Oligonucleotides. Chemicals, unless stated otherwise, were purchased from Sigma-Aldrich. Anti-insulin (ab7842), anti-glucagon (ab11909) and IgG (ab37415 ChIP grade) antibodies for ChIP-seq experiments were from Abcam ; anti-E2F1 (sc-193 for immunofluorescence on mouse tissues) antibody was from Santa Cruz Biotechnology. H3K4me3 (\#61379), H3K27ac (\#39685) and H3K27me3 (\#61017) antibodies were from Active motif. The HLM006474 compound (Ma et al, 2008) was synthetized as previously described (Rosales-Hurtado et al, 2019).

Animal Experiments. Mice were maintained according to European Union guidelines for the use of laboratory animals. In vivo experiments were performed in compliance with the French ethical guidelines for studies on experimental animals (animal house agreement no. A 59-35015, Authorization for Animal Experimentation no.59-350294, project approval by our local ethical committee no. CEEA 482012, APAFIS\#2915-201511300923025v4). All experiments were performed with male mice. Mice were housed under a 12-hr light/dark cycle and given a regular chow (A04;Safe).

E2f1-/- (B6; 129S4-E2f1tm1Meg/J) mice and db/+ mice (Janvier Labs) were crossed to obtain $d b / d b:: E 2 f 1^{+/+}$and $d b / d b:: E 2 f 1^{-/-}$mice and were previously described in (Denechaud et al., 2016). CMV-CDK4 $4^{R 24 C}$ (Sotillo et al, 2001) and Cdkn2a-/- (Serrano et al, 1996) were described elsewhere and were crossed with E2f1-/- mice to obtain E2f1-/$\therefore$ CMV-CDK4 ${ }^{\text {R24C }}$ and E2f1-/-::Cdkn2a-/-. E2f1 floxed (E2f1 flox/flox, Taconic Biosciences, NY, USA) mice were previously described (Denechaud et al., 2016; Giralt et al, 2018). The congenic mice carrying the floxed E2f1 allele were thereafter mated with rat insulin II promoter (RIP)-Cre mice (Herrera, 2000) and then further intercrossed to generate pure mutant RIPcre ${ }^{T g /+}:$ :E2f1 ${ }^{\text {flox/flox }}$ mice. A PCR genotyping strategy was subsequently used to

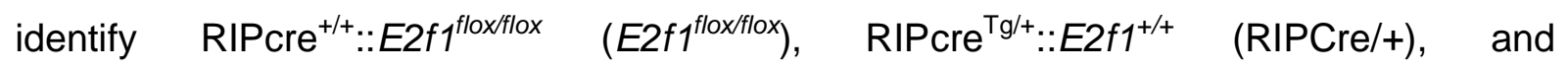
RIPcre ${ }^{T g /+}:: E 2 f 1^{\text {flox/flox }}\left(E 2 f 1^{\beta-/}\right)$ mice.

Metabolic phenotyping experiments were performed according to the EMPRESS protocols. Intraperitoneal glucose and insulin tolerance tests (ipGTT and ITT, respectively) were performed as previously described (Annicotte et al, 2009; Rabhi et al, 2016) on 16hr-fasted animals for ipGTT and 5-hr-fasted animals for ITT. Glycemia was measured using the Accu-Check Performa (Roche Diagnostics). Circulating insulin levels were measured using the mouse Insulin ELISA kit (Mercodia). 


\section{Immunofluorescence, Immunohistochemistry and Morphometry.}

Immunofluorescence and immunohistochemistry were performed exactly as described previously (Annicotte et al., 2009; Rabhi et al., 2016). Pancreatic tissues were fixed in 10\% formalin, embedded in paraffin and sectioned at $5 \mu \mathrm{m}$. For immunofluorescence microscopy analyses, after antigen retrieval using citrate buffer, 5- $\mu \mathrm{m}$ formalin-fixed paraffin embedded (FFPE) pancreatic sections were incubated with the indicated antibodies. Immunofluorescence staining was revealed by using a fluoresceinisothiocyanate-conjugated anti-rabbit (for E2f1; Santa Cruz), Alexa-conjugated anti-mouse (for glucagon co-staining with E2f1) or anti-guinea pig (for insulin co-staining with E2f1) secondary antibodies. Nuclei were stained with Hoechst. For morphometric analysis, three to ten animals from each genotype were analyzed, and images were processed and quantified using ImageJ software by an observer blinded to experimental groups.

Pancreatic Islet Studies. For mouse islet studies, pancreata were digested by type $\mathrm{V}$ collagenase (C9263; $1.5 \mathrm{mg} / \mathrm{ml}$ ) for $10 \mathrm{~min}$ at $37^{\circ} \mathrm{C}$ as described previously (Rabhi et al., 2016). Briefly, after digestion and separation in a density gradient medium, islets were purified by handpicking under a macroscope and cultured during 16 hours before subsequent analysis. For glucose-stimulated insulin secretion (GSIS) tests, approximately twenty islets were exposed to either $2.8 \mathrm{mM}$ or $20 \mathrm{mM}$ glucose in Krebs-Ringer bicarbonate HEPES buffer containing $0.5 \%$ fatty-acid-free Bovine Serum Albumin (BSA). Insulin released in the medium was measured $1 \mathrm{hr}$ later using the mouse insulin ELISA kit (Mercodia). For expression studies, mouse isolated islets were snap-frozen in liquid nitrogen before RNA extraction. Human pancreatic tissue was harvested from brain-dead, non-diabetic adult human donors (see Supplementary Table S1 for donor information). Isolation and islets culture were performed as described elsewhere (Kerr-Conte et al, 2010). Human islets were treated for 48 hours with DMSO $0.1 \%$ or HLM006474 at $10 \mu \mathrm{M}$. Data are expressed as a ratio of total insulin content. For mRNA and protein quantification, human islets were isolated as described above and snap-frozen for further processing.

Cell Culture and Pharmacological Treatments. Min6 cells (AddexBio) were cultured in DMEM (Gibco) with $15 \%$ fetal bovine serum, $100 \mathrm{mg} / \mathrm{ml}$ penicillin-streptomycin, and 55 $\mathrm{mM}$ beta-mercaptoethanol. Cells were treated with Trichostatin A (TSA) at $0.5 \mu \mathrm{M}$ and subjected to GSIS or RNA extraction $16 \mathrm{hr}$ later. Min6 cells were treated with HLM006474 $(10 \mu \mathrm{M})$ or DMSO 0.1\% (Ma et al., 2008; Rosales-Hurtado et al., 2019) for $48 \mathrm{hr}$ before GSIS assay or RNA extraction. 
RNA extraction, qPCR and RNA-Sequencing. Total RNA was extracted from cells and tissues using trizol reagent (Life Technologies) as described previously. mRNA expression was measured after reverse transcription by quantitative real-time PCR (qRT-PCR) with FastStart SYBR Green master mix (Roche) using a LightCycler Nano or LC480 instruments (Roche). qRT-PCR results were normalized to endogenous cyclophilin reference mRNA levels. The results are expressed as the relative mRNA level of a specific gene expression using the formula $2^{-\Delta C t}$. The complete list of primers is presented in Supplementary Table S2. For RNA sequencing, total RNA was extracted from Min6 cells or pancreatic islets using the RNeasy Plus Microkit (Qiagen) following manufacturer's instructions. RNA quality was verified using RNA 6000 nanochips on the Agilent 2100 bioanalyzer. Purified RNA (50ng) with RNA integrity number $\geq 6.5$ was subsequently used for library preparation (TruSeq Stranded mRNA Library Preparation Kit, Illumina) and sequenced on a HiSeq2500 system (Illumina). 3 biological replicates per condition were sequenced using paired-end mode. A mean of 54 million paired-end reads of 75 bp were generated for each sample. After initial checks and validation of sequence quality, RNAseq reads were aligned to the mouse reference genome (mm10) using TopHat2. Subsequently, both quantification and annotation of the reads were performed using Bioconductor package Rsubread. Finally, the differential gene expression analyses were performed using Bioconductor package DESeq2. Using a $P<0.05$ adjusted for multiple comparisons as threshold, we then performed pathway analysis using Ingenuity Pathway Analysis (Ingenuity Systems, Qiagen), Metascape (Zhou et al, 2019) and Gene Set Enrichment Analysis (GSEA; http://software.broadinstitute.org/gsea/).

Chromatin immunoprecipitation and ChIP sequencing. ChIP experiments were performed on formaldehyde-fixed Min6 cells. Briefly, $20.10^{6}$ Min6 cells were treated with formaldehyde at a final concentration of $1 \%$ to crosslink DNA and protein complexes during $10 \mathrm{~min}$. The reaction was stopped by the addition of glycine $(0.125 \mathrm{M})$ during $5 \mathrm{~min}$. Cells were lysed and DNA-protein complexes were sheared using the Bioruptor Pico (Diagenode, ref B01060010) for 8 minutes. The sheared chromatin was immunoprecipitated with either the non-specific antibody IgG (Santa Cruz, sc2025), H3K4me3 (Active motif, \#61379), H3K27me3 (Active motif, \#61017) or H3K27ac (Active motif, \#39685). $1 \mathrm{ng}$ of eluted and purified DNA was used to prepare DNA sequencing library with the Nextflex rapid DNA seq kit 2.0 (Perkin Elmer, NOVA-5188-01) on the NextSeq 500 system (Illumina) using single read 100 base pairs mode. The demultiplexing 
of sequence data (from BCL files generated by Illumina sequencing systems to standard FASTQ file formats) was performed using bcl2fastq Conversion Software (Illumina; version 2.20). Trimming of residuals adapters and low quality reads was performed using TrimGalore (version 0.4.5). Subsequently, sequence reads from FASTQ files were mapped to the mouse genome (mm10) using Bowtie2 Aligner (version 2.3.5.1). Finally, peak-calling was performed with MACS2 software (version 2.2.7.1). Further bioinformatic analyses of the public data sets and those of this study were performed using the open web-based platform Galaxy Europe (https://usegalaxy.eu). A list of ChIP-seq and RNA-seq data sets used in this study can be found in Supplementary Table S3.

\section{Rapid immunoprecipation mass spectometry of endogenous protein (RIME).}

Chromatin Immunoprecipitation: Min6 cells were transfected with an empty vector (pCMVFLAG, negative control) or a vector encoding human E2F1 gene coupled to a FLAG tag for immunoprecipitation (pCMV-hE2F1-FLAG). 48h after transfections, cells were fixed in $1 \%$ formaldehyde for $15 \mathrm{~min}$ and quenched with $0.125 \mathrm{M}$ glycine. Chromatin was isolated by the addition of lysis buffer, followed by disruption with a Dounce homogenizer. Lysates were sonicated and the DNA sheared to an average length of 300-500 bp. Genomic DNA (Input) was prepared by treating aliquots of chromatin with RNase, proteinase $\mathrm{K}$ and heat for de-crosslinking, followed by ethanol precipitation. Pellets were resuspended and the resulting DNA was quantified on a NanoDrop spectrophotometer. Extrapolation to the original chromatin volume allowed quantitation of the total chromatin yield. An aliquot of chromatin (150 ug) was precleared with protein G agarose beads (Invitrogen). Proteins of interest were immunoprecipitated using 15 ug of antibody against FLAG (\#F9291) and protein $\mathrm{G}$ magnetic beads. Protein complexes were washed then trypsin was used to remove the immunoprecipitate from beads and digested the protein sample. Protein digests were separated from the beads and purified using a C18 spin column (Harvard Apparatus). The peptides were vacuum dried using a speedvac.

Mass Spectrometry: Digested peptides were analyzed by LC-MS/MS on a Thermo Scientific Q Exactive Orbitrap Mass spectrometer in conjunction with a Proxeon Easy-nLC II HPLC (Thermo Scientific) and Proxeon nanospray source. The digested peptides were loaded on a 100 micron x 25 mm Magic C18 100Å 5U reverse phase trap where they were desalted online before being separated using a 75 micron x 150 mm Magic C18 200A 3U reverse phase column. Peptides were eluted using a 90 minutes gradient with a flow rate of $300 \mathrm{nl} / \mathrm{min}$. An MS survey scan was obtained for the $\mathrm{m} / \mathrm{z}$ range $300-1600, \mathrm{MS} / \mathrm{MS}$ spectra were acquired using a top 15 method, where the top 15 ions in the MS spectra 
were subjected to HCD (High Energy Collisional Dissociation). An isolation mass window of $1.6 \mathrm{~m} / \mathrm{z}$ was for the precursor ion selection, and normalized collision energy of $27 \%$ was used for fragmentation. A five second duration was used for the dynamic exclusion.

Database Searching:_Tandem mass spectra were extracted by Unspecified version Unspecified. Charge state deconvolution and deisotoping were not performed. All MS/MS samples were analyzed using $\mathrm{X}$ ! Tandem (The GPM, thegpm.org; version CYCLONE (2013.02.01.1)). X! Tandem was set up to search the mouse-F-_20150428_KKOvMm database (unknown version, 90758 entries) the cRAP database of common laboratory contaminants (www.thegpm.org/crap; 114 entries) plus an equal number of reverse protein sequences assuming the digestion enzyme trypsin. $\mathrm{X}$ ! Tandem was searched with a fragment ion mass tolerance of 20 PPM and a parent ion tolerance of 20 PPM. Carbamidomethyl of cysteine was specified in X! Tandem as a fixed modification. Glu>pyro-Glu of the n-terminus, ammonia-loss of the n-terminus, gln->pyro-Glu of the $n$ terminus, deamidated of asparagine and glutamine, oxidation of methionine and tryptophan, dioxidation of methionine and tryptophan and acetyl of the n-terminus were specified in $\mathrm{X}$ ! Tandem as variable modifications.

Criteria for Protein Identification: Scaffold (version Scaffold_4.5.3, Proteome Software Inc., Portland, OR) was used to validate MS/MS based peptide and protein identifications. Peptide identifications were accepted if they exceeded specific database search engine thresholds. X! Tandem identifications required at least -Log(Expect Scores) scores of greater than 1.5. Protein identifications were accepted if they contained at least 1 identified peptides. Proteins that contained similar peptides and could not be differentiated based on MS/MS analysis alone were grouped to satisfy the principles of parsimony. Proteins sharing significant peptide evidence were grouped into clusters.

List Filtering: Final list generation was done by taking all proteins with a spectral count of five and above from each replicate reaction and comparing them in a venn-diagram against IgG control replicates. Proteins unique to both experimental replicates were then applied to the PANTHER database for protein ontology results.

Statistical Analysis. Data are presented as mean \pm s.e.m. Data are derived from multiple experiments unless stated otherwise. Statistical analysis was performed using a two-tailed unpaired $t$-test or one-way or two-way ANOVA with Tukey's post hoc test comparing all groups to each other, using GraphPad Prism 7.0 software. Differences were considered statistically significant at $p 0.05\left({ }^{*} p<0.05,{ }^{* *} p<0.01\right.$, and $\left.{ }^{* *} p<0.001\right)$. 


\section{Results}

\section{Increased alpha to beta cell ratio in the pancreas of germline E2f1-deficient mice.}

To investigate the contribution of E2f1 to islet morphology, we performed immunofluorescence staining of insulin and glucagon in the pancreas of wild-type and global E2f1 knock-out mice. The detailed analysis of E2f1-deficient pancreas revealed a decreased proportion of ins+ $\beta$-cell and a concomitant expansion of the glu+ $\alpha$-cell percentage per islet in chow-fed 16 week-old animals, as demonstrated through the quantification of insulin and glucagon positive cells in E2f1+/+ and E2f1-/- pancreas (Figures 1A-B, Supplementary figures S1G). In the $d b / d b$ background, E2f1 deficient animals displayed a further increase of the $\alpha$-cell number per islet, while exhibiting an even lower $\beta$-cell count compared to $d b / d b:: E 2 f 1^{+/+}$controls (Figures 1 C-D).

To test whether bona fide regulators of E2F1 activity could rescue the altered $\alpha$-to- $\beta$-cell ratio observed in E2f1 -/- mice, we used 2 different genetically-engineered mouse models (Supplementary figures S1A). First, the R24C mouse model of CDK4 hyperactivation $\left(\mathrm{CMV}-\mathrm{CDK} 4^{\mathrm{R} 24 \mathrm{C}}\right)$ constitutively expresses a mutant CDK4 protein that restores $\beta$-cell mass and function during diabetes development (Martin et al, 2003; Miyawaki et al, 2008; Rane et al, 1999). As previously observed (Rane et al., 1999), $\alpha$-to- $\beta$-cell ratio was decreased in $C M V-C d k 4^{R 24 C}$ pancreas (Supplementary figures S1B, S1C and S1G). We then generated compound mutant mouse models with both E2f1 deficiency and overactive CDK4 (E2f1-/$\left.\therefore C M V-C d k 4^{R 24 C}\right)$. Interestingly, the increased $\beta$-cell number observed in $C M V-C d k 4^{R 24 C}$ pancreas was blunted in E2f1-/-::CMV-Cdk4 ${ }^{R 24 C}$ mice, with a concomitant increase of glucagon immunofluorescent staining (Supplementary figures S1D and S1G). We replicated these data in the second model using Cdkn2a-deficient mice (Cdkn2a-/;(Serrano et al., 1996)), an upstream regulator of the E2F1-CDK4-pRb signaling pathway involved in $\beta$-cell function (Helman et al, 2016; Ndiaye et al, 2017; Pal et al, 2016). Although Glu+ and Ins+ immunofluorescent positive cell staining was conserved between Cdkn2a+/+ and Cdkn2a-/- pancreas (Supplementary figures S1E and S1G), E2f1-/$:: C d k n 2 a-/-$ compound mutant mice displayed an altered $\alpha$-to- $\beta$-cell ratio with a decrease of Ins+ and an increase Glu+ cell numbers, respectively (Supplementary figures S1F and $\mathrm{S} 1 \mathrm{G})$. Altogether, these results suggest a specific role of E2f1 in maintaining pancreatic $\beta$ cell numbers under normal conditions but also in a diabetic environment associated to glucose intolerance, insulin resistance and obesity. 
B-cell specific E2f1 deficiency impairs glucose tolerance, insulin secretion and alters $\alpha$-to- $\beta$ cell ratio.

To determine whether E2f1 regulates insulin-producing $\beta$-cell fate and function in a cellautonomous manner, we generated $\beta$-cell specific E2f1 deficient mice by crossing E2f1 floxed $\left(E 2 f 1^{f / f t}\right)$ with RIP-Cre (Rip-Cre/+) mice (Herrera, 2000). Quantitative RT-PCR showed a $91 \%$ tissue-specific reduction in E2f1 expression in pancreatic islets isolated from $E 2 f 1^{\beta-l-}$ mice (Supplementary figure S2A). $\beta$-cell specific deletion of E2f1 was also confirmed at the protein level by immunofluorescence analysis (Supplementary figure $\mathrm{S} 2 \mathrm{~B}$ ). $E 2 f 1^{\beta-/-}$ mice had normal body weight (Supplementary figure S2C) and fasting glycemia when fed a chow diet (Supplementary figure S2D). When challenged with a bolus of glucose, E2f1 ${ }^{\beta-/-}$ mice exhibit glucose intolerance (Figures $2 \mathrm{~A}-\mathrm{B}$ ), primarily due to decreased insulin secretion in response to glucose (Figure 2C) rather than defective insulin sensitivity (Figure 2D). GSIS experiments on mouse pancreatic islets isolated from $E 2 f 1^{f / f l}$ and $E 2 f 1^{\beta-/-}$ further confirmed insulin secretion defects in response to glucose (Figure 2E). Interestingly, an immunofluorescence analysis of sections from control and $E 2 f 1^{\beta-/-}$ pancreas demonstrated increased glucagon-positive cells and an increased $\alpha$-to- $\beta$ cell ratio in $\beta$-cell specific E2f1 KO islets (Figures 2F-G). The number of cells per islet section remained unchanged between control and $E 2 f 1^{\beta-/-}$ pancreas (Supplementary figure S2E). As observed in germline E2f1 deficient mice (Figure 1), these data suggest that $\beta$ cell expression of E2f1 is necessary to maintain insulin secretion and glucose homeostasis associated to normal $\alpha$-to- $\beta$-cell ratio.

\section{Loss of $\beta$-cell identity markers in E2f1 deficient pancreatic islets}

E2F1 is a transcription factor that controls gene expression in several cellular systems. In order to get a global view of the transcriptional mechanisms associated with the loss of E2f1 expression in the $\beta$ cell, we performed RNA sequencing (RNA-seq) in control $\left(E 2 f 1^{f / f f}\right)$ and $E 2 f 1^{\beta-/}$ isolated islets. As expected, the floxed region of the E2f1 gene spanning exon 2 and 3 was not covered in $E 2 f 1^{\beta-/-}$ isolated islets compared to E2f1 ${ }^{f / f l}$ isolated islets, indicating specific and efficient gene deletion through the Cre recombinase activity in $E 2 f 1^{\beta-/-}$ isolated islets (Supplementary figure S3A). The analysis of the transcripts revealed that 692 annotated genes were differentially expressed across the two groups (adjusted $P$ Value $(\mathrm{Adj} P)<0.05)$. Interestingly, a vast majority of the genes were upregulated in $E 2 f^{\beta-/}$ isolated islets (493 genes, Figure $3 A$ and Supplementary Table S4), 
with only 199 down-regulated genes associated with the loss of E2f1 expression in $\beta$ cells (Figure 3A and Supplementary Table S4). This first observation suggests that E2f1 expression is not only necessary to activate, but also to repress gene transcription within the $\beta$ cell. Analysis of the RNA-seq data of downregulated genes with metascape software revealed an enrichment of gene networks involved in signal release, regulation of exocytosis and negative regulation of secretion (Figure 3B). Conversely, upregulated genes in $E 2 \mathrm{ft}^{\mathrm{\beta}-\mathrm{-}}$ isolated islets were associated with the regulation of cell adhesion, inflammatory response and cytokine production (Figure 3C). To better understand the relationship between the observed metabolic phenotype (i.e. glucose intolerance and defective insulin secretion), increased glu+ cells, decreased ins+ cells and E2f1 deficiency, we filtered gene sets to focus on $\beta$ - and $\alpha$-cell genes being conserved between zebrafish, mouse and human (Tarifeno-Saldivia et al, 2017). Notably, a total of 15 genes from 109 conserved genes (82 and 27 for $\alpha$-cell and $\beta$-cell genes, respectively) were differentially expressed. Interestingly, most of the conserved $\beta$-cell genes were found to be decreased in $E 2 f^{\beta-/-}$ isolated islets (Figure 3D and Supplementary figure S3B), whereas most of the conserved $\alpha$-cell genes were upregulated upon E2f1 deficiency (Figure 3D and Supplementary figure S3C). Gene Set Enrichment Analysis (GSEA (Subramanian et al, 2005)) further confirmed an enriched signature of decreased expression of $\beta$-cell markers (Figure 3E) and conversely, an increased expression of $\alpha$-cell markers in $E 2 f 1^{\beta-/-}$ isolated islets (Figure 3F). We further confirmed these changes in the expression of selected $\alpha$ and- $\beta$-cell markers at the transcriptional level by qPCR in an independent experiment: indeed we observed a strong decrease in transcript levels of $P d x 1$, Mafa $(p=0.13)$, Ins2, Pcsk9, Foxo1 and Glp1r in E2f1 ${ }^{\beta-1-}$ islets (Supplementary figure S3D). Conversely, $\alpha$-cell specific Aristaless-related homeobox (Arx) mRNA levels were increased in $E 2 f 1^{\beta-/-}$ islets (Supplementary figure S3E). Altogether these results suggest that the $\beta$-cell specific deletion of E2f1 induces a transcriptional reprogramming characterized by a loss of $\beta$-cell identity genes associated to an increased expression of non $\beta$-cells markers.

\section{The treatment with the E2F inhibitor HLM006474 inhibits glucose-stimulated insulin secretion and impairs $\beta$ - and $\alpha$-cell gene expression in Min6 cells and human islets.}

To assess the effect of E2F1 activity on $\beta$-cell identity markers in human islets, we made use of the E2F pan-inhibitor HLM006474 previously shown to inhibit the binding of E2Fs to their DNA target genes (Sangwan et al, 2012) and E2F1 transcriptional activity (RosalesHurtado et al., 2019). Consistent with our previous findings in HEK293 cells (Rosales- 
Hurtado et al., 2019), a 48h treatment of Min6 cells with this inhibitor triggered a decrease in E2f1 transcriptional activity, as measured by transient transfection experiments using an E2F reporter gene (Figure 4A). In addition, the treatment of Min6 cells with this inhibitor induced a marked decrease in glucose-stimulated insulin secretion (Figure 4B) and in the expression of several $\beta$-cell markers, including Ins1, Pdx1, Pax4 and Nkx2.2 (Figure 4C). The treatment of human islets with the E2F inhibitor for 48 hours also decreased GSIS (Figure 4D) and $\beta$-cell marker expression levels with a concomitant increase in the expression of $\alpha$-cell genes (Figure 4E). Therefore, the pharmacological inhibition of E2F activity impairs $\beta$-cell function and gene expression in both mouse cell line and human islets, suggesting that E2F1 activity is also required in human islets to maintain proper insulin secretion and $\beta$-cell identity genes, as observed in mice.

\section{Maintenance of $\beta$-cell identity is dependent on histone deacetylase and E2F1 activities.}

Our transcriptome analysis revealed a 2.5-fold more up- than downregulation of global gene expression in $E 2 f 1^{\beta-/-}$ isolated islets. Comparative analysis of log 10 transcription levels $(\log 10(\mathrm{TPM}+1))$ of up- and downregulated genes values demonstrated that the expression of upregulated genes in $E 2 f 1^{\beta-/-}$ islets was significantly increased upon E2f1 deficiency compared to wild-type controls whereas the expression of downregulated genes was not significantly modulated (Figure 5A). Considering that E2f1 could play a dual role in the regulation of gene expression in pancreatic $\beta$ cells, we postulated that this mechanism could be related to a distinct epigenomic profile within promoter of genes that are up- and down-regulated in $E 2 f 1^{\beta-/-}$ islets. Using a recently published chromatin-state segmentation model (Lu et al., 2018), we probed the activation/repressive level of these promoters by monitoring several epigenome marks such as active and poised promoters (tri-methylation of lysine 4 in histone H3 [H3K4me3]) and enhancers (acetylation of lysine 27 in histone H3 [H3K27ac]). Intersecting publicly available data of chromatin immunoprecipitation followed by next-generation sequencing (ChIP-seq) from healthy C57BI6/J mouse pancreatic islets (GSE 110648 (Lu et al., 2018)) and our RNA-seq data, we grouped up- and down regulated genes according to their chromatin state (Figure 5B and Supplementary Table S4). $61 \%$ of the upregulated genes in $E 2 f 1^{\beta-/-}$ pancreatic islets were associated to a silent chromatin state in healthy $\mathrm{C} 57 \mathrm{BI} / \mathrm{J}$ mouse pancreatic islets characterized by bivalent H3K4me3/H3K27me3 and Polycomb-repressed (H3K27me3) marks. Conversely, 82\% of 
the down-regulated genes showed an enrichment in active chromatin state characterized by RNA-Pol2 recruitment, H3K4me3 and H3K27ac histone marks.

While H3K4me3, H3K27ac and H3K27me3 ChIP-seq data were available for mouse islets, we then performed ChIP-seq experiments in Min6 cells as a surrogate of $\beta$ cell. H3K4me3, H3K27ac and H3K27me3 ChIP-seq signals were thus interrogated within promoter (centered to transcription start site [TSS] +/- $1 \mathrm{~kb}$ ) of up- and downregulated genes both in Min6 cells and mouse pancreatic islets (Figures 5C-D and Supplementary figures 4A-B). $\mathrm{H} 3 \mathrm{~K} 4 \mathrm{me} 3$ as well as H3K27ac signals were stronger within promoter of genes that are downregulated in $E 2 f 1^{\beta-/-}$ isolated islets compared to upregulated genes, both in Min6 cells and mouse pancreatic islets (Figure 5C and Supplementary figures 4A-B). Conversely, H3K27me3 ChIP-seq signals were lower in the promoter region of downregulated genes compared to upregulated genes (Figure 5D and Supplementary figures 4A-B). We then analyzed our integrated RNA-seq/ChIP-seq data using the "upstream regulator analysis" function of Ingenuity Pathway Analysis (IPA) to identify potential contributors that could associate to the phenotype of $E 2 f 1^{\beta-/-}$ isolated islets, which would be related to epigenomics. Among the most significant upstream regulators of the upregulated genes, the HDAC inhibitor trichostatin A (TSA) signaling pathway was predicted to be significantly activated (Table 1), suggesting that HDAC could regulate an E2f1-dependent gene program. Consequently, having shown that $(i)$ the levels of upregulated genes were significantly increased upon $\beta$-cell specific E2f1 deletion (Figure 5A), (ii) H3K27ac signal was weaker within the promoter region of upregulated genes compared to downregulated genes (Figure 5C) and (iii) TSA may modulate the E2f1-dependent epigenome, we next hypothesized that E2f1 could repress the expression of upregulated genes through an HDAC (histone deacetylase)-dependent mechanism. To ascertain this hypothesis, Min6 cells were treated during $16 \mathrm{~h}$ with the pan-HDAC inhibitor trichostatin A (TSA, $0.5 \mu \mathrm{M}$ ). Interestingly, via RNA-seq, we evidenced that the expression of upregulated genes in $E 2 \mathrm{ft}^{\beta-1-}$ islets was significantly increased in response to TSA treatment compared to vehicle-treated cells whereas the expression of downregulated genes was not significantly modulated (Figure 5E), as observed in $E 2 f 1^{\beta-/-}$ islets. These results showed that the pharmacological HDAC inhibition in Min6 cells partly mimicked the effects observed in $E 2 f 1^{\beta-1-}$ pancreatic islets on the expression of upregulated genes, suggesting that an E2f1/HDAC complex could contribute to regulate the expression of these genes in the $\beta$ cell. Conversely, these results also indicated that E2f1-dependent downregulation of genes probably occurred through an HDAC-independent mechanism. To go further in the 
characterization of the molecular mechanisms linking E2f1 to HDAC in the $\beta$ cell, we measured the acetylation levels of promoter (TSS +/- $1 \mathrm{~kb}$ ) of up- and down-regulated genes in Min6 cells by probing H3K27ac level in response to TSA treatment through ChIPseq experiments. Interestingly, TSA treatment increased H3K27ac levels within the promoter of upregulated genes compared to vehicle-treated cells, whereas H3K27ac levels were less affected within the promoter of down-regulated genes (Figure 5F). In addition, H3K27me3 ChIP-seq signals were lower in the promoter region of upregulated genes upon TSA treatment (Figure 5G), whereas no effects were observed upon TSA treatment on downregulated genes. These results were in accordance with gene expression analysis (Figure 5E) and support a role for HDAC activity in the transcriptional repression of E2f1 target genes in $\beta$ cell whereas HDAC enzymes may probably not be involved in the E2f1-mediated transcriptional activation.

Interactomic analysis by rapid immunoprecipation mass spectometry of endogenous protein (RIME) identifies HDAC1 and HDAC6 as partners of E2f1 in $\beta$ cell.

We next asked whether E2f1 could directly interact with HDAC enzymes in $\beta$ cell. Rather than addressing this using coimmunoprecipitation strategies targeting a series of specific HDAC, we decided to follow a global interactomic approach by using the rapid immunoprecipitation mass spectrometry of endogenous protein (RIME) technology (Mohammed et al, 2013) enabling the identification of E2f1-associated proteins at proteome-wide level in Min6 cells. Consequently, E2f1 was over-expressed in Min6 cells through transfection of a plasmid encoding E2f1 cDNA fused to Flag tag followed by an anti-Flag-based E2f1 immunoprecipitation in order to identify E2f1 interacting proteins through mass spectrometry analysis. RIME analysis revealed that 245 peptides were found to be associated with E2f1 in Min6 cells (Table 2 and Supplementary figure 5A). As expected, E2f1 peptides were the most significantly enriched peptides thus validating our approach. Interestingly, peptides from HDAC6 and HDAC1 were also significantly enriched providing evidence that these two HDAC enzymes were associated with E2f1 in Min6 cells at the chromatin level (Table 2). Altogether, these results suggest that E2f1-mediated transcriptional repression may occur through interactions with HDAC1 and/or HDAC6. 


\section{Discussion}

In the present study, we show that the transcription factor E2F1 plays an essential role in maintaining $\beta$-cell function and identity through the control of transcriptomic and epigenetic programs within the pancreatic islets. Germ-line as well as $\beta$-cell specific E2f1 deficient mice exhibit impaired glucose homeostasis and decreased $\beta$-cell functions that are associated to a gene expression reprogramming. A detailed analysis of the transcriptome and epigenome of the pancreatic islet reveals that E2f1 is necessary to maintain several key genes for cell adhesion and inflammatory processes in a repressive state through the maintenance of epigenomic marks including H3K27me3 (Figure 5). We showed that the $\beta$ cell specific loss of E2f1 function also leads to an altered $\alpha$-to- $\beta$-cell ratio, while maintaining islet size, associated to insulin secretion defects in response to glucose and affects $\beta$-cell identity maintenance. In addition, through the use of the E2Fs inhibitor HLM006474, which selectively disrupts the binding of E2Fs to its target genes (Figure 4 and (Ma et al., 2008; Rosales-Hurtado et al., 2019)), we demonstrated here that a pharmacological intervention lowering the E2F1 transcriptional activity could mimic the genetic effects of E2f1 deficiency on the maintenance of $\beta$-cell identity and function, in mouse $\beta$-cell lines and human islets. Indeed, the treatment of human islets with this compound not only blunts $\beta$-cell function by impairing GSIS, but also leads to an alternative transcriptional program that affects the expression of $\alpha$ - and $\beta$-cell markers. Our results, associated to those showing that E2F1 overexpression stimulates $\beta$-cell proliferation and function (Grouwels et al, 2010), suggest that carefully targeting this pathway might be of interest for the maintenance of $\beta$-cell functions in the context of diabetes.

Our study is the first one to demonstrate a direct, cell-autonomous contribution of E2f1 in controlling not only GSIS but also $\beta$-cell identity maintenance, without affecting the pancreatic islet cell number. The role of E2F1 in the control of cell cycle and proliferation has been extensively studied, particularly in the context of cancer (Poppy Roworth et al., 2015). However, its function in non-proliferating fully differentiated cells, including $\beta$ cells, remains to be precisely deciphered. The observation that cell cycle regulators, including 
E2F1, are expressed in cells that are not proliferating, suggest that they may be involved in adaptive pathways that are independent of cellular proliferation. We and others have demonstrated that this pathway plays a key role in post-natal $\beta$-cell proliferation (Fajas et al, 2004; Iglesias et al, 2004; Li et al, 2003), glucose homeostasis and insulin secretion (Annicotte et al., 2009; Boni-Schnetzler et al, 2018; Grouwels et al., 2010). Studies also revealed that the Cdk4-E2F1-pRb pathway controls the fate of pancreatic progenitors through the transcriptional control of the expression of $\mathrm{Ngn} 3$ and $P d x 1$ as well as $\mathrm{Pdx} 1$ protein stability (Cai et al, 2013; Kim \& Rane, 2011; Kim et al, 2011). Most of these studies, including those from our group, were performed using germ-line E2f1 deficient mice, which precludes to ascertain a cell-autonomous role of E2f1 in the endocrine pancreas and $\beta$-cell functions. To better appreciate the specific role of E2F1 in $\beta$ cells, we specifically knocked-down E2f1 in the $\beta$ cell using the Cre/loxP technology. Although we cannot rule out an early role for E2f1 in pancreatic progenitors due to the use of the RIPCre mice (Herrera, 2000), our data demonstrate that E2f1 expression within the $\beta$ cell is necessary to maintain a proper gene expression to regulate insulin secretion and glucose homeostasis.

The complexity of E2F1 biology resides in the fact that it can positively or negatively regulate the expression of its target genes. Our data suggest that E2F1 is part of a repressor complex that regulates $\beta$ cell identity. In line with this, we observe that most of the genes with altered expression in $E 2 f 1^{\beta-/}$ isolated islets were upregulated, suggesting that the repressive effects of E2F1 are key in maintaining $\beta$-cell identity and subsequent $\beta$ cell function. Accordingly, repression of non $\beta$-cell programs is crucial for maintaining $\beta$ cell identity. Pdx1 (Gao et al., 2014), Pax6 (Swisa et al., 2017), Nkx6.1 (Schaffer et al., 2013) and Nkx2.2 (Gutierrez et al., 2017) are key transcription factor required to maintain gene repression of non- $\beta$-cell programs. Interestingly, Nkx6.1 mRNA levels are decreased in $E 2 f 1^{\beta-1-}$ isolated islets, suggesting that E2F1 could modulate $\beta$ cell functions through Nkx6.1 regulation. Chromatin regulators and epigenomic features play important roles in the control of $\beta$-cell identity and plasticity (Arda et al, 2016; Avrahami et al, 2015; Campbell \& Hoffman, 2016). Indeed, the modulation of islet enriched transcription factor activity, including Pdx1 or Nkx2.2, involves their interaction with coregulators such as Dnmt1, Dmnt3a or Hdac1 (Dhawan et al, 2011; Papizan et al, 2011; Spaeth et al, 2016) or their accessibility to chromatin, as demonstrated for the $\beta$-cell specific deletion of protein arginine methyltransferase 1 (Prmt1) that results in the loss of $\beta$-cell identity and diabetes development (Kim et al, 2020). In addition, recent studies also indicate that loss of 
Polycomb silencing in human and mouse $\beta$-cells contributes to loss of $\beta$-cell identity in diabetes (Lu et al., 2018). Indeed, the $\beta$-cell specific deletion of Eed, a component of the Polycomb repressive complex (PRC)-2, triggers $\beta$-cell dysfunctions, dedifferentiation and diabetes development associated to chromatin-state-associated transcriptional dysregulation (Lu et al., 2018). The findings that loss of PRC2 activity induces $\beta$-cell plasticity through epigenomic reprogramming at both active and silent genes suggest that maintaining proper and specific histone marks and chromatin state at precise loci is crucial to maintain normal $\beta$-cell functions and avoid T2D development. Interestingly, most of the upregulated genes in $E 2 f 1^{\beta-/}$ pancreatic islets were characterized by bivalent H3K4me3/H3K27me3 and Polycomb-repressed (H3K27me3) marks. In addition, epigenomic interventions triggering ectopic acetylation and gene derepression contribute to $\beta$-cell dysfunctions. Indeed, blocking HDAC activity through the use of the HDAC inhibitor SAHA, impairs glucose intolerance in mice fed a HFD (Lu et al., 2018). Here we show that the loss of E2f1 function triggers transcriptional dysregulation of specific genes that are in a bivalent (i.e. H3K4me3/H3K27me3 and Polycomb-repressed (H3K27me3) marks) and active (i.e. RNA-Pol2 recruitment, H3K4me3 and H3K27ac histone marks) state in healthy pancreatic islets, suggesting an ectopic acetylation in the promoter region of upregulated genes in $E 2 \mathrm{ft}^{\beta-/-}$ islets and decreased activation of the promoter region of downregulated genes. Accordingly, the use of the HDACi TSA also impairs the expression of genes regulated by E2f1 in pancreatic islets. Although E2F1 is a ubiquitous transcription factor that is weakly expressed within $\beta$ cell compared to bona fide $\beta$-cell genes, we speculate that this transcriptional regulator may cooperate with $\beta$-cell transcription factors and/or the chromatin machinery such as HDAC or the PRC2 complex to integrate some signals necessary for $\beta$-cell maintenance in physiological conditions. Since E2F1 regulates gene expression through its interaction with repressor complexes including $\mathrm{pRb}, \mathrm{SWI}$ SNF and HDACs (Brehm et al, 1998; Luo et al, 1998; Magnaghi-Jaulin et al, 1998), our finding that E2F1 mediates repression of non $\beta$-cell programs deserves deeper investigations to identify the E2F1 complexes that can trigger these transcriptomic and epigenomic effects in the $\beta$-cell and their physio(patho)logical consequences.

In summary, the present data highlight that E2F1 transcriptional activity within pancreatic islets is key for maintaining glucose homeostasis and insulin secretion through the regulation of key $\beta$-cell identity genes and the repression of non $\beta$-cell programs, both in mouse and human islets. The observation that E2F1 levels are decreased in human T2D 
islets (Lupi et al., 2008) suggests that a reduced E2F1 expression or activity may contribute to $\beta$-cell failure in diabetes.

Author contributions. F.O., C.B., X.G., M.M., C.C., E.C., N.R., L.R. and S.A.H. contributed to the in vivo and cellular experiments. E.D., S.A., L.B., M.D. and A.B. performed the RNA-seq and ChIP-seq experiments and analysis. P.D.D., Z.B., P.M., L.F., J.K.C. and F.P. provided reagents and data. P.F. discussed the results from the study. J.S.A. designed the study, supervised the project and contributed to experiments and/or their analysis and the funding of this project. F.O., C.B., N.R., P.F., A.B. and J.-S.A. wrote and/or edited the manuscript.

Acknowledgements. We thank Dr Patrick Collombat and Dr Raphael Scharfmann and members of the INSERM U1283/CNRS UMR 8199 for helpful discussions, and Céline Gheeraert for excellent help with ChIP experiments. Human islets were provided through the JDRF award 31-2008-416 (ECIT Islet for Basic Research program). The authors thank the Experimental Resources platform from Université de Lille, especially Cyrille Degraeve, Yann Lepage, Mélanie Besegher and Julien Devassine for animal care. We thank the Department of Histology from the Lille Medicine Faculty, particularly M.H. Gevaert and R.M. Siminski, for histological preparations. This work was supported by grants from «European Genomic Institute for Diabetes » E.G.I.D, ANR-10-LABX-46 and Equipex 2010 ANR-10-EQPX-07-01; 'LIGAN-PM' Genomics platform, a French State fund managed by the Agence Nationale de la Recherche under the frame program Investissements d'Avenir I-SITE ULNE / ANR-16-IDEX-0004 ULNE (to P.F., A.B. and J-S. A), Agence Nationale pour la Recherche (BETAPLASTICITY, ANR-17-CE14-0034 to J-S. A.), European Foundation for the Study of Diabetes (EFSD, to J-S.A.), European Commission, European Research Council (GEPIDIAB 294785 to P.F.), INSERM, CNRS, Institut Pasteur de Lille, Association pour la Recherche sur le Diabète (to J-S.A.), Université de Lille (to F.O., C.B., X.G., N.R. and J-S.A.), I-SITE ULNE (EpiRNAdiab Sustain grant to J-S.A.), Conseil Régional Hauts de France and Métropole Européenne de Lille (to X.G., N.R. and J-S.A.), F.E.D.E.R. (Fonds Européen de Développement Régional, to N.R., P.F. and JS.A.) and Société Francophone du Diabète (to S.A.H. and J-S.A).

Conflict of interest. The authors declare no competing financial interests. 


\section{Figure legends}

Figure 1. Altered $\alpha$-to- $\beta$ cell ratio in germline E2f1-deficient mice. (A) Representative immunofluorescent staining of insulin and glucagon in pancreatic sections from 16 week old global E2f1 knockout male mice $\left(E 2 f 1^{-1-}\right)$ compared to littermate controls $\left(E 2 f 1^{+/+}\right)$. (B) Quantification of glucagon (Glucagon + ) and insulin (Insulin + ) labelled cells from $(A, n=3$ per gentotype). (C) Representative immunofluorescent staining of insulin and glucagon in pancreatic sections from 14 to 15 week old global E2f1 knockout male mice in a $D b / D b$ background $\left(D b / D b:: E 2 f 1^{-/-}\right)$compared to littermate control $\left(D b / D b:: E 2 f 1^{+/+}\right)$. (D) Quantification of glucagon (Glucagon + ) and insulin (Insulin + ) labelled cells in $D b / D b:: E 2 f 1^{-/-}$and $D b / D b:: E 2 f 1^{+/+}$mice calculated from $C$ ( $\mathrm{n}=4$ per genotype). Values in $\mathrm{B}$ and $D$ are expressed as mean \pm s.e.m. and were analysed by two-tailed unpaired $t$-test. ${ }^{*} p<0.05 ;{ }^{* *} p<0.01$.

Figure 2. The $\beta$ cell-specific deletion of E2f1 impairs glucose tolerance and insulin secretion in mice. (A) Intraperitoneal glucose tolerance test (IPGTT) was performed on $E_{2 f 1^{\beta-/-}}(n=12)$, RIP-Cre ${ }^{/+}(n=7)$ and E2ff $f^{\text {Il/fl }}(n=8)$ male mice at 12 weeks of age. (B) Area under the curve (AUC) calculated from (B). (C) Plasma insulin levels at 0 and 30 min after intraperitoneal glucose injection in $E 2 f 1^{\beta-/-}(n=18), R_{P}-C r e^{/+}(n=15)$ and $E 2 f 1^{f l / f l}(n=6)$ male mice at 12 weeks of age. (D) Intraperitoneal insulin tolerance test (IPITT) of $E 2 f 1^{\beta--}(n=8)$, RIP-Cre $^{/+}(\mathrm{n}=8)$ and E2f1 $1^{\mathrm{fl} / \mathrm{fl}}(\mathrm{n}=8)$ male mice at 12 weeks of age. (E) Glucose-stimulated insulin secretion (GSIS) at indicated glucose concentrations on islets isolated from $E 2 f 1^{\beta-/-}$ $(\mathrm{n}=3)$ and control E2f1//fll $(\mathrm{n}=5)$ male mice. (F) Representative immunofluorescent staining of insulin and glucagon in pancreatic sections from 3 months old $E 2 f^{\beta-/-}$ and control $E 2 f 1^{\mathrm{fl} / \mathrm{fl}}$ male mice. (G) Ratio of glucagon labelled cells (Glucagon + ) over insulin labelled cells (Insulin + ) in $E 2 f 1^{\beta-/-}$ and $E 2 f 1^{f / / f l}$ male mice calculated from (G). All values are expressed as mean \pm s.e.m. and were analysed by one-way analysis of variance (ANOVA) with Tukey's test (A, C, D), two-way ANOVA with Tukey's test (B, E, F) or two-tailed unpaired $t$-test $(\mathrm{H}) .{ }^{*} \mathrm{p}<0.05 ;{ }^{* *} \mathrm{p}<0.01 ;{ }^{\star * *} \mathrm{p}<0.001$.

Figure 3. RNA-seq analysis of E2f1 $\beta$ cell-specific knockout islets reveal altered transcriptional programs. (A) Volcano plot providing adjusted $P$ value (i.e., FDR, false discovery rate) and fold change for all gene transcripts in islets from $E 2 f 1^{\beta-/-}$ mice. Genes that are differentially expressed compared to $E 2 f 1^{\mathrm{fl} / \mathrm{fl}}$ with an $\mathrm{Adj} \mathrm{P}<0.05$ are indicated by 
two-level color coding. 199 downregulated protein-coding genes are highlighted in red. 493 upregulated protein-coding genes are highlighted in green ( $n=3$ per genotype for RNA-seq analysis). (B-C) Metascape enrichment analysis of downregulated (B) and upregulated $(C)$ genes in $E 2 f 1^{\beta-/-}$ compared to $E 2 f 1^{f / / f l}$. Histogram of enriched terms across input gene lists are shown. (D) Evolutionary conserved $\alpha$ - and $\beta$-cell markers were recovered from Tarifeno-Saldivia $\mathrm{E}$ et al. (Tarifeno-Saldivia et al., 2017) and were used to filter our RNA-seq dataset. Volcano plots provides AdjP values (i.e., FDR, false discovery rate) and fold change for both $\alpha$ - and $\beta$-cell marker transcripts in islets from $E 2 f 1^{\beta-/-}$ mice. The $\alpha$ - and $\beta$-cell markers are displayed as blue triangle and red square, respectively. Gene symbols of differentially expressed $\alpha$ - and $\beta$-cell markers are displayed (AdjP<0.05). (E-F) Enrichment plot from Gene Set Enrichment Analysis (GSEA) was conducted with 82 probe sets specifically expressed in $\alpha$-cells $(E)$ and with 26 probe sets specifically expressed in $\beta$-cells $(F)$.

Figure 4. The pharmacological inhibition of E2f transcription factor activity induces a loss of $\beta$-cell function in mouse and human islets. (A) Min6 cells were transiently cotransfected with the E2F-RE-Tk promoter luciferase construct in the absence (PCDNA3) or presence of E2F1:DP1 heterodimer (E2F1/DP1) and were subsequently treated with DMSO $(0.1 \%, 48 \mathrm{~h})$ or HLM006474-treated (10 $\mu \mathrm{M}, 48 \mathrm{~h})$. Results were normalized to $\beta$ galactosidase activity. (B) Glucose stimulated insulin secretion (GSIS) at indicated glucose concentration on DMSO $(0.1 \%$, 48h) or HLM006474-treated (10 $\mu \mathrm{M}, 48 \mathrm{~h})$ Min6 cells $(\mathrm{n}=6)$. (C) qPCR-based analysis of $\beta$-cell specific mRNA expression in DMSO $(0.1 \%, 48 \mathrm{~h})$ or HLM006474-treated (10 $\mathrm{MM}$, 48h) Min6 cells ( $\mathrm{n=3}$ ). (D) Glucose stimulated insulin secretion (GSIS) at indicated glucose concentration on human islets treated with DMSO $(0.1 \%, 48 h)$ or HLM006474 (10 $\mu \mathrm{M}, 48 h)(n=3)$ (see supplementary Table S1 for donor information). (E) qPCR-based analysis of $\beta$-cell specific (INS, PDX1, PAX4, MAFA and NEUROD1) and $\alpha$-cell specific ( $A R X$ and GCG) mRNA expression in DMSO $(0.1 \%$, 48h) or HLM006474-treated (10 $\mu \mathrm{M}, 48 \mathrm{~h})$ human islets $(\mathrm{n}=3)$. Data are represented as mean \pm s.e.m. and were analyzed by two-way ANOVA with Tukey's test $(A, B, D)$ and two-tailed unpaired $t$-test $(C, E) .{ }^{*} p<0.05 ;{ }^{* *} p<0.01 ;{ }^{* * *} p<0.001$.

Figure 5. The transcriptional repression of E2f1 $\beta$-cell target genes occurs through an HDAC-dependent mechanism. (A) Expression level of $E 2 f 1^{\beta-/-}$ up- and downregulated genes both in pancreatic islets from control (WT) and E2f1 knock-out $\left(E 2 f 1^{\beta-I_{-}}\right)$ 
mice. Results are displayed as Log10(TPM+1) calculated from TPM obtained from RNAseq data. Data are represented as mean s.e.m and were analyzed by unpaired $t$-test ${ }^{* * * *}$ $\mathrm{p}<0.0001)$. (B) Pie-chart displaying chromatin state of $E 2 f 1^{\beta-/}$ up- and down- regulated genes according to pancreatic islets genes-associated chromatin state stratification from Lu TT et al. (Lu et al., 2018) (C) H3K4me3 and H3K27ac ChIP-seq mean signal within promoter (centered to TSS +/- $1 \mathrm{~kb}$ ) of $E 2 f 1^{\beta-/}$ up- and down- regulated genes both in Min6 cells and mouse pancreatic islets (Lu et al., 2018). (D) H3K27me3 ChIP-seq mean signal within promoter (centered to TSS +/- $1 \mathrm{~kb}$ ) of $E 2 \mathrm{ft}^{\beta-/-} \mathrm{up}$ - and down- regulated genes both in Min6 cells and mouse pancreatic islets (Lu et al., 2018). (E) Expression level of E2f1 ${ }^{\beta-\gamma_{-}}$upand down- regulated genes in TSA-treated Min6 cells. Results are displayed as Log10(TPM+1) calculated from TPM obtained from RNA-seq data. Data are represented as mean \pm s.e.m and were analyzed by unpaired $t$-test $\left(^{\star \star * *} p<0.0001\right)$. (F) H3K27ac ChIPseq mean signal within promoter (centered to TSS +/- $1 \mathrm{~kb}$ ) of $E 2 f 1^{\beta-/-}$ up- and downregulated genes in TSA-treated Min6 cells. (G) H3K27me3 ChIP-seq mean signal within promoter (centered to TSS +/- $1 \mathrm{~kb}$ ) of $E 2 \mathrm{ft}^{\beta-/}$ up- and down- regulated genes in TSAtreated Min6 cells.

Table 1. Pathway analysis of RNA-sequencing data in pancreatic islets isolated from control $E 2 f 1^{f / / f l}$ and $\beta$-cell specific $E 2 f 1^{\beta-/-}$ deficient mice identifies potential upstream regulators associated to E2f1 deficiency.

Table 2. List of peptides associated to E2F1 in Min6 cells identified through RIME experiments. RIME was performed on biological duplicates and common peptides found in both replicates are shown.

\section{References}

Annicotte JS, Blanchet E, Chavey C, lankova I, Costes S, Assou S, Teyssier J, Dalle S, Sardet C, Fajas L (2009) The CDK4-pRB-E2F1 pathway controls insulin secretion. Nat Cell Biol 11: 1017-1023

Arda HE, Li L, Tsai J, Torre EA, Rosli Y, Peiris H, Spitale RC, Dai C, Gu X, Qu K et al (2016) Age-Dependent Pancreatic Gene Regulation Reveals Mechanisms Governing Human beta Cell Function. Cell Metab 23: 909-920

Avrahami D, Li C, Zhang J, Schug J, Avrahami R, Rao S, Stadler MB, Burger L, Schubeler D, Glaser B et al (2015) Aging-Dependent Demethylation of Regulatory Elements Correlates with Chromatin State and Improved beta Cell Function. Cell Metab 22: 619-632 Avrahami D, Wang YJ, Schug J, Feleke E, Gao L, Liu C, Consortium H, Naji A, Glaser B, Kaestner KH (2020) Single-cell transcriptomics of human islet ontogeny defines the molecular basis of beta-cell dedifferentiation in T2D. Molecular metabolism 42: 101057 
Boni-Schnetzler M, Hauselmann SP, Dalmas E, Meier DT, Thienel C, Traub S, Schulze F, Steiger L, Dror E, Martin P et al (2018) beta Cell-Specific Deletion of the IL-1 Receptor Antagonist Impairs beta Cell Proliferation and Insulin Secretion. Cell Rep 22: 1774-1786 Bramswig NC, Everett LJ, Schug J, Dorrell C, Liu C, Luo Y, Streeter PR, Naji A, Grompe $\mathrm{M}$, Kaestner KH (2013) Epigenomic plasticity enables human pancreatic alpha to beta cell reprogramming. J Clin Invest 123: 1275-1284

Brehm A, Miska EA, McCance DJ, Reid JL, Bannister AJ, Kouzarides T (1998) Retinoblastoma protein recruits histone deacetylase to repress transcription. Nature 391: 597-601.

Brereton MF, Iberl M, Shimomura K, Zhang Q, Adriaenssens AE, Proks P, Spiliotis, II, Dace W, Mattis KK, Ramracheya R et al (2014) Reversible changes in pancreatic islet structure and function produced by elevated blood glucose. Nat Commun 5: 4639

Cai EP, Wu X, Schroer SA, Elia AJ, Nostro MC, Zacksenhaus E, Woo M (2013) Retinoblastoma tumor suppressor protein in pancreatic progenitors controls alpha- and beta-cell fate. Proc Natl Acad Sci U S A 110: 14723-14728

Campbell SA, Hoffman BG (2016) Chromatin Regulators in Pancreas Development and Diabetes. Trends Endocrinol Metab 27: 142-152

Chakravarthy $\mathrm{H}$, Gu X, Enge M, Dai X, Wang Y, Damond N, Downie C, Liu K, Wang J, Xing $Y$ et al (2017) Converting Adult Pancreatic Islet alpha Cells into beta Cells by Targeting Both Dnmt1 and Arx. Cell Metab 25: 622-634

Collombat P, Xu X, Ravassard P, Sosa-Pineda B, Dussaud S, Billestrup N, Madsen OD, Serup P, Heimberg H, Mansouri A (2009) The ectopic expression of Pax4 in the mouse pancreas converts progenitor cells into alpha and subsequently beta cells. Cell 138: 449462

Denechaud PD, Fajas L, Giralt A (2017) E2F1, a Novel Regulator of Metabolism. Frontiers in endocrinology 8: 311

Denechaud PD, Lopez-Mejia IC, Giralt A, Lai Q, Blanchet E, Delacuisine B, Nicolay BN, Dyson NJ, Bonner C, Pattou F et al (2016) E2F1 mediates sustained lipogenesis and contributes to hepatic steatosis. J Clin Invest 126: 137-150

Dhawan S, Georgia S, Tschen SI, Fan G, Bhushan A (2011) Pancreatic beta cell identity is maintained by DNA methylation-mediated repression of Arx. Dev Cell 20: 419-429

Dor Y, Glaser B (2013) beta-cell dedifferentiation and type 2 diabetes. N Engl J Med 368: 572-573

Ediger BN, Lim HW, Juliana C, Groff DN, Williams LT, Dominguez G, Liu JH, Taylor BL, Walp ER, Kameswaran V et al (2017) LIM domain-binding 1 maintains the terminally differentiated state of pancreatic beta cells. J Clin Invest 127: 215-229

Fajas L, Annicotte JS, Miard S, Sarruf D, Watanabe M, Auwerx J (2004) Impaired pancreatic growth, beta cell mass, and beta cell function in E2F1 (-/- )mice. J Clin Invest 113: $1288-1295$

Gao T, McKenna B, Li C, Reichert M, Nguyen J, Singh T, Yang C, Pannikar A, Doliba N, Zhang T et al (2014) Pdx1 maintains beta cell identity and function by repressing an alpha cell program. Cell Metab 19: 259-271

Giralt A, Denechaud PD, Lopez-Mejia IC, Delacuisine B, Blanchet E, Bonner C, Pattou F, Annicotte JS, Fajas L (2018) E2F1 promotes hepatic gluconeogenesis and contributes to hyperglycemia during diabetes. Molecular metabolism 11: 104-112

Grouwels G, Cai Y, Hoebeke I, Leuckx G, Heremans Y, Ziebold U, Stange G, Chintinne M, Ling Z, Pipeleers D et al (2010) Ectopic expression of E2F1 stimulates beta-cell proliferation and function. Diabetes 59: 1435-1444

Gutierrez GD, Bender AS, Cirulli V, Mastracci TL, Kelly SM, Tsirigos A, Kaestner KH, Sussel $L$ (2017) Pancreatic beta cell identity requires continual repression of non-beta cell programs. J Clin Invest 127: 244-259 
Helman A, Klochendler A, Azazmeh N, Gabai Y, Horwitz E, Anzi S, Swisa A, Condiotti R, Granit RZ, Nevo Y et al (2016) p16(Ink4a)-induced senescence of pancreatic beta cells enhances insulin secretion. Nat Med 22: 412-420

Herrera PL (2000) Adult insulin- and glucagon-producing cells differentiate from two independent cell lineages. Development 127: 2317-2322

Iglesias A, Murga M, Laresgoiti U, Skoudy A, Bernales I, Fullaondo A, Moreno B, Lloreta J, Field SJ, Real FX et al (2004) Diabetes and exocrine pancreatic insufficiency in E2F1/E2F2 double-mutant mice. J Clin Invest 113: 1398-1407

Kent LN, Leone G (2019) The broken cycle: E2F dysfunction in cancer. Nat Rev Cancer 19: $326-338$

Kerr-Conte J, Vandewalle B, Moerman E, Lukowiak B, Gmyr V, Arnalsteen L, Caiazzo R, Sterkers A, Hubert T, Vantyghem MC et al (2010) Upgrading pretransplant human islet culture technology requires human serum combined with media renewal. Transplantation 89: 1154-1160

Kim H, Yoon BH, Oh CM, Lee J, Lee K, Song H, Kim E, Yi K, Kim MY, Kim H et al (2020) PRMT1 Is Required for the Maintenance of Mature beta-Cell Identity. Diabetes 69: 355368

Kim SY, Rane SG (2011) The Cdk4-E2f1 pathway regulates early pancreas development by targeting Pdx1+ progenitors and Ngn3+ endocrine precursors. Development 138: 19031912

Kim YC, Kim SY, Mellado-Gil JM, Yadav H, Neidermyer W, Kamaraju AK, Rane SG (2011) RB regulates pancreas development by stabilizing Pdx1. EMBO J 30: 1563-1576

Lawlor N, Stitzel ML (2019) (Epi)genomic heterogeneity of pancreatic islet function and failure in type 2 diabetes. Molecular metabolism 27S: S15-S24

Li FX, Zhu JW, Tessem JS, Beilke J, Varella-Garcia M, Jensen J, Hogan CJ, DeGregori J (2003) The development of diabetes in E2f1/E2f2 mutant mice reveals important roles for bone marrow-derived cells in preventing islet cell loss. Proc Natl Acad Sci U S A 100: $12935-12940$

Lu TT, Heyne S, Dror E, Casas E, Leonhardt L, Boenke T, Yang CH, Sagar, Arrigoni L, Dalgaard K et al (2018) The Polycomb-Dependent Epigenome Controls beta Cell Dysfunction, Dedifferentiation, and Diabetes. Cell Metab 27: 1294-1308 e1297

Luo RX, Postigo AA, Dean DC (1998) Rb interacts with histone deacetylase to repress transcription. Cell 92: 463-473.

Lupi R, Mancarella R, Del Guerra S, Bugliani M, Del Prato S, Boggi U, Mosca F, Filipponi F, Marchetti P (2008) Effects of exendin-4 on islets from type 2 diabetes patients. Diabetes Obes Metab 10: 515-519

Ma Y, Kurtyka CA, Boyapalle S, Sung SS, Lawrence H, Guida W, Cress WD (2008) A small-molecule E2F inhibitor blocks growth in a melanoma culture model. Cancer Res 68: 6292-6299

Magnaghi-Jaulin L, Groisman R, Naguibneva I, Robin P, Lorain S, Le Villain JP, Troalen F, Trouche D, Harel-Bellan A (1998) Retinoblastoma protein represses transcription by recruiting a histone deacetylase. Nature 391: 601-605.

Martin J, Hunt SL, Dubus P, Sotillo R, Nehme-Pelluard F, Magnuson MA, Parlow AF, Malumbres M, Ortega S, Barbacid M (2003) Genetic rescue of Cdk4 null mice restores pancreatic beta-cell proliferation but not homeostatic cell number. Oncogene 22: 52615269

Miyawaki K, Inoue H, Keshavarz P, Mizuta K, Sato A, Sakamoto Y, Moritani M, Kunika K, Tanahashi T, Itakura M (2008) Transgenic expression of a mutated cyclin-dependent kinase 4 (CDK4/R24C) in pancreatic beta-cells prevents progression of diabetes in $\mathrm{db} / \mathrm{db}$ mice. Diabetes research and clinical practice 82: 33-41 
Mohammed H, D'Santos C, Serandour AA, Ali HR, Brown GD, Atkins A, Rueda OM, Holmes KA, Theodorou V, Robinson JL et al (2013) Endogenous purification reveals GREB1 as a key estrogen receptor regulatory factor. Cell Rep 3: 342-349

Ndiaye FK, Ortalli A, Canouil M, Huyvaert M, Salazar-Cardozo C, Lecoeur C, Verbanck M, Pawlowski V, Boutry R, Durand E et al (2017) Expression and functional assessment of candidate type 2 diabetes susceptibility genes identify four new genes contributing to human insulin secretion. Molecular metabolism 6: 459-470

Nicol JW, Helt GA, Blanchard SG, Jr., Raja A, Loraine AE (2009) The Integrated Genome Browser: free software for distribution and exploration of genome-scale datasets. Bioinformatics 25: 2730-2731

Pal A, Potjer TP, Thomsen SK, Ng HJ, Barrett A, Scharfmann R, James TJ, Bishop DT, Karpe F, Godsland IF et al (2016) Loss-of-Function Mutations in the Cell-Cycle Control Gene CDKN2A Impact on Glucose Homeostasis in Humans. Diabetes 65: 527-533

Papizan JB, Singer RA, Tschen SI, Dhawan S, Friel JM, Hipkens SB, Magnuson MA, Bhushan A, Sussel L (2011) Nkx2.2 repressor complex regulates islet beta-cell specification and prevents beta-to-alpha-cell reprogramming. Genes Dev 25: 2291-2305

Poppy Roworth A, Ghari F, La Thangue NB (2015) To live or let die - complexity within the E2F1 pathway. Mol Cell Oncol 2: e970480

Rabhi N, Denechaud PD, Gromada X, Hannou SA, Zhang H, Rashid T, Salas E, Durand $\mathrm{E}$, Sand O, Bonnefond A et al (2016) KAT2B is required for pancreatic beta cell adaptation to metabolic stress by controlling the unfolded protein response signaling. Cell Reports In press

Rai V, Quang DX, Erdos MR, Cusanovich DA, Daza RM, Narisu N, Zou LS, Didion JP, Guan Y, Shendure J et al (2020) Single-cell ATAC-Seq in human pancreatic islets and deep learning upscaling of rare cells reveals cell-specific type 2 diabetes regulatory signatures. Molecular metabolism 32: 109-121

Rane SG, Dubus P, Mettus RV, Galbreath EJ, Boden G, Reddy EP, Barbacid M (1999) Loss of Cdk4 expression causes insulin-deficient diabetes and Cdk4 activation results in beta-islet cell hyperplasia. Nat Genet 22: 44-52

Rosales-Hurtado M, Lebeau A, Bourouh C, Cebrian-Torrejon G, Albalat M, Jean M, Naubron JV, Annicotte JS, Benfodda Z, Meffre P (2019) Improved synthesis, resolution, absolute configuration determination and biological evaluation of HLM006474 enantiomers. Bioorg Med Chem Lett 29: 380-382

Salas E, Rabhi N, Froguel P, Annicotte JS (2014) Role of Ink4a/Arf locus in beta cell mass expansion under physiological and pathological conditions. J Diabetes Res 2014: 873679

Sangwan M, McCurdy SR, Livne-Bar I, Ahmad M, Wrana JL, Chen D, Bremner R (2012) Established and new mouse models reveal E2f1 and Cdk2 dependency of retinoblastoma, and expose effective strategies to block tumor initiation. Oncogene 31: 5019-5028

Schaffer AE, Taylor BL, Benthuysen JR, Liu J, Thorel F, Yuan W, Jiao Y, Kaestner KH, Herrera PL, Magnuson MA et al (2013) Nkx6.1 controls a gene regulatory network required for establishing and maintaining pancreatic Beta cell identity. PLoS Genet 9: e1003274

Segerstolpe A, Palasantza A, Eliasson P, Andersson EM, Andreasson AC, Sun X, Picelli S, Sabirsh A, Clausen M, Bjursell MK et al (2016) Single-Cell Transcriptome Profiling of Human Pancreatic Islets in Health and Type 2 Diabetes. Cell Metab 24: 593-607

Serrano M, Lee H, Chin L, Cordon-Cardo C, Beach D, DePinho RA (1996) Role of the INK4a locus in tumor suppression and cell mortality. Cell 85: 27-37

Sotillo R, Dubus P, Martin J, de la Cueva E, Ortega S, Malumbres M, Barbacid M (2001) Wide spectrum of tumors in knock-in mice carrying a Cdk4 protein insensitive to INK4 inhibitors. EMBO J 20: 6637-6647

Spaeth JM, Walker EM, Stein R (2016) Impact of Pdx1-associated chromatin modifiers on islet beta-cells. Diabetes Obes Metab 18 Suppl 1: 123-127 
Spijker HS, Ravelli RB, Mommaas-Kienhuis AM, van Apeldoorn AA, Engelse MA, Zaldumbide A, Bonner-Weir S, Rabelink TJ, Hoeben RC, Clevers H et al (2013) Conversion of mature human beta-cells into glucagon-producing alpha-cells. Diabetes 62: 2471-2480

Spijker HS, Song H, Ellenbroek JH, Roefs MM, Engelse MA, Bos E, Koster AJ, Rabelink TJ, Hansen BC, Clark A et al (2015) Loss of beta-Cell Identity Occurs in Type 2 Diabetes and Is Associated With Islet Amyloid Deposits. Diabetes 64: 2928-2938

Subramanian A, Tamayo P, Mootha VK, Mukherjee S, Ebert BL, Gillette MA, Paulovich A, Pomeroy SL, Golub TR, Lander ES et al (2005) Gene set enrichment analysis: a knowledge-based approach for interpreting genome-wide expression profiles. Proc Natl Acad Sci U S A 102: 15545-15550

Swisa A, Avrahami D, Eden N, Zhang J, Feleke E, Dahan T, Cohen-Tayar Y, StolovichRain M, Kaestner KH, Glaser B et al (2017) PAX6 maintains beta cell identity by repressing genes of alternative islet cell types. J Clin Invest 127: 230-243

Talchai C, Xuan S, Lin HV, Sussel L, Accili D (2012) Pancreatic $\beta$ cell dedifferentiation as a mechanism of diabetic $\beta$ cell failure. Cell 150: 1223-1234

Tarifeno-Saldivia E, Lavergne A, Bernard A, Padamata K, Bergemann D, Voz ML, Manfroid I, Peers B (2017) Transcriptome analysis of pancreatic cells across distant species highlights novel important regulator genes. BMC Biol 15: 21

Thorel F, Nepote V, Avril I, Kohno K, Desgraz R, Chera S, Herrera PL (2010) Conversion of adult pancreatic alpha-cells to beta-cells after extreme beta-cell loss. Nature 464: 11491154

Wang P, Fiaschi-Taesch NM, Vasavada RC, Scott DK, Garcia-Ocana A, Stewart AF (2015) Diabetes mellitus--advances and challenges in human beta-cell proliferation. Nat Rev Endocrinol 11: 201-212

Wang YJ, Schug J, Won KJ, Liu C, Naji A, Avrahami D, Golson ML, Kaestner KH (2016) Single-Cell Transcriptomics of the Human Endocrine Pancreas. Diabetes 65: 3028-3038

Weir GC, Bonner-Weir S (2004) Five stages of evolving beta-cell dysfunction during progression to diabetes. Diabetes 53 Suppl 3: S16-21

White MG, Marshall HL, Rigby R, Huang GC, Amer A, Booth T, White S, Shaw JA (2013) Expression of mesenchymal and alpha-cell phenotypic markers in islet beta-cells in recently diagnosed diabetes. Diabetes Care 36: 3818-3820

Xin Y, Kim J, Okamoto H, Ni M, Wei Y, Adler C, Murphy AJ, Yancopoulos GD, Lin C, Gromada J (2016) RNA Sequencing of Single Human Islet Cells Reveals Type 2 Diabetes Genes. Cell Metab 24: 608-615

Yang Y-p, Thorel F, Boyer DF, Herrera PL, Wright CVE (2011) Context-specific a -to- $\beta$ cell reprogramming by forced $\mathrm{Pdx} 1$ expression service re programming by forced $\mathrm{Pdx} 1$ expression. 1680-1685

Zhou Y, Zhou B, Pache L, Chang M, Khodabakhshi AH, Tanaseichuk O, Benner C, Chanda SK (2019) Metascape provides a biologist-oriented resource for the analysis of systems-level datasets. Nat Commun 10: 1523 
A

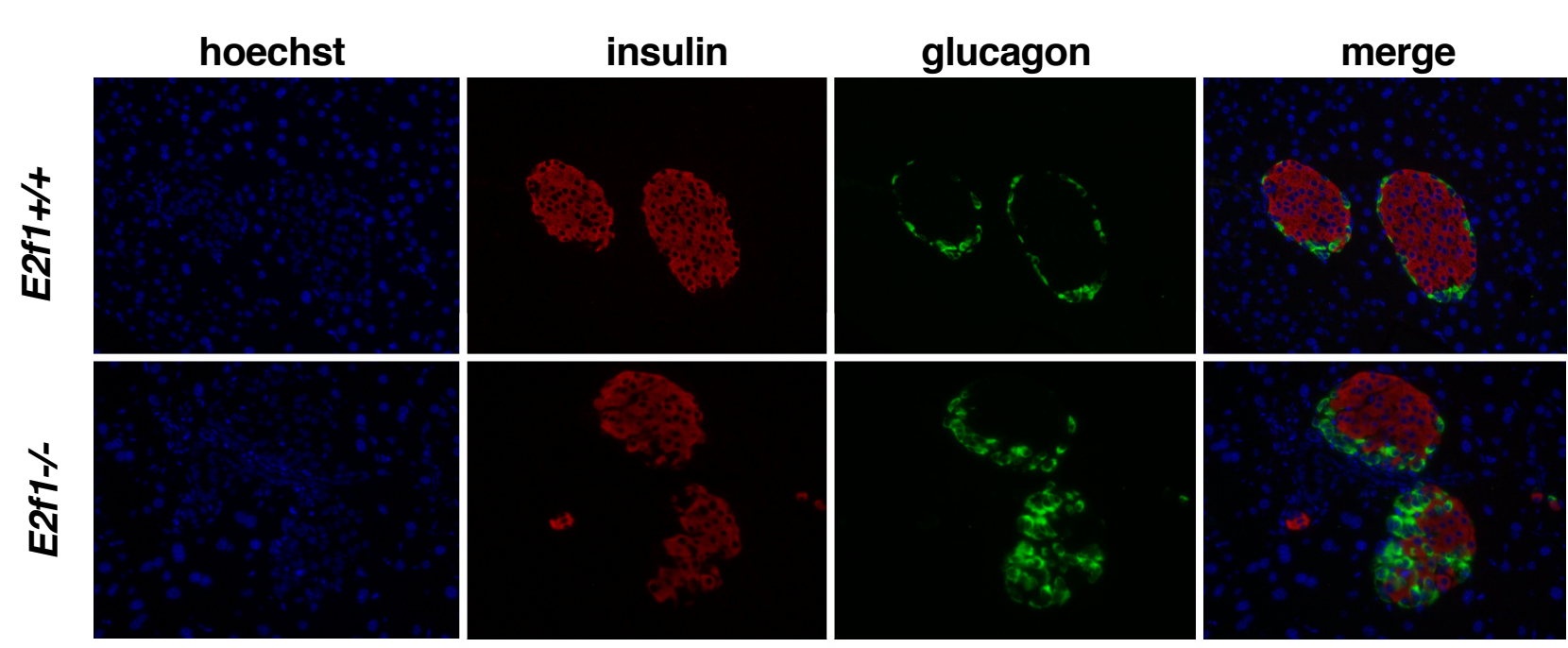

C

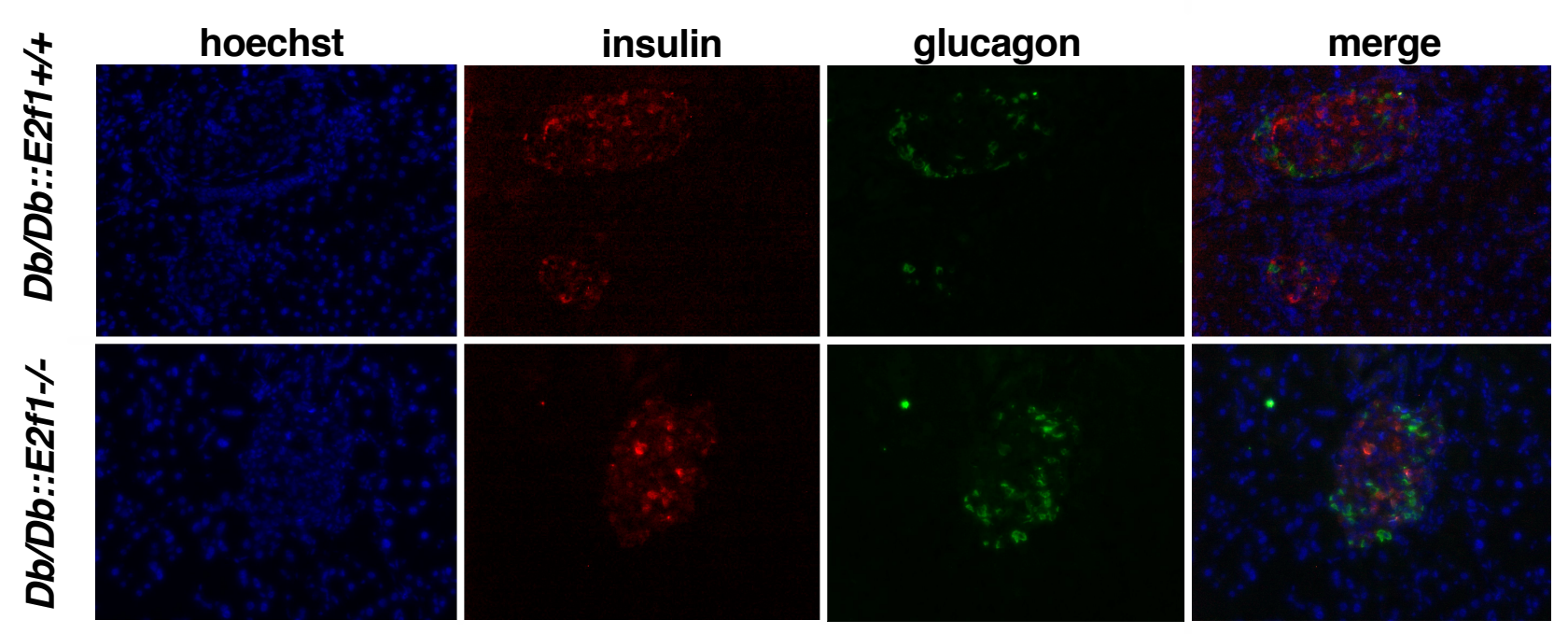

B

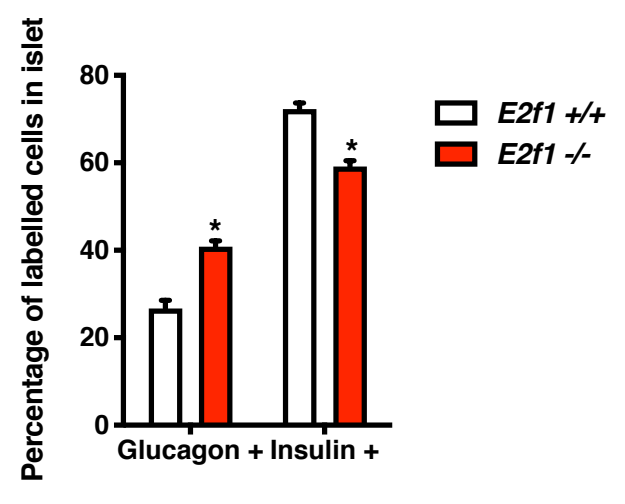

D

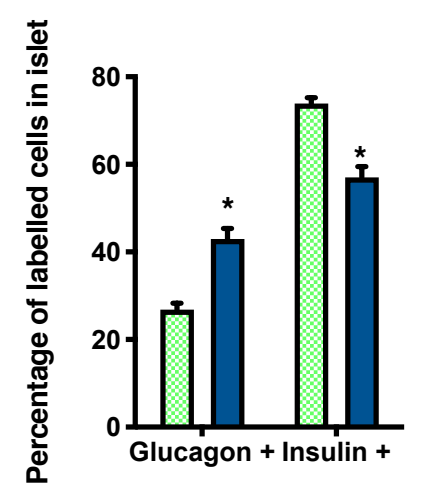

$\square D b / D b:: E 2 f 1+/+$

口 Db/Db::E2f1 - 
A

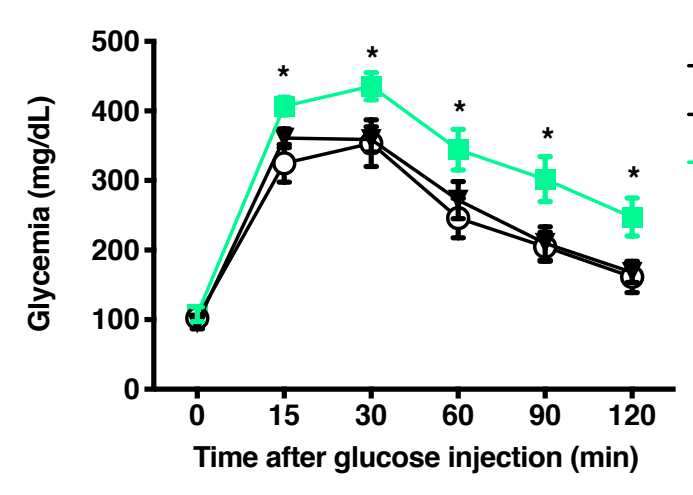

B

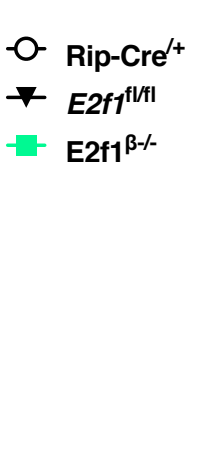

C

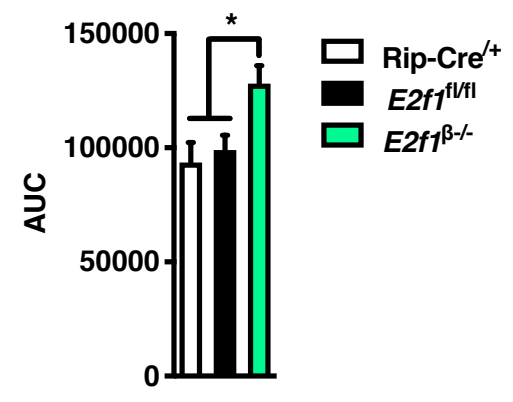

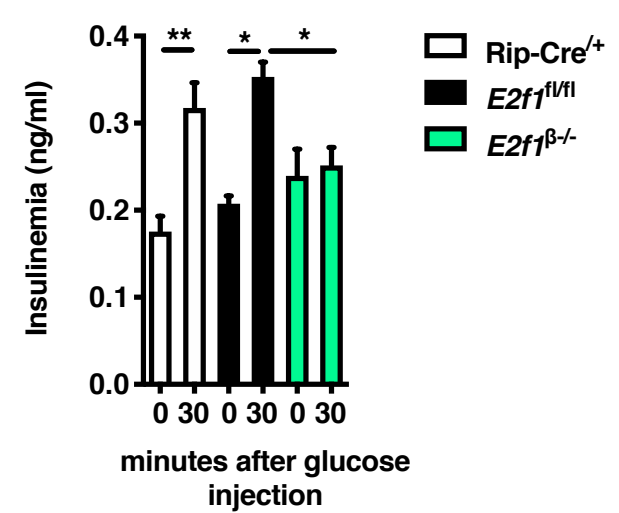

D

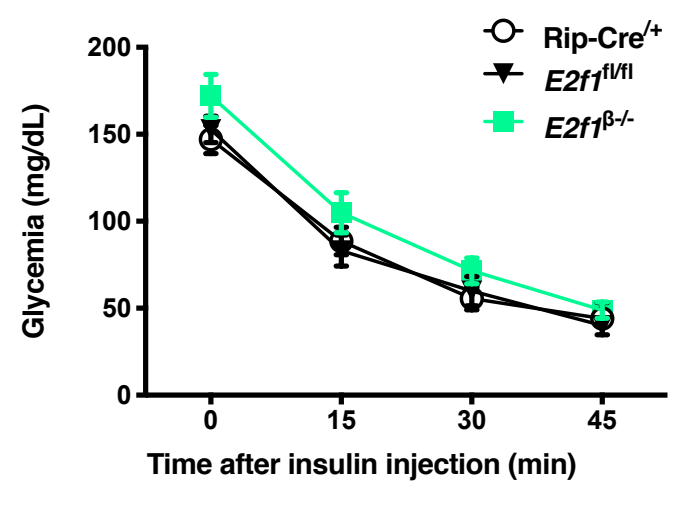

E

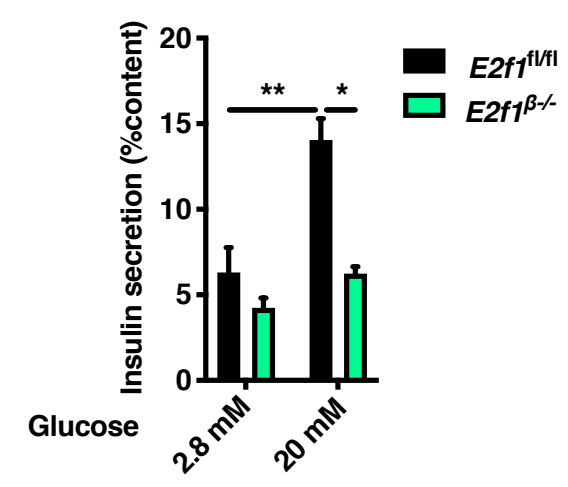

F

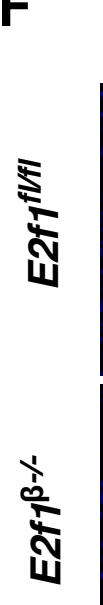

insulin

glucagon
G

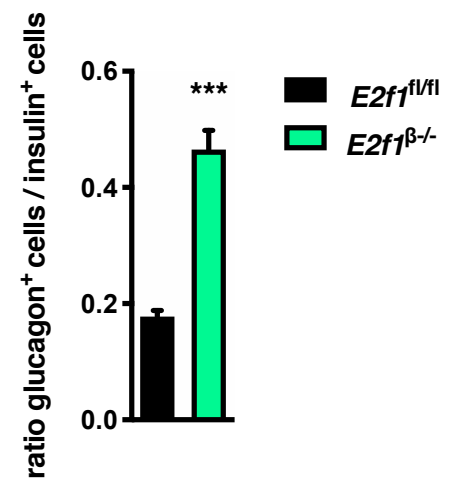



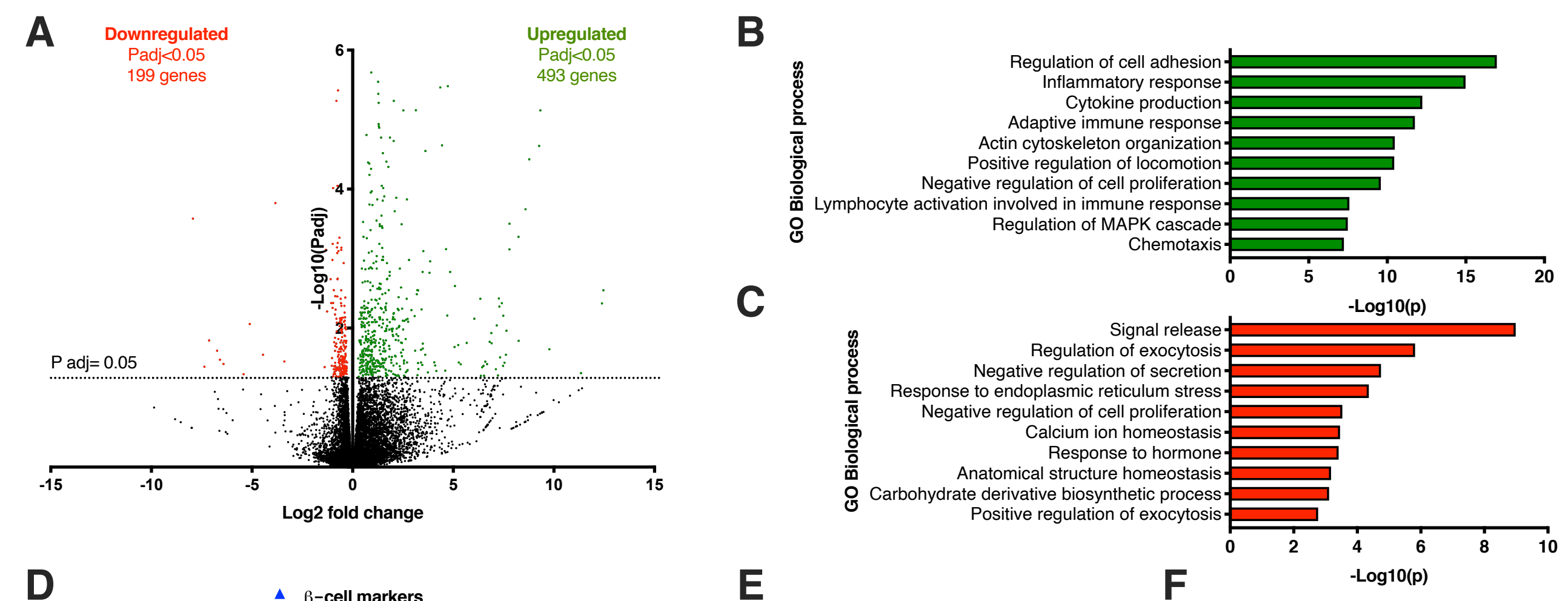

D

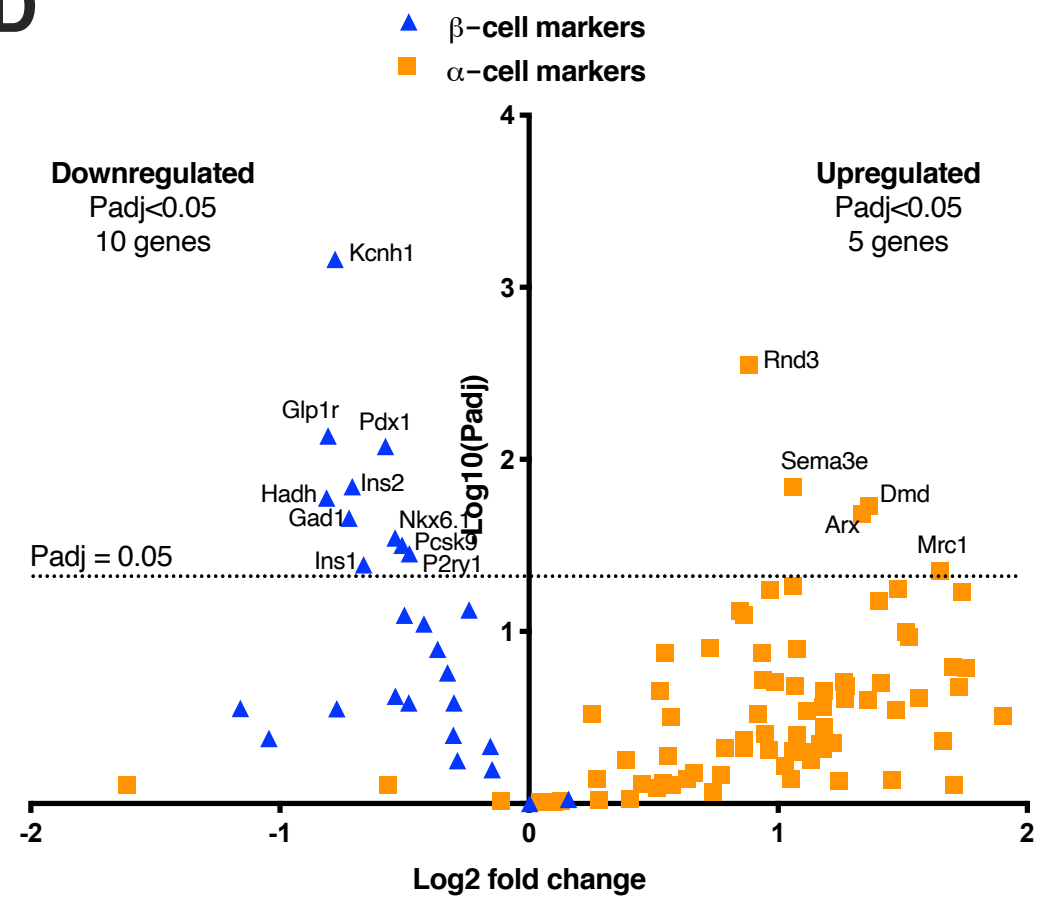

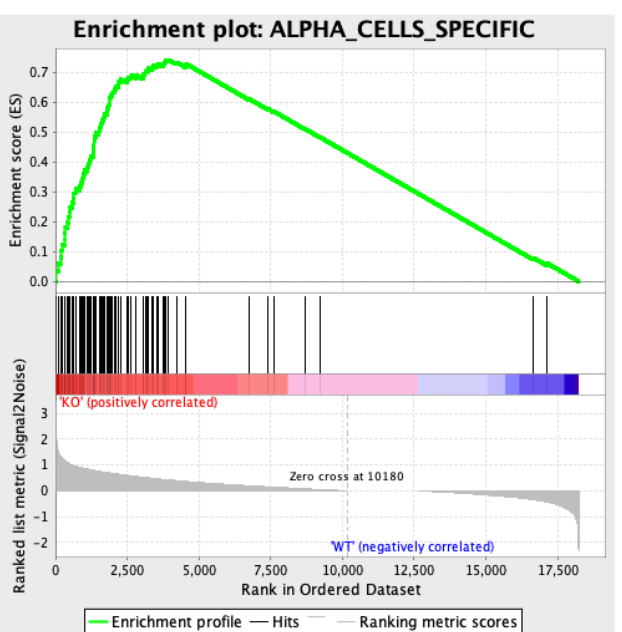

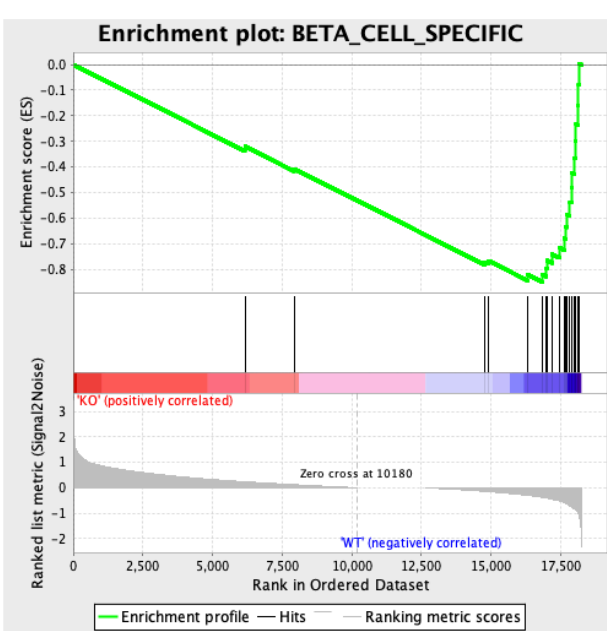


A

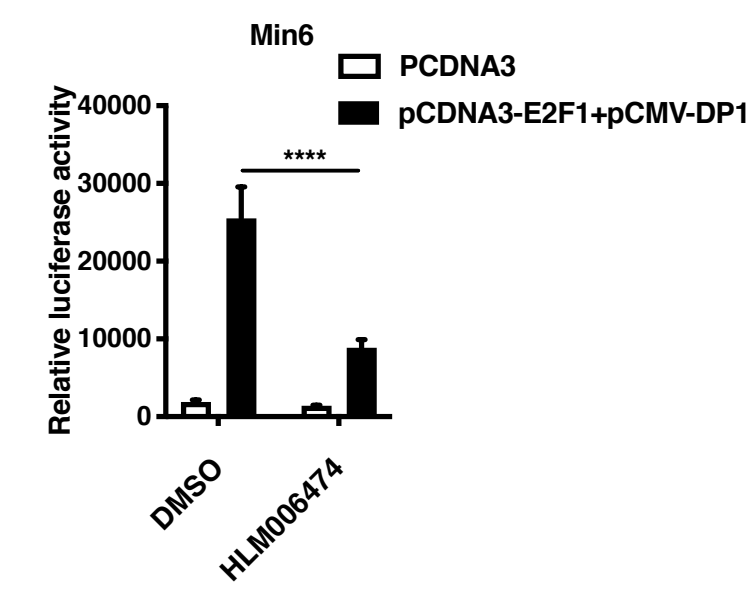

D

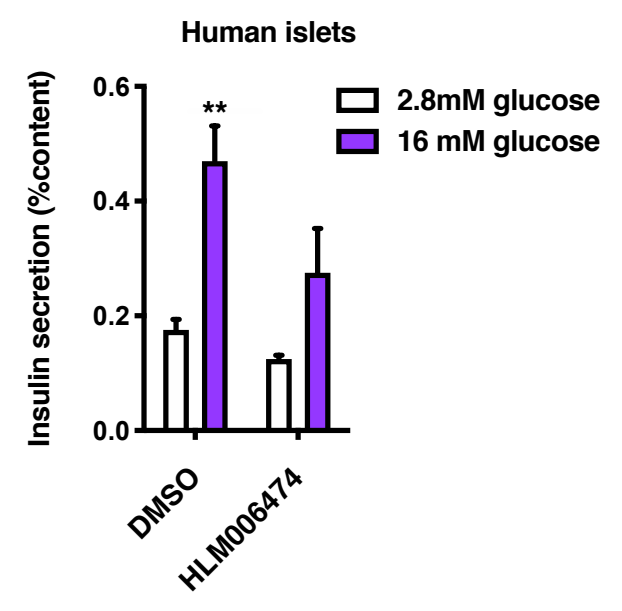

B

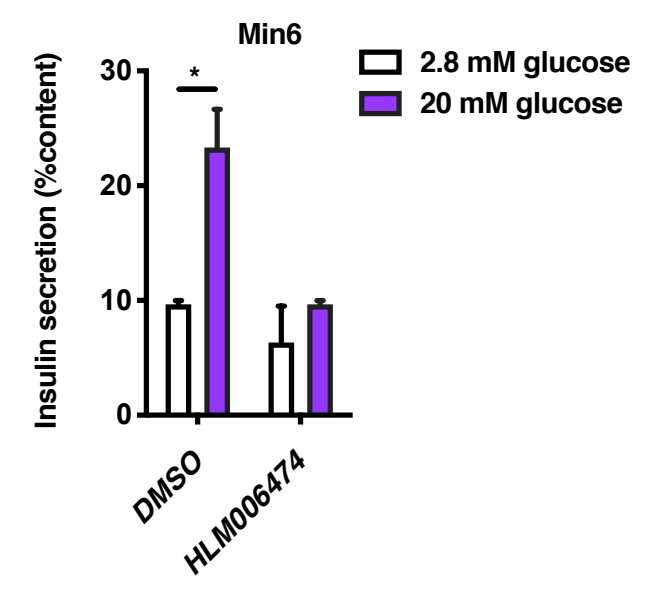

E

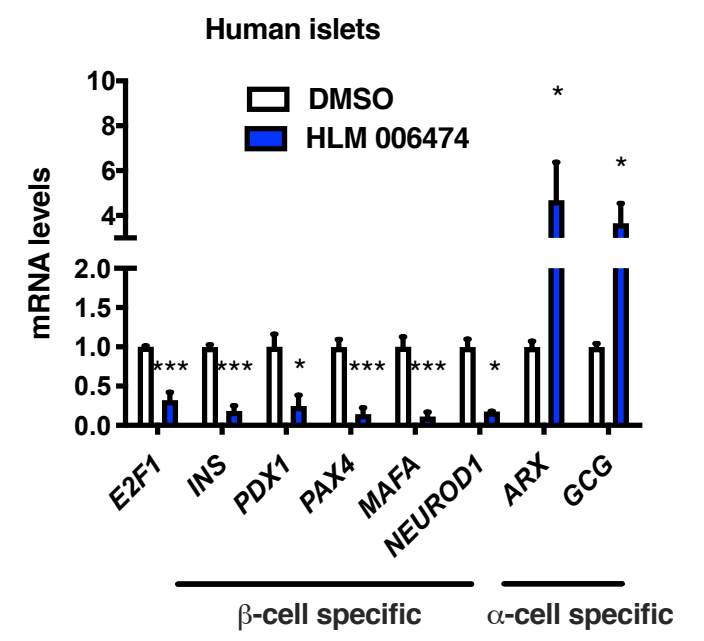

E
C

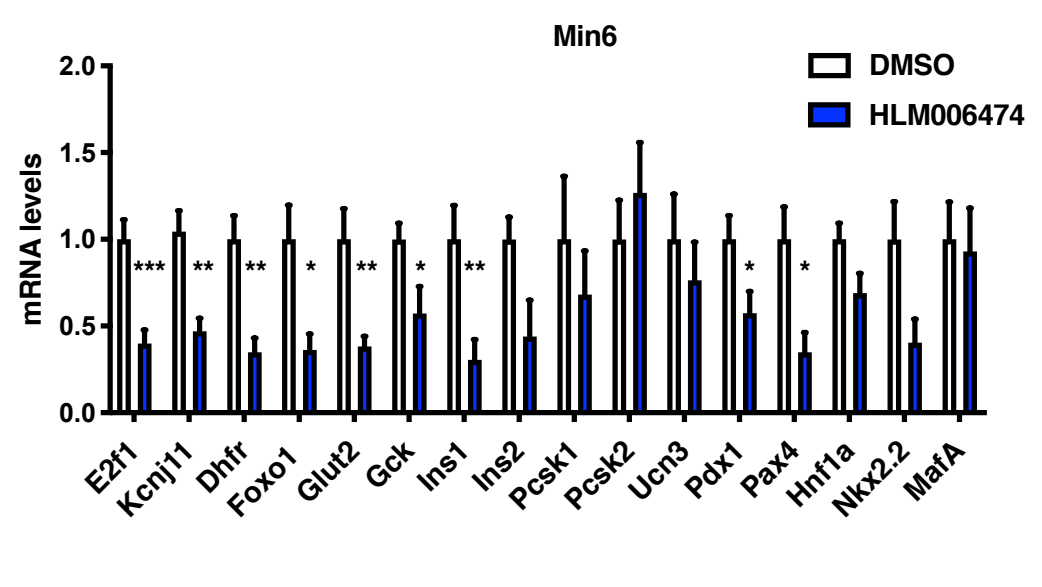


A

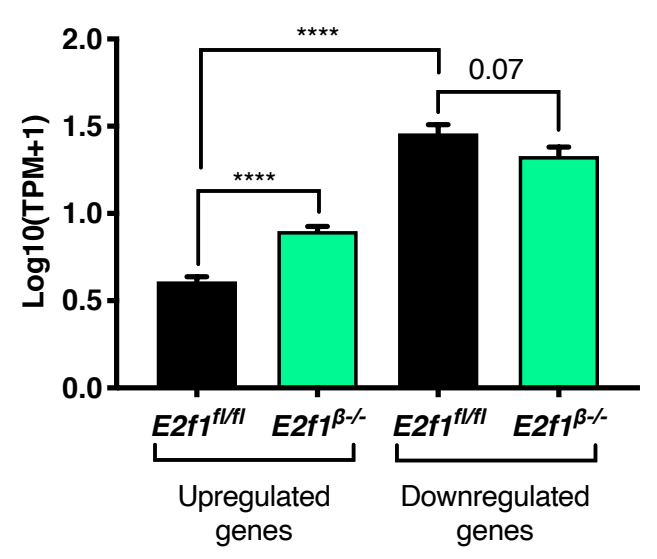

D
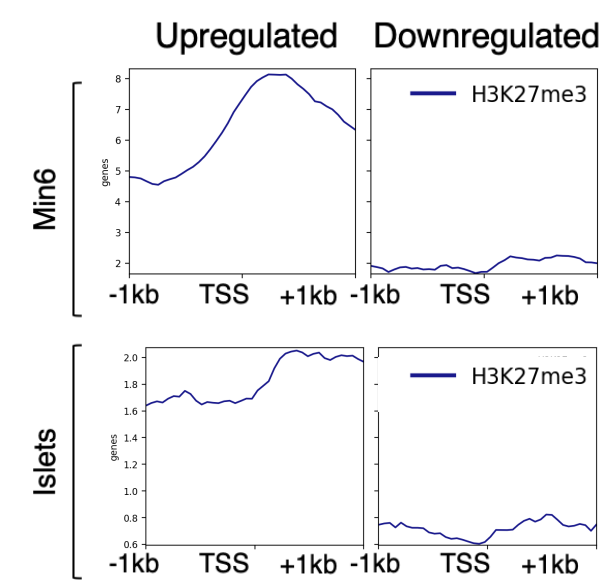

B

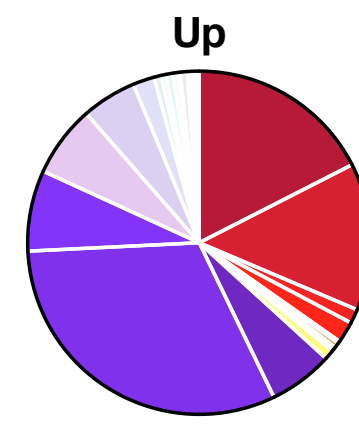

Down

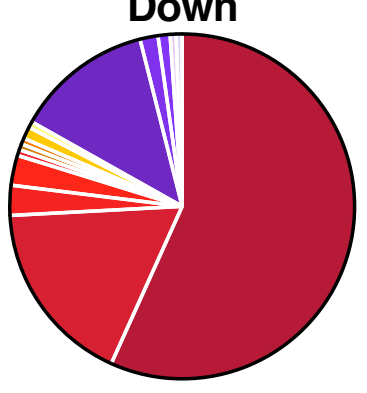

E

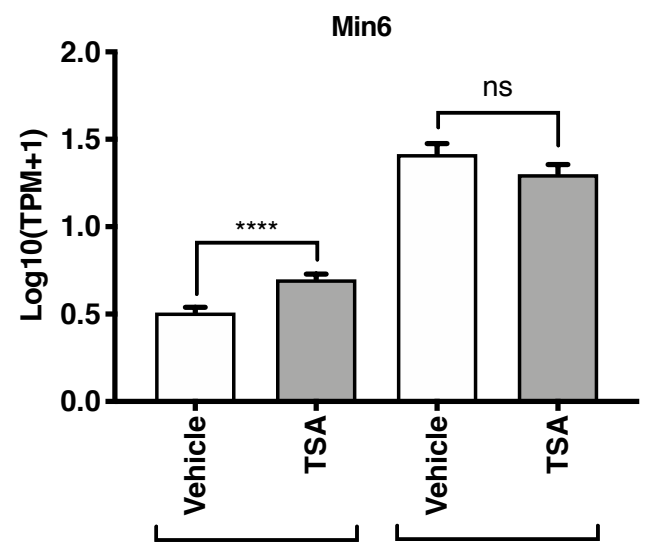

Upregulated genes Downregulated genes
C

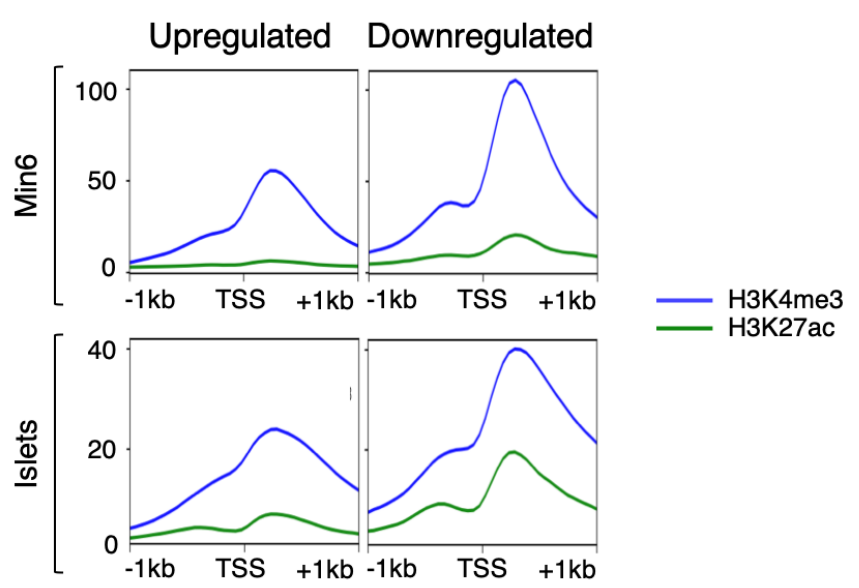

F

Min6

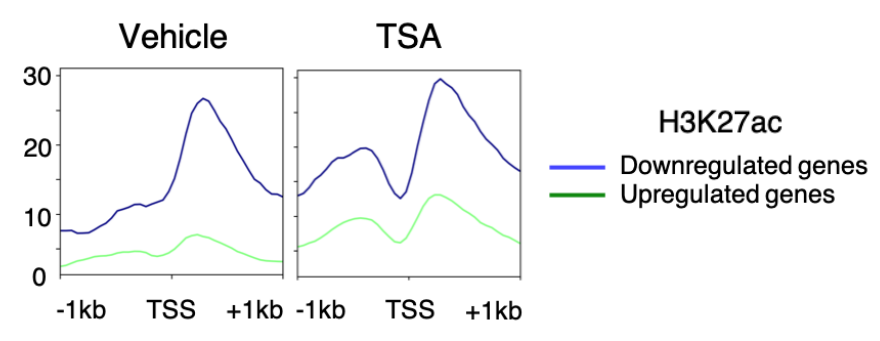

G

$\begin{array}{lll}\text { Vehicle Min6 } & \text { TSA }\end{array}$

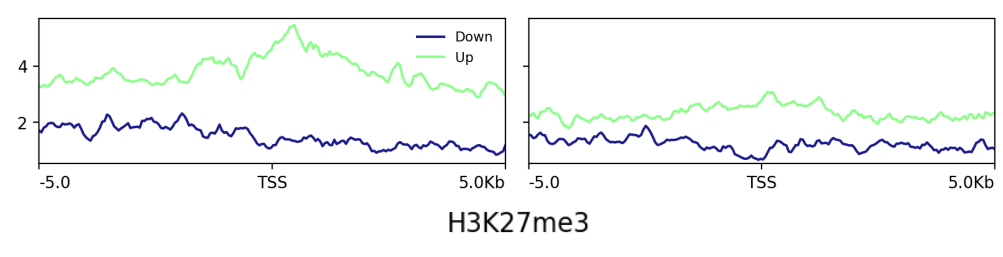

- Downregulated genes

Upregulated genes 


\begin{tabular}{|c|c|c|c|c|c|c|c|c|}
\hline \multicolumn{3}{|c|}{ If defficiency. } & & & & & & \\
\hline Upstream Regulator & Expr Log Ratio & Molecule Type & Predicted Activation Statf & Activation z-score & Flags & p-value of vertap & Target Molecules in Dataset & Mechanistic Network \\
\hline HNF1A & & transcription regulator & Innibited & $-4,293$ & bias & $8,22 E-10$ & ADH1C,ANPEP, CASC4,CCND1,COX6A2,ETV5,GLP1R,GNG12,INS, Ins1 1 & $43(9)$ \\
\hline XBP1 & & transcription regulator & Inhibited & $-2,417$ & bias & $9,70 \mathrm{E}-10$ & 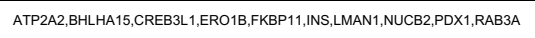 & $16(2)$ \\
\hline 2-oxoadipic acid & & chemical-endogenous mammalian & Inhibited & $-2,236$ & bias & $4,65 E-09$ & GLP1R,INS,Ins 1,PCLO,SYTL4 & \\
\hline 2-aminoadipic acid & & chemical- endogenous mammalian & Inhibited & $-2,236$ & bias & $4,65 E-09$ & GLP1R,INS,Ins1,PCLO,SYTL4 & \\
\hline DHTKD1 & & enzyme & & & & $1,68 E-08$ & GLP1R,INS,Ins1,PCLO,SYTL4 & \\
\hline tunicamycin & & hemical- endogenous non-mammalia & & $-1,737$ & bias & $1,96 \mathrm{E}-08$ & ATP2A2,CCND1,ERO1B,FKBP11,INS,LLMAN1,MANF,MLXIPL,PROM1,SDF2L1 & $19(4)$ \\
\hline $\mathrm{PDX} 1$ & $-0,576$ & transcription regulator & Innibited & $-2,530$ & bias & $6,53 E-08$ & ATP2A2, CCND1,ERO1B,FFAR1, GLP1R,,GNG 12,1NS, Ins 1,NKX6-1,PDX1 & \\
\hline NEUROG3 & & transcription regulator & Inhibited & $-2,613$ & bias & 3.05E-06 & DLL4,INS,Ins1,NKX6-1,NTRK2,PDX1,SYP & $32(10)$ \\
\hline NKX2-2 & & transcription regulator & & & & $4,36 E-06$ & INS, nns $1, N K X 6-1, \mathrm{PDX} 1$ & 7(4) \\
\hline dithiothreitol & & chemical reagent & & $-1,131$ & bias & 4,56E-06 & ATP2A2,CCND1,ERO1B,INS,LMAN1 & $8(2)$ \\
\hline FFAR3 & & G-protein coupled receptor & Activated & 2,630 & 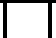 & $5,56 E-06$ & ANPEP,CA10,ns1, KCNH1,NECAB2,PCSKG,PITPNM3 & \\
\hline ERCC1 & & enzyme & & & & $1,12 E-05$ & GHR,Ins1,PRLR & \\
\hline PAX6 & & transcription regulator & & $-1,790$ & & $1,15 E-05$ & CCND1,FFAR1, GLP1R,INS, Ins 1,NECAB2,NNKX6-1,PDX1,TCEALG,WLS & $13(5)$ \\
\hline ATFG & & transcription regulator & & & 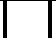 & 1,41E-05 & ATP2A2,INS,LMAN1,MANF,NUCB2,PDX 1 & \\
\hline NEUROD1 & & transcription regulator & Innibitied & $-2,187$ & bias & $1,73 E-05$ & INS, Ins1,NKX6-1,NTRK2,SYP & \\
\hline INSM1 & & transcription regulator & & & & $1,94 \mathrm{E}-05$ & INS,NKX6-1,PDX1 & \\
\hline ERN1 & & kinase & Innibitied & $-2,135$ & bias & $2,21 E-05$ & ATP2A2,ERO18,FKBP11,MLXXPL,SDF2L1,SPCS2,SRPRA,WFS1 & $17(3)$ \\
\hline GLIS3 & $-0,401$ & transcription regulator & & & & $2,49 E-05$ & INS, Ins $1, N \times x \in-1, P D \times 1$ & $30(7)$ \\
\hline IRS2 & & enzyme & & $-0,223$ & & $2,51 E-05$ & ACLY,CCND1,INS,Ins1,PDX1 & $18(4)$ \\
\hline sst & & other & & 0,651 & & 3,16E-05 & CCND1,GHR,INS,Ins1,PRLR & 19(4) \\
\hline D-glucose & & chemical-endogenous mammalian & & $-1,474$ & bias & $3,67 \mathrm{E}-05$ & ACLY,ADARB1,ATP2A2, CCND1, COXGA2,DLL4,ERO1B,FFAR1,GALR1,INS & $33(11)$ \\
\hline СD38 & & enzyme & Innibited & $-2,804$ & bias & $6,30 \mathrm{E}-05$ & CHST12,Ins1,MANF,NDUFV3,PKIB,PYCR1,SDF2L1,SPCS2 & \\
\hline PTPRF & & phosphatase & & & & $6,90 \mathrm{E}-05$ & CCND1,Ins1 & \\
\hline MAFA & & transcription regulator & & & & $8,92 E-05$ & INSSIns, PDX1 & \\
\hline PCSK2 & & peppidase & & & & $1,18 E-04$ & Ins1,NKX6-1,PDX1 & \\
\hline PRLR & $-0,983$ & transmembrane receptor & & $-0,781$ & bias & $1,23 E-04$ & GHR,IGFBP5,Ins1,VDR & \\
\hline THRA & & ligand-dependent nuclear receptor & & $-0,536$ & & $1,27 E-04$ & ATP2A2,CCND1,FRZB,Ins1,PDX1,PROM1,RCAN2 & \\
\hline TO-901317 & & chemical reagent & & $-1,294$ & bias & $1,73 E-04$ & ACLY,ADH1C,CCND1,GHR,INS,MLXIPL,PCSKG,PDX1,SYP & $33(9)$ \\
\hline rosigilitazone & & chemical drug & Innibited & $-2,750$ & bias & 1,79E-04 & 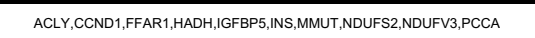 & 22 (4) \\
\hline MGATAA & & enzyme & & & 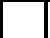 & $2,06 E-04$ & CCND1,ns1 & \\
\hline diethylstilibestrol & & chemical drug & & $-0,478$ & bias & $2,85 E-04$ & ADH1C,CCND1,CD200,DLG3,FRZB,MNS1,MuCA,NTRK2,PROM1,VDR & \\
\hline SPOP & & other & & & & $2,94 \mathrm{E}-04$ & INS:Ins, PDX1 & $12(2)$ \\
\hline АСACB & & enzyme & & & 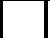 & $2,94 E-04$ & ACLY,Ins1,MLXIPL & $30(7)$ \\
\hline Ins1 & $-0,664$ & other & & $-1,041$ & bias & $3,07 \mathrm{E}-04$ & ADH1C,BPGM,CCND1,IGFBPP,INS,InS1,LGALL12,MLXXIPL,PRLR,SYP & $41(10)$ \\
\hline
\end{tabular}




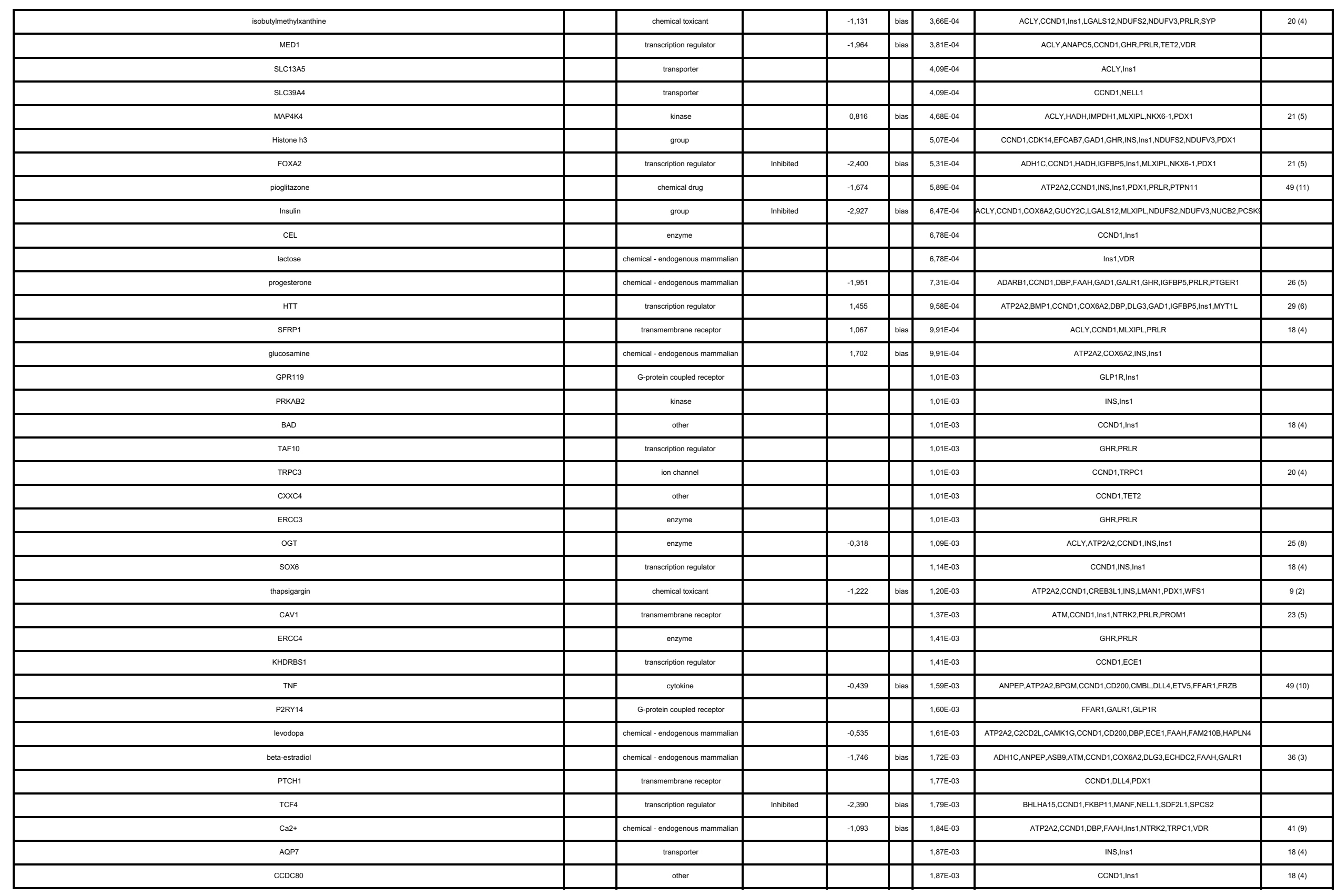




\begin{tabular}{|c|c|c|c|c|c|c|c|c|}
\hline SLC2A2 & & transporter & & & & 1,87E-03 & Inst,PDX1 & \\
\hline NFATC1 & & transcription regulator & Inhibited & $-2,213$ & bias & $1,87 \mathrm{E}-03$ & CCND1,INS,Ins1,PDX1,PRLR & $24(3)$ \\
\hline NRAA1 & & ligand-dependent tuclear receptor & & 1,673 & bias & 1,93E-03 & ACLY,BPGM,CCND1,NSS,Ins1,NDUFV3,NUCB2,PDX1 & $33(7)$ \\
\hline Gск & & kinase & & & & 1,95E-03 & ACLY,HADH,Ins 1 & \\
\hline sumo1 & & enzyme & & & & 1,95E-03 & ATP2A2,CCND1,PDX1 & $30(6)$ \\
\hline TCF3 & & transcription regulator & Inhibited & $-2,736$ & bias & $2,09 E-03$ & BHLHA15,FKBP11,1NS,Ins1,MANF,NELL1,NTRK2,SDF2L1,SPCS2 & \\
\hline BSCL2 & & other & & & & $2,12 E-03$ & CCND1,IGFBP5,Ins1,LGALS12 & \\
\hline D103 & & enzyme & & & & $2,15 E-03$ & INS, Ins 1, PXX1 & \\
\hline GPBAR1 & & G-protetin coupled recepptor & & & & 2,39E-03 & CCND1,Ins1 & \\
\hline SHANK3 & & other & & & & $2,39 E-03$ & PCLO,SYP & \\
\hline EPAS1 & & transcription regulator & & & & $2,72 E-03$ & ACLY,CCND1,IGFBP5,MANF,MYOTA,P2RY1,PKIB & \\
\hline PHF21A & & other & & & & $2,976-03$ & RABBA,SYP & \\
\hline TAF5 & & transcription regulator & & & & $2,97 E-03$ & GHR,PRLR & \\
\hline LDB1 & & transcription regulator & & & & $2,976-03$ & FRZB,Ins1 & \\
\hline SETD7 & & enzyme & & & & $2,976-03$ & CCND1,ins1 & 12(2) \\
\hline RANBP9 & & other & & & & $2,97 \mathrm{E}-03$ & RABBA,SYP & \\
\hline RELN & & peptidase & & & & $2,97 \mathrm{E}-03$ & GAD1,NTRK2 & \\
\hline STK11 & & kinase & & $-0,820$ & & $3,00 E-03$ & CCND1,HIIRA,IGFBP5,NOD1,NPEPL1,PAPSS1,POLD2,PYCR1 & \\
\hline Ртн & & other & & $-1,116$ & bias & 3,15E-03 & ATP2A2,CCND1,GNG 12,IGFBP5,PPP1R1A,VDR & $35(7)$ \\
\hline Growth hormone & & group & Inhibited & $-2,404$ & bias & $3,22 E-03$ & CCND1,GHR,HADH,IGFBP5,Ins1,PRLR,RAB3A & $37(6)$ \\
\hline NTRK2 & $-0,677$ & kinase & & & . & $3,57 \mathrm{E}-03$ & CCND1,GAD1,RAB3A,SYP & \\
\hline PTTG1 & & transcription regulator & & 1,067 & bias & $3,57 \mathrm{E}-03$ & ATM,CCND1, Ins 1, TKK1 & \\
\hline TRH & & other & & & & $3,58 \mathrm{E}-03$ & CDK14,Ins1,PRLR & \\
\hline cIC & & transcription regulator & & & & $3,611-03$ & CCND1,ETV5 & \\
\hline VDR & $-0,679$ & transcription regulator & & $-1,698$ & & $3,89 \mathrm{E}-03$ & ATM,CCND1,HIRA,IGFBP5, InS1,TLK1,VDR & $34(6)$ \\
\hline CYP19A1 & & enzyme & & $-1,624$ & & $3,922-03$ & CCND 1,lns1,PRLR,SYP & \\
\hline metribolone & & chemical reagent & & $-1,746$ & bias & $4,06 \mathrm{E}-03$ & 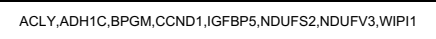 & \\
\hline cyclopamine & & chemical reagent & & & & $4,17 E-03$ & CCND1,NKX6-1,PDX1 & \\
\hline PTPN11 & $-0,341$ & phosphatase & & $-1,154$ & & $4,30 \mathrm{E}-03$ & ATP2A2,CCND1,ns1,PTPN11 & $31(4)$ \\
\hline SREBF2 & & transcripition regulator & & $-0,192$ & bias & 4,30E-03 & ACLY,Ins,PCSK, PDX1 & $18(4)$ \\
\hline LAMTOR5 & & other & & & & $4,31 E-03$ & INS, Ins1 & \\
\hline NUMBL & & other & & & & $4,31 E-03$ & DLL4,VDR & \\
\hline CCNT1 & & transcription regulator & & & & $4,31 E-03$ & CCND1,INS & \\
\hline РІКзСВ & & kinase & & & & $4,31 E-03$ & DLL4,1ns1 & \\
\hline myristoylated protein kinase $A$ inhibitor $14-22$ amide & & chemical- kinase inhibitor & & & & $4,31 E-03$ & CCND1,PDX1 & $12(2)$ \\
\hline indomethacin & & chemical drug & & 0,168 & bias & 4,45E-03 & ABCD3,,CCND1,MANF,PRLR,PROM1,TNFRSF21,VDR & \\
\hline tacrolimus & & chemical drug & Activated & 2,425 & bias & $4,86 E-03$ & ABCD3,CCND1,CD200,INS,Ins1,PROM1,WLS & $42(6)$ \\
\hline
\end{tabular}




\begin{tabular}{|c|c|c|c|c|c|c|c|c|}
\hline GHR & $-0,488$ & transmembrane receptor & & & & $5,14 E-03$ & GHR,Ins1,PRLR & \\
\hline SREBF1 & & transcription regulator & & $-1,354$ & bias & $5,41 E-03$ & ACLY,ADH1C,INS,Ins1,NKX6-1,PCSK9,PDX1 & 31 (9) \\
\hline FGF19 & & growth factor & & & & 5,58E-03 & ACLY,CCND1,PROM1,SLC2A5 & \\
\hline MAFB & & transcription regulator & & & & $5,81 E-03$ & INS Ins 1, NKX6-1,PDX1 & \\
\hline moxonidine & & chemical drug & & & & $5,88 E-03$ & ANPEP,P2RY1 & \\
\hline NAE 1 & & enzyme & & & & $5,88 E-03$ & CCND1,CDK5RAP1 1 & \\
\hline NEUROG2 & & transcription regulator & & & & $5,88 E-03$ & INS,NKX6-1 & \\
\hline PER2 & & transcription regulator & & & & $5,88 E-03$ & CCND1,DBP & \\
\hline cyproterone acetate & & chemical drug & & & & $5,88 E-03$ & CCND1,PRLR & $7(2)$ \\
\hline A2M & & transporter & & & & $6,24 E-03$ & ACLY,CCND1,PRLR & \\
\hline hydrocortisone & & chemical- endogenous mammalian & & $-1,503$ & & 6,59E-03 & CCND 1,GGBPF, INS,NTRK2,PRLR & $32(4)$ \\
\hline roscovitine & & chemical drug & & & & 6,63E-03 & CCND1, nns1,PDX1 & $21(5)$ \\
\hline ADAM10 & & peptidase & & & & $6,82 E-03$ & ATP2A2,CCND1,SYP,TRIM9 & \\
\hline IFNG & & cytokine & & 1,144 & bias & $6,95 E-03$ & ABCD3,ACLY,AQP11,ATP2A2, СCND1,CD200,DBP, „ECE1,FAAH,FFAR1 & $61(12)$ \\
\hline FGF21 & & growth factor & & & & 7,04E-03 & ACLY,GHR,Ins 1 & \\
\hline phosphate & & chemical-endogenous mammalian & Activated & 2,000 & bias & $7,09 E-03$ & ATM,CHST12,PCCA,SDF2L1 & \\
\hline FGF8 & & growhth factor & & $-0,468$ & bias & 7,36E-03 & CCND1,DUSP23,ETV5,VDR & \\
\hline MED13 & & transcription regulator & & & . & $7,46 E-03$ & BPGM,NTRK2,PCSK9 & \\
\hline FMR1 & & translation regulator & & 1,340 & bias & $7,61 \mathrm{E}-03$ & DLG3,NPEPL1,PCLO,RABBA,SYP & \\
\hline KLF9 & & transcription regulator & & & & $7,67 \mathrm{E}-03$ & CCND1,GHR & \\
\hline$N K \times 6-1$ & $-0,538$ & transcription regulator & & & & $7,67 \mathrm{E}-03$ & $\operatorname{Ins} 1, \mathrm{PDX} 1$ & \\
\hline NDN & & transcription regulator & & & & $7,67 \mathrm{E}-03$ & NUCB2,SYP & \\
\hline nilotinib & & chemical drug & & & & $7,90 \mathrm{E}-03$ & АВСD3,АTM,PTPN11 & \\
\hline Histone h4 & & group & & & & 8,05E-03 & CCND1,NS, NTRK2,POLD2,PRLR & \\
\hline NCOA1 & & transcription regulator & & 0,762 & bias & $8,05 E-03$ & BPGM,CCND1,DBP,HIRA,PRLR & \\
\hline PPARG & & ligand-dependent nuclear receptor & Inhibited & $-2,850$ & bias & $8,18 \mathrm{E}-03$ & ACLY,ATP2A2, CCND1,FFAR1,IGFBP5, INS ,Ins1,PCLO,PDX1,VDR & $47(9)$ \\
\hline HMGA1 & & transcription regulator & & $-1,452$ & bias & $8,27 E-03$ & ATM,CCND1,COX6A2,GHR,/ns1 & \\
\hline ALG10 & & enzyme & & & & $8,33 E-03$ & PROM1 & \\
\hline DRAXIN & & other & & & & $8,33 E-03$ & Ins1 & \\
\hline dichlobeniil & & chemical toxicant & & & & $8,33 E-03$ & GAD1 & \\
\hline Ces1b/Ces1c & & enzyme & & & & $8,33 E-03$ & GHR & \\
\hline NCK & & group & & & & $8,33 E-03$ & CCND1 & \\
\hline CYP2R1 & & enzyme & & & & $8,33 E-03$ & VDR & \\
\hline моGs & & enzyme & & & & $8,33 E-03$ & PROM1 & \\
\hline Pritd & & cytokine & & & & $8,33 E-03$ & PRLR & \\
\hline ENHO & $-0,613$ & other & & & & $8,33 E-03$ & Ins1 & \\
\hline FBX031 & & enzyme & & & & $8,33 E-03$ & CCND1 & \\
\hline
\end{tabular}




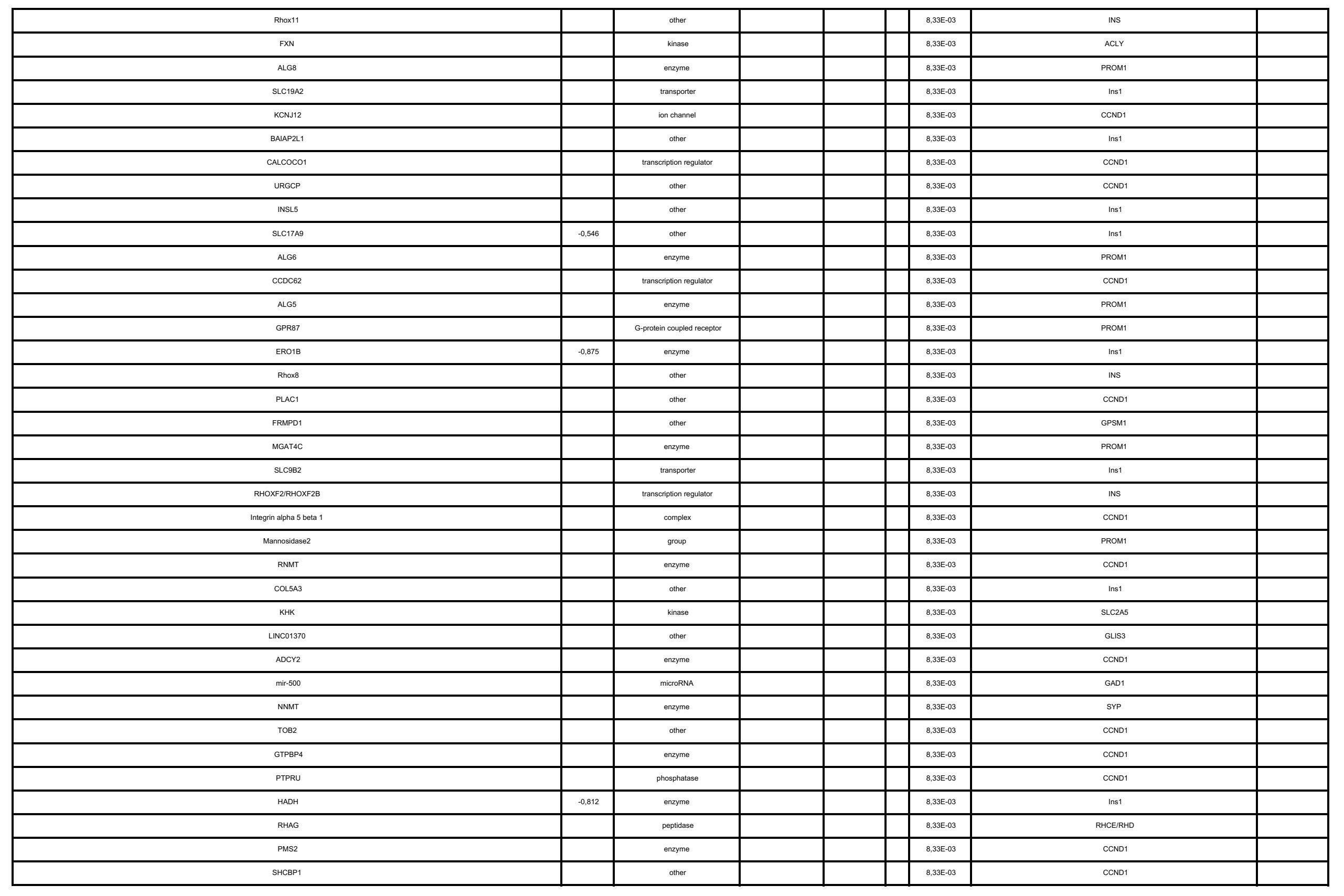




\begin{tabular}{|c|c|c|c|c|c|c|c|}
\hline ABCG5 & transporter & & & & $8,33 E-03$ & INS & \\
\hline INSRR & kinase & & & & $8,33 E-03$ & Ins1 & \\
\hline $1 \mathrm{TTH} 4$ & other & & & & $8,33 E-03$ & VDR & \\
\hline Сок20 & kinase & & & & $8,33 E-03$ & CCND1 & \\
\hline PTOV1 & other & & & & $8,33 E-03$ & CCND1 & \\
\hline PASK & kinase & & & & $8,33 E-03$ & $\mathrm{PDX} 1$ & \\
\hline ARFGEF3 & other & & & & $8,33 E-03$ & Ins1 & \\
\hline PNLIP & enzyme & & & & $8,33 E-03$ & Ins 1 & \\
\hline RPLPO & other & & & & $8,33 E-03$ & CCND1 & \\
\hline amlexanox & chemical drug & & & & $8,33 E-03$ & CCND1 & \\
\hline sulfaphenazole & chemical drug & & & & $8,33 E-03$ & CCND1 & \\
\hline Ro 20-1724 & chemical reagent & & & & $8,33 E-03$ & CCND1 & \\
\hline anti-miR-302d & chemical reagent & & & & $8,33 E-03$ & CCND1 & \\
\hline anti-miR-302c inhibitor & chemical reagent & & & & $8,33 E-03$ & CCND1 & \\
\hline beta-penta-O-galloy-glucose & hemical - endogenous non-mammaila & & & & $8,33 E-03$ & CCND1 & \\
\hline formycin B & chemical reagent & & & & $8,33 E-03$ & Ins1 & \\
\hline monomethyl succinate & chemical reagent & & & & $8,33 E-03$ & Ins1 & \\
\hline 10-hydroxystearic acid & hremical - endogenous non-mammalia & & & & $8,33 E-03$ & FFAR1 & \\
\hline NMN & chemical- endogenous mammalian & & & & $8,33 E-03$ & $\mathrm{PDX} 1$ & \\
\hline fish oils & chemical drug & & & & $8,36 E-03$ & ABCD3,MLXXPL,NUCB2 & \\
\hline Hedgenog & group & & & & $8,36 E-03$ & CCND1,NKX6-1,PDX1 & \\
\hline CTNNB1 & transcription regulator & Inhibited & $-2,183$ & \begin{tabular}{|l|l|} 
bias \\
\end{tabular} & $8,45 E-03$ & ACLY,ATM,BMP1,CASC4,CCND1,DLL4,GAD1,GHR,IFT57,IGFBP5 & $17(2)$ \\
\hline daidzein & chemical drug & & & & $8,53 E-03$ & CCND1,PKIB,PRLR,SYP & $16(3)$ \\
\hline calcitiol & chemical drug & & 1,329 & & $8,54 \mathrm{E}-03$ & ATM,CCND1,ETVS,GHR, IGFBP5, INS Ins1, KCNH1,PYCR1, TLK1 & $44(7)$ \\
\hline MAZ & transcription regulator & & & & $8,64 \mathrm{E}-03$ & INS, Ins1 & \\
\hline pyruvaldehyde & chemical-endogenous mammalian & & & & $8,64 \mathrm{E}-03$ & Ins1,PDX1 & \\
\hline ARNTL & transcription regulator & & & & $8,83 E-03$ & DBP,Ins1,MLXIPL & \\
\hline PGR & ligand-dependent nuclear receptor & & $-1,791$ & bias & $9,04 E-03$ & ADARB1, CCND 1, IGFBP5, Ins1,PRLR, TNFRSF21,VDR & $20(4)$ \\
\hline torin1 & chemical reagent & & 1,982 & bias & $9,16 \mathrm{E}-03$ & ADH1C,BPGM,CCND1,NDUFS2 & \\
\hline CREM & transcription regulator & & 0,391 & & $9,46 \mathrm{E}-03$ & ATP2A2,CCND1,1NS,Ins1,MNS1 & $19(4)$ \\
\hline clofibrate & chemical drug & & $-0,152$ & bias & $9,48 E-03$ & ABCD3,CCND1, РCSK9,SLCO281 & \\
\hline HIF $1 \mathrm{~A}$ & transcription regulator & & $-1,950$ & bias & $9,62 E-03$ & ATP2A2,CCND1, DLL4,GHR,IGFBP5,MANF,NTRK2,PROM1,TRIM66 & $22(3)$ \\
\hline NMDA Receptor & complex & & & & $9,67 \mathrm{E}-03$ & NTRK2,SYP & \\
\hline GLIS2 & transcription regulator & & & & $9,67 \mathrm{E}-03$ & CCND1,INS & \\
\hline TAF1 & transcription regulator & & & & $9,67 \mathrm{E}-03$ & GHR,PRLR & \\
\hline ATR & kinase & & & & $9,67 \mathrm{E}-03$ & АTM,CDK14 & \\
\hline RPS6KA3 & kinase & & & & $9,67 \mathrm{E}-03$ & INS, Ins1 & $8(2)$ \\
\hline
\end{tabular}




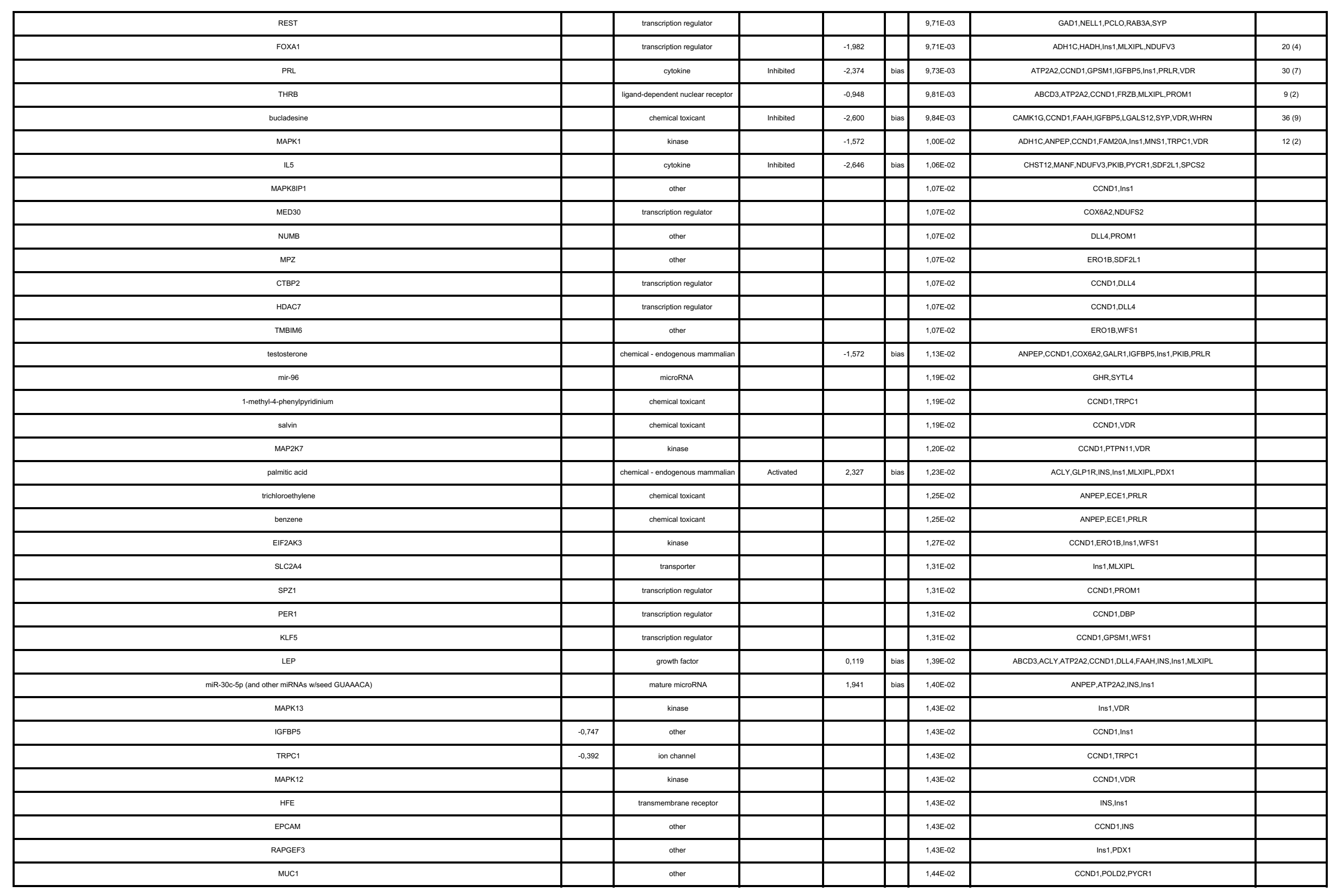




\begin{tabular}{|c|c|c|c|c|c|c|}
\hline troglitazone & chemical drug & $-0,006$ & & 1,44E-02 & BMP1,CCND 1,INS,Ins1,MLXIPL,NKXX-1,PDX1,PTPN11 & \\
\hline IL6ST & transmembrane receptor & & & $1,50 \mathrm{E}-02$ & ATP2A2, CCND1,MUC4 & \\
\hline ERBB3 & kinase & 0,152 & & $1,53 \mathrm{E}-02$ & CCND1,ETV5,GHR,IGFBP5 & \\
\hline miR-204-5p (and other miRNAs w/seed UCCCUUU) & mature microRNA & & & $1,56 \mathrm{E}-02$ & BMP1,קTPN11 & \\
\hline MBD1 & transcripition regulator & & & $1,56 \mathrm{E}-02$ & PROM1,PTPRM & \\
\hline DNMT3B & enzyme & 0,447 & bias & $1,57 \mathrm{E}-02$ & ATM,ATP2A2,MID1,PPP1R1A,RCAN2 & \\
\hline ethanol & chemical - endogenous mammalian & $-1,982$ & bias & $1,61 \mathrm{E}-02$ & ACLY,ADHIC,ANPEP,CCND1,FAAH,MLXXPL,NTRK2,RABBD & \\
\hline gefftinib & chemical drug & & & 1,61E-02 & CCND1,ETV5,GHR,IGFBP5,PTGER1 & \\
\hline guanosine & chemical - endogenous mammalian & & & $1,66 \mathrm{E}-02$ & Ins1 & \\
\hline isopropy thiogalactoside & chemical reagent & & & $1,66 \mathrm{E}-02$ & CCND1 & \\
\hline COLAA3BP & kinase & & & $1,66 \mathrm{E}-02$ & CCND1 & \\
\hline Ionaprisan & chemical drug & & & $1,66 \mathrm{E}-02$ & CCND1 & \\
\hline nitrosomethylurethane & chemical reagent & & & $1,66 \mathrm{E}-02$ & CCND1 & \\
\hline propionate derivative & chemical - other & & & $1,66 \mathrm{E}-02$ & MMUT & \\
\hline SLC5A1 & transporter & & & $1,66 \mathrm{E}-02$ & Ins1 & \\
\hline CACNA1E & ion channel & & & $1,66 E-02$ & Ins1 & \\
\hline IPGK1 & kinase & & & $1,66 \mathrm{E}-02$ & Ins1 & \\
\hline LCLAT1 & enzyme & & & $1,66 \mathrm{E}-02$ & Ins1 & \\
\hline SLC9A6 & transporter & & & $1,66 \mathrm{E}-02$ & NTRK2 & \\
\hline REM2 & enzyme & & & $1,66 \mathrm{E}-02$ & CCND1 & \\
\hline RPRD1B & other & & & $1,66 \mathrm{E}-02$ & CCND1 & \\
\hline $\operatorname{ccDC8BC}$ & other & & & $1,66 E-02$ & CCND1 & \\
\hline OS1-027 & chemical drug & & & $1,66 \mathrm{E}-02$ & CCND1 & \\
\hline SLC30AB & transporter & & & $1,66 \mathrm{E}-02$ & INS & \\
\hline VENTX & transcription regulator & & & $1,66 \mathrm{E}-02$ & CCND1 & \\
\hline GATM & enzyme & & & $1,66 \mathrm{E}-02$ & Ins1 & \\
\hline WDR13 & other & & & $1,66 \mathrm{E}-02$ & Ins1 & \\
\hline PARL & peptidase & & & $1,66 \mathrm{E}-02$ & тTC19 & \\
\hline EGFLG & other & & & $1,66 \mathrm{E}-02$ & PTPN11 & \\
\hline ETS-ELK1 & complex & & & $1,66 \mathrm{E}-02$ & CCND1 & \\
\hline CTNNB-LEF1 & complex & & & $1,66 E-02$ & CCND1 & \\
\hline SLURP1 & cytokine & & & $1,66 \mathrm{E}-02$ & Ins1 & \\
\hline UCN3 & other & & & $1,66 \mathrm{E}-02$ & Ins1 & \\
\hline EFFH & translation regulator & & & $1,66 \mathrm{E}-02$ & CCND1 & \\
\hline MCF2L & other & & & 1,66E-02 & CCND1 & \\
\hline SRI & transporter & & & $1,66 \mathrm{E}-02$ & Ins1 & \\
\hline RRAD & enzyme & & & $1,66 \mathrm{E}-02$ & CCND1 & \\
\hline
\end{tabular}




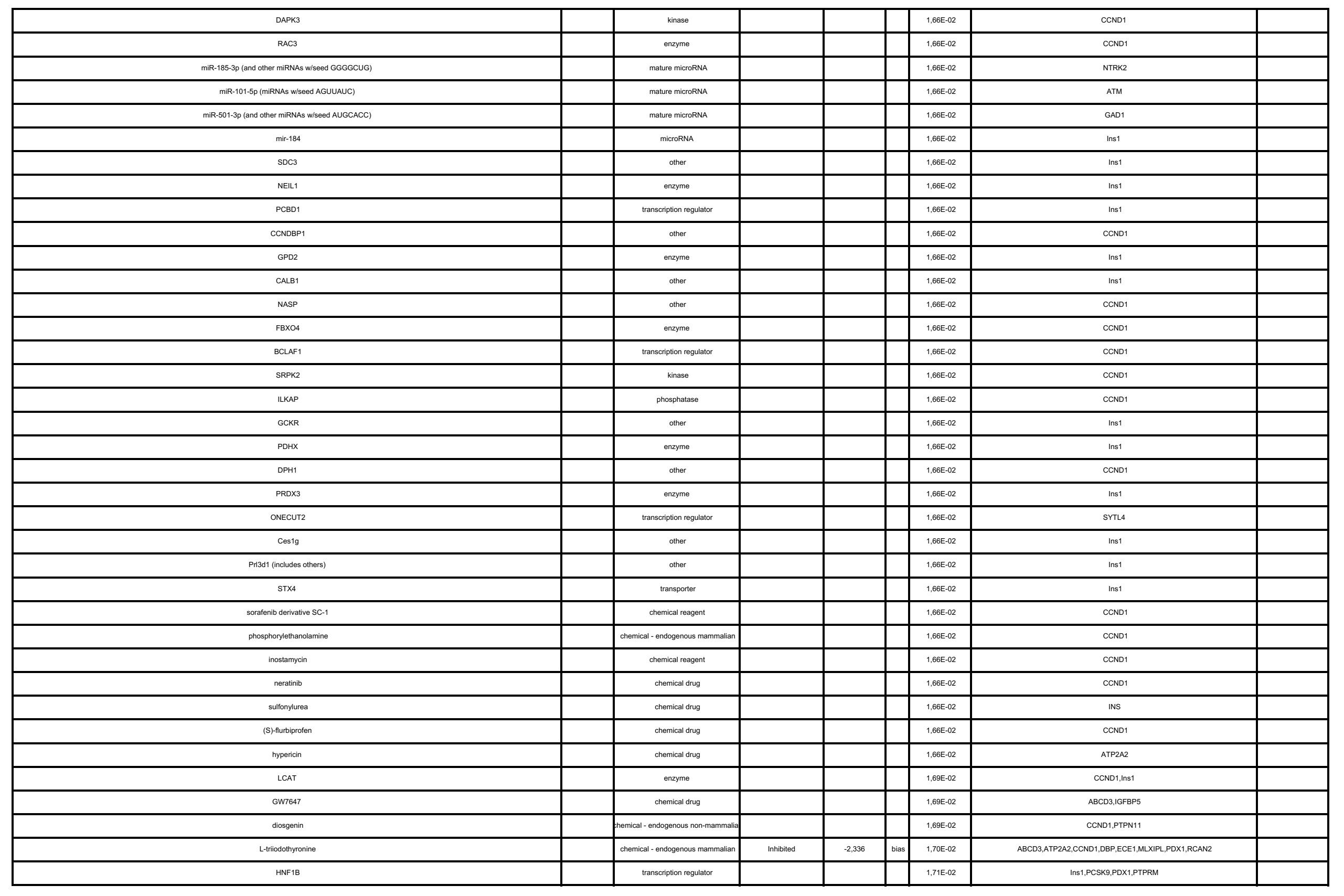




\begin{tabular}{|c|c|c|c|c|c|c|c|}
\hline TGFB1 & growth factor & & 1,327 & bias & $1,72 E-02$ & ACLY,ANPEP,AQP $11, \mathrm{ATM}, \mathrm{BMP} 1, \mathrm{CCND} 1, \mathrm{DBP}, \mathrm{ECE} 1, \mathrm{GAD} 1, \mathrm{GUCY} 2 \mathrm{C}$ & \\
\hline methylmercury & chemical toxicant & & & & 1,77E-02 & ANPEP,ECE1,PRLR & \\
\hline calpain & complex & & & & $1,82 E-02$ & CCND1,NTRK2 & \\
\hline BCL2L11 & other & & & & $1,82 E-02$ & CCND1,NOD1 & \\
\hline BTG2 & transcription regulator & & & & $1,82 E-02$ & CCND1,PDX1 & \\
\hline $\mathrm{NR2C2}$ & ligand-dependent nuclear receptor & & & & 1,82E-02 & CCND1, Ins 1 & \\
\hline SMARCA2 & transcription regulator & & & & $1,84 \mathrm{E}-02$ & IGFBP5,MYTIL,VDR & \\
\hline levothyroxine & chemical - endogenous mammalian & & & & $1,84 E-02$ & ATP2A2, CDK 14, MLXIPL & \\
\hline GکКзв & kinase & & 1,694 & & $1,86 E-02$ & ATP2A2,CCND1, Ins 1, PDX1 & \\
\hline staurosporine & chemical - kinase inhibitor & & 0,000 & & $1,86 E-02$ & CCND1,IGFBP5,Ins1,VDR & \\
\hline lithium chloride & chemical drug & & $-1,109$ & bias & $1,91 \mathrm{E}-02$ & ACLY,CCND1,DLL4,FAM135B & \\
\hline МАР2К2 & kinase & & & & 1,97E-02 & ETVS,INS & \\
\hline linolenic acid & chemical - endogenous mammalian & & & & $1,97 \mathrm{E}-02$ & $A B C D 3$, Ins1 & \\
\hline Raf & group & & & & 1,99E-02 & ANPER,CCND1,ETV5 & \\
\hline PITX2 & transcription regulator & & $-1,929$ & bias & $2,02 E-02$ & ADH1C,CCND1,GAD1,NDUFS2 & \\
\hline tetrodotoxin & chemical drug & & & & $2,11 \mathrm{E}-02$ & GAD1,NTRK 2 & \\
\hline PRKDC & kinase & & & & $2,11 \mathrm{E}-02$ & ATM,CCND1 & \\
\hline TCF7L2 & transcription regulator & Inhibited & $-2,580$ & bias & $2,15 E-02$ & CCND1,GLP1R,INS,Ins 1,PAPSS1,PDX1,PTPN11 & \\
\hline KLF3 & transcription regulator & & $-0,378$ & bias & $2,18 E-02$ & GAD1,GPSM1,HIST1142B, NDOUFY3,NOD1,TNFRSF21,UFSP2 & \\
\hline EGFR & kinase & & $-1,572$ & bias & $2,22 E-02$ & CCND1,FKBP11,GHR,HIRA,IGFBP5,PROM1,SDF2L1,VDR & \\
\hline FGF7 & growth factor & & & & $2,23 E-02$ & CCND1,ETVS,INS & \\
\hline sterol & chemical-endogenous mammalian & & & & $2,26 \mathrm{E}-02$ & ACLY,PCSK9 & \\
\hline bexarotene & chemical drug & & $-0,314$ & bias & $2,29 E-02$ & CCND 1, Ins1, NTRK2,PCSK9,SYP & \\
\hline E2F2 & transcription regulator & & & & $2,31 E-02$ & CCND1,ECE1, ns1 & \\
\hline POUSF1 & transcription regulator & & $-0,933$ & bias & $2,35 E-02$ & CCND1,FRZB,PDX1,PROM1,TET2,TNFRSF21,WLS & \\
\hline CRTC2 & other & & & & $2,42 E-02$ & CCND1, Ins 1 & \\
\hline CFLAR & other & & & & $2,42 E-02$ & Ins1,PDX1 & \\
\hline letrozole & chemical drug & & & & $2,42 E-02$ & CCND1,SYP & \\
\hline UM101 & chemical-kinase innibitior & & & & $2,42 E-02$ & CD200,VDR & \\
\hline 1-palmityyl-2-arachididnoyl-phosphatidyycholine & chemical-endogenous mammalian & & & & $2,48 E-02$ & CCND1 & \\
\hline myosin-light-chain kinase & group & & & & $2,48 E-02$ & CCND1 & \\
\hline FKHR & group & & & & $2,48 E-02$ & CCND1 & \\
\hline muraglitzzar & chemical drug & & & & $2,48 E-02$ & $\operatorname{lns} 1$ & \\
\hline NSD3 & enzyme & & & & $2,48 E-02$ & CCND1 & \\
\hline JUn-ATF2 & complex & & & & $2,48 E-02$ & CCND1 & \\
\hline FREM1 & other & & & & $2,48 E-02$ & FREM2 & \\
\hline FAM129B & transcription regulator & & & & 2,48E-02 & CCNO1 & \\
\hline
\end{tabular}




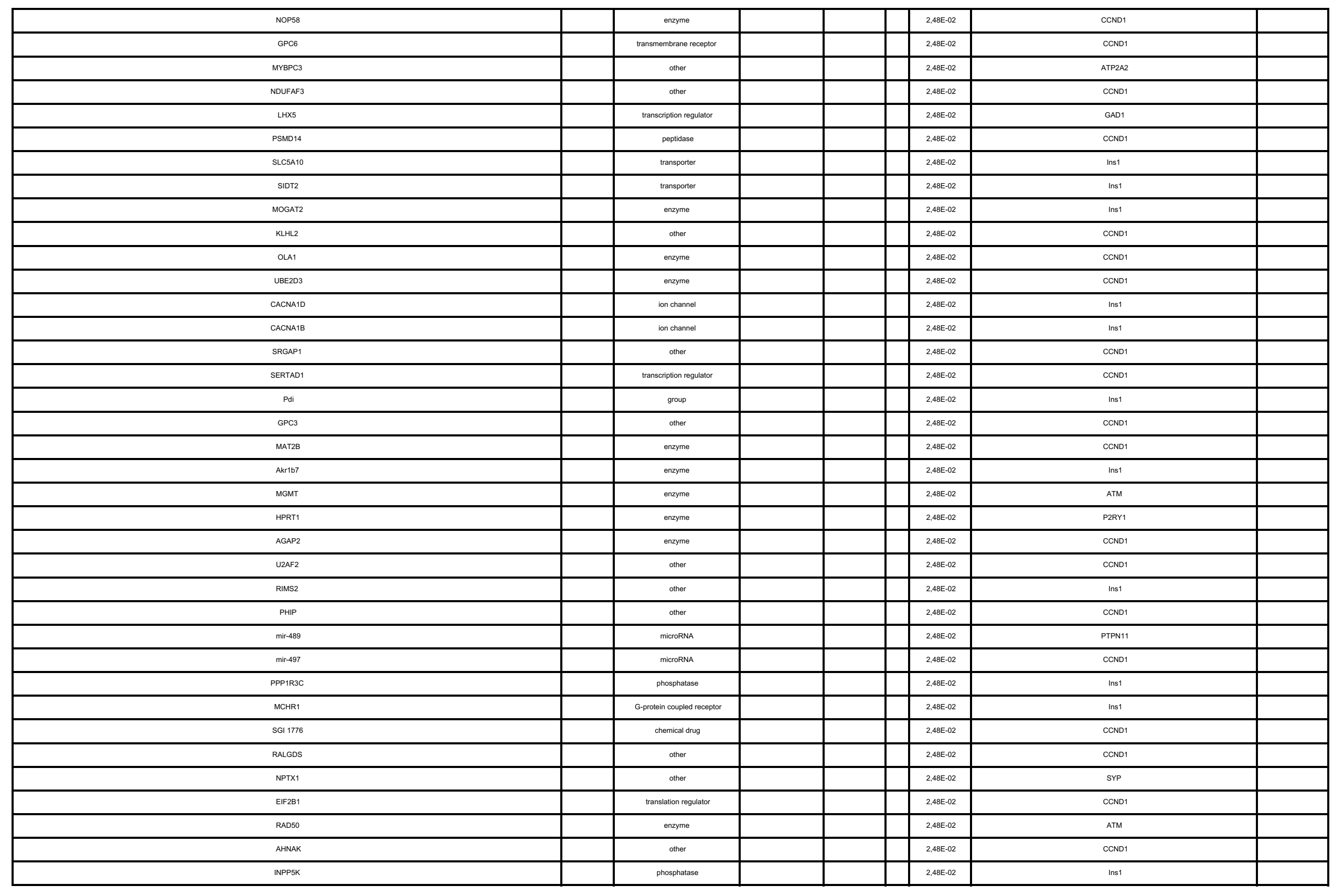




\begin{tabular}{|c|c|c|c|c|c|}
\hline BCAT2 & enzyme & & & $2,48 E-02$ & Ins1 \\
\hline KCNQ2 & ion channel & & & $2,48 E-02$ & SYP \\
\hline DDOST & enzyme & & & $2,48 E-02$ & PROM1 \\
\hline PTTGIP & other & & & $2,48 E-02$ & CCND1 \\
\hline MARK3 & kinase & & & $2,48 E-02$ & $\operatorname{lns} 1$ \\
\hline HELZ2 & transcription regulator & & & $2,48 E-02$ & Ins1 \\
\hline MBD5 & other & & & $2,48 E-02$ & Ins1 \\
\hline CTAGIACTAG1B & other & & & $2,48 E-02$ & CCND1 \\
\hline TBC1D4 & other & & & $2,48 E-02$ & Ins1 \\
\hline GOPC & transporter & & & $2,48 E-02$ & KCNH1 \\
\hline ANK1 & other & & & $2,48 E-02$ & RHCERRD \\
\hline SERP1 & other & & & $2,48 E-02$ & Ins1 \\
\hline ARHGEF2 & other & & & $2,48 E-02$ & CCND1 \\
\hline TFP12 & other & & & $2,48 E-02$ & CCND1 \\
\hline PES1 & other & & & $2,48 E-02$ & CCND1 \\
\hline RBBP8 & enzyme & & & $2,48 E-02$ & CCND1 \\
\hline RABзB & enzyme & & & $2,48 E-02$ & SYP \\
\hline PDCD5 & other & & & $2,48 E-02$ & ATP2A2 \\
\hline CYP2C9 & enzyme & & & $2,48 E-02$ & CCND1 \\
\hline Irs4 & other & & & $2,48 E-02$ & $\operatorname{lns} 1$ \\
\hline CMYA5 & other & & & $2,48 E-02$ & ATP2A2 \\
\hline ATAD2 & enzyme & & & $2,48 E-02$ & CCND1 \\
\hline SB-657510 & chemical reagent & & & $2,48 E-02$ & ATP2A2 \\
\hline (E)-1-(2-nitroviny)|naphthalene & chemical reagent & & & $2,48 E-02$ & CCND1 \\
\hline (E)-9-(2-nitroviny)|anthracene & chemical reagent & & & $2,48 E-02$ & CCND1 \\
\hline Nethylmaleimide & chemical reagent & & & $2,48 E-02$ & GHR \\
\hline rocuronium & chemical drug & & & $2,48 E-02$ & CCND1 \\
\hline tarenflubbil & chemical drug & & & $2,48 E-02$ & CCND1 \\
\hline nitromifene & chemical reagent & & & $2,48 E-02$ & SYP \\
\hline betamethasone valerate & chemical drug & & & $2,48 E-02$ & CCND1 \\
\hline МK-1642 & chemical reagent & & & $2,48 E-02$ & LGALS12 \\
\hline phosphoramidon & hemical - endogenous non-manmalia & & & $2,48 E-02$ & ECE1 \\
\hline R-etodolac & chemical drug & & & $2,48 E-02$ & CCND1 \\
\hline cyclohexanecarboxylic acid & chemical reagent & & & $2,48 E-02$ & SYP \\
\hline mizoribine & chemical drug & & & $2,48 E-02$ & CCND1 \\
\hline ponasterone $A$ & chemical reagent & & & $2,48 E-02$ & CCND1 \\
\hline KLF4 & transcription regulator & $-1,334$ & bias & $2,51 E-02$ & ATP2A2, CCND1, DLL4,FRZB,PROM1,WLS \\
\hline
\end{tabular}




\begin{tabular}{|c|c|c|c|c|c|c|c|c|}
\hline F2 & & peptidase & & $-1,762$ & bias & $2,51 \mathrm{E}-02$ & CCND1,ECE1,GGBP5, ,RABBA,SYP,TRPC1 & \\
\hline MAP2K6 & & kinase & & & & $2,56 E-02$ & ATP2A2,CCND1,VDR & \\
\hline ZNF281 & & transcription regulator & & & & $2,58 E-02$ & PROM1,XRCC3 & \\
\hline HZAFZ & & other & & & & $2,58 \mathrm{E}-02$ & CCND1,IGFBP5 & \\
\hline MLXIPL & $-0,866$ & transcription regulator & & & & $2,58 \mathrm{E}-02$ & $A C L Y$, Ins1 & \\
\hline CXCL12 & & cytokine & & $-1,299$ & bias & $2,58 \mathrm{E}-02$ & BMP1,CCND1,GAD1,PDX1,XRCC3 & \\
\hline lactacystin & & chemical - protease inhibitor & & $-0,692$ & bias & $2,58 E-02$ & CCND1,GGFPF5,PDX1,PRLR,VDR & \\
\hline ATN1 & & transcription regulator & & & & $2,59 \mathrm{E}-02$ & BMP1,DBP,GAD1,IGFBP5 & \\
\hline dinoprost & & chemical- endogenous mammalian & & & & $2,65 E-02$ & ATP2A2, CCND1,PRLR & \\
\hline nifedipine & & chemical drug & & & & $2,65 \mathrm{E}-02$ & IGFBP5,Ins1,NTRK2 & \\
\hline MAPK11 & & kinase & & & & $2,74 E-02$ & INS,VDR & \\
\hline PRKAR1A & & kinase & & & & $2,74 E-02$ & CCND1,VDR & \\
\hline THBS4 & & other & & & & $2,74 E-02$ & MANF,SDF2L1 & \\
\hline Hmgna & & other & & & & $2,74 E-02$ & INs, Ins1 & \\
\hline 4-tert-octyphenol & & chemical toxicant & & & & $2,74 E-02$ & DLG3,Мuc4 & \\
\hline ROR2 & & kinase & & & & $2,74 E-02$ & CCND1,CDK5RAP1,SLC17A9 & \\
\hline GCG & & other & & & & $2,74 E-02$ & INS, Inst, PDX1 & \\
\hline CASR & & G-protetin coupled receptor & Inhibitited & $-2,000$ & bias & $2,78 E-02$ & CCND1,GFBP5,TLK1,VDR & \\
\hline haloffuginone & & chemical drug & & & & $2,78 E-02$ & CD200,MLXIPL,PRLR,PROM1 & \\
\hline n-nitrosomethylbenzylamine & & chemical toxicant & & & & $2,78 E-02$ & CCND1,GNG12,PCSK9,PECR & \\
\hline IGFIR & & transmembrane receptor & Inhibited & $-2,379$ & bias & $2,88 E-02$ & 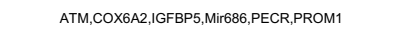 & \\
\hline kN 93 & & chemical - kinase inhibitior & & & & $2,90 \mathrm{E}-02$ & CCND1,GAD1 & \\
\hline RETNLB & & other & & & & $2,92 E-02$ & BMP1,FRZB,MID1 & \\
\hline EGF & & growth factor & & $-0,754$ & bias & $2,96 \mathrm{E}-02$ & ANPEP,ATM,ATP2A2,CCND1,HIRA,IGFBP5, Ins1,SYP,VDR & \\
\hline fluoxetine & & chemical drug & & & & $3,02 E-02$ & ADARB1,NTRK2,WIP11 & \\
\hline КDМзА & & transcription regulator & & & & $3,08 E-02$ & CCND1,Ins1 & \\
\hline imipramine blue & & chemical drug & & & & $3,08 E-02$ & CCND1,XRCC3 & \\
\hline Calcineurin A & & group & & & & $3,25 E-02$ & ATP2A2,CCND1 & \\
\hline sox17 & & transcription regulator & & & & $3,25 \mathrm{E}-02$ & CCND1,PROM1 & \\
\hline TFDP1 & & transcription regulator & & & & $3,25 \mathrm{E}-02$ & ATM,CCND1 & \\
\hline CGP 74514A & & chemical - kinase inhibitor & & & & $3,29 \mathrm{E}-02$ & CCND1 & \\
\hline L-alanine & & chemical-endogenous mammalian & & & & $3,29 E-02$ & CCND1 & \\
\hline ribose & & chemical- endogenous mammalian & & & & 3,29E-02 & Ins1 & \\
\hline inecalcitiol & & chemical drug & & & & $3,29 E-02$ & CCND1 & \\
\hline HCFC1 & & transcription regulator & & & & $3,29 E-02$ & PDX1 & \\
\hline AKAP5 & & other & & & & $3,29 \mathrm{E}-02$ & Ins1 & \\
\hline aloe-emodin & & chemical toxicant & & & & $3,29 \mathrm{E}-02$ & ATM & \\
\hline
\end{tabular}




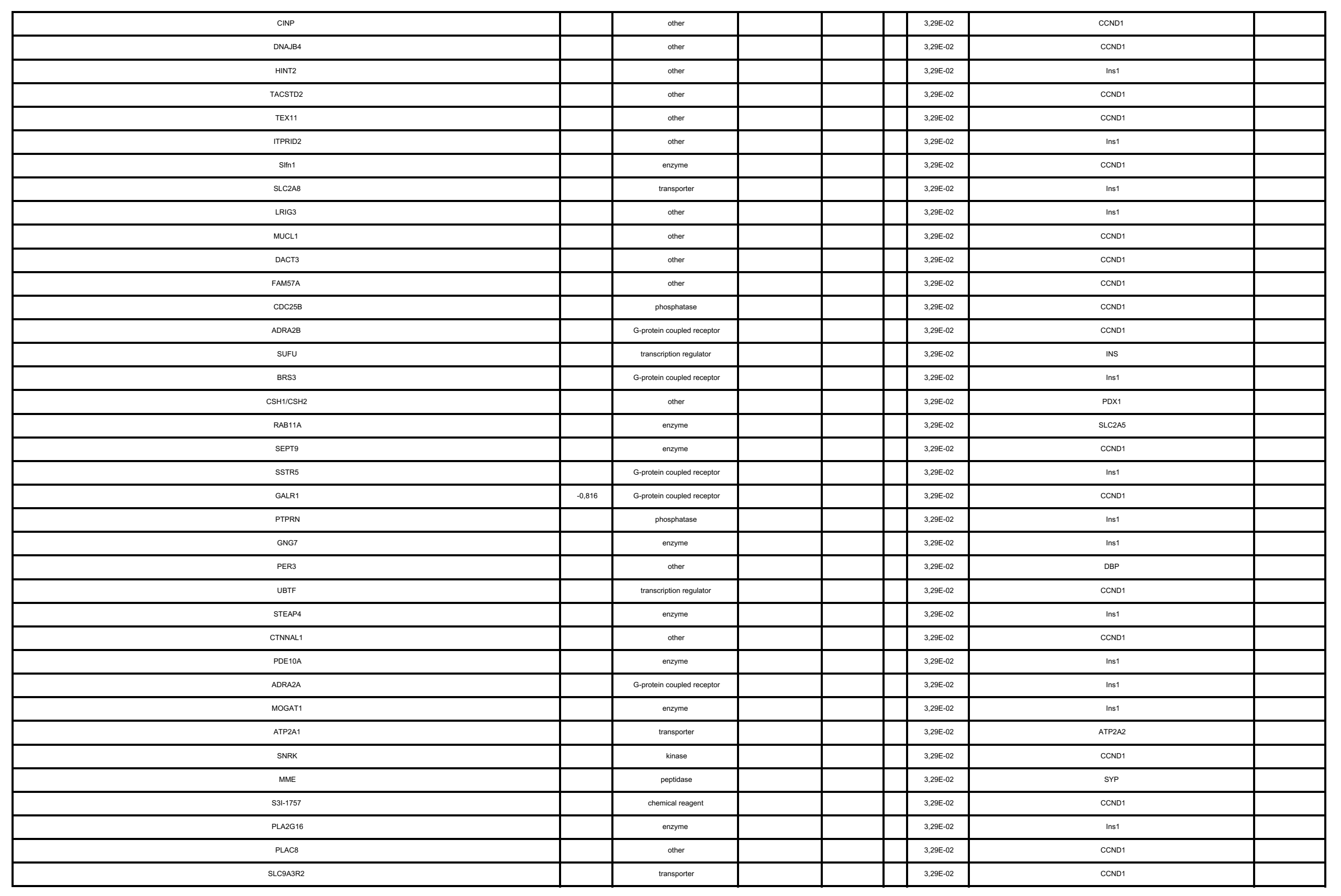




\begin{tabular}{|c|c|c|c|c|c|c|c|}
\hline ARF1 & enzyme & & & & $3,29 \mathrm{E}-02$ & CCND1 & \\
\hline PMX 205 & chemical reagent & & & & 3,29E-02 & BMP1 & \\
\hline LY5 & chemical reagent & & & & $3,29 \mathrm{E}-02$ & CCND1 & \\
\hline phenylacetic acid & chemical-endogenous mammalian & & & & $3,29 \mathrm{E}-02$ & Ins1 & \\
\hline carboxyamido-triazole & chemical drug & & & & 3,29E-02 & CCND1 & \\
\hline acetaacetic acid & chemical- endogenous mammalian & & & & 3,29E-02 & CCND1 & \\
\hline KP-SD-1 & chemical - kinase inhibitor & & & & 3,29E- -02 & CCND1 & \\
\hline liarozole & chemical drug & & & & $3,29 E-02$ & VDR & \\
\hline phosphorus & chemical reagent & & & & 3,29E- -22 & Ins1 & \\
\hline CDP-choline & chemical- endogenous mammalian & & & & $3,29 \mathrm{E}-02$ & SYP & \\
\hline vigabatrin & chemical drug & & & & $3,29 \mathrm{E}-02$ & GAD1 & \\
\hline L-asparagine & chemical-endogenous mammalian & & & & $3,29 \mathrm{E}-02$ & CCND1 & \\
\hline Collagen type VI & complex & & & & 3,29E-02 & CCND1 & \\
\hline STATSB & transcription regulator & & 0,211 & & $3,29 E-02$ & ATM,ATP2A2,CCND1,COX6A2,PRLR,PROM1 & \\
\hline sildenafil & chemical drug & & & & 3,43E-02 & SYP,TRPC1 & \\
\hline sodium orthovanadate & chemical reagent & & & & 3,43E- -02 & CCND1,SLC2A5 & \\
\hline exenatide & biologic drug & & & & 3,43E- -02 & GLP1R,PDX1 & \\
\hline estrogen & chemical drug & Inhibited & $-2,371$ & bias & 3,44E-02 & 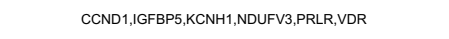 & \\
\hline РКз3R1 & kinase & & & & 3,52E-02 & CCND1,Ins1,PDX1 & \\
\hline LDLR & transporter & & & & $3,55 E-02$ & ACLY,CCND1,ns1,NOD1,PKIB & \\
\hline carbon tetrachloride & chemical toxicant & & & & $3,55 \mathrm{E}-02$ & FAAH,IGFBP5,Ins 1,PDX1,PROM1 & \\
\hline methylprednisolone & chemical drug & & 0,563 & & $3,56 E-02$ & ANPEP,ATP2A2,CCND1,ECE1,FAAH,GHR,MLXIPL,NDUFV3,SCLY & \\
\hline 8-bromoguanosine 3;'5-cyclic monophosphate & chemical - kninase inhibitor & & & & $3,61 \mathrm{E}-02$ & CDK14,PCCA & \\
\hline GTF2B & transcription regulator & & & & $3,61 E-02$ & GHR,PRLR & \\
\hline amiodarone & chemical drug & & & & $3,61 \mathrm{E}-02$ & SLCO2B 1,WIPI1 & \\
\hline ISL1 & transcription regulator & & & & $3,62 E-02$ & ETV5,Ins1,NTRK2 & \\
\hline ERBB4 & kinase & & & & $3,62 E-02$ & CCND1,GHR,IGFBP5 & \\
\hline HDL-cholesterol & complex & & & & 3,80E-02 & ALDH1L2,TRIM66 & \\
\hline SERPINE1 & other & & & & $3,80 \mathrm{E}-02$ & Ins1,SYP & \\
\hline North & group & & 0,152 & bias & 3,93E-02 & ACLY,ECE1,MLXIPL,PDX1 & \\
\hline TCF & group & & & & 4,05E-02 & CCND1,GAD1,PCCA & \\
\hline 1.2-2-dioctanoyl-sn-gycerol & chemical reagent & & & & $4,10 \mathrm{E}-02$ & VDR & \\
\hline L-aspartic acid & chemical- endogenous mammalian & & & & $4,10 E-02$ & CCND1 & \\
\hline p38 Sapk & group & & & & $4,10 \mathrm{E}-02$ & CCND1 & \\
\hline ILX-23-7553 & chemical drug & & & & $4,10 \mathrm{E}-02$ & CCND1 & \\
\hline TPPP2 & other & & & & $4,10 \mathrm{E}-02$ & Атм & \\
\hline liraglutide & biologic drug & & & & $4,10 \mathrm{E}-02$ & GLP1R & \\
\hline
\end{tabular}




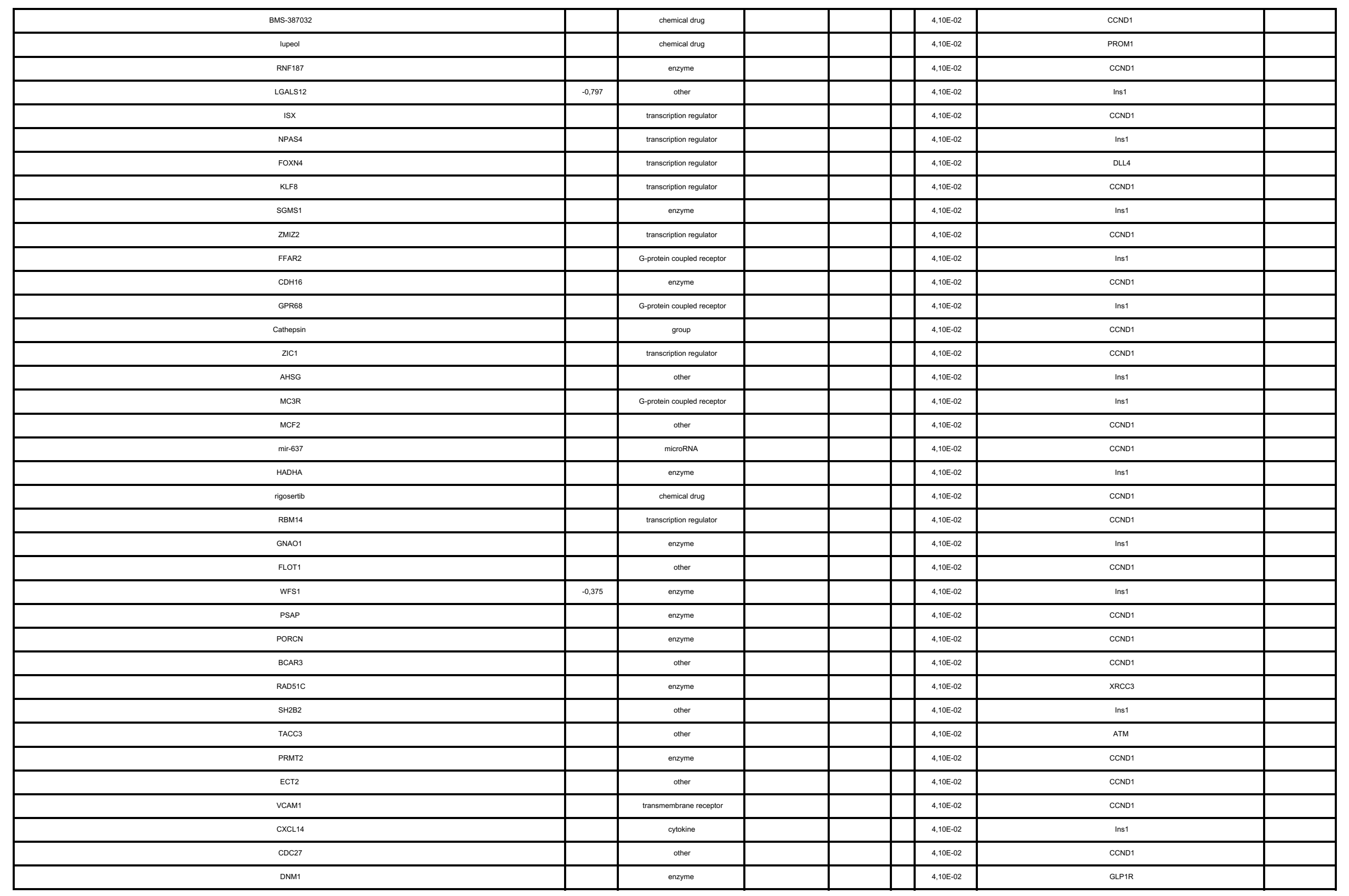




\begin{tabular}{|c|c|c|c|c|c|c|}
\hline SPRY4 & other & & & $4,10 \mathrm{E}-02$ & ETV5 & \\
\hline KRT10 & other & & & $4,10 \mathrm{E}-02$ & CCND1 & \\
\hline Muct & transmembrane receptor & & & $4,10 \mathrm{E}-02$ & CCND1 & \\
\hline кмт2в & transcription regulator & & & $4,10 E-02$ & Ins1 & \\
\hline Grs2 & enzyme & & & $4,10 E-02$ & MLXIPL & \\
\hline CLDN2 & other & & & $4,10 \mathrm{E}-02$ & CCND1 & \\
\hline scoparone & hremical - endogenous non-manmmalia & & & $4,10 E-02$ & CCND1 & \\
\hline silicon phthalocyanine & chemical drug & & & $4,10 E-02$ & CCND1 & \\
\hline temsirolimus & chemical drug & & & $4,10 \mathrm{E}-02$ & CCND1 & \\
\hline 2,2-2-dipyridyl & chemical toxicant & & & $4,10 \mathrm{E}-02$ & NTRK2 & \\
\hline atenolal & chemical drug & & & $4,10 \mathrm{E}-02$ & ATP2A2 & \\
\hline nigericin & chemical drug & & & $4,10 \mathrm{E}-02$ & PROM1 & \\
\hline ASCL1 & transcripition regulator & & & $4,16 \mathrm{E}-02$ & DLL4,GAD1,INS & \\
\hline SLC13A1 & transporter & & & $4,16 \mathrm{E}-02$ & ACLY,SDF2L1,SRPRA & \\
\hline GW9662 & chemical reagent & & & $4,16 \mathrm{E}-02$ & ACLY,FFAR1,PDX1 & \\
\hline kainic acid & chemical toxicant & & & $4,17 E-02$ & CCND1,CD200,NTRK2,SYP & \\
\hline carbamylcholine & chemical drug & & & $4,38 E-02$ & CCND1,ECE1 & \\
\hline propranolol & chemical drug & & & $4,38 E-02$ & ATP2A2,LGALS12 & \\
\hline PDLIM2 & other & & & $4,39 E-02$ & CCND1,FHDC1,GHR & \\
\hline ERK $1 / 2$ & group & $-1,067$ & bias & $4,40 E-02$ & CCND1,ECE1,GAD1,INS Ins1,PTPN11 & \\
\hline ibuprofen & chemical drug & & & $4,58 \mathrm{E}-02$ & CCND1,TNFRSF21 & \\
\hline $\mathrm{H}-\mathrm{-}$ & chemical - kinase inhibitor & & & $4,58 \mathrm{E}-02$ & ECE1,VDR & \\
\hline ATF4 & transcription regulator & & & $4,59 E-02$ & IGFBP5, Ins, PYCR1,WFS1 & \\
\hline SLC25A13 & transporter & & & $4,62 E-02$ & ACLY,CCND1, Ins1 & \\
\hline IL10RA & transmembrane receptor & $-1,633$ & & $4,69 E-02$ & ADH1C,GHR,LGALL12,NOD1,SLCO2B1,SORBS2 & \\
\hline wT1 & transcription regulator & $-1,160$ & & $4,72 E-02$ & ANPEP,CCND1,LMAN1,NTRK2,VDR & \\
\hline olanzapine & chemical drug & & & 4,74E-02 & DBP,GALR1,INS & \\
\hline PC.SPES & chemical drug & & & $4,74 E-02$ & ACLY,CCND1,IMPDH1 & \\
\hline Laminin (complex) & complex & & & $4,78 E-02$ & CCND1,ETV5 & \\
\hline KL & enzyme & & & $4,78 E-02$ & CCND1,VDR & \\
\hline PKM & kinase & & & $4,78 E-02$ & ACLY,CCND 1 & \\
\hline PTEN & phosphatase & $-1,417$ & & $4,84 E-02$ & 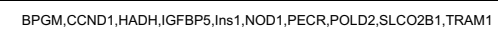 & \\
\hline UCP1 & transporter & 1,000 & & 4,85E-02 & EML1,ENHO, Ins1,PYCR1 & \\
\hline LGALS3 & other & & & $4,866-02$ & CCND1,GFBP5,LGALS12 & \\
\hline IGF1 & growhth factor & $-1,473$ & bias & $4,87 \mathrm{E}-02$ & ANPEP,ATM,CCND1,GHR,IGFBP5, InS1,NTRK2,PRLR & \\
\hline prednisone & chemical drug & & & $4,89 \mathrm{E}-02$ & INS & \\
\hline varilly-N-nonylamide & chemical drug & & & 4,89E-02 & CCND1 & \\
\hline
\end{tabular}




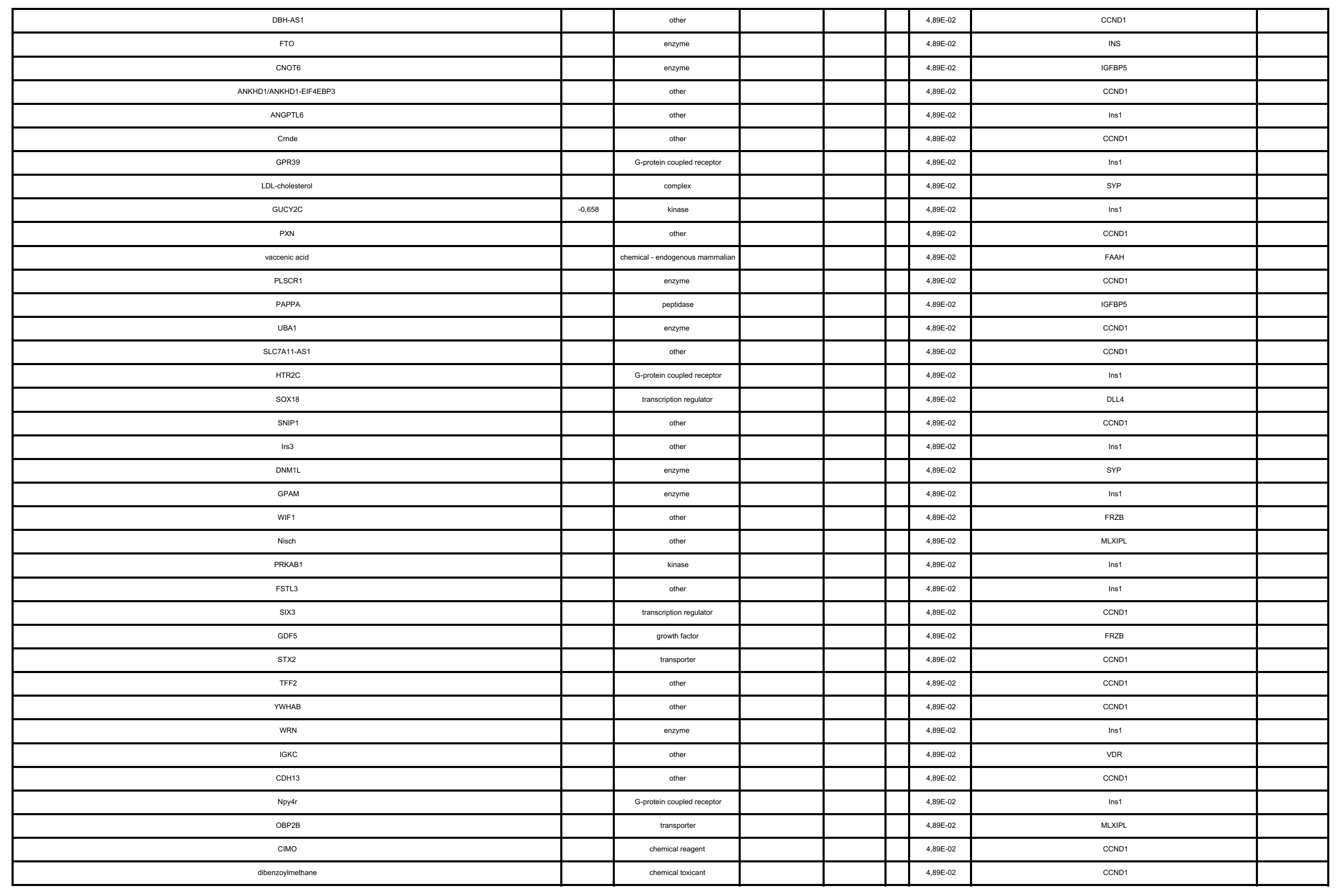




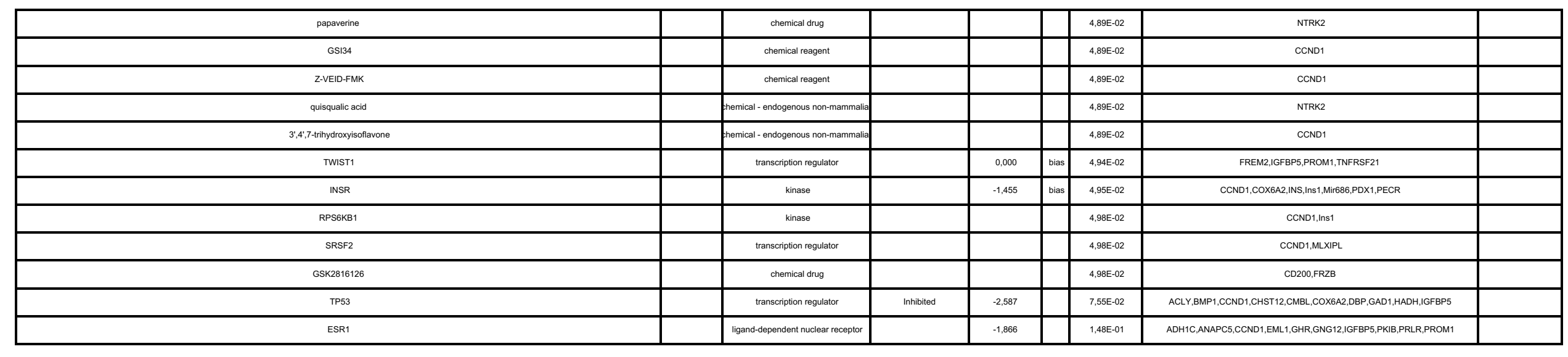




\begin{tabular}{|c|c|c|c|c|c|c|c|c|}
\hline Upstream Regulator & Expr Log Ratio] & Molecule Type & Predicted Activation Statatg & |Activation z-score & Flags & p-value of overlap & Target Molecules in Dataset & Mechanistic Network \\
\hline TNF & & cytokine & Activated & 6,542 & bias & $9,24 E-35$ & A4GALT,ACOD1,AKR11810,ALCAM,,ALDH2,AMPD3,APOA1,APOBECBB,ARIID5B,B44GLLNT1 & $192(14)$ \\
\hline IFNG & & cytokine & Activated & 6,621 & bias & $7,25 E-33$ & ACOD1,BCL2A1,BIRC3,BLNK,BMF,BMPG,BST1,CIR,CAMK4,CASP3 & $199(18)$ \\
\hline lipopolysaccharide & & chemical drug & Activated & 8,302 & bias & 3,99E-31 & ACOD1,ALCAM,ALDH2,AMPD3,ANXA3,APOA1,APOBEC $3 B$,Apol7e (includes others),ARHGEF3,BATF & $215(14)$ \\
\hline TGFB1 & & growth factor & Activated & 3,805 & bias & $1,75 E-23$ & ADAM19,ALDH2,ARIDSB,BMF,BMP2,BMP4,BMPG,BUB1,C1R,CASP3 & $235(19)$ \\
\hline dexamethasone & & chemical drug & & 1,059 & & $6,77 E-23$ & ACOT7,ALAS1,ALCAM,AMIGO2,AMPD3,ANXA3,APOA1,BATF,BCLLA1,BRC3 & $222(15)$ \\
\hline แ18 & 1,038 & cytokine & Activated & 5,905 & bias & $1,30 \mathrm{E}-22$ & A4GALL,ACOD1,AMPD3,ANTXR1,BCL2A1,BRC3,BMF,BMP2,BMPA,C1R & $240(19)$ \\
\hline NFKBBA & 0,623 & transcription regulator & Activated & 2,801 & bias & $9,07 E-19$ & ACOD1,AMPD3,APOA1,BCL11A,BCL2A1,BIRC3,BMP2,CCNA2,CD40,CD86 & $196(17)$ \\
\hline TNFSF11 & & cytokine & Activated & 5,699 & bias & $2,84 E-18$ & ACOD1,BCL2A1,BMP4,BST1,CD14,CD40,CD44,CD83,СEBPA,CSF3 & $133(14)$ \\
\hline beta-estradiol & & chemical-endogenous manmmalian & Activated & 2,051 & bias & $3,85 E-17$ & A4GALT,ALCAM,,ANXA3,APOA1,ARIID5B,BATF,BCLLA1,BIRC3,BLNK,BMP2 & $263(21)$ \\
\hline CSF2 & & cytokine & Activated & 5,651 & bias & 4,93E-17 & 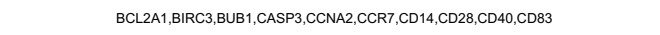 & $232(17)$ \\
\hline IL4 & & cytokine & Activated & 3,078 & bias & $5,78 E-17$ & ACOD1,ADAM19,ALAS1,ALDH2,ANXAG,B4GALLT1,BCL11A,CAMK1D,CASP3,CCR7 & $187(16)$ \\
\hline IKZF1 & & transcription regulator & Inhibited & $-4,354$ & bias & $2,66 E-16$ & 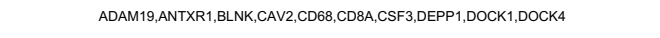 & \\
\hline trichostatin A & & chemical drug & Activated & 2,202 & & $7,12 E-16$ & 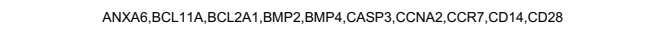 & 229 (22) \\
\hline MYD88 & & other & Activated & 4,953 & bias & $1,59 \mathrm{E}-15$ & 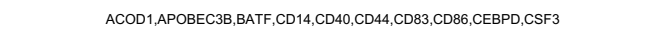 & $192(14)$ \\
\hline HRAS & & enzyme & & $-0,331$ & bias & $1,75 E-15$ & ANTXR1,ANXA3,ANXAG, BIRC3,BMP4,CCNA2, СD44,CD81,CEBPD,CRIM1 & $223(19)$ \\
\hline NFkB (complex) & & complex & Activated & 6,136 & bias & $3,04 E-15$ & B4GALT1,BATF,BCL11A,BCLLA1,BRCC3,BMP2,C1R,CCKAR,CCR7,CD40 & $185(12)$ \\
\hline decitabine & & chemical drug & Activated & 3,610 & bias & $6,35 E-15$ & BCL11A,BRC3,BMP4,BTC3,,CCNA2,CCR7,CD44,CDBA,CD9,,CDCA7 & $219(20)$ \\
\hline ІКвкв & & kinase & Activated & 3,454 & bias & $7,53 E-15$ & ACOD1,AMPD3,BIRC3,BMP2,CCNA2,CCR7,CD44,CEBPD,CP,CSF1 & $185(13)$ \\
\hline cyclosporin A & & biologic drug & & 0,308 & bias & 9,42E-15 & ALAS1,ANG,BATF,BRC3,BMP2,CASP3,CCR7,CD14,CD40,CD44 & $214(21)$ \\
\hline แ10 & & cytokine & & 0,825 & & $1,16 \mathrm{E}-14$ & BCL2A1,BMP2,BMP, ,ASP 3,CCR7,CD14,CD2,CD28,CD40,CD44 & $180(19)$ \\
\hline TICAM1 & & other & Activated & 4,566 & bias & $2,89 E-14$ & ACOD1,APOBEC3B,Apol7e (includes others),CC12,CD40,CD86,CEEPD,FAS,GDF15,ICAM1 & $137(17)$ \\
\hline IL2 & & cytokine & Activated & 5,046 & bias & 3,44E-14 & 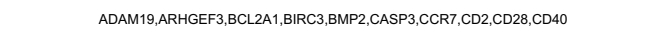 & $180(19)$ \\
\hline IL6 & & cytokine & Activated & 4,595 & bias & $1,19 E-13$ & AKR1B10,ALAS1,ANG,APOA1,BATF,BMP2,BMPG,BUB1, СASP3, CCNA2 2 & $191(16)$ \\
\hline OGA & & enzyme & & $-0,434$ & & $1,51 E-13$ & ADAM19,AHNAK2,CAMK1D, CD144,CD40,CD44,CD68, CD86,,CDCA7,,CEBPA & $114(7)$ \\
\hline cisplatin & & chemical drug & Activated & 4,037 & bias & $1,69 \mathrm{E}-13$ & AHNAK2,BCL2AA, BIRC3,BMP4,CASP3,CD 14,CD44,CD68,CEBPA,CLCA1 & $219(20)$ \\
\hline triamterene & & chemical drug & Activated & 3,965 & bias & 2,95E-13 & BIRC3,CD 14,CD 44, CLDN4,CP,LAMC2,MCM3,MRC1,PSMB10,RCN1 & \\
\hline poly li:lC-RNA & & biologic drug & Activated & 5,531 & bias & 5,77E-13 & ACOD1,Apol7e (includes others),BRC3,,BMP2,CCR7,CD14,CD40, СD44,CD83, CD86 & $194(14)$ \\
\hline fenamic acid & & chemical reagent & Activated & 3,959 & bias & 6,24E-13 & BIRC3,СD 14,CD 44, СLDN4,CP,LAMC2,MCM3,MRC1,PSMB10,RCN1 & \\
\hline salmonella minnesota R595 lipopolysacccharides & & hemical - endogenous non-mammalia & Activated & 4,378 & bias & $7,27 E-13$ & ACOD1,CC12,CC18,CD83,CD866,CEBPD,FOSL1,,GDF15,ICAM1,L1B & $182(16)$ \\
\hline Immunoglobulin & & complex & & 0,524 & & $8,30 E-13$ & ADAM19,BATF,BCL2A1,BIRC3,CCR7,CD 14,CD28,CD40,CD44,CD68 & $172(17)$ \\
\hline СЕВРВ & & transcription regulator & Activated & 4,177 & bias & $9,07 E-13$ & AKR1B10,ANTXR1,BCL2A1,BLNK,CCNA2,CD14,CEBPA,CEBPD,,CP,CSF3 & $204(20)$ \\
\hline ІКвкG & & kinase & Activated & 3,290 & bias & $1,03 E-12$ & ACOD1,AMPD3,BIRC3,BMP2,CCI2,CEBPD,CP,CSF1,CTSZ,CXCL12 & $209(13)$ \\
\hline allopurinol & & chemical drug & Activated & 3,637 & bias & $1,56 \mathrm{E}-12$ & BIRC3,CD14,CD44,CLDN4,CP, IL18,LAMC2,MCM3,MRC1,PSMB10 & \\
\hline vancomycin & & biologic drug & Activated & 3,483 & bias & $2,84 E-12$ & BIRC3,СD14,CD44,,LDN4,CP,LAMC2,MCM3,MRC1,PSMB 10,RCN1 & \\
\hline STAT3 & & transcription regulator & & 1,803 & bias & 3,79E-12 & 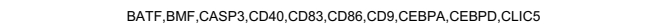 & $185(22)$ \\
\hline
\end{tabular}




\begin{tabular}{|c|c|c|c|c|c|c|c|c|}
\hline KRAS & & enzyme & & 1,188 & & $3,94 \mathrm{E}-12$ & 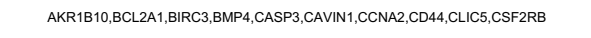 & $203(21)$ \\
\hline "LA & & cytokine & Activated & 4,591 & bias & $4,06 E-12$ & 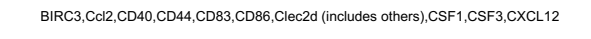 & $180(15)$ \\
\hline resiquimod & & chemical drug & Activated & 3,556 & bias & $4,58 \mathrm{E}-12$ & 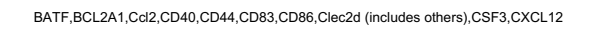 & $145(16)$ \\
\hline puromycin aminonucleoside & & chemical reagent & Activated & 2,621 & bias & $4,61 \mathrm{E}-12$ & CD14,CD44,CLDN4,CLIC5,CP, СYBA,,GSTP1,LAMC2,MCM3,MRC1 & \\
\hline progesterone & & chemical - endogenous mammalian & & $-0,054$ & & $5,14 \mathrm{E}-12$ & 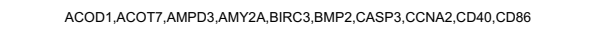 & $218(18)$ \\
\hline doxorubicicin & & chemical drug & Activated & 4,451 & bias & $7,23 \mathrm{E}-12$ & ANXA6,BCL2A $1, B 1 R C 3, B M P 4, C A S P 3, C D 144, C D 40, C D 44, C L D N 4, C P$ & $215(19)$ \\
\hline E. coli B5 lipopolysaccharide & & hemical- endogenous non-mammalia & Activated & 4,073 & bias & $7,64 \mathrm{E}-12$ & ALDH2,APOA1,BRC3,CCR7,CD40,СD44,CD83,СD86,СEBPA,CSF3 & $176(14)$ \\
\hline «233 & & cytokine & Activated & 4,282 & bias & $8,75 E-12$ & 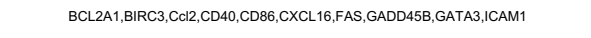 & $146(16)$ \\
\hline EP300 & & transcription regulator & & 0,647 & bias & $1,07 E-11$ & 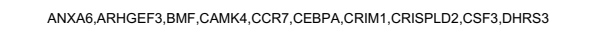 & $248(23)$ \\
\hline PAARG & & ligand-dependent nuclear receptor & & 0,760 & bias & 1,41E-11 & ACOD1,APOA1,CAV2,,CL12,CCR7,,CD40,CEBPA,CSF3,CXCL14,CYBA & $232(21)$ \\
\hline снUк & & kinase & Activated & 4,203 & bias & $1,69 \mathrm{E}-11$ & 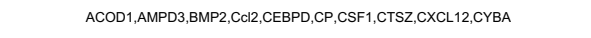 & $192(14)$ \\
\hline ZвтB16 & 3,395 & transcription regulator & & 0,209 & & $1,77 \mathrm{E}-11$ & 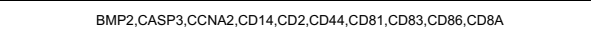 & $160(7)$ \\
\hline TP53 & & transcription regulator & Activated & 4,025 & & $2,85 E-11$ & 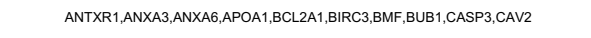 & $229(22)$ \\
\hline tretinoin & & chemical-endogenous mammalian & Activated & 4,606 & bias & $2,89 E-11$ & 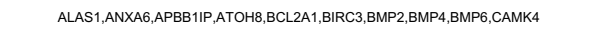 & $213(21)$ \\
\hline cG & & complex & Activated & 4,388 & bias & $2,94 \mathrm{E}-11$ & AKR1110,BMP2,BTG3,CAMK4,CCL2, CEBPA,CEBPD,DMD,FABP5,FAS & $238(22)$ \\
\hline STAT1 & & transcription regulator & Activated & 4,251 & bias & $3,62 E-11$ & ACOD1,ALPK1,APOBECBB,C1R,CASP3,,CCR7,CD 14,CD40,CD86,,СEBPD & $191(18)$ \\
\hline gentamicin C & & chemical drug & Activated & 3,729 & bias & $4,12 E-11$ & BIRC3,CD14,CD44,CLDN4,CP,DMD,LAMC2,MCM3,MRC1,PSMB10 & \\
\hline lomustine & & chemical drug & Activated & 3,742 & bias & $4,12 E-11$ & BIRC3,СD14,CD44,CLNN4,CP,LAMC2,MCM3,RCN1,SLC34A2,TMEM173 & \\
\hline ERK $1 / 2$ & & group & Activated & 3,040 & bias & $6,30 \mathrm{E}-11$ & 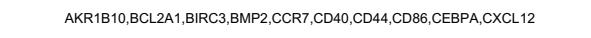 & $215(17)$ \\
\hline Alpha catenin & & group & Inhibited & $-4,059$ & bias & $6,41 E-11$ & 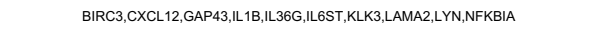 & $177(15)$ \\
\hline STATSA & & transcription regulator & & 0,745 & & $7,46 E-11$ & 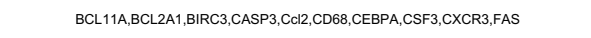 & 252 (19) \\
\hline CD40LG & & cytokine & Activated & 3,119 & bias & $8,19 E-11$ & A4GALL,ALCAM,ANXAG,BATF,BCLZA1, BIRC33,BTG3,CCR7,CD40,CD44 & $138(14)$ \\
\hline ILORA & & transmembrane receptor & & $-1,612$ & & $9,20 E-11$ & ACOD1,ALDH2,BMP2,BMP4,CCR7,,C224, CD40,FABP5, FADS3,FAS & 158 (13) \\
\hline 102 & & transcription regulator & & 0,786 & & 9,43E-11 & BATF,CCR7,CD44,CD83,CSF1,CSF2RB,CXCR3,GADD45B,HOMER2,ICAM1 & $135(7)$ \\
\hline prostaglandin E2 & & chemical-endogenous mammalian & Activated & 4,183 & bias & $1,07 E-10$ & 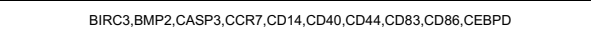 & $150(16)$ \\
\hline TCF3 & & transcription regulator & & 1,213 & bias & $1,08 E-10$ & ALCAM,ANXAG,BLNא,BUB1,CASP3, CCNA2, CD2, Cliez2d (includes others), CTRB2, DOCK1 & $30(2)$ \\
\hline ERBB2 & & kinase & Activated & 2,690 & bias & $1,08 E-10$ & ADAM19,ALAA1,ANG,BIRC3,BUB1,CAVII1,CCNA2,CD9, CDCA7,CLDN4 & $230(23)$ \\
\hline mifepristone & & chemical drug & & $-1,001$ & & $1,10 \mathrm{E}-10$ & 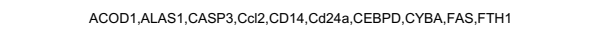 & 254 (23) \\
\hline PIзK (complex) & & complex & Activated & 3,094 & bias & $1,29 E-10$ & BIRC3,СASP3,CC12,CD2,CD44,CD86,FABP5,FOSL1, GAP43,GATA3 & $203(19)$ \\
\hline Jnk & & group & Activated & 3,528 & bias & $1,56 E-10$ & 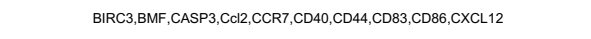 & $180(16)$ \\
\hline methylprednisolone & & chemical drug & & 1,908 & & 1,75E-10 & ALAS1,ANXA3,84GALINT1,Cald1,CAMK1D,CC12,CD24a, CD44,CD9,DMD & $215(20)$ \\
\hline ID3 & 0,768 & transcription regulator & & 0,549 & & $1,90 \mathrm{E}-10$ & BATF,CCR7,CD44,CD83,CSF1,CSF2RB,CXCR3,,GADD458,HOMER2,ICAM1 & $174(11)$ \\
\hline VCAN & & other & & $-1,309$ & & $1,98 E-10$ & 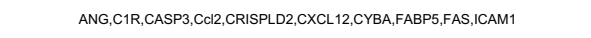 & 161 (19) \\
\hline 2-bromoethylamine & & chemical reagent & Activated & 3,434 & bias & $2,51 \mathrm{E}-10$ & CD 14,CD44,CLDN4,CP,LLAMC2,MCM3,MRC1,SLC34A2,TMEM173,TNFRSF 12 A & \\
\hline E. coli B4 ilipopolysaccharide & & chemical toxicant & Activated & 3,764 & bias & $2,62 E-10$ & ACOD1,AMPD3,СASP3,CC12,CCR7,CD40,CD83,CD866,CSF3,FAS & $183(17)$ \\
\hline ethionine & & chemical toxicant & Activated & 2,720 & bias & $2,67 \mathrm{E}-10$ & CD14,CD44,CP,LAMC2,MCM3,PSMB 10,SLC34A2,TNFRSF 12A,TPm1,TSPAN8 & \\
\hline SP1 & & transcription regulator & Activated & 2,869 & bias & $2,68 E-10$ & APOA $1, B M P 4, C A P P 3, C C 12, C C N A 2, C D 28, C D 40, C E B P A, C E B P D,, C X C L 12$ & $202(21)$ \\
\hline
\end{tabular}




\begin{tabular}{|c|c|c|c|c|c|c|c|c|}
\hline CD40 & 0,881 & transmembrane receptor & Activated & 3,352 & bias & $2,70 \mathrm{E}-10$ & A4GALT,BCLLA1,BIRC3,CCNA2,CD40,CD44,CD83,CD86,,CD8A,CSF1 & $155(15)$ \\
\hline Мүв & & transcription regulator & & $-1,340$ & bias & $3,07 E-10$ & BIRC3,BLNK,BMF,CC12,CD14,CSF3,CXCL12,CXCL16,,GATA3,ICAM1 & $195(20)$ \\
\hline ESR1 & & ligand-dependent nuclear receptor & & 0,647 & & $3,26 E-10$ & ALCAM,ANXA3,APOA1,BIRC3,BMP2,BMP4,BUB1,CAVV2,CBLC,CCNA2 & $234(20)$ \\
\hline NOTCH1 & & transcription regulator & Activated & 2,581 & & $3,34 E-10$ & 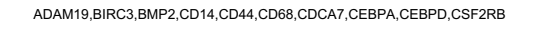 & $186(15)$ \\
\hline STATG & & transcription regulator & & 0,107 & & $3,40 E-10$ & ADAM19,ALDH2,CAMK1D,CC12,CCR7,CD2,CD244,CD40,DOCK10,EFHD2 & $175(16)$ \\
\hline SMARCA4 & & transcription regulator & Activated & 3,591 & bias & $3,78 E-10$ & 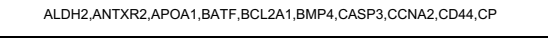 & $228(20)$ \\
\hline SP11 & & transcription regulator & Activated & 2,737 & bias & $3,91 E-10$ & BCL11A,BLNK,CCNA2,CCR7,CD14,CD68, CEBPA,CSFF1,CSF2RB,,FTH1 & $176(20)$ \\
\hline Interferon alpha & & group & Activated & 3,825 & bias & $3,98 E-10$ & A4GALT,ADAM19,APOBEC3B,BCL2A1,BMP4,CASP3,CCR7,CD40,СD81,СD83 & $172(17)$ \\
\hline BCL6 & & transcription regulator & & $-1,468$ & bias & $6,44 E-10$ & ALCAM,ANXAG,BCLLA1, BLNא,CCL2, CCR7,CD2, CD44,CSF1,DOCK10 & $167(18)$ \\
\hline ц.3 & & cytokine & Activated & 3,459 & bias & $7,60 \mathrm{E}-10$ & ALDH2,BIRC3,BMP2,CASP3, CD 14,CD40, CD833,CD86,,CSF1,CSF3 & $156(17)$ \\
\hline bleomycin & & chemical drug & Activated & 4,260 & bias & $8,81 E-10$ & BMP4,CASP3, СC12,CC18,CD68,CD86,CEBPA,CXCL 12,FAS,FOSL1 & 233 (19) \\
\hline ADRB & & group & Inhibited & $-3,154$ & bias & $9,41 E-10$ & 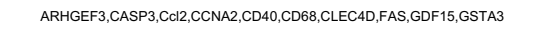 & $209(22)$ \\
\hline CREBBP & & transcription regulator & & 0,239 & bias & $9,93 E-10$ & ALAS1,ANXAG,ARHGEF 3, CAMK 4, CCR7,CD44, CEBPA,CRIM1,CRISPLD2,CSF1 & $193(18)$ \\
\hline tetradecanoyphorbol acetate & & chemical drug & Activated & 4,839 & bias & 1,01E-09 & 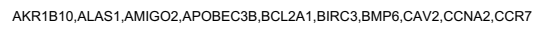 & $235(19)$ \\
\hline NR3C1 & & ligand-dependent nuclear recepptor & & $-0,977$ & & $1,05 E-09$ & 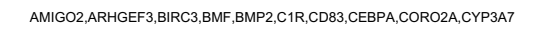 & $238(21)$ \\
\hline ATF3 & & transcription regulator & Inhibited & $-2,496$ & & $1,25 E-09$ & 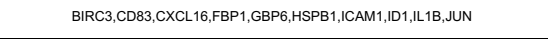 & $224(19)$ \\
\hline แ13 & & cytokine & Activated & 3,073 & bias & $1,26 \mathrm{E}-09$ & 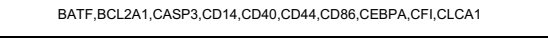 & $178(20)$ \\
\hline TCR & & complex & Activated & 2,535 & bias & 1,33E-09 & BATF,BCL2A1,BRRC3,CCR7,CD28,CD44,CD6,CXCR3,FAs,,GADD45B & $170(20)$ \\
\hline KLF2 & & transcription regulator & & $-1,899$ & & $1,42 E-09$ & BMP4,CC12,CCR7,CD40, СD44,CD866,CEBPA,CXCR3, ID 1,1D3 & $194(19)$ \\
\hline APOE & & transporter & & $-1,448$ & bias & 1,46E-09 & 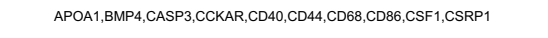 & $156(16)$ \\
\hline CPG oligonucleotide & & chemical drug & Activated & 3,749 & bias & $1,48 \mathrm{E}-09$ & BCL2A1,CD 14,CD40,CD83, CD86, CLEC4D,FAS,GBPF, GIycam1,ICAM1 & $180(22)$ \\
\hline JUN & 0,556 & transcription regulator & Activated & 3,524 & bias & 1,49E-09 & ALAS1,BCLLA1,BIRC3, CC12,CCNA2,CD14,CD44,CD68, CSF1,CXCL16 & 233 (23) \\
\hline rosigilizazone & & chemical drug & Activated & 2,010 & bias & $1,49 E-09$ & 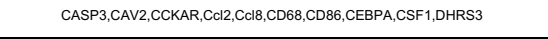 & $207(21)$ \\
\hline PDGF BB & & complex & Activated & 3,317 & bias & $1,56 E-09$ & 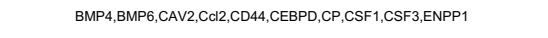 & $216(22)$ \\
\hline LY294002 & & chemical -kinase inhibitior & Inhibited & $-3,867$ & bias & 1,62E-09 & BIRC3,BMP2,CASP3,CCNA2,CD14,CD44,CD83,CD86,CEBPA,CLDN4 & $198(16)$ \\
\hline hexachlorobenzzene & & chemical toxicant & Activated & 3,464 & bias & $1,65 E-09$ & CD14, CD44,CP, LAMC2,MRC1,PSMB 10,RCN1, SLC34A2,,TNFRSF 12A, TSPAN8 & \\
\hline lge & & complex & Activated & 4,097 & bias & $1,78 E-09$ & 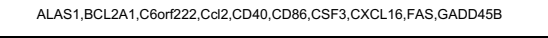 & $155(17)$ \\
\hline APP & & other & & 1,618 & bias & $1,83 E-09$ & ADAM19,ANXAG,BASP1,BMF,BMP4,BMPG,C1R,CASP3,CD40,CD44 & $220(17)$ \\
\hline GF11 & & transcription regulator & Inhibited & $-3,260$ & bias & $1,85 E-09$ & 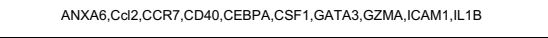 & $134(13)$ \\
\hline camptothecin & & chemical drug & & 1,879 & bias & 1,89E-09 & BCL11A,BCL2A1,BRC3,BTC3,СASP3,CD40,CD83,,CD86,CSF1,FAS & $170(15)$ \\
\hline TLR4 & & transmembrane receptor & Activated & 4,671 & bias & $2,03 E-09$ & 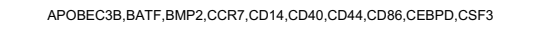 & $172(16)$ \\
\hline Dusp1 & & phosphatase & & $-0,961$ & & $2,04 E-09$ & 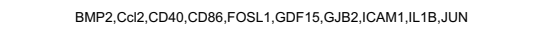 & $210(24)$ \\
\hline F2 & & peptidase & Activated & 4,789 & bias & $2,16 \mathrm{E}-09$ & B4GALT1,BIRC3,CASP3, CC12,CCNA2,CD44,CD68, CDC42EP1,DOCK10,FOSL1 & $168(15)$ \\
\hline 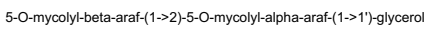 & & hemical - endogenous non-mammalia & Activated & 3,695 & bias & $2,20 \mathrm{E}-09$ & BCL2A1,CCR7,CD40,CD44,CD83,ICAM1, IL18,MGLL,NFKBIZ,RND3 & $174(13)$ \\
\hline แ17A & & cylokine & Activated & 3,673 & bias & $2,21 E-09$ & 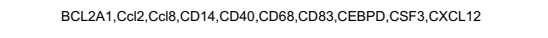 & $144(15)$ \\
\hline tacrolimus & & chemical drug & & 0,392 & bias & $2,39 E-09$ & 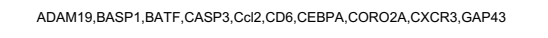 & $223(20)$ \\
\hline thioacetamide & & chemical toxicant & Activated & 3,961 & bias & $2,39 E-09$ & BIRC3,CD144,C224a,CD44,CLDN4,CP,HK1,LL18,JUN,LAMC2 & \\
\hline
\end{tabular}




\begin{tabular}{|c|c|c|c|c|c|c|c|c|}
\hline NFE2L2 & & transcription regulator & Activated & 3,785 & bias & $2,73 E-09$ & AKR1B10,ALAS1,ANG,ARHGEF3,B4GALNT1,CC12,CD86, CEBPA,ESD,FTH1 & $165(17)$ \\
\hline RELA & & transcription regulator & Activated & 4,274 & bias & $2,73 E-09$ & 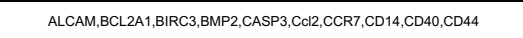 & $187(14)$ \\
\hline SB2203580 & & chemical-kinase innibitior & Inhibited & $-4,228$ & bias & $3,01 E-09$ & 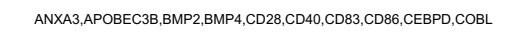 & $187(16)$ \\
\hline TRAF3IP2 & & other & Activated & 3,112 & bias & $3,06 E-09$ & 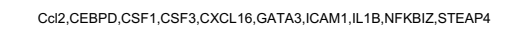 & $172(13)$ \\
\hline phenylbutazone & & chemical drug & Activated & 3,429 & bias & $3,09 E-09$ & CD44,CLDN4,CP,LAMC2,MCM3,MRC1,RCN1,,SLC34A2,TMEM173,TNFRSF $12 A$ & \\
\hline L1 & & group & Activated & 4,968 & bias & 4,08E-09 & 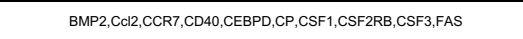 & $176(15)$ \\
\hline Рam3-Cys-Ser-Lys4 & & chemical reagent & Activated & 3,627 & bias & 4,35E-09 & BATF,BCLLA1,CC12,CCR7,CD14,CD40,CD83,CD86,CSF3,HLA-A & $142(14)$ \\
\hline cigarette smoke & & chemical toxicant & Activated & 3,477 & bias & 4,41E-09 & AKR1110,CASP3,CCL1,ESD,FABPF,FOSL1,FTL,GATA3,GSTA3,GSTP1 & $221(17)$ \\
\hline osm & & cytokine & Activated & 2,583 & bias & $5,37 E-09$ & AKR1B10,AMPD3,ANXA3,C1R,CCL2,CEBPA,CEBPD, CLDN4,CSF 3, CXCL12 & $201(20)$ \\
\hline hydrocortisone & & chemical-endogenous mammalian & & 0,731 & & $5,99 \mathrm{E}-09$ & 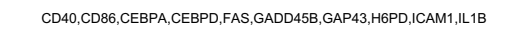 & $177(17)$ \\
\hline СЕВPA & 1,590 & transcription regulator & Activated & 2,552 & bias & 6,41E-09 & ACOD1,AKR1110,BCL2A1,CCNA2,CD14,CEBPA,CEBPD,CSF1,CSF3,,СРP3A7 & $203(20)$ \\
\hline cycloheximide & & chemical reagent & & 0,279 & & $6,64 \mathrm{E}-09$ & 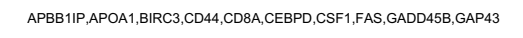 & $193(21)$ \\
\hline CCN5 & & growth factor & & $-1,035$ & & $7,56 E-09$ & CD44,CEBPA,GATA3,JUN,JUP,KLF4,KRT8,LAMB3,LAMC2,SDC4 & $132(7)$ \\
\hline Fos & & transcription regulator & Activated & 2,620 & bias & $8,12 E-09$ & 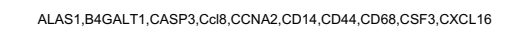 & $211(20)$ \\
\hline indomethacin & & chemical drug & Activated & 2,619 & bias & $8,98 E-09$ & BIRC3,CC12,CD14,CD44,CLDN4,CP,CSF2RB, GDF15,101,1L18 & $228(18)$ \\
\hline hydrogen peroxide & & chemical-endogenous mammalian & Activated & 4,082 & bias & $9,57 E-09$ & 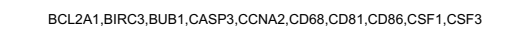 & $143(16)$ \\
\hline PD98059 & & chemical-kinase inhibitor & Inhibited & $-3,828$ & bias & $1,06 E-08$ & 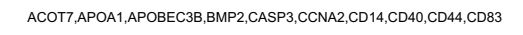 & 161 (19) \\
\hline phenacetin & & chemical drug & Activated & 3,148 & bias & $1,08 E-08$ & BIRC3, СD44,CLDN4,CP,LAMC2,MRC1,SLC34A2,TNFRSF 12A,TSPAN8,UPP1 & $149(7)$ \\
\hline etoposide & & chemical drug & Activated & 3,095 & bias & 1,13E-08 & 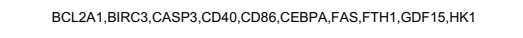 & $133(14)$ \\
\hline calcitiriol & & chemical drug & Activated & 2,383 & & $1,27 E-08$ & ADAM19,APOA 1, ARHGEF 3, BATF, CAVIN1,CCNA2, CD 14,CD40,CEBPA,CEBPD & $208(21)$ \\
\hline $\operatorname{Tnf}$ f(family) & & group & Activated & 2,524 & bias & $1,39 E-08$ & 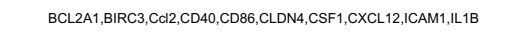 & $135(15)$ \\
\hline EGR1 & & transcription regulator & Activated & 3,371 & bias & $1,48 \mathrm{E}-08$ & 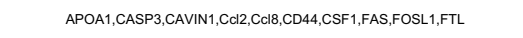 & $226(22)$ \\
\hline RNA polymerase II & & complex & & & & $1,60 E-08$ & ALAS1, BLNKK,CD14,CD40,CD833,CEBPA,CSF1,FOSL1, GADD458, GDF 15 & $180(12)$ \\
\hline PRKCD & & kinase & Activated & 2,910 & bias & $1,75 E-08$ & 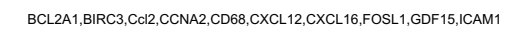 & 159 (17) \\
\hline Tir & & group & Activated & 3,217 & bias & $2,04 E-08$ & 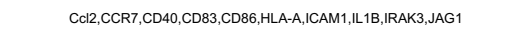 & 141 (18) \\
\hline YAP1 & 1,318 & transcription regulator & & 1,832 & bias & $2,29 E-08$ & 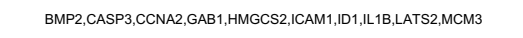 & $206(21)$ \\
\hline IKZF3 & & transcription regulator & Innibitied & $-3,464$ & bias & $2,30 \mathrm{E}-08$ & ANTXR1,BLNK,CAV2,CD68,DEPP1,DOCK4,KLF4,LLFPLL2,MGLL,NFKBBZZ & \\
\hline FAS & 1,934 & transmembrane receptor & & $-1,237$ & & $2,38 E-08$ & BCL11A,BCL2A1,BIRC3,BT & $147(17)$ \\
\hline socs1 & & other & Inhibited & $-3,118$ & bias & $2,95 E-08$ & ACOD1,BCL2A1,CC12,CCR7,CD40,CD44,,CD86,CSF3,CXCR3,FAS & $145(16)$ \\
\hline IRF8 & & transcription regulator & Activated & 3,385 & bias & $3,63 E-08$ & ARIDSB, СASP3,СCR7,CD14,СD40,СD83,СD86,СEBPA,CXCL16,FAS & $199(16)$ \\
\hline TNFRSF1A & & transmembrane receptor & Activated & 3,525 & & $4,06 E-08$ & 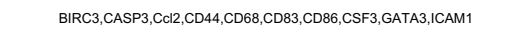 & $143(16)$ \\
\hline TREM2 & & transmembrane receptor & & 0,661 & & $4,10 \mathrm{E}-08$ & COL1,CCR7,CD40,CD86, IL1B,NFKBIA,TNFAIP3 & 150 (14) \\
\hline forskolin & & chemical toxicant & & 1,643 & bias & 4,21E-08 & 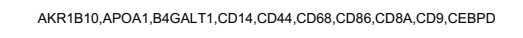 & $239(20)$ \\
\hline Hsp70 & & group & & 0,400 & & $4,29 E-08$ & 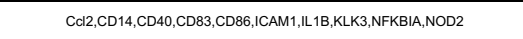 & $137(17)$ \\
\hline L18 & & cytokine & Activated & 4,090 & bias & $4,55 E-08$ & ADAM19,CС12,CCR7,CD40,CD44,CD83,CD86,,CSF1,СXCL12,CXCL16 & 149 (16) \\
\hline Foxo1 & & transcription regulator & Activated & 2,522 & bias & 4,74E-08 & 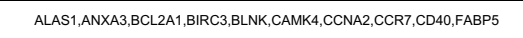 & $193(17)$ \\
\hline МАРзК14 & & kinase & Activated & 3,261 & bias & $5,08 E-08$ & BIRC3,BST1,CC12,CD44,FAS, CCAM1,LL18, ILZR,NFFBBA,RELB & 145 (11) \\
\hline
\end{tabular}




\begin{tabular}{|c|c|c|c|c|c|c|c|}
\hline captopril & chemical drug & Activated & 2,354 & & $5,08 E-08$ & 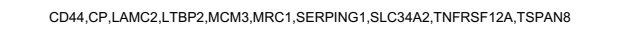 & \\
\hline filgrastim & biologic drug & & $-1,444$ & & $5,49 E-08$ & 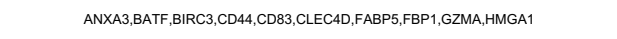 & \\
\hline IFNB1 & cytokine & Activated & 2,766 & bias & $5,54 E-08$ & 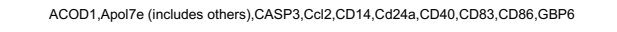 & $168(18)$ \\
\hline МАРКя & kinase & & 1,545 & bias & $5,85 E-08$ & 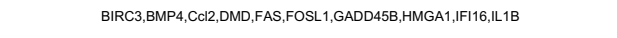 & $216(18)$ \\
\hline 15-deoxy-detta-12,14-PGJ 2 & chemical-endogenous mammalian & & 0,233 & bias & $5,86 E-08$ & 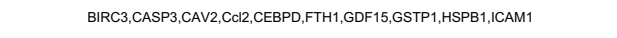 & $227(22)$ \\
\hline resveratrol & chemical drug & & 0,598 & & $5,90 E-08$ & BCL2A1,BIRC3,BMP2,CASP3,CC12,CD 14,CD668,FAS,FOSL1,GDF 15 & 179 (17) \\
\hline clobetasol propionate & chemical drug & & $-1,664$ & bias & $6,38 E-08$ & CD40,CD86,FABP5,FAS,ICAM1, LY75 & $122(10)$ \\
\hline IL12 (complex) & complex & & 1,642 & bias & $7,56 E-08$ & 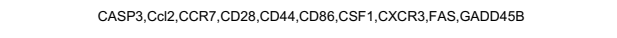 & $153(16)$ \\
\hline methylselenic acid & chemical reagent & & & & 7,65E-08 & 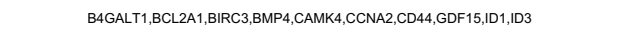 & $241(22)$ \\
\hline NFKB1 & transcription regulator & Activated & 2,882 & bias & $7,73 E-08$ & BCL2A1,BIRC3,BMP2, CCI2,CD40,CD866,CEBPA,CSF1,FAS, GATA3 & $203(14)$ \\
\hline genistein & chemical drug & & 1,628 & bias & $9,44 E-08$ & ALAS1,APOA1,BMP2,BT 33, СASP3, СD44,СD68, СD86, СEBPA,CXCL12 & 190 (21) \\
\hline LYGE & other & & $-1,937$ & & $9,63 E-08$ & CD14,CD83,LL18,IRAK2,NFKBBA,RELB,SERPINE1 & \\
\hline IGF1 & growth factor & Activated & 2,917 & bias & $9,84 \mathrm{E}-08$ & 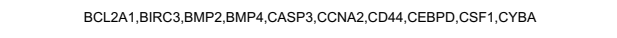 & $215(21)$ \\
\hline SMAD3 & transcripition regulator & & 1,652 & bias & $1,00 E-07$ & 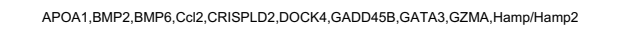 & $199(20)$ \\
\hline VEGFA & growth factor & Activated & 2,219 & bias & 1,02E-07 & ALAS1,ALDH2,BATF,BCLLA1,BMP2,BMP4,CASP3, СD40,CD68,СС83 & $166(17)$ \\
\hline hyaluronic acid & chemical - endogenous mammalian & Activated & 2,762 & bias & $1,05 E-07$ & 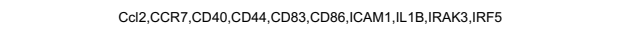 & $147(16)$ \\
\hline N-nitro-L-arginine methyl ester & chemical drug & & 1,439 & & $1,05 E-07$ & 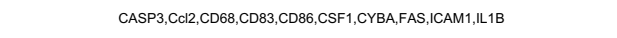 & $191(17)$ \\
\hline PTGER4 & G-protein coupled receptor & & $-1,207$ & & $1,06 E-07$ & $\mathrm{CCl2}, \mathrm{CCR} 7, \mathrm{CD} 40, \mathrm{CD} 44, \mathrm{CP}, \mathrm{GAB} 1, \mathrm{GBP} 6, \mathrm{H} 2-\mathrm{M2}, \mathrm{CCAM1}, 1 \mathrm{~F} 116$ & $193(19)$ \\
\hline dextran sulfate & chemical drug & & 1,012 & bias & $1,07 E-07$ & 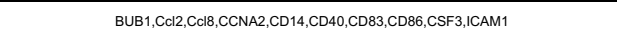 & 154 (17) \\
\hline trogilizzone & chemical drug & & 0,784 & & $1,14 E-07$ & 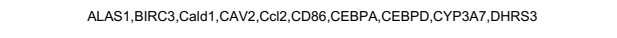 & $207(22)$ \\
\hline mir-223 & microRNA & & 0,697 & & $1,16 \mathrm{E}-07$ & ALCAM,AMPD3,BST1,Clec2d (includes others),CSF1,HPGDS, ICAM1,IF16, LL18,MCTP2 & \\
\hline iron dextran & chemical drug & Activated & 2,391 & bias & $1,16 \mathrm{E}-07$ & 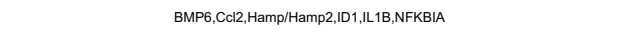 & \\
\hline ADCYAP1 & other & & 0,192 & & $1,19 E-07$ & 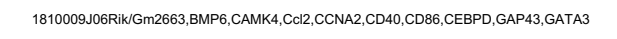 & $167(18)$ \\
\hline bee venom & hemical - endogenous non-mammalia & & 0,507 & bias & $1,19 E-07$ & 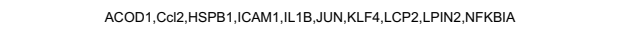 & $117(12)$ \\
\hline LDL & complex & Activated & 3,548 & bias & $1,22 E-07$ & 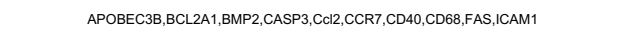 & $179(17)$ \\
\hline TLR3 & transmembrane receptor & Activated & 3,584 & bias & $1,22 E-07$ & 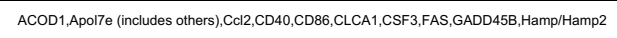 & 141 (16) \\
\hline SHC1 & other & & 1,062 & & $1,40 E-07$ & BMF,CCR7,FTH1,FTL,GSTP1,ICAM1,NQO1,SDC4,SERPINE1,SOD2 & $167(17)$ \\
\hline EGF & growth factor & Activated & 3,789 & bias & $1,44 E-07$ & 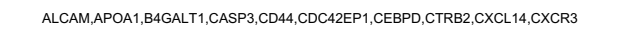 & $192(21)$ \\
\hline SMAD4 & transcription regulator & Activated & 2,366 & bias & $1,56 \mathrm{E}-07$ & 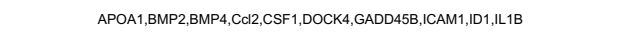 & 259 (24) \\
\hline fasudil & chemical drug & & $-0,800$ & bias & $1,59 E-07$ & BMP2, CASP3, CCI2,CD14,CD40,ICAM1,LL18,JUN,MRC1 & $168(17)$ \\
\hline ZVAD-FMK & chemical - protease inhibitor & & 0,174 & & $1,59 \mathrm{E}-07$ & 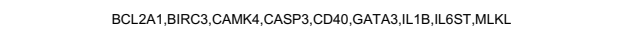 & 154 (17) \\
\hline AGT & growth factor & Activated & 3,140 & bias & $1,62 E-07$ & 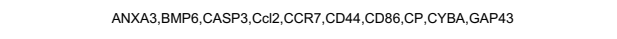 & 195 (18) \\
\hline SMAD5 & transcription regulator & Activated & 2,010 & bias & $1,66 E-07$ & BMP2,CSF1,Hamp/Hamp2,ICAM1, ID1,1D3,RUNX2,SERPINE1 & \\
\hline carboplatin & chemical drug & Activated & 2,530 & bias & $1,68 E-07$ & CASP3, CD44,CLDN4,CP,LAMC2,MCM3,MRC1,SLC34A2,TNFRS 12A,UPP1 & \\
\hline IL21 & cytokine & Activated & 3,374 & bias & $1,78 E-07$ & BATF,СASP3,СCR7,CD2, СD28,CD886,СLC5,СXCR3,,GATA3,,GBP6 & 191 (18) \\
\hline $\mathrm{CD} 3$ & complex & Activated & 3,844 & bias & $1,93 \mathrm{E}-07$ & ALAS1,ANG,BATF,BCLLA1,BRC3,C1R,CASP3, CD2,CD28,CD44 & $168(18)$ \\
\hline IL15 & cytokine & Activated & 2,546 & bias & $1,93 E-07$ & 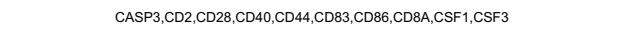 & $166(17)$ \\
\hline
\end{tabular}




\begin{tabular}{|c|c|c|c|c|c|c|c|c|}
\hline pirinixicic acid & & chemical toxicant & Activated & 3,249 & bias & $2,21 E-07$ & 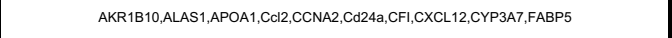 & $218(17)$ \\
\hline Mrc & & transcription regulator & & 1,102 & bias & $2,30 \mathrm{E}-07$ & 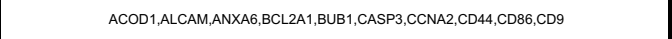 & $229(20)$ \\
\hline caffeic acid phenethyl ester & & chemical drug & & $-1,279$ & bias & $2,72 E-07$ & 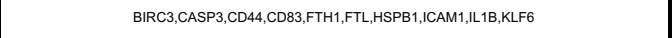 & $147(16)$ \\
\hline RUNX1 & & transcription regulator & & 1,243 & bias & $2,88 \mathrm{E}-07$ & ALCAM, CEBPA,CSF 1,CSF2RB,FAS, GATA3, L1 18,LLRR,ITGA9,LLP2 & $246(25)$ \\
\hline TGFB111 & & transcription regulator & & 0,918 & bias & $2,89 E-07$ & ID1,KLF4,KLK3,KRT19,KRT20,LTBP2,SERPINE1 & $93(7)$ \\
\hline EZH2 & & transcription regulator & & 0,135 & & $2,94 E-07$ & 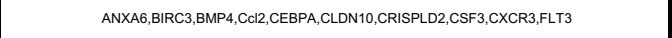 & 231(22) \\
\hline Р38 MAPK & & group & Activated & 3,801 & bias & 3,25E-07 & BCL2A1,BMP2,CCR7,,CD40,CD 44,СD83,CD86,CEBPA,CEBPD,,CSF3 & $158(16)$ \\
\hline TCF4 & & transcription regulator & Activated & 2,550 & bias & 3,48E-07 & ALCAM,ANXAG,CCNA2,CD2,CD9, CEBPA,Clec2d (includes others),CSF3,ENPP, 1,D3 & $209(16)$ \\
\hline Hbb--22 & & other & Activated & 2,236 & bias & $3,64 E-07$ & 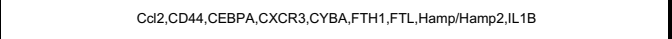 & $138(9)$ \\
\hline D-glucose & & chemical - endogenous mammalian & Activated & 3,388 & bias & $3,72 E-07$ & ANXA3,BMP2,BMP4,CASP3,,CII2,CEBPA,CYBA,DMD,DTNA,FABP5 & $234(21)$ \\
\hline TFAP2C & & transcription regulator & & $-0,990$ & & $3,99 E-07$ & ALCAM,ANTXR2,CD44,GSTA3, ,K1, JAG1,KRTR,MAL,SEMA3B,SOD2 & \\
\hline cyclophosphamide & & chemical drug & Activated & 2,373 & bias & 3,99E- -07 & 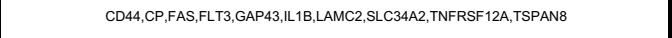 & 191 (17) \\
\hline Mi2 & & other & Inhibitied & $-2,219$ & bias & $4,00 E-07$ & CEBPA,GSTP1,Hamp/Hamp2,L118,JUN,MEST,SERPINE1 & $207(19)$ \\
\hline dihydrotestosterone & & chemical-endogenous manmmalian & Activated & 2,176 & bias & 4,43E-07 & ALAS1,BMF,CASP3,CCNA2,CDCA7,CEBPA,FTH1,FTL,GAP43,GSTP1 & $233(21)$ \\
\hline 1L7 & & cytokine & & 1,347 & bias & $4,72 E-07$ & 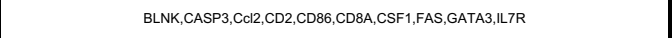 & $181(17)$ \\
\hline Salmonella enterica serotype abortus equil lipopolysaccharide & & chemical toxicant & Activated & 3,978 & bias & $4,99 E-07$ & 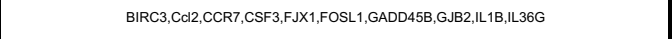 & $163(11)$ \\
\hline PRKAA1 & & kinase & & $-1,291$ & bias & $5,15 E-07$ & 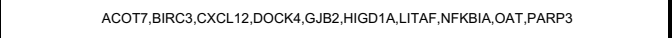 & $170(15)$ \\
\hline paclitiaxel & & chemical drug & Activated & 2,264 & bias & $5,19 E-07$ & 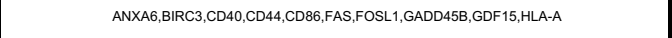 & $204(20)$ \\
\hline PRKAA2 & & kinase & & $-1,038$ & bias & $5,22 E-07$ & ACOT7,ALAL1, СAMK4,CEBPA,CXCL12,DOCK4,GATA3,HIIGD1A,NFKBIA,OAT & $62(5)$ \\
\hline SB-431542 & & chemical reagent & & $-0,851$ & bias & $5,22 E-07$ & 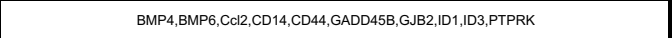 & $211(20)$ \\
\hline WWTR1 & & transcription regulator & & 0,690 & bias & $5,34 E-07$ & BMP2,CCNA2,CD44,ICAM1, L18,LATS2,RUNX2,SERPINE1,YAP1 & $98(8)$ \\
\hline FLT3LG & & cytokine & & 1,640 & bias & $5,44 E-07$ & 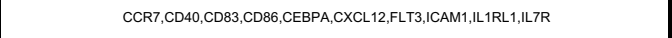 & $200(19)$ \\
\hline IFNAR1 & & transmembrane receptor & Activated & 2,601 & bias & $5,611-07$ & 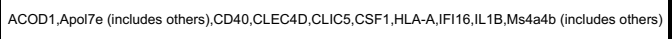 & 180 (18) \\
\hline ACVRL1 & & kinase & & 0,771 & bias & $5,82 E-07$ & CXCL12,GDF 15,ICAM1,ID1,LLRL1,JUP,SERPINE1,ZYX & $143(11)$ \\
\hline MAPKB & & kinase & Activated & 2,000 & bias & 6,33E- -07 & 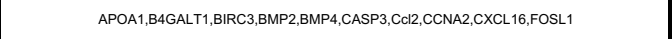 & $218(18)$ \\
\hline CD86 & 2,334 & transmembrane receptor & & 0,727 & bias & 6,43E- -07 & CD28,CD40,CD86,FAS, GZMA,HOMER2,ICAM1,NFKBBA,TNFAIP3 & $154(19)$ \\
\hline LRP1 & 1,226 & transmembrane receptor & & $-1,320$ & & 6,43E- -07 & C1R,CO12,CD14,CD68, L118,LRP1,SERPING1,SLC2A3,SMO & $108(12)$ \\
\hline IRAK4 & & kinase & & 1,938 & bias & $6,84 E-07$ & BIRC3,CC12,CD86,СXCL16,IL18,IRF5,JUN,NFKBIA,NFKBIZ,TNFA|P3 & $175(14)$ \\
\hline RARB & & ligand-dependent tuclear r receptor & & 1,093 & bias & $7,17 \mathrm{E}-07$ & 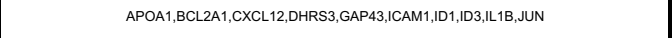 & $220(20)$ \\
\hline HGF & & growth factor & Activated & 2,015 & bias & $7,25 E-07$ & BCL2A1,BIRC3,BMP2,BUB1,,CL12,CD28,CD40, CD44,,CSF1,FOSL1 & $199(18)$ \\
\hline ROсK2 & & kinase & & 0,933 & bias & $7,30 \mathrm{E}-07$ & CCI2,CD44,CEBPA,FABPS,FAS, ICAM1,PRKG1,SERPINE1 & $178(18)$ \\
\hline TLR9 & & transmembrane receptor & Activated & 3,300 & bias & $8,46 E-07$ & 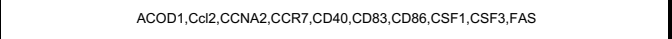 & 155 (17) \\
\hline $\mathrm{Ca}_{2}+$ & & chemical - endogenous mammalian & Activated & 2,534 & bias & $8,58 E-07$ & 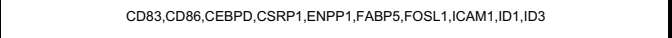 & $189(18)$ \\
\hline oxyletracycline & & chemical drug & Activated & 2,000 & bias & $8,96 E-07$ & ВMP2,ВMPA,BMP, ID1 & \\
\hline ECSIT & & transcription regulator & Activated & 2,567 & bias & $9,73 \mathrm{E}-07$ & CD44,CD83, IL1B,NFKBIA,RELB,SOD2,TNFAPP3 & $151(16)$ \\
\hline TGFBR2 & & kinase & & 0,605 & & $1,011-06$ & 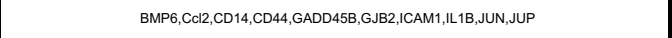 & 209 (21) \\
\hline ERK & & group & Activated & 3,110 & bias & 1,02E-06 & 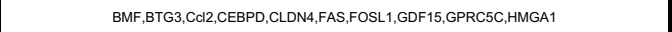 & $214(21)$ \\
\hline
\end{tabular}




\begin{tabular}{|c|c|c|c|c|c|c|c|c|}
\hline NFATC2 & & transcription regulator & & $-0,336$ & bias & $1,08 E-06$ & 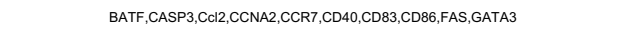 & $172(18)$ \\
\hline TNFRSF9 & & transmembrane receptor & & $-0,342$ & & $1,12 E-06$ & BCL2A1,CD14,CD28,CD40,CD83,СD86,FAS, L118 & $155(16)$ \\
\hline CTNNB1 & & transcription regulator & Activated & 3,308 & bias & $1,22 E-06$ & ALCAM,BMF,BMP2,BMPР,,CCNA2, СD44,CEBPA,CSFF3,CTSZ,,CXCL12 & 199 (19) \\
\hline TRADD & & other & & 1,890 & bias & $1,27 E-06$ & 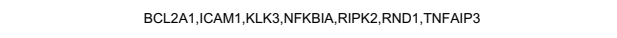 & $140(15)$ \\
\hline DACH1 & & transcription regulator & & $-1,276$ & bias & $1,38 E-06$ & BMPA, ID1,JUN,KLFA4,KKK3,SERPINE1,STC2,TNFAPP3 & $206(16)$ \\
\hline Ifn & & group & Activated & 3,111 & bias & $1,41 E-06$ & CD40,CD44,CD83, CD86, CXCR33,FAS, HLA-A,ICAM1, F116, OASL & 158 (19) \\
\hline palmitic acid & & chemical-endogenous mammalian & & 1,933 & bias & 1,43E-06 & 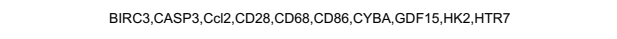 & $199(18)$ \\
\hline $\mathrm{NKX} 2-1$ & & transcription regulator & & 0,958 & bias & 1,45E-06 & ВСС2A1,BMPA,BTG3,CCKAR,CD14,CEBPA,HLA-A,LCKK,Nes, RNASE4 & \\
\hline CTLA4 & & transmembrane receptor & & $-1,832$ & bias & $1,51 E-06$ & CCNA2,CD40,CD83, CD886,GATA3,ICAM1,_LAG3,PAD12,PRKD3,SOD2 & 151 (17) \\
\hline TRAF6 & & enzyme & Activated & 2,382 & bias & $1,51 E-06$ & BIRC3,CCI2,CSF3,CXCL16,ICAM1,LL18,IRF5, RELB,SOD2,TNFAIP3 & $155(14)$ \\
\hline lipoteichoic acid & & hremical - endogenous non-mammalia & Activated & 2,203 & bias & $1,51 E-06$ & ANTXR1,ANTXR2,CO12,CD14,CD83, CD86, CSF3,ICAM1,L118,NOD2 & $133(17)$ \\
\hline$F 2 R$ & & G-protein coupled receptor & & 0,854 & bias & $1,51 E-06$ & 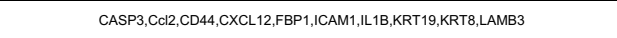 & $237(24)$ \\
\hline iron & & chemical-endogenous mammalian & & 1,537 & & $1,52 E-06$ & BMP, СEBPA,FTH1,FLL,GADD45B, Hamp/Hamp2,101,SLC11A2,SOD2 & 129 (9) \\
\hline tetrachlorodibenzzodioxin & & chemical toxicant & & 0,648 & & $1,53 \mathrm{E}-06$ & 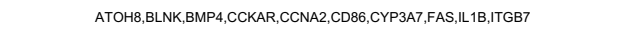 & $200(20)$ \\
\hline TLR2/3/4/9 & & group & Activated & 2,207 & bias & $1,56 E-06$ & CCR7,CD40, СD83, CD866,ICAM1 & $102(7)$ \\
\hline N-acety-l-cysteine & & chemical drug & Inhibitied & $-3,741$ & bias & $1,62 E-06$ & 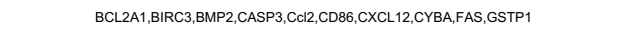 & 189 (18) \\
\hline Mt1 & & other & & $-1,408$ & bias & $1,65 E-06$ & CEBPA,GSTP1,LL18,JUN,MEST,NFKBIA,SERPINE1 & $181(15)$ \\
\hline NR5A2 & & \begin{tabular}{|l|l|} 
Iigand-dependent nuclear receptor \\
\end{tabular} & & 1,177 & bias & $1,68 E-06$ & 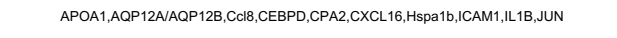 & $172(14)$ \\
\hline TP63 & & transcription regulator & Activated & 3,116 & bias & $1,71 E-06$ & 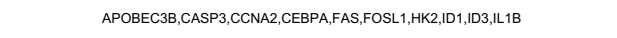 & $216(22)$ \\
\hline E. coli serotype 012788 lipopolysaccharide & & hremical - endogenous non-mammalia & Activated & 3,500 & bias & $1,80 E-06$ & 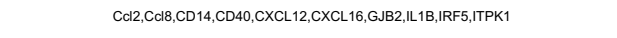 & $174(20)$ \\
\hline TWIST1 & & transcription regulator & & 0,421 & bias & $1,89 E-06$ & 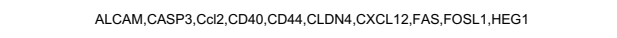 & 180 (17) \\
\hline curcumin & & chemical drug & Inhibited & $-2,347$ & bias & $1,90 E-06$ & A4GALT, BIRC3,CASP3,CC12,CD44,CD81,CD86, СEBPA,CXCR3,FAS & $192(18)$ \\
\hline CD2 & 3,268 & transmembrane receptor & Activated & 2,433 & bias & $2,04 E-06$ & CCNA2,CCR7,CD40,CD44,CD86, CD8A,FAS,ICAM1 & 155 (18) \\
\hline ethanol & & chemical- endogenous mammalian & & 1,935 & bias & $2,11 E-06$ & 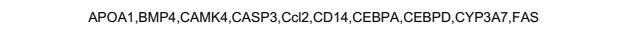 & $218(19)$ \\
\hline SATB1 & & transcription regulator & & 0,874 & & $2,20 E-06$ & 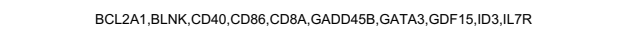 & $103(7)$ \\
\hline FGF7 & & growth factor & Activated & 2,447 & bias & $2,25 E-06$ & 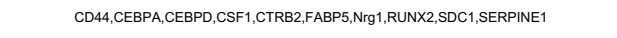 & 189 (19) \\
\hline BIRC3 & 0,908 & enzyme & & $-1,633$ & bias & $2,32 E-06$ & BIRC3,CSF1,CSF3,ICAM1,L118,NFKBIA & 148 (14) \\
\hline Nos2 & & enzyme & & 1,546 & & $2,37 E-06$ & 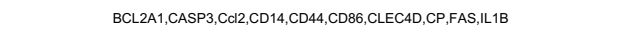 & $134(13)$ \\
\hline pyrrolidine dithiocarbamate & & chemical reagent & Inhibited & $-2,759$ & bias & $2,51 E-06$ & 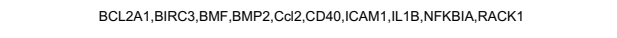 & $120(14)$ \\
\hline ATG7 & & enzyme & & 1,294 & & $2,57 E-06$ & 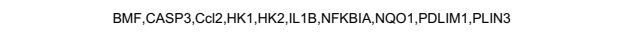 & $185(15)$ \\
\hline simvastatin & & chemical drug & & $-0,174$ & & $2,57 E-06$ & 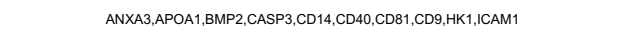 & $136(14)$ \\
\hline RBPJ & & transcription regulator & Inhibited & $-2,735$ & bias & $2,60 E-06$ & 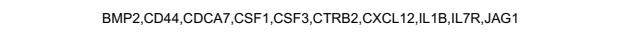 & 198 (24) \\
\hline REL & & transcription regulator & Activated & 3,080 & bias & $2,64 E-06$ & BCL2A1, CD40, CD86, CLDN10,FAS,FOSLL, GADD458, HLA-A,ICAM1,IRF5 & $172(17)$ \\
\hline WNT3A & & cytokine & Activated & 2,120 & bias & $2,75 E-06$ & ВMP2,BMP4,CAMK4,CD44,CD68,,СEBPA,CXCL 12, GAP43,ID1,JAG1 & $188(17)$ \\
\hline Akt & & group & & 0,852 & bias & $2,76 E-06$ & BCL2A1,BMF,BMP2,CCL1, CCR7,CD44,CP,CXCL12,FAS,FOSL1 & $199(20)$ \\
\hline TRAF3 & & enzyme & & $-0,684$ & bias & $2,87 E-06$ & BIRC3,CXCL12,FAS, ICAM1, I1 18,NFKBIA,PTPN14, RELB,RHBDF1,Serpina3g (includes others) & $114(13)$ \\
\hline NOD2 & 2,190 & other & Activated & 2,807 & bias & $2,91 E-06$ & 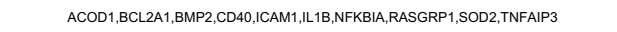 & 118 (13) \\
\hline
\end{tabular}




\begin{tabular}{|c|c|c|c|c|c|c|c|c|}
\hline estrogen receptor & & group & & $-0,545$ & & $2,97 E-06$ & CAV2, CD44,CD68, CLDN4,CXCL12,F11R,GATA3,ICAM1,JAG1,JUN & $244(24)$ \\
\hline RAF1 & & kinase & Activated & 2,761 & bias & 3,11E-06 & ACOT7,CD44,CDC42EP1,DHRS3,FAS,HSPB 1,ID3, IL18,JUN,MGLL & $227(22)$ \\
\hline erlotinib & & chemical drug & Activated & 2,391 & bias & $3,19 E-06$ & CD14,CD40,CSF3,FAS, ICAM1,LL18,NFKBAA,RELB,SERPINE1,SLCCA3 & $192(17)$ \\
\hline PRKCB & & kinase & Activated & 2,646 & bias & $3,27 E-06$ & CD40, СD83, СD86, ICAM1, JUN, RELB, SERPINE1,SOD2,TCIRG1 & 139 (15) \\
\hline SMADT & & transcription regulator & & $-1,932$ & bias & 3,30E-06 & 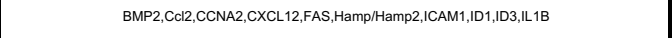 & $178(20)$ \\
\hline FOSL1 & 1,319 & transcription regulator & & 1,463 & & $3,30 \mathrm{E}-06$ & CC12,COC18,CD44,CSF3,FFSL1,1118, JUN,RUNX2,SERPINE1,SERPINE2 & $185(20)$ \\
\hline bisindolylmaleimide I & & chemical - kinase inhibitor & Inhibited & $-3,420$ & bias & $3,36 \mathrm{E}-06$ & 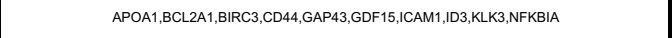 & 171 (17) \\
\hline lipid A & & chemical toxicant & Activated & 2,789 & bias & 3,52E- -06 & 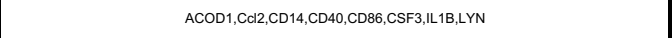 & $134(18)$ \\
\hline $\operatorname{Irgm} 1$ & & other & Inhibitied & $-2,781$ & bias & 3,52E-06 & BUB1,CCI2,CCNA2,FAS, ID3, IF1616,NEK2, OASL & $106(7)$ \\
\hline Y 27632 & & chemical drug & & $-1,601$ & bias & 3,73E-06 & 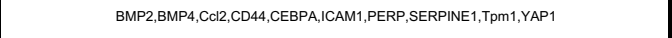 & $148(18)$ \\
\hline mir-125b-5p (and other miRNAs w/seed CCCUGAG) & & mature microRNA & Inhibited & $-3,273$ & bias & $3,95 E-06$ & BMF,CCNA2,CD44,HK2,ID1,1D3,JUN,KRT19,PERP,SMO & $160(7)$ \\
\hline thermozymocidin & & chemical reagent & & $-1,195$ & bias & $4,20 \mathrm{E}-06$ & APOA1,CD86,IL1B,MRC1,TNFAIP3 & $84(5)$ \\
\hline ALOX15 & & enzyme & & 1,141 & bias & 4,21E-06 & CO12,FOSL1,ICAM1,ID3,LL18,JUN,Muc1 & $184(18)$ \\
\hline CXCL12 & 1,946 & cytokine & Activated & 3,915 & bias & 4,33E-06 & BMP2,BMPG, CD14,CD44,CD9, CXCL12,FAS,ICAM1,1D1,FNGR2 2 & $147(15)$ \\
\hline SP600125 & & chemical -kinase inhibitor & & $-1,312$ & bias & $4,40 \mathrm{E}-06$ & 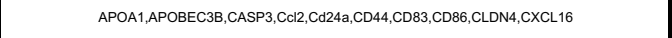 & $159(16)$ \\
\hline SIRT1 & & transcription regulator & Inhibited & $-2,157$ & bias & 4,41E-06 & 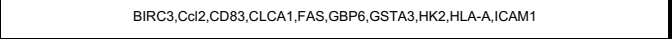 & $200(20)$ \\
\hline RAS & & group & & 0,000 & bias & $4,47 E-06$ & 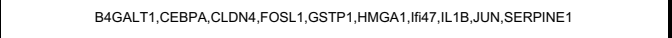 & $212(22)$ \\
\hline ELAVL1 & & other & Activated & 3,543 & bias & 4,92E- -06 & 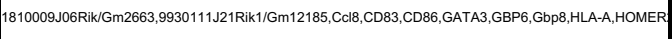 & \\
\hline IRF3 & & transcription regulator & Activated & 2,324 & bias & 4,92E- -06 & 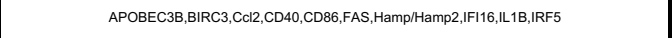 & $183(19)$ \\
\hline TNFAP 3 & 0,947 & enzyme & & $-1,309$ & bias & $4,96 E-06$ & 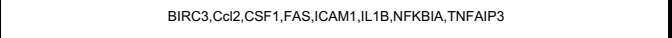 & $148(13)$ \\
\hline C3AR1 & & G-protein coupled receptor & & 1,002 & bias & 4,96E-06 & 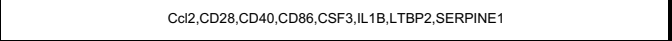 & $160(18)$ \\
\hline TYROBP & & transmembrane receptor & Activated & 2,608 & bias & $5,00 E-06$ & CCR7, CD40, СD83, CD86, ICAM1,LL18,LHFPL2,NOD2,TCIRG1 & $162(19)$ \\
\hline brostatin 1 & & chemical drug & & 1,892 & bias & $5,20 \mathrm{E}-06$ & BIRC3,CD40,CD44,CD86,ICAM1,IFNGR2,IL1B & $153(18)$ \\
\hline 5-azacytidine & & chemical drug & Activated & 3,657 & bias & $5,21 E-06$ & BTG3, CAVIN1,CD44,CXCL 12,FOSL1,GDF 15,GSTP1,HK2,KLF4, KLF6 & $171(11)$ \\
\hline ESR2 & & ligand-dependent nuclear receptor & & 1,603 & & $5,31 E-06$ & 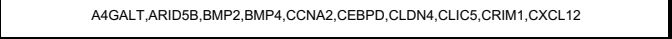 & $227(20)$ \\
\hline PPP3CA & & phosphatase & & & & 5,32E-06 & B3GAT1,CD2,CD9, CSRP 1,FTL,GSTP1,KRT, LITAF, RUNX2,SDC4 & \\
\hline DYSF & & other & & & & $5,32 E-06$ & CCl8,CD68, СD83,СYBA,FTL,HLA-A,HSPB1, IF116,MRC1, TMEM123 & \\
\hline MAР3K7 & & kinase & & 1,949 & bias & $5,32 E-06$ & BIRC3,CCI2,CCR7,CD14,LL18,JUN,OASL,RNASELL,Saa3,SERPINE1 & $177(16)$ \\
\hline AGN194204 & & chemical drug & Activated & 2,868 & bias & $5,37 E-06$ & ADAM19,BCL2A1,CCNA2,Cd24a,CD66,CEBPA,CSRP1,Fmn11,FNBP1,GATA3 & \\
\hline ВМР6 & 1,628 & growth factor & Activated & 2,245 & bias & 5,39E- -06 & 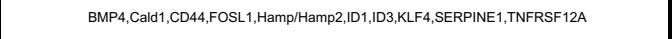 & $199(20)$ \\
\hline bucladesine & & chemical toxicant & & 1,104 & bias & $5,41 E-06$ & AKR1110,CD14,CO224a,CD44,CD86,,CD8A,DMD,ENPP1,FOSL1,GADD45B & $231(21)$ \\
\hline ETV5 & & transcription regulator & & 0,053 & & $5,96 E-06$ & ALCAM,CAVIN1, CLDN4,CLLC5, СXCL12,ICAM1,KRT19,KRT23, SLC 34A2, TINAGL1 & \\
\hline recombinant human endostatin & & chemical drug & & 0,849 & bias & 6,07E- -06 & CASP3,FAS,JUN,KLK3 & \\
\hline murabutide & & chemical reagent & & 1,000 & bias & $6,07 E-06$ & $\mathrm{CD} 40, \mathrm{CD} 83, \mathrm{CD} 86, \mathrm{MRC} 1$ & $156(15)$ \\
\hline let-7 & & microRNA & Inhibited & $-2,921$ & bias & 6,33E-06 & 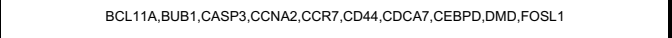 & $118(9)$ \\
\hline Mek & & group & & 0,899 & bias & $6,40 \mathrm{E}-06$ & 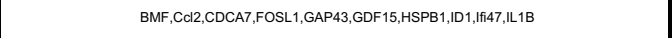 & $229(21)$ \\
\hline epigallocatechin-gallate & & chemical drug & & $-0,165$ & bias & $6,40 \mathrm{E}-06$ & 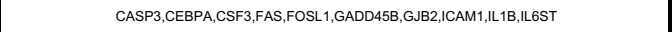 & $203(22)$ \\
\hline
\end{tabular}




\begin{tabular}{|c|c|c|c|c|c|c|c|c|}
\hline MvP & & other & & & & 6,42E-06 & CD83,CD86,ICAM1, L118,JUN & $223(20)$ \\
\hline gamma-tocotrienol & & chemical drug & & 0,251 & bias & $6,42 E-06$ & CSF3,L1B,UUN,NFKBAA,TNFAPB 3 & $128(13)$ \\
\hline nitric oxide & & chemical-endogenous manmmalian & & 1,586 & bias & 6,49E-06 & 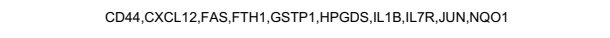 & $230(23)$ \\
\hline napabucasin & & chemical drug & Inhibitied & $-3,000$ & bias & $6,54 E-06$ & ALCAM, CD44,ID1,JAG1, KLF4,NOTCH2,SMO,WWC1,YAP1 & \\
\hline Sn50 peptide & & chemical toxicant & Inhibitied & $-2,942$ & bias & $6,54 \mathrm{E}-06$ & BCL2A1,EIRC3, CCI2,FAS,ICAM1,IFNGR2,IL18,RACK1,SERPINE1 & $140(14)$ \\
\hline soD1 & & enzyme & & $-1,982$ & bias & $6,56 \mathrm{E}-06$ & 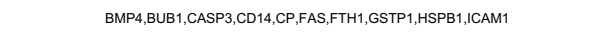 & $191(17)$ \\
\hline BMP2 & 3,424 & growth factor & Activated & 2,593 & bias & $6,74 E-06$ & BMP2,BMP4,BTG3, Cald1, CAMK4,CSF1,CXCL12, GADD458, Hamp/Hamp2, ID1 & $185(19)$ \\
\hline RARA & & ligand-dependent tuclear r receptor & & 0,258 & & $6,76 \mathrm{E}-06$ & APOA 1, CCNA2, CD14,CDA4, CDO, CEBPA, CLMN, COBL,CP, CSF3 & $230(22)$ \\
\hline PRDM1 & & transcription regulator & & $-1,661$ & & $6,78 E-06$ & 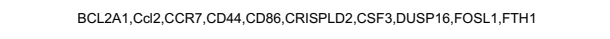 & $232(18)$ \\
\hline cephaloridine & & chemical drug & Activated & 3,283 & bias & $7,26 \mathrm{E}-06$ & AKR1B10,ALAS1,CXCL14,GIycam1,GSTP1,LAMB33,MCM3,NQ01,PIR,RND1 & \\
\hline PP2/AG1879 tyrosine kinase inhibitor & & chemical -kinase inhibitor & Inhibitied & $-2,983$ & bias & $7,48 E-06$ & BIRC3,CXCL16,ICAM1,ID1,LL18,JUP,KLK33,LYN,SERPINE1,SOD2 & 221 (19) \\
\hline MTPN & & transcription regulator & Activated & 3,130 & bias & $7,48 E-06$ & BCL2A1,CASP3,CII2,CCNA2,CEBPA,CP,FAS,HGPD,JUN,SERPINE1 & $102(10)$ \\
\hline oxazolone & & chemical reagent & & 1,289 & bias & $7,52 \mathrm{E}-06$ & CCl2, GIycam1,ICAM1,LL18,SDC1,SDC4 & $94(7)$ \\
\hline CD44 & 0,486 & other & Activated & 2,897 & bias & 7,62E-06 & BIRC3,CC12,CCR7,CD44,CD8A,CXCR3,FAS, I118,IRAK3,JUN & $171(18)$ \\
\hline BMP4 & 5,064 & growth factor & Activated & 2,817 & bias & $7,62 E-06$ & 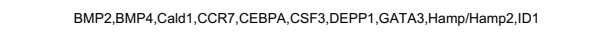 & $180(18)$ \\
\hline TFRC & & transporter & & $-1,726$ & bias & $8,35 E-06$ & CASP3,CCNA2,FTH1,FTL,HK2,JUN,LY775,SLC11A2,STC2,TNFRSF12A & $43(3)$ \\
\hline infliximab & & biologic drug & Inhibited & $-3,000$ & bias & $8,48 E-06$ & 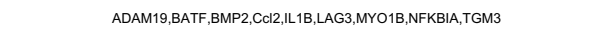 & $124(11)$ \\
\hline vegf & & group & Activated & 4,094 & bias & $8,51 E-06$ & BCL2A1,BMP2,BUB1,CASP3, СC12,CD40, СD44,CXCL12,FAS,FOSL1 & $160(17)$ \\
\hline sP2509 & & chemical reagent & & $-0,447$ & bias & $8,74 E-06$ & BCL11A,CCNA2,,CDCA7,DHRS3,DOCK1,GAP43,,GDPD5,ID1,KLF4,MICALL2 & \\
\hline MR17HG & & other & Inhibited & $-2,887$ & bias & $9,01 E-06$ & ВMP4,HEG1,LLST, LIRR,JAG1,LRP1,NOTCH2,PTK7,PTPRK,SSEMA3E & \\
\hline PRKCA & & kinase & & 0,595 & bias & $9,01 E-06$ & 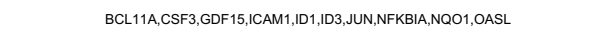 & $203(23)$ \\
\hline celecoxib & & chemical drug & & 0,708 & bias & $9,01 E-06$ & 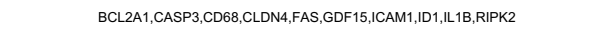 & $197(20)$ \\
\hline valproic acid & & chemical drug & & 0,347 & & $9,29 E-06$ & AMPD3,ANTXR2,APOA1,B4GALT1, CASP3, CD40,CD44,CD83,CDCA7,CEBPA & $207(20)$ \\
\hline Ітк & & kinase & & 0,708 & bias & $9,31 E-06$ & CD44, ,CRIM1,FAS, GATA3,HLA-A,LLYN,PREX1,Serpina39g (includes others),SRGAP3,ZZTTB16 & $138(8)$ \\
\hline PTGS2 & & enzyme & Activated & 3.642 & bias & 9,43E-06 & ANG,BIRC3,CCL12,CCR7,CD44,CD68, CSF3,CXCL 12,CXCL14,ICAM1 & 175 (19) \\
\hline IKZF2 & & transcription regulator & Inhibited & $-2,646$ & bias & 9,43E-06 & BATF,CD2, CD28,,GATA3,GZMA,ICAM1,_LAG3 & \\
\hline ATP-gamma-S & & chemical reagent & & & & $9,62 E-06$ & CCR7,CD40,СD83,СD86,,СD8A,CSF 1,,GAP43, ILRR,SOD2 & $165(17)$ \\
\hline Nfat (family) & & group & Activated & 3,000 & bias & $9,67 E-06$ & B4GALIT1,CD40,DOCK10,FAS,ICAM1,KLFG,LAG3,RELL,SDC4,SERPINE1 & 178 (21) \\
\hline XIAP & & enzyme & & $-0,156$ & bias & 9,72E-06 & ACOD1,CASP3,CSF1,CSF3,SERPINE1,SOD2 & $161(16)$ \\
\hline sirolimus & & chemical drug & Inhibited & $-2,124$ & bias & $9,84 E-06$ & 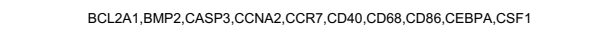 & $164(18)$ \\
\hline nitrofurantioin & & chemical drug & & 1,437 & & $9,92 E-06$ & 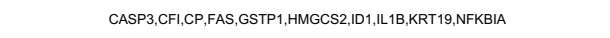 & \\
\hline $\lg G$ & & complex & & $-0,822$ & bias & 1,03E-05 & 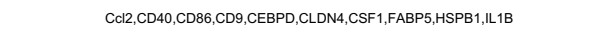 & 139 (18) \\
\hline histamine & & chemical-endogenous mammalian & Activated & 2,283 & bias & $1,04 E-05$ & 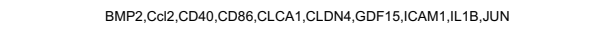 & $219(21)$ \\
\hline CAV1 & & transmembrane receptor & Activated & 2,621 & bias & $1,06 \mathrm{E}-05$ & 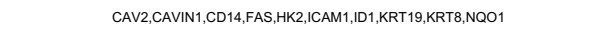 & $199(22)$ \\
\hline VDR & & transcripition regulator & & 1,291 & & 1,06E-05 & 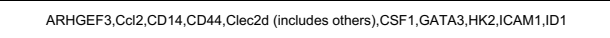 & $188(20)$ \\
\hline BCL3 & & transcription regulator & & 0,105 & & $1,08 E-05$ & CD40,CD86, GATA3,ICAM1, ID1, LL 18, IL36G,JUN & $152(14)$ \\
\hline IRF6 & & transcription regulator & & 0,114 & bias & $1,08 E-05$ & 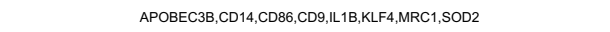 & 141 (16) \\
\hline
\end{tabular}




\begin{tabular}{|c|c|c|c|c|c|c|c|c|}
\hline trovafloxacin & & chemical drug & Activated & 2,333 & bias & $1,09 E-05$ & BIRC3,CEBPA,HLA-A,ICAM1,NFKBAA,SERPINE1,SLC11A2,SOD2,TNFAIP3 & $187(11)$ \\
\hline CYLD & & transcription regulator & & 1,031 & & 1,14E-05 & CD40,CD86, L1 18,LLRR,JUN,RELB, SERPINE1 & $119(14)$ \\
\hline ADORA2B & & G-protein coupled receptor & & $-0,114$ & bias & $1,14 E-05$ & COI2,CD86,IL18,KLF4,MRC1,RUNX2,SERPINE1 & $151(19)$ \\
\hline FGF1 & & growth factor & & $-0,996$ & & $1,16 E-05$ & 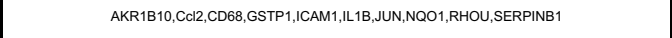 & $167(17)$ \\
\hline arachidonic acid & & chemical-endogenous mammalian & & 0,092 & & $1,16 \mathrm{E}-05$ & 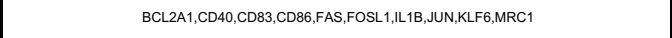 & $175(18)$ \\
\hline HDAC1 & & transcription regulator & & $-1,141$ & & $1,17 E-05$ & AMPD3,APOA 1,BMF,CCNA2,CD44,CEBPA,FAS, GSTP1,1,11,KLK3 & $215(21)$ \\
\hline androgen & & chemical drug & & $-1,671$ & & $1,22 E-05$ & BMP4,BMPG, CDA4,CTSZ,FOSL1, JUN,KLKK3,NFKBIA,PTPAA2,PTPRK & $190(20)$ \\
\hline SP3 & & transcription regulator & Activated & 2,195 & bias & $1,23 E-05$ & BMF,CCL1, CD40,CEBPA,DMD,FAS,FOSL1,GDF 151,JUN, KLF4 & $216(20)$ \\
\hline JAK1 & & kinase & Activated & 2,937 & bias & $1,23 E-05$ & 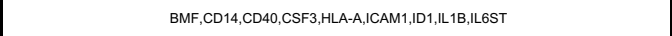 & $179(18)$ \\
\hline IRAK3 & 0,720 & kinase & Activated & 2,429 & & $1,24 \mathrm{E}-05$ & BCLZA1, CCL2, IRF5,NFKBIA,SOD2, TNFAIP3 & 165 (15) \\
\hline bromodeoxyuridine & & chemical drug & Activated & 2,813 & bias & $1,25 E-05$ & 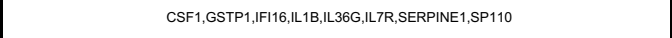 & $173(11)$ \\
\hline thapsigargin & & chemical toxicant & Activated & 2,340 & bias & $1,29 E-05$ & 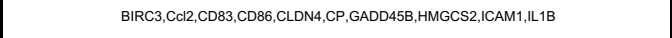 & $171(19)$ \\
\hline LDLR & & transporter & Inhibitied & $-2,078$ & bias & $1,30 \mathrm{E}-05$ & ALCAM, $, \mathrm{BMP} 2, \mathrm{CC1} 2, \mathrm{CCl}, \mathrm{CCNA2}, \mathrm{CD} 40,, \mathrm{CD} 68, \mathrm{CEBPA}, \mathrm{FABPP}, \mathrm{HGPD}$ & $172(16)$ \\
\hline LF & & cylokine & Activated & 2,341 & bias & $1,30 \mathrm{E}-05$ & 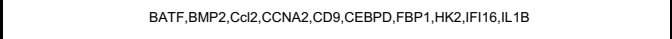 & $217(22)$ \\
\hline PPARA & & ligand-dependent nuclear r receptor & & 0,230 & bias & $1,30 \mathrm{E}-05$ & ALDH2,APOA1,C1R,CCI2,CCNA2,CEBPA,CEBPD, CFI,CXCL12,CYBA & $192(17)$ \\
\hline lenalidomide & & chemical drug & Activated & 3,183 & & $1,32 E-05$ & ANTXR1,BLNK,CAVV2,CD68, CEBPA,DEPP 1,DOCK4,DTNA,GPR68, KLF4 & $35(3)$ \\
\hline Histone h3 & & group & & & & $1,35 E-05$ & 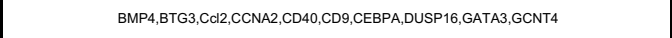 & $210(19)$ \\
\hline S1PR3 & & G-protein coupled receptor & & 0,372 & bias & $1,35 E-05$ & CCl2,CD44,ICAM1, IL1B,JUN & $192(19)$ \\
\hline WNTSA & & cyokine & & 1,303 & & $1,36 \mathrm{E}-05$ & 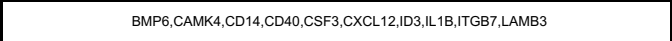 & $226(21)$ \\
\hline CSF1 & 0,920 & cytokine & Activated & 3,518 & bias & $1,38 E-05$ & 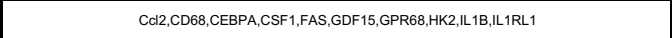 & $209(21)$ \\
\hline pioglitazone & & chemical drug & & $-0,292$ & & $1,39 E-05$ & 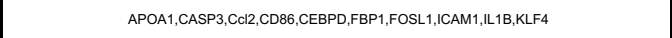 & $160(18)$ \\
\hline GLI2 & & transcription regulator & Activated & 2,000 & bias & $1,39 \mathrm{E}-05$ & 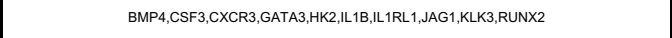 & $164(10)$ \\
\hline MYoc & & other & & & & $1,41 E-05$ & CD44,JDP2,LAMA2,PXON,RAB278, RHBDF1,RUNX2,SLC2A3,STCZ,UPP1 & \\
\hline TGFBR1 & & kinase & & 1,541 & bias & 1,41E-05 & BMP4,CCR7,CD40,CD86,DOCK4,Hamp/Hamp2,JAG1,KLF4,PTPRK,SERPINE1 & $219(21)$ \\
\hline MTOR & & kinase & & 1,389 & bias & $1,57 E-05$ & 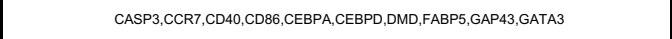 & $242(21)$ \\
\hline МАРЗКв & & kinase & & 1,101 & & $1,65 E-05$ & 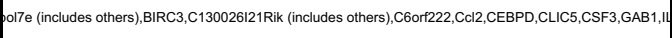 & $187(18)$ \\
\hline KDMAA & & enzyme & & $-0,285$ & & $1,69 \mathrm{E}-05$ & 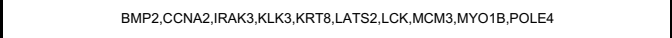 & $120(7)$ \\
\hline sulforatan & & chemical drug & & 1,234 & & $1,72 E-05$ & 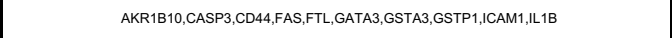 & $185(14)$ \\
\hline benzo(a)pyrene & & chemical toxicant & & 0,578 & bias & $1,72 E-05$ & APOA1,BTG3,CASP3,ESD,GSTP1,HK2,HSPP 16, HSPB1, IL1B,JUN & $225(22)$ \\
\hline tazemetostat & & chemical drug & & $-0,229$ & bias & $1,73 E-05$ & BCL11A,CDCA7,DHRS3,DOCK1,GAP43, GDPD5, ID1,KLF4,MICALL2,MY01B & \\
\hline ZFPM1 & & transcription regulator & & & & $1,75 E-05$ & BLNK,,CEBPA,ENPP1,GATA3, RAAK3,KLFG,PLA2616,SCIN,SH2DAA & $21(2)$ \\
\hline GRRH-A & & chemical reagent & Activated & 2,971 & & $1,75 E-05$ & AKR1110,CD68,FOSL 1,GADD45B,JUN,KLF4,KLFF,NFKBBZ,SERPINE1 & $212(18)$ \\
\hline TREM1 & & transmembrane receptor & Activated & 2,438 & bias & $1,75 E-05$ & CO12,CCR7,CD14,CD83,CD86,CSF1,FOSL1,GADD458,LL18, ,L1RL1 & $186(15)$ \\
\hline deferroxamine & & chemical drug & Activated & 3,539 & bias & $1,75 E-05$ & CCR7,CD14,CP,DUSP16,GADD45B, GDF 15,HK2,ICAM1,IF16,ITGB7 & $204(21)$ \\
\hline CHD1 & & enzyme & Activated & 2,236 & bias & $1,88 E-05$ & BCL2A1,BMP2, ICAM1,LL1,SOD2 & \\
\hline cytokine & & group & Activated & 3,028 & bias & $1,89 \mathrm{E}-05$ & ANG,BMF,CCR7,CD40,CDB6, CSF1,FAS,ICAM1,JUN,RIIFK2 & $148(17)$ \\
\hline GDF2 & & growth factor & Activated & 3,055 & bias & $1,90 \mathrm{E}-05$ & 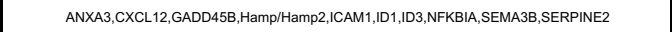 & 142 (11) \\
\hline
\end{tabular}




\begin{tabular}{|c|c|c|c|c|c|c|c|}
\hline AGTR1 & G-protein coupled receptor & & 0,816 & bias & $1,92 E-05$ & CASP 3, CP,CYBA,GSTP1,JUN,SERPINE1,XDH & $197(20)$ \\
\hline geftitinib & chemical drug & & 0,776 & bias & $1,93 E-05$ & BDH2,BMF,,BUB1,CC12,CEBPD,FOSL1,GADD458,HSPB1,1D1,KRT19 & $179(16)$ \\
\hline E. coll ilipopolysaccharide & hemical - endogenous non-mammalia & Activated & 2,414 & bias & $1,96 E-05$ & CC12,CCR7,CD40,CD83,CD86,CEBPA,CEBPD, ICAM1,LL1B & $150(17)$ \\
\hline PAX7 & transcription regulator & Activated & 2,759 & bias & $1,96 E-05$ & CAMK 1D,CD24a, ID3, Marcks, MEST,MYO1D,NQO1, RASA3,SEMA3E & \\
\hline peptidoglycan & |hemical-endogenous non-mammalia & Activated & 3,246 & bias & $1,98 \mathrm{E}-05$ & BCL2A1,CASP3,CCL12,CD40,,CD86,CSF3,CXCL16,FAS,ICAM1, L1 18 & $140(16)$ \\
\hline ciproffloxacin & chemical drug & & 1,571 & & $1,98 E-05$ & 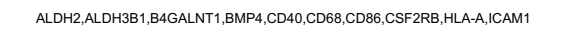 & $162(13)$ \\
\hline PTEN & phosphatase & & 0,065 & & $1,99 \mathrm{E}-05$ & 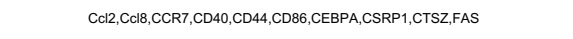 & $140(16)$ \\
\hline NFAT5 & transcription regulator & Activated & 2,355 & bias & $2,10 \mathrm{E}-05$ & 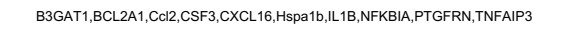 & $113(9)$ \\
\hline carnobyl iron & chemical reagent & & 1,960 & bias & $2,11 E-05$ & COL12,CEBPA,Hamp/Hamp2,L118 & \\
\hline Ferritin & complex & & & & $2,11 E-05$ & BMPG,ICAM1,LL1B,NFKBIA & $87(7)$ \\
\hline PSMB9 & peptidase & & 1,067 & bias & 2,11E-05 & HLA-A,NFKBIA,RELB,TNFAPP3 & $97(10)$ \\
\hline S-nitrosoglutathione & chemical toxicant & & $-0,873$ & & $2,26 E-05$ & BIRC3,CD86, CDBA, ICAM1,NFKBAA,NQ01,PRKG1 & $190(19)$ \\
\hline AHR & ligand-dependent nuclear receptor & & 0,638 & & $2,28 E-05$ & 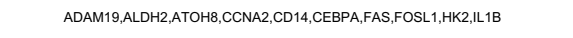 & $226(21)$ \\
\hline Hbb-b1 & transporter & & 1,408 & bias & $2,31 E-05$ & 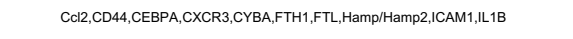 & $174(13)$ \\
\hline PGR & ligand-dependent nuclear receptor & & 1,985 & bias & $2,34 E-05$ & 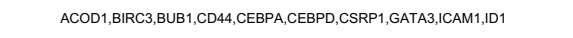 & $235(21)$ \\
\hline FGF2 & growth factor & Activated & 3,465 & bias & $2,38 E-05$ & AKR1B10,ANG,BMP4,CC12,CSF1,CSF3,CXCL12,ENPP1,FAS,FOSL1 & $206(25)$ \\
\hline butyric acid & chemical-endogenous mammalian & & 0,316 & & $2,53 \mathrm{E}-05$ & BCL2A1,CASP3,CCNA2,CD44,CD81,CD83, CD866,CSF1,FTH1,GATA3 & $245(21)$ \\
\hline Ifrar & group & Activated & 3,040 & bias & $2,53 E-05$ & 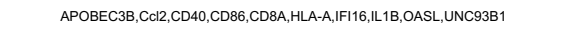 & $142(18)$ \\
\hline GPD1 & enzyme & & & & $2,53 \mathrm{E}-05$ & 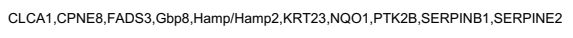 & \\
\hline PAEP & other & & $-0,277$ & bias & $2,57 E-05$ & CD83,СXCL16,CXCR3,GATA3,LL1RL1 & 128 (12) \\
\hline superoxide & chenical-endogenous mammalian & & 0,849 & bias & $2,57 E-05$ & СYBA,ICAM1,ID3,LL18,SOD2 & 194 (17) \\
\hline S100AB & other & & 0,737 & bias & $2,64 \mathrm{E}-05$ & BMF,COL2,CP, CSF2RB, CXCL14,FAS,FOSL1, GBB2,ICAM1,L118 & $145(14)$ \\
\hline trans-hydroxytamoxxien & chemical drug & & $-1,994$ & & $2,64 E-05$ & 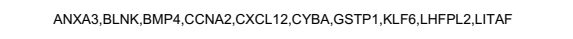 & $125(7)$ \\
\hline ARNT2 & transcription regulator & Activated & 3,860 & bias & $2,79 E-05$ & ANG,BST1, CD86, CYBA,FNBP 1, GALLTT4,GRM8,GSTA3, HLA-A,ICAM1 & \\
\hline emodin & chemical drug & & $-1,732$ & bias & $2,81 E-05$ & CCl2,CD86, CLDN4, ICAM1, LL18, IRF5,MRC1,SOD2 & 136 (18) \\
\hline втк & kinase & & 1,486 & & $2,99 \mathrm{E}-05$ & 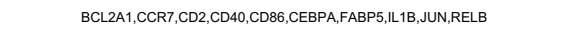 & $159(18)$ \\
\hline dinoprost & chemical-endogenous mammalian & & 0,213 & bias & $3,02 E-05$ & GSTP1,HMGCS2,ID1,ID, IL18, JUN,MAPKG,PTK2B,RUN×2 & 209 (23) \\
\hline PLG & peptidase & Activated & 2,883 & bias & $3,02 E-05$ & CD40,CSF1,DOCK1,LL18,ILLRL1,IQSEC1,MRC1,RALA,SERPINE1 & 158 (17) \\
\hline sox11 & transcription regulator & & 1,320 & bias & $3,05 E-05$ & 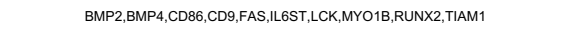 & $131(7)$ \\
\hline SLC25A13 & transporter & & & & 3,05E-05 & 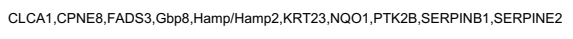 & \\
\hline$T \times N$ & enzyme & & 0,132 & & $3,10 E-05$ & APOA1,CD40,CD86, ICAM1, IL1B,NFKBIA,SOD2 & $127(16)$ \\
\hline isobutylmethyxanthine & chemical toxicant & Activated & 2,774 & bias & 3,12E-05 & ALDH3B 1, CC12,CD14,CD44,CEBPA,CEBPD,DHRS3,LL18,JUN,KLF4 & $222(21)$ \\
\hline Ap1 & complex & & 1,673 & bias & $3,25 E-05$ & 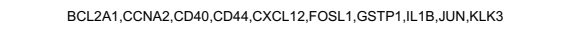 & $189(21)$ \\
\hline diethymaleate & chemical toxicant & & $-0,600$ & & $3,42 E-05$ & GSTP1,LL1B,NFKBIA,NQ01,TNFAIP3 & $182(16)$ \\
\hline GNPAT & enzyme & & & & $3,48 E-05$ & CС12,L1B, UGT8 & \\
\hline CBX5 & transcription regulator & Inhibited & $-3,317$ & bias & $3,51 \mathrm{E}-05$ & BCL11A,СD68,КLLK3,KRT20,LAMM3,РPROM2,RUNX2,SLC2A3,SYTL2,TM4SF1 & \\
\hline cholecalifierol & chemical-endogenous mammalian & & 1,548 & bias & 3.51E-05 & CD 14,CD40,CD83,,CD88,CEBPA,CEBPD,CSF1, JUN,LY75,RUN×2 & $198(23)$ \\
\hline
\end{tabular}




\begin{tabular}{|c|c|c|c|c|c|c|c|c|}
\hline IL5 & & cytokine & Activated & 4,130 & bias & $3,56 E-05$ & 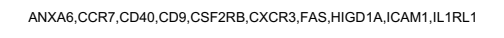 & $198(18)$ \\
\hline tamoxifen & & chemical drug & & 1,264 & & $3,56 \mathrm{E}-05$ & ANG,BMP2,BMP4,CAV2,CCNA2, CXCL12,DEPP1,GAP43,GDF 15,GJB2 & $228(24)$ \\
\hline C5AR1 & & G-protein coupled receptor & Activated & 2,778 & bias & $3,60 E-05$ & 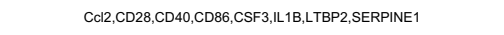 & $138(15)$ \\
\hline TNFSF14 & & cytokine & Activated & 2,180 & bias & $3,64 \mathrm{E}-05$ & BIRC3,CCI2,CD40,CD86, СXCL12,ICAM1 & $151(18)$ \\
\hline clodronic acid & & chemical drug & & 0,721 & bias & $3,64 \mathrm{E}-05$ & CC12,CD40,CD86, ICAM1,IL1B,MRC1 & $204(16)$ \\
\hline твк1 & & kinase & & $-0,149$ & bias & $3,65 E-05$ & 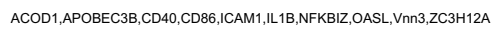 & $181(19)$ \\
\hline enterotoxin $B$ & & biologic drug & Activated & 2,759 & bias & $3,72 E-05$ & 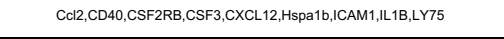 & $188(10)$ \\
\hline TEAD4 & & transcription regulator & Activated & 2,000 & bias & $3,72 E-05$ & ARIDSB, BMP4,FJX1, GATA3,JUN,NFKBZZ,NOTCH2,SLCZA3, TRNP1 & \\
\hline мMP9 & & peptidase & & 1,682 & bias & $3,72 E-05$ & CC12,CD44,CD68, CXCL12,GAP43,ICAM1,JAG1,SDC4,SERPINE2 & $215(20)$ \\
\hline NFKB2 & & transcription regulator & & 1,706 & bias & $3,72 E-05$ & 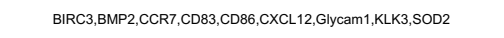 & $151(16)$ \\
\hline carbon monoxide & & chemical-endogenous mammalian & & $-0,980$ & bias & $4,06 E-05$ & CASP3,CD14,CXCL12,Hamp/Hamp2,ICAM1, I1 18,NQ01, SERPINE1 & $186(20)$ \\
\hline fingolimod & & chemical drug & Inhibited & $-2,876$ & bias & $4,11 E-05$ & CO12,CCR7,CD40,CD86,FOSLL, 1CAM1,LL18,RUNX2,SERPINE1 & $149(16)$ \\
\hline MRTFB & & transcription regulator & & 1,667 & bias & $4,16 E-05$ & 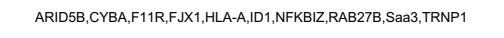 & \\
\hline Pam3-cys & & chemical toxicant & Activated & 2,493 & bias & $4,18 E-05$ & CD14,CD83, CD86, L118,LL1RL1,SERPINE1,SOD2 & $155(17)$ \\
\hline v0126 & & chemical -kinase inhibitor & Inhibited & $-4,053$ & bias & $4,30 \mathrm{E}-05$ & BIRC3,BMPG, CO12,CD44,CEBPA,CEBPD, CLDN4,CSF3,CXCL12,FOSL1 & $222(20)$ \\
\hline TNFSF10 & & cytokine & Activated & 2,397 & & 4,35E-05 & 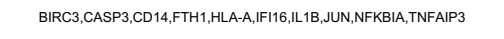 & $142(15)$ \\
\hline PPARGCIA & & transcription regulator & & $-1,278$ & & $4,39 \mathrm{E}-05$ & ALAS1,ANG,BMP4,CD40,СD68,CDC42EP1,CEBPD,CXCL14,GDF15, HK2 & 209 (19) \\
\hline UXT & & transcripition regulator & & $-0,865$ & bias & 4,40E-05 & B4GALT1,BUB1,CCNA2,KLK3,NFKBIA,TNFAIP3 & $137(7)$ \\
\hline actinomycin D & & biologic drug & & $-0,173$ & bias & 4,46E-05 & 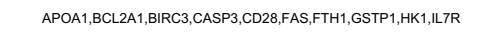 & 229 (19) \\
\hline w7 & & chemical reagent & & $-1,387$ & bias & 4,49E-05 & BRRC3,СD14,СD83,СD86,КடК3 & $81(7)$ \\
\hline zymosan A & & hemical - endogenous non-mammalia & Activated & 2,236 & bias & 4,49E-05 & $\mathrm{CCl2,CD40,CD86,FAS,IL1B}$ & $133(15)$ \\
\hline MAPK14 & & kinase & Activated & 2,652 & bias & 4,51E-05 & BMP2, СC2, СCNA2, СCR7, СD40, СD83, СD86, СEBPD,FAS, САM1 & 146 (17) \\
\hline IFN alphalbeta & & group & & 1,778 & bias & $4,54 \mathrm{E}-05$ & CCR7,CD40,CD83, CD886, СXCL14,CXCL16, HLA-A, IF116,MRC1 & $184(17)$ \\
\hline CPG ODN 1826 & & chemical reagent & Activated & 2,906 & bias & 4,54E-05 & Apol7e (includes others),CCR7,CD 14,CD40,CD83,CD86, CYBA,Ll18,PARP14 & $148(21)$ \\
\hline TP73 & & transcription regulator & & 1,612 & bias & $4,64 E-05$ & ADAM19,C1R,CASP3,CC12,CCNA2,CD83,,CLDN10,CLMN, DHRS3,FAS & $202(22)$ \\
\hline AR & & ligand-dependent nuclear receptor & Activated & 2,243 & bias & $4,68 \mathrm{E}-05$ & B4GALNT1,BASP1,BMF,,BMPA,BUB1, CAV2,CCNNA2, CXCL12,FABPF, GDF15 & $218(23)$ \\
\hline LTBR & & transmembrane receptor & Activated & 2,000 & bias & $4,82 E-05$ & BST1,CL12,CD866,CXCL12,GIycam1,1CAM1,NFKBIA & $88(10)$ \\
\hline KLF4 & 0,988 & transcription regulator & & 0,998 & bias & 4,86E-05 & ACOD1,ALCAM,CASP3, CD 14,CD44,CEBPA,,GJB2,HSPB 1, ID, KLLF4 & $204(18)$ \\
\hline mir-8 & & microRNA & Inhibited & $-2,578$ & bias & $5,11 E-05$ & BMP2,BST1, DDIT4L,FTH1,L118,KLF4,KRT19,LRP1,PERP,PTPN13 & \\
\hline PDCD1 & & phosphatase & & $-1,066$ & bias & $5,13 E-05$ & BATF,CCNA2,CD40,CD86,FAS, GZMA,,PADI2,PRKD3 & 151 (15) \\
\hline TNFSF13B & & cytokine & & 1,954 & bias & $5,13 E-05$ & BCL2A1,CCR7,CD40,CD83,CD86, ICAM1,JUN,RELB & $128(13)$ \\
\hline HLX & & transcription regulator & & $-0,663$ & bias & $5,28 E-05$ & BMP2,Cd24a,CD44,GDF 15,ICAM1,JUN & \\
\hline MMP14 & & peptidase & & $-0,816$ & & $5,28 E-05$ & CSF3,GAP43,JAG1, LRP1,Saa3,SDC1 & $32(3)$ \\
\hline GJA1 & & transporter & & $-0,883$ & & $5,28 E-05$ & ВMP2,BMP4,CD40,GJB2,ІСАM1,ІL1B & 159 (19) \\
\hline eplerenone & & chemical drug & Inhibited & $-2,418$ & bias & $5,28 E-05$ & COL2,CD68, CYBA, ICAM1, L1 18, SERPINE1 & $134(10)$ \\
\hline NRG1 & & growth factor & & 1,341 & bias & 5,33E-05 & 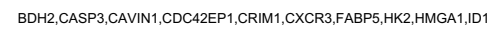 & $197(18)$ \\
\hline EPHB1 & & kinase & & 1,982 & bias & $5,36 E-05$ & CC12,LGST,JUN,SERPINE1 & $194(19)$ \\
\hline
\end{tabular}




\begin{tabular}{|c|c|c|c|c|c|c|c|c|}
\hline tyrphostin AG 1296 & & chemical -kinase inhibitor & & $-1,980$ & bias & $5,36 E-05$ & CO12,CD14,ICAM1,JUN & $187(17)$ \\
\hline EGFR & & kinase & & 1,885 & bias & $5,37 E-05$ & 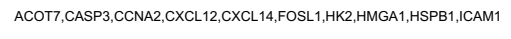 & 228 (22) \\
\hline methotrexate & & chemical drug & & $-0,377$ & & $5,44 E-05$ & C1R,CASP3,CD28,CFI,CSF1,CXCL 12,FAS, GAP43, GZMA,HMGCS2 & $188(22)$ \\
\hline ITGB2 & & transmembrane receptor & & 0,000 & & 5,54E-05 & 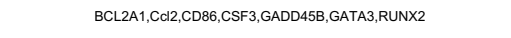 & $154(17)$ \\
\hline $\mathrm{c5}$ & & cytokine & Activated & 3,200 & bias & $5,56 E-05$ & 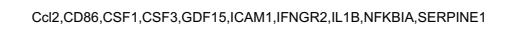 & $147(15)$ \\
\hline gentamicin & & chemical drug & Activated & 2,080 & & 5,71E-05 & 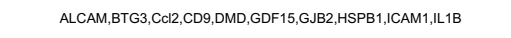 & $162(14)$ \\
\hline tributryin & & chemical drug & Activated & 2,157 & bias & 5,75E-05 & 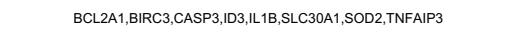 & \\
\hline CPG ODN 2395 & & chemical reagent & Activated & 2,236 & bias & $5,79 E-05$ & $\mathrm{Cl} 12, \mathrm{CD} 14, \mathrm{CD} 40, \mathrm{CD} 86, \mathrm{LL} 1 \mathrm{~B}$ & $141(15)$ \\
\hline CYP2J2 & & enzyme & & $-0,785$ & & $5,79 E-05$ & CCl2, CD 44,ICAM1,L118,NFKBIA & $127(17)$ \\
\hline fulvestrant & & chemical drug & & $-0,233$ & & $5,84 \mathrm{E}-05$ & APOA1,BMP2,BMP4,CAV2,CC12,CCNA2,CSF1,CXCL12,GJB2,,GSTP1 & $210(20)$ \\
\hline raloxifene & & chemical drug & & 0,033 & & $6,08 E-05$ & ANXA3,BLNK,BMP2,BMP4,CSF1,CXCL12,JUN,KLFF,LITAF,MYO1B & $221(22)$ \\
\hline PPARD & & ligand-dependent nuclear receptor & & 0,022 & bias & $6,26 E-05$ & 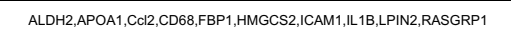 & $197(18)$ \\
\hline Nox4 & & enzyme & Activated & 2,425 & bias & $6,31 E-05$ & BMF,CCL2,CD44,ICAM1, Saa3,SERPINE 1 & $183(18)$ \\
\hline PAF1 & & other & Activated & 2,646 & bias & $6,35 E-05$ & BIRC3,KLF4,NFKBIZ,OASL,SDC4,SERPINE1,SOD2 & \\
\hline MAPK 7 & & kinase & Activated & 2,580 & bias & 6,35E-05 & BCL2A1,101,1L18,JUN,KLFF,NQO1,STEAP4 & $212(23)$ \\
\hline Марзк7 & & kinase & & 1,616 & bias & $6,42 E-05$ & BMP4,CASP3, CC12,LL18, JUN,NFKBZZ,NOD2,Vnn3 & $181(17)$ \\
\hline SMARCB1 & & transcription regulator & & 1,933 & bias & $6,46 \mathrm{E}-05$ & CCNA2,CD44,CSF 1, CYP3A7,DOCK4,F11R,FAS,FBP1,GJB2,F|F116 & $193(11)$ \\
\hline mycophenolic acid & & chemical drug & Activated & 2,121 & bias & $6,67 E-05$ & 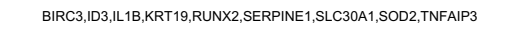 & $142(14)$ \\
\hline docosahexaenoic acid & & chemical drug & & $-0,367$ & bias & $6,85 E-05$ & 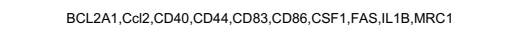 & $143(15)$ \\
\hline IRF2 & & transcription regulator & Activated & 2,277 & bias & $6,91 E-05$ & 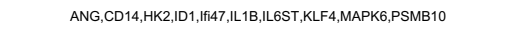 & $156(18)$ \\
\hline Gcg & & other & & 0,707 & bias & $7,16 E-05$ & ANG,ARX,FABPF, FBP1, Hamp/Hamp2, OAT, RNASE4,SDC1 & \\
\hline TCF7L2 & & transcription regulator & Activated & 2,299 & bias & $7,17 E-05$ & ВMP4,СSRP1,СYP3A7,DOCK10,FBP1,FTH1,GAB1,HMGCS2,ID1,ID3 & $138(7)$ \\
\hline NR1H3 & & ligand-dependent nuclear receptor & & $-1,309$ & & $7,29 E-05$ & APOA1,CC12, CC18,CCNA2,,CCR7,CD40,CSF3,СXCL16,FBP1,,GSTP1 & $193(15)$ \\
\hline LYN & 0,712 & kinase & & 1,177 & bias & $7,31 E-05$ & 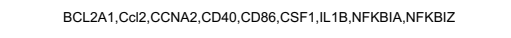 & $178(15)$ \\
\hline L-buthionine (SR)-sulfoximine & & chemical drug & & 0,000 & bias & $7,36 E-05$ & CASP3,NFKBAA,NQ01,SERPINE1,TNFAP3 & $165(11)$ \\
\hline lactacystin & & chemical - protease inhibitior & & 0,320 & bias & $7,45 E-05$ & CO12,CD9,FAS,FBP1,FOSL1,FLL,GATA3,,GSTP1, HLA-A,HSPB1 & $188(20)$ \\
\hline KRIT1 & & other & & 0,000 & bias & $7,90 \mathrm{E}-05$ & BMP2,JUN,KLF4,SOD2 & \\
\hline PIК $3 C A$ & & kinase & & 1,941 & & $7,97 \mathrm{E}-05$ & ALCAM, CC12,CD14,Cd24a,CD44,CEBPD,JAG1,SERPINE1 & $211(23)$ \\
\hline PLAU & & peptidase & & 0,186 & bias & $7,97 E-05$ & ANG, CC12,GDF15,ICAM1,LL18, RELB,SERPINB 1,SERPINE1 & $232(24)$ \\
\hline CCL2 & & cytokine & & 1,401 & bias & $8,01 E-05$ & 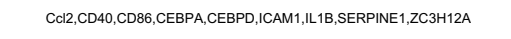 & 159 (17) \\
\hline FADD & & other & & 0,333 & bias & $8,01 E-05$ & BIRC3,CCI2,FAS, GADD45B,ICAM1,IRF5,JUN,KLFG,TNFAIP3 & $146(18)$ \\
\hline BRD2 & & kinase & & & & $8,26 E-05$ & 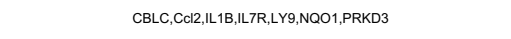 & \\
\hline SREBF1 & & transcription regulator & Activated & 2,466 & bias & $8,34 E-05$ & 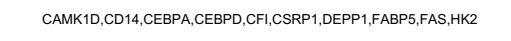 & $186(20)$ \\
\hline TMPRSSG & & peptidase & & & & $8,58 E-05$ & BMP6,Hamp/Hamp2,101 & \\
\hline Widc17 & & other & & & & $8,58 E-05$ & CO12,L1B,MRC1 & \\
\hline IKBKE & & kinase & & 0,594 & bias & $8,76 E-05$ & 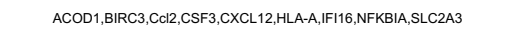 & 175 (15) \\
\hline RARG & & ligand-dependent nuclear r ecepptor & & 1,230 & & $8,76 E-05$ & 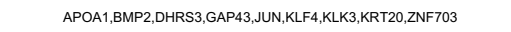 & $160(10)$ \\
\hline
\end{tabular}




\begin{tabular}{|c|c|c|c|c|c|c|c|}
\hline FASLG & cyokine & & 0,692 & & 8,82E-05 & CASP3,CD44,FAS,FTH1,L18,RUNX2 & $187(18)$ \\
\hline PF4 & cytokine & Activated & 2,401 & bias & $8,82 E-05$ & CD83,CD86, ICAM1,L181, KLF 4,LY775 & $137(18)$ \\
\hline NGF & growth factor & Activated & 2,488 & bias & $8,90 \mathrm{E}-05$ & AKR1B10, CCR7, CD40,CDB3, CD86,FAS,FOSL1, GAP43,ID1,JUN & $149(18)$ \\
\hline inosine & chemical-endogenous mammalian & Activated & 2,646 & bias & $9,38 E-05$ & ALCAM,ANTXR2,CD68,FTL,GAP43,SERPINB1,SERPING1 & \\
\hline carrageenan & chemical drug & Activated & 2,592 & bias & $9,38 E-05$ & CO12,FOSL1,GRMB, ICAM1,L18, JUN,NFKBBA & $205(18)$ \\
\hline ZFP36 & transcription regulator & Inhibited & $-2,415$ & bias & $9,57 E-05$ & BIRC3,FOSL 1, ICAM1, IL1B,JUN,LATS2,NEK2,NFKBIA,PNRC1 & $181(17)$ \\
\hline tert-butyl-hydroquinone & chemical reagent & Activated & 2,740 & bias & $9,57 E-05$ & FOSL1,FTH1,FTL,GSTA3,GSTP1,JUN,NQ01, SERPINE1,UGT 1A7 (includes others) & $186(19)$ \\
\hline 17-alpha-ethinyyestradiol & chemical drug & & 1,863 & bias & $9,75 E-05$ & CO12,CCR7,CD68, CXCR3, GDF 15,1F116,JUN,KRT 19,MRC1,PERP & $247(20)$ \\
\hline sүk & kinase & & $-0,256$ & & $9,80 \mathrm{E}-05$ & BIRC3,СCR7,CDA4,DOCK10,FOSL1, ,GATA3,ICAM1,1L18, LY9,RASSF4 & $192(22)$ \\
\hline wortmannin & chemical -kinase inhibitor & Inhibited & $-2,689$ & bias & $1,02 E-04$ & 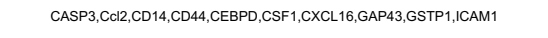 & $206(20)$ \\
\hline SAA & group & Activated & 2,403 & bias & $1,03 E-04$ & CCl2,CSF1,CSF3,ICAM1,IL18,MRC1 & $158(18)$ \\
\hline NORAD & other & Activated & 2,449 & bias & $1,03 E-04$ & BASP1,GADD45B, ID3,JUN,SDC1,TNFRSF12A & $93(7)$ \\
\hline Ins1 & other & & 1,372 & bias & 1,04E-04 & ALAS1,APOA 1,CEBPA,CEBPD, GATA3,GSTP1,HK2,L1 18,LLGST,JUN & 233 (23) \\
\hline hemin & chemical - endogenous mammalian & & 0,838 & & $1,04 E-04$ & 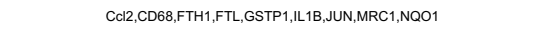 & $220(19)$ \\
\hline KITLG & growth factor & & 1,344 & bias & $1,08 E-04$ & 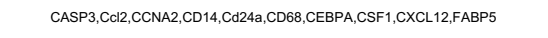 & $142(16)$ \\
\hline Iysophosphatidylcholine & chemical - other & & 1,692 & bias & $1,09 E-04$ & 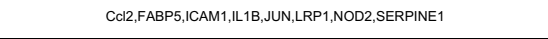 & $202(20)$ \\
\hline Bay 11-7082 & chemical -kinase inhibitor & Inhibitied & $-2,789$ & bias & $1,09 E-04$ & 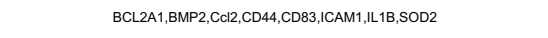 & $141(15)$ \\
\hline PLA262D & enzyme & & $-1,000$ & bias & 1,12E-04 & COL2, CD68, IL1B,MRC1 & $190(12)$ \\
\hline BCR (complex) & complex & & 1,354 & bias & 1,12E-04 & B4GALT1,BCL2A1,CASP3,,CCNA2,CCR7,CD44,CD86,FAS,KLF4,LCK & $140(16)$ \\
\hline JUNB & transcription regulator & & 1,109 & bias & 1,12E-04 & 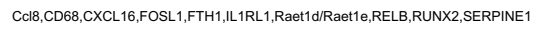 & ${ }_{133(13)}$ \\
\hline APC & enzyme & Inhibited & $-2,121$ & bias & $1,12 E-04$ & 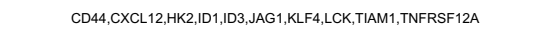 & $130(7)$ \\
\hline TCF & group & & & & $1,14 \mathrm{E}-04$ & BMP4,CDA4,ID3,KLKK3,PLS3, RCN1,SERPINE1,SFN, TSPAN8 & $134(7)$ \\
\hline แ36в & cytokine & & & & $1,15 E-04$ & CD40,CD83, СD86, LL1B,NFKBIZ & $130(13)$ \\
\hline BMPER & other & & $-1,432$ & bias & $1,15 E-04$ & BMP4,Hamp/Hamp2, ICAM1,ID1,RUNX2 & $15(4)$ \\
\hline ANGPT2 & growth factor & Activated & 3,384 & bias & $1,19 E-04$ & 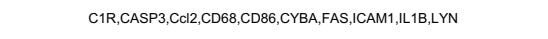 & $160(19)$ \\
\hline $\mathrm{NKX} \times-1$ & transcription regulator & & & & $1,20 \mathrm{E}-04$ & CTSZ,KLK3,PTPAA2,PTPRK,SDC1,SDC4,Vnn3 & \\
\hline NFKBIB & transcription regulator & & $-0,391$ & bias & $1,20 \mathrm{E}-04$ & BCL2A1, СD40,CD86, ICAM1,LL18,NFKBIA,Saa 3 & $117(12)$ \\
\hline ILRR & transmembrane receptor & & 0,447 & & $1,20 \mathrm{E}-04$ & CO12,CD14,CLCA1, FAS, GATA3, I11, RUN $\times 2$ & $157(17)$ \\
\hline carbonyl cyanide m-chlorophenyl hydrazone & chemical toxicant & & 1,890 & bias & $1,20 \mathrm{E}-04$ & BMPG,GDF 15,HK2, LAMB3,NFKBIZ,RND3,SERPINE1 & $108(7)$ \\
\hline TLR5 & transmembrane receptor & & 1,964 & bias & $1,21 E-04$ & 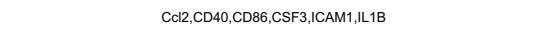 & $143(15)$ \\
\hline thalidomide & chemical drug & Inhibited & $-2,186$ & bias & $1,21 E-04$ & BIRC3,GADD45B, GDF 15, ICAM1, I1 18,SERPINE1 & $165(19)$ \\
\hline RAC1 & enzyme & Activated & 2,184 & bias & $1,23 E-04$ & 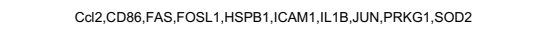 & $182(19)$ \\
\hline GNA15 & enzyme & & & & $1,23 E-04$ & BMP4,CAV2, CCL12,CEBPD,CP,ENPP1,FOSL1,,PDLIM1,,SOD2,XDH & $169(11)$ \\
\hline GW9662 & chemical reagent & Inhibitied & $-2,183$ & bias & $1,24 \mathrm{E}-04$ & 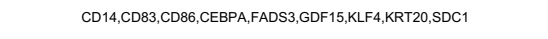 & $167(11)$ \\
\hline 10E,12Z-octadecadienoic acid & chemical- endogenous mammalian & & 0,921 & & $1,24 E-04$ & 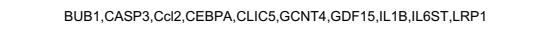 & $162(20)$ \\
\hline SPP1 & cytokine & Activated & 2,414 & bias & $1,28 E-04$ & 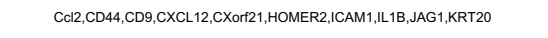 & $200(21)$ \\
\hline swow1 & transporter & Activated & 2,525 & bias & $1,28 E-04$ & 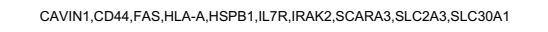 & \\
\hline
\end{tabular}




\begin{tabular}{|c|c|c|c|c|c|c|c|}
\hline BMP7 & growth factor & & 1,681 & bias & $1,28 \mathrm{E}-04$ & 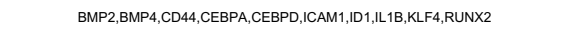 & 157 (19) \\
\hline triamcinolone acetonide & chemical drug & & 0,997 & & $1,32 E-04$ & B4GALNT1,BCLLA1,BMP2,BMPA,BMPG, CD44, GZMA,LLGST,JUN,NFKBIA & $117(7)$ \\
\hline heparin & chemical - endogenous mammalian & Activated & 2,180 & bias & $1,32 E-04$ & ALAS1,CCD40, CD83, CD886, Hamp/Hamp2, ICAM1 1,L6ST, RUN ×2 & 139 (16) \\
\hline SRF & transcription regulator & & 1,598 & bias & $1,33 E-04$ & BATF,BMP2,Cald1, CEBPD, CSRP 1,DMD,FOSL1,ITGA9,JUN,LRP1 & $29(3)$ \\
\hline colchicine & chemical drug & Activated & 2,271 & bias & $1,35 E-04$ & CD40,FAS,GAP43,IL 18,JUN,NQO1,TMEM123 & 208 (19) \\
\hline n-nitrosomethylbenzylamine & chemical toxicant & & & & $1,37 E-04$ & 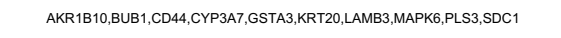 & \\
\hline CARM1 & transcription regulator & Activated & 2,219 & bias & $1,40 \mathrm{E}-04$ & 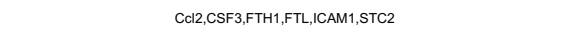 & $178(14)$ \\
\hline fluvastatin & chemical drug & & $-0,059$ & & 1,40E-04 & CD40,CD81, CD9, CYBA, LRP 1, SERPINE 1 & \\
\hline pristane & chemical toxicant & & & & $1,40 \mathrm{E}-04$ & CO12,CD86, CXCR3, F116, I1 18,IRF5 & $145(14)$ \\
\hline сомMD1 & transporter & & $-1,732$ & bias & $1,41 E-04$ & BIRC3,HK2,KRT19,NFKBIA,STC2 & $149(7)$ \\
\hline heme & chemical-endogenous manmmalian & & 0,659 & bias & $1,41 E-04$ & ALAS1,CD14,CD86, ICAM1,MRC1 & $122(10)$ \\
\hline calcimycin & chemical reagent & Activated & 2,658 & bias & $1,42 E-04$ & 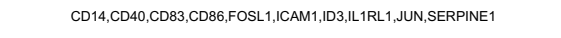 & $194(21)$ \\
\hline ionomycin & chemical reagent & Activated & 3,524 & bias & 1,43E-04 & 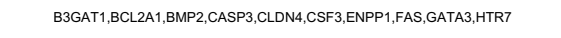 & $153(15)$ \\
\hline $\sin 1$ & transcription regulator & Activated & 3,729 & bias & $1,46 E-04$ & ANG,BST1,CD86, CYBA,FNBP1, GALLT4, GRM8, GSTA3, HLA-A,ICAMM1 & \\
\hline EHF & transcription regulator & & 1,480 & bias & $1,46 E-04$ & BLNK,BMP4,CASP3,GPR68,JAG1,KLK3,LYN,NOTCH2,SERPINE1 & \\
\hline trinitrobenzenesulfonic acid & chemical reagent & Activated & 2,904 & bias & $1,46 E-04$ & 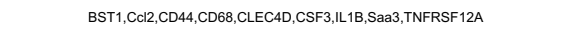 & 198 (15) \\
\hline eicosapentenoic acid & chemical drug & & $-1,089$ & & $1,46 \mathrm{E}-04$ & CD40,CD44,CD83, СD86,CEBPD, L118,JUN,MRC1,SERPINE1 & $185(19)$ \\
\hline bortezomib & chemical drug & & $-0,498$ & & $1,50 \mathrm{E}-04$ & BCL2A1,BIRC3,CCL12,CSF2RB,CXCL12,DMD,FAS, GADD45B, GDF 15, Hspa1b & $193(17)$ \\
\hline CPG ODN 1668 & chemical reagent & Activated & 2,578 & bias & $1,51 \mathrm{E}-04$ & 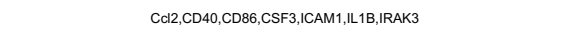 & 148 (17) \\
\hline fenofibrate & chemical drug & & $-0,273$ & bias & $1,56 \mathrm{E}-04$ & APOA 1, CC12,CDA4, CD68, FBP 1, GSTA3,ICAM1, LLST,JUN,MEST & $166(17)$ \\
\hline PPIF & enzyme & & 0,522 & & $1,59 E-04$ & 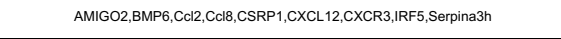 & $123(7)$ \\
\hline ADAM12 & peptidase & & $-1,425$ & bias & $1,60 \mathrm{E}-04$ & 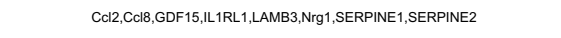 & \\
\hline Cdc42 & enzyme & & $-1,400$ & bias & $1,62 E-04$ & COI2,CD86, ,CEBPA,CSFF 1, CTSZZ,FAS & $173(18)$ \\
\hline sox2 & transcription regulator & & 1,372 & bias & $1,64 E-04$ & ACOD1,BMP4,CD44, CD9, СXCL14,DOCK10,GAB1, GADD45B, GATA3,ID3 & \\
\hline IFNA2 & cytokine & Activated & 2,473 & bias & $1,64 E-04$ & C1R,CD28,CD86, СORO2A,CXCR3,ENPP1,FAS, HLA-A,IF16, IL1B & $169(18)$ \\
\hline coumarin & chemical drug & & & & $1,69 \mathrm{E}-04$ & GSTA3,GSTP1,NQ01 & \\
\hline MAPKAPK2 2 & kinase & & 1,741 & bias & $1,70 \mathrm{E}-04$ & CC12,CEBPD, HSPB1,ICAM1,L118,MRC1,NFKB|A & 208 (19) \\
\hline mir-183 & microRNA & Inhibited & $-2,219$ & bias & $1,72 E-04$ & JUP,KLF4,LRP1,SERPINE2,SLC30A1 & \\
\hline arsenic & chemical toxicant & & 0,796 & bias & $1,72 E-04$ & BIRC3,CCR7,FTH1,FTL,NQO1 & $170(15)$ \\
\hline CREB1 & transcription regulator & Activated & 2,198 & bias & $1,72 E-04$ & ALAS1,CAMK1D,CAMK4,CCNA2,CD688,CD9, CEBPD,,CRIM1,ESD,,GADD45B & $179(13)$ \\
\hline oleic acid & chemical- endogenous mammalian & & $-0,748$ & bias & $1,76 \mathrm{E}-04$ & 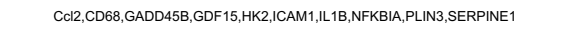 & 198 (19) \\
\hline TLR7 & transmembrane receptor & Activated & 3,532 & bias & $1,86 E-04$ & 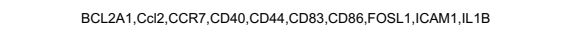 & $132(14)$ \\
\hline carbon tetrachloride & chemical toxicant & Activated & 3,134 & bias & $1,86 \mathrm{E}-04$ & 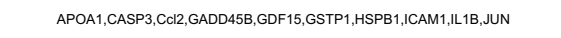 & $194(17)$ \\
\hline F7 & peptidase & & $-0,291$ & bias & $1,87 \mathrm{E}-04$ & CA12,FOSL1,ICAM1,IL18,JAG1,RND3 & $191(21)$ \\
\hline 2-amino-5-phosphonovaleric acid & chemical - other & & $-0,971$ & & $1,88 \mathrm{E}-04$ & 2610035D17RRK,ADAM19,BTG3,CGN,GADD45B,JUN,LLFPLL2,LURAP1,NHSL1,RELB & \\
\hline cervivastatin & chemical drug & & $-0,519$ & & $1,93 \mathrm{E}-04$ & APOA1,BMP2,CD40,HSPB 1,ICAM1,JUN,MAL,SERPINE1 & 148 (17) \\
\hline $\mathrm{NKX2-3}$ & transcription regulator & & $-1,059$ & & $1,95 E-04$ & 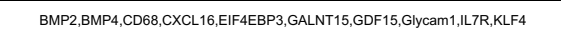 & \\
\hline
\end{tabular}




\begin{tabular}{|c|c|c|c|c|c|c|c|}
\hline EIF_AK 2 & kinase & Activated & 2,124 & bias & $2,01 E-04$ & BCL2A1,CEBPD,FAS,Hspa 16,LL18,NFKBIA,PSMB 10,SOD2,,TNFAPP3 & 159 (13) \\
\hline ETS1 & transcription regulator & Activated & 2,554 & bias & $2,05 E-04$ & 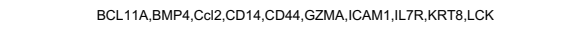 & $225(21)$ \\
\hline BMPR2 & kinase & & 1,984 & bias & $2,07 E-04$ & CXCL12,Hamp/Hamp2,ICAM1,ID1,RUN×2 & $187(18)$ \\
\hline TRIB3 & kinase & & $-0,555$ & bias & $2,07 E-04$ & CEBPA,GDF15,ID1,RUN $\times 2$, STC2 & $134(7)$ \\
\hline nystatin & chemical drug & & 1,982 & bias & $2,07 E-04$ & CD40, СD86, ICAM, L1, 1B & $155(19)$ \\
\hline CHRNA 1 & transmembrane receptor & & $-0,577$ & bias & $2,07 E-04$ & CD28,CD40,CD86,FAS & $136(11)$ \\
\hline NRP1 & transmembrane receptor & Inhibited & $-2,000$ & & $2,07 E-04$ & $\mathrm{CD} 40, \mathrm{CD} 86, \mathrm{ID} 1, \mathrm{L1} 1 \mathrm{~B}$ & $143(18)$ \\
\hline แ11 & cytokine & & 0,447 & & $2,11 \mathrm{E}-04$ & BCL2A1, CD40, CEBPD, Clec2d (includes others),ICAM1,L1B, IL6ST & $181(19)$ \\
\hline POLR2A & enzyme & & & & $2,11 E-04$ & BMF,CCNA2,KLKK3,NFKBIA,STC2, TNFAIP3,YYAP1 & \\
\hline Acox 1 & enzyme & & $-0,632$ & & $2,12 E-04$ & CXCL12,GSTP1, KLF6, KRT 23,,KRT8,MGLL,OAT,Saа3,SLCAAA, UGT8 & $114(7)$ \\
\hline CCR5 & G-protein coupled receptor & & 1,211 & bias & $2,14 \mathrm{E}-04$ & 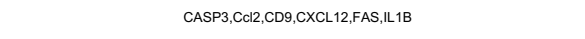 & $184(18)$ \\
\hline MAP2K1 & kinase & Activated & 2,412 & bias & $2,16 \mathrm{E}-04$ & BCL2A1, BIRC3,,CCNA2, CD44,CEBPA,CRIM1,FOSL1, JUN,JUP,NFKBIA & $190(20)$ \\
\hline LEP & growth factor & Activated & 2,555 & bias & $2,17 \mathrm{E}-04$ & APOA $1, \mathrm{BCL} \angle \mathrm{AA}, \mathrm{CASP} 3, \mathrm{CL1} 2, \mathrm{CCNA} 2, \mathrm{CD} 14, \mathrm{CD} 40, \mathrm{CD} 68,, \mathrm{CSF} 3, \mathrm{CYBA}$ & $155(18)$ \\
\hline PLA2G10 & enzyme & & $-1,134$ & bias & $2,17 \mathrm{E}-04$ & BMP4,CD40, CD8A,DHRS3,FAS,GATA3,LL18,KRTR,MRC1 & $238(20)$ \\
\hline S100A9 & other & & 1,263 & bias & $2,20 \mathrm{E}-04$ & 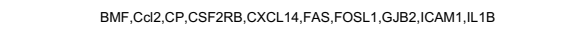 & $161(17)$ \\
\hline testosterone & chemical- endogenous mammalian & & 0,822 & bias & $2,28 E-04$ & 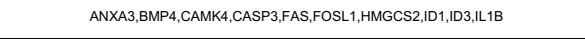 & $225(21)$ \\
\hline TRIM24 & transcription regulator & Inhibitied & $-2,828$ & bias & $2,34 E-04$ & BLNK,CSRP1,Iff47,IFNGR2,MOV10,MS4Aab (includes others), OASL,PSMB 10,SERPINE1 & \\
\hline TAZ & enzyme & & & & $2,34 E-04$ & AHNAK2,BASP1,CAV2, CD44,FF16,KLFG, RUN 22, SERPINE1,UPP1 & \\
\hline CXCR4 & G-protein coupled receptor & & 1,342 & bias & 2,35E-04 & CD44,CD9, CXCL12,ID1,RRAS,RUNX2,ZBTB16 & $175(22)$ \\
\hline mir-30 & microRNA & Inhibitied & $-2,397$ & bias & $2,44 E-04$ & ARIDSB, COL2,L1B,RUNX2,SERPINE1,UGT8 & $132(12)$ \\
\hline IFIH1 & enzyme & Activated & 2,433 & bias & $2,44 E-04$ & Clec2d (includes others),FAS, I1 18,LCK,NFKBA,RND3 & $134(13)$ \\
\hline HIF $F$ A & transcription regulator & Activated & 2,805 & bias & $2,46 E-04$ & 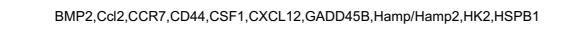 & $181(15)$ \\
\hline GNAQ & enzyme & & & & $2,47 E-04$ & ANXA3,BMP2,,BMP4,CC12,CDA4,CXCL 12,HIGD 1A,HK2,SERPINE1,YAP1 & $180(16)$ \\
\hline NCF1 & enzyme & & 1,982 & bias & $2,48 E-04$ & CC12,CD14,CD44,ICAM1,SERPINE1 & $152(17)$ \\
\hline САMP & other & & 0,959 & bias & $2,53 E-04$ & 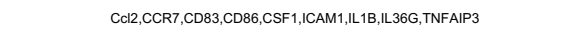 & 150 (18) \\
\hline muc1 & other & & 0,570 & bias & $2,611-04$ & CD40,CD83,CD86,MRC1,NFKBIA,OASL,SOD2 & $182(18)$ \\
\hline N-acetylmuramyl-L-alany-D-isoglutamine & hemical - endogenous non-mammalia & & 1,975 & bias & $2,611-04$ & Apol7 (includes others),CD40,CD86, ICAM1,L118,NFKBBA,NOD2 & $146(16)$ \\
\hline Histone h4 & group & & & & $2,70 \mathrm{E}-04$ & BTG3,CD40,DUSP16,GSTP1,/CAM1,LL18,MCM3,NEURL1,PREX1,RASGRP1 & $133(7)$ \\
\hline NR1H2 & ligand-dependent nuclear recepptor & & $-1,942$ & & $2,72 \mathrm{E}-04$ & CCI2,CCR7,CSF3,CXCL16,HK1,LL18,MRC1,NFKBA,SSaa 3 & $147(16)$ \\
\hline Collagen type II & complex & & $-0,728$ & & $2,78 \mathrm{E}-04$ & BMP2,BMP4,BMP6,CC12,IL1B,RRAS & 159 (17) \\
\hline IFNLR1 & transmembrane receptor & & & & $2,78 E-04$ & CLECAD, CSF1, L1 18, OASL,SOD2, TNFAP3 & \\
\hline diethylstilbestrol & chemical drug & & 1,253 & bias & $2,81 E-04$ & CCNA2,CD 14,CD83,CXCL14,FAS, GAP43, GJB2,HLA-A,KLF4,KLKK3 & $116(8)$ \\
\hline TGM2 & enzyme & Activated & 2,496 & bias & $2,83 E-04$ & BCL2A1,BRC3,СD86,CDCA7,CSF3,FERMT1,1L18, OASL,PARP 14,PLEKHO2 & $183(18)$ \\
\hline FIRRE & other & & & & $2,91 E-04$ & CSF1,СРF3,L1B & \\
\hline HJV & other & & & & $2,91 E-04$ & ВMP, Hamp/Hamp2, ID1 & $36(3)$ \\
\hline CTSE & peptidase & & & & $2,91 E-04$ & CD40,CD86,LL1B & $146(11)$ \\
\hline rabeprazole & chemical drug & & & & $2,91 E-04$ & CCl2,ICAM1,IL1B & \\
\hline
\end{tabular}




\begin{tabular}{|c|c|c|c|c|c|c|c|c|}
\hline vinpocetine & & chemical drug & & & & $2,91 \mathrm{E}-04$ & CCl2,ICAM1,L1B & 139 (9) \\
\hline BRAF & & kinase & & & & $2,93 \mathrm{E}-04$ & BIRC3,BMF,CCL2, CD40,CD86, CSF1,L1 18,RND3,SERPIINE2 & $144(16$ \\
\hline taurocholic acid & & chemical - endogenous mammalian & & 0,391 & & $2,95 E-04$ & 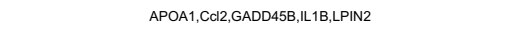 & $154(177$ \\
\hline mir-135 & & microRNA & Inhibited & $-2,183$ & bias & $2,95 E-04$ & CClB, CXCL12,IL18,KLF4,RUNX2 & \\
\hline vitamin K3 & & chemical drug & Activated & 2,236 & bias & 2,95E-04 & BMP6,СASP3,FAS, HK2,KLF6 & $166(14$ \\
\hline RUN $\times 3$ & & transcription regulator & Inhibited & $-3,000$ & bias & $3,00 E-04$ & CCR7,CRIM1,LL18,ILGST,JAG1, LLRRB,PXDN,RTN1,SCARA3,STC2 & 155 (7) \\
\hline Nos3 & & enzyme & & $-1,082$ & & $3,15 E-04$ & ВMP2,CC12,CD86, СXCL12,GSTP1,1СAM1 & $193(20)$ \\
\hline GPX1 & & enzyme & Inhibited & $-2,189$ & bias & 3,15E-04 & COI2,CD14,FAS, Hspa 1b,ICAM1,SOD2 & $150(18$ \\
\hline Fcer1 & & complex & & 1,802 & bias & $3,19 E-04$ & BCL2A1,CCL1,CDB3,CSF 1,L11,JUN,MRC1 & 160 (177 \\
\hline ॥25 & & cytokine & & 1,608 & bias & 3,19E-04 & CCl2,CSF3,F11R,GATA3,L18,LLRLL1,JAG1 & $179(17$ \\
\hline $\mathrm{CSF} 3$ & 1,184 & cytokine & & 1,1466 & bias & $3,20 \mathrm{E}-04$ & 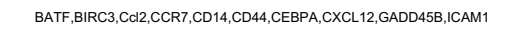 & $141(15$ \\
\hline reactive oxygen species & & chemical toxicant & & 1,824 & bias & $3,20 \mathrm{E}-04$ & 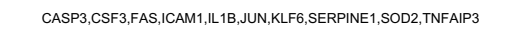 & $205(18$ \\
\hline cardiotoxin & & chemical - other & Activated & 2,828 & bias & 3,21E-04 & 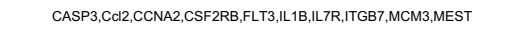 & \\
\hline geldanamycin & & hemical- endogenous non-mammalia & Inhibited & $-2,724$ & bias & $3,21 E-04$ & CD40,CEBPD,GPR 179, Hspa 16,ILLRLL 1,RFF,JUN,LCK,RNASELL,RTN1 & 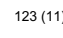 \\
\hline TRAF2 & & enzyme & & 0,822 & & $3,25 \mathrm{E}-04$ & BIRC3,FAS,ICAM1,Muc1, PTPN14,RHBDF1,Serpina3g (includes others), TNFAP3 & $100(12$ \\
\hline zinc & & chemical drug & & $-0,167$ & & $3,25 E-04$ & 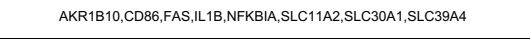 & 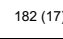 \\
\hline 1122 & & cytokine & & 1,334 & bias & $3,41 E-04$ & 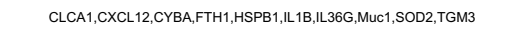 & $154(12$ \\
\hline tanespimycin & & chemical drug & & $-1,770$ & bias & $3,41 \mathrm{E}-04$ & BCL2A1,BRC3,FAS, FLT3,JUN,KRT, LYNN,SERPINE1,SOD2, TNFAPP3 & $132(17$ \\
\hline medroxyprogesterone acetate & & chemical drug & & $-1,019$ & & $3,41 E-04$ & 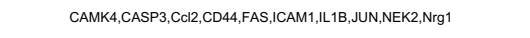 & $218(25) \mathrm{r}$ \\
\hline PTX3 & & other & & $-1,571$ & bias & 3,48E-04 & COl2, CCI8, GATA3, IL18,JUN & $147(16)$ \\
\hline ACSL4 & & enzyme & & $-1,342$ & bias & $3,48 E-04$ & CAMK1D,ENPP1,ERRMT1,PRKG1,RHOU & \\
\hline vinblastine & & chemical drug & Activated & 2,213 & bias & 3,48E-04 & CD40,CD86, HLA-A,LL1B,SOD2 & $142(177$ \\
\hline суре223 & & enzyme & & $-0,883$ & bias & $3,50 \mathrm{E}-04$ & 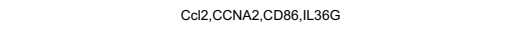 & \\
\hline PPP2R5C & & other & Inhibited & $-2,000$ & bias & $3,50 \mathrm{E}-04$ & BCL2A1,BRC3,RELB,TNFAPP 3 & $117(12$ \\
\hline P2RY2 & & G-protein coupled receptor & & & & $3,50 \mathrm{E}-04$ & COC12,GAP43,ICAM1,SERPINE1 & $142(17$ \\
\hline DAXX & & transcription regulator & & $-1,982$ & bias & $3,50 \mathrm{E}-04$ & CASP3,HSPB1,КLK3,SERPINE 1 & $208(19)$ \\
\hline SPIB & & transcription regulator & Activated & 2,813 & bias & $3,52 \mathrm{E}-04$ & 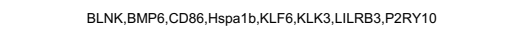 & \\
\hline HMGB1 & & transcription regulator & & 1,963 & bias & $3,52 \mathrm{E}-04$ & 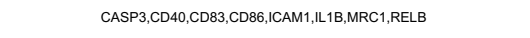 & $161(19$ \\
\hline SMAD1 & & transcription regulator & Activated & 2,429 & bias & $3,56 \mathrm{E}-04$ & CSF1,GADD458,Hamp/Hamp2,ICAM1,ID1,RUN×2 & $158(13$ \\
\hline PROC & & peptidase & & $-0,294$ & bias & $3,56 \mathrm{E}-04$ & BCL2A1, CCL2,ICAM1,LL18,SERPINE1,TNFAIP3 & $154(16)$ \\
\hline stallimycin & & biologic drug & Activated & 2,449 & bias & $3,56 \mathrm{E}-04$ & CSF1,IF161,IL1B,IL36G,IL7R,SP110 & $97(7)$ \\
\hline Nrith & & group & Inhibited & $-2,723$ & bias & 3,59E-04 & 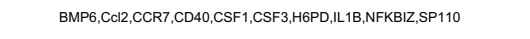 & $169(19$ \\
\hline SNAl1 & & transcription regulator & & 0,896 & & $3,79 E-04$ & BMP4,CGN,CLDN4,FOSL1,GDF15,ID1,KRT8,LASP1,RUN×2,SDC1 & $226(22$ \\
\hline dimethyl sulfoxide & & chemical drug & Activated & 2,578 & bias & $3,86 \mathrm{E}-04$ & BMP2,BMP4,IF16,LLL18,JUN,PTK2B,RUNX2,SLC11A2, TCf7,TPm1 & $206(22$ \\
\hline Go 6976 & & chemical -kinase inhibitor & Inhibited & $-2,607$ & bias & $3,87 E-04$ & ВMP2,BMP,,CL12,СD83,СSF3,NFКBIA,SOD2 & $144(16)$ \\
\hline МЕT & & kinase & & 0.633 & bias & $3,89 E-04$ & BMF,CD44,Hspa 16,HSPB 1, IL18,JUN,KLF4,NFKBIZ,SOD2 & $173(18$ \\
\hline Creb & & group & & 1,929 & bias & 3,91E-04 & ANTXR1,ANTXR2,BMPG,CD14,CEBPA,CEBPD, CLLN,CSRP1,FAS,FOSL1 & $159(11$ \\
\hline
\end{tabular}




\begin{tabular}{|c|c|c|c|c|c|c|c|}
\hline obeticholic acid & chemical drug & & $-1,347$ & & 4,01E-04 & APOA1,CL12,СD68,CEBPA,ICAM1,L18 & $144(17)$ \\
\hline NGFR & transmembrane receptor & & 0,895 & bias & $4,07 E-04$ & CCNA2,DMD,ILIRL1,JUN,SERPINE1 & $162(12)$ \\
\hline TNFSF 15 & cytokine & Activated & 2,200 & bias & $4,07 E-04$ & BIRC3,СCR7,CD40,CD83,CD86 & $155(16)$ \\
\hline crocidolite asbestos & chemical toxicant & & 1,985 & bias & $4,07 E-04$ & COI2,CD44,FOSL1,JUN,ZYX & $225(19)$ \\
\hline proteasome inhibitior PSI & chemical - protease inhibitor & & 1,342 & bias & $4,07 E-04$ & BMP2,CC12,FAS,MAPKG,RUN $\times 2$ & $192(16)$ \\
\hline Tgf beta & group & & 1,334 & bias & $4,09 E-04$ & 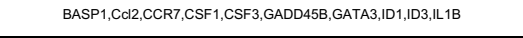 & $216(20)$ \\
\hline EIF2AK3 & kinase & & 1,846 & bias & $4,17 E-04$ & ANG,BIRC3,CCI2,FOSL1, IL 18,JUN,KLF4,RELB,RNASEL & $182(18)$ \\
\hline VIP & other & & $-0,009$ & & $4,17 E-04$ & 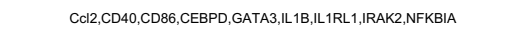 & $153(17)$ \\
\hline aspirin & chemical drug & Inhibitied & $-2,372$ & bias & $4,17 E-04$ & 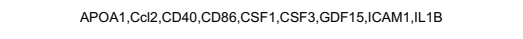 & ${ }^{122(16)}$ \\
\hline 1,2-dittiol-3-thione & chemical reagent & Activated & 3,560 & bias & $4,24 E-04$ & AKR1810,ALAS1,ANG,B8GGALN1,_ESD,FTH1,GSTA3,GSTP1,MGLL,NQO 1 & $45(3)$ \\
\hline vitamin E & chemical drug & & $-1,941$ & bias & $4,26 E-04$ & APOA1, CD44,Hamp/Hamp2,IL18,KLFF, SERP INE1,SLC11A2 2 & $177(19)$ \\
\hline LEF1 & transcription regulator & & 0,371 & bias & $4,26 E-04$ & 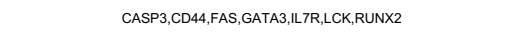 & $160(12)$ \\
\hline MBD2 & transcription regulator & & 0,115 & & $4,26 \mathrm{E}-04$ & AMY2A,BIRC3,GSTP1,HK2,OAT,PLA2G16, RELB & \\
\hline LI7RC & transmembrane receptor & & & & 4,29E-04 & PLET1,STEAP4 & \\
\hline ZNF385B & other & & & & $4,29 E-04$ & FAS,PERP & \\
\hline PARM1 & other & & & & $4,29 E-04$ & BMP2,BMP4 & \\
\hline diaziquone & chemical drug & & & & $4,29 E-04$ & CD40,IL1B & \\
\hline cobimetinib & chemical drug & & & & $4,29 E-04$ & $\mathrm{CD} 44, \mathrm{CSF} 3$ & \\
\hline cyclic AMP & chemical-endogenous mammalian & Activated & 2,925 & bias & $4,37 E-04$ & 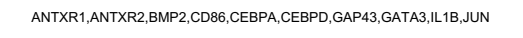 & $230(22)$ \\
\hline mannitol & chemical-endogenous mammalian & & 1,231 & & 4,43E-04 & CC12,1CAM1,JUN,SOD2 2 & ${ }_{174(21)}$ \\
\hline NR113 & ligand-dependent nuclear receptor & Activated & 2,759 & bias & $4,46 E-04$ & ALAS1,APOA1,CYP3A7,GADD45B, GSTP1, HK1,1СAM1,PGD,,STEAP4 & 139 (10) \\
\hline PPRC1 & transcription regulator & Activated & 2,449 & bias & $4,50 \mathrm{E}-04$ & ВMPG,GDF15,HK2,LAMB3,NFKBIZ,RND3 & \\
\hline GATA1 & transcription regulator & & 1,117 & bias & $4,57 E-04$ & 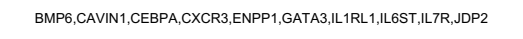 & \\
\hline EPO & cytokine & & $-0,404$ & bias & $4,57 E-04$ & 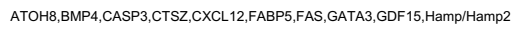 & $210(16)$ \\
\hline 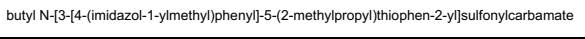 & chemical reagent & & & & $4,58 E-04$ & САSP3,|L1B,MLKL & \\
\hline CDSL & transmembrane recepptor & & & & $4,58 E-04$ & $\mathrm{COl2}, \mathrm{CClB}, \mathrm{LL1B}$ & $83(7)$ \\
\hline FEMMAA & transcription regulator & & & & $4,58 E-04$ & $\mathrm{COC2,CD68,LL1 \textrm {B }}$ & $143(13)$ \\
\hline GIPR & G-protein coupled receptor & & & & $4,58 E-04$ & $\mathrm{CO} 2, \mathrm{COClB}$, TCl7 & \\
\hline MAA-602 & chemical reagent & & & & $4,58 E-04$ & CASP3,CCL1,1118 & \\
\hline GSKJ4 & chemical reagent & & & & $4,58 \mathrm{E}-04$ & $\mathrm{CD40,CD83,CD86}$ & \\
\hline Fo gamma receptor & group & Activated & 2,200 & bias & $4,75 E-04$ & CCNA2, $, \mathrm{CD} 40, \mathrm{CD} 86$, ICAM1,TNFAIP3 & $177(17)$ \\
\hline TRB & transmembrane receptor & & 0,468 & bias & $4,75 E-04$ & CD44,CDBA,CXCR3,FAS,GATA3 & $147(11)$ \\
\hline 8.bromo-cAMP & chemical reagent & & $-0,731$ & bias & $4,79 E-04$ & 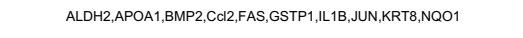 & $164(19)$ \\
\hline ATN1 & transcripition regulator & & & & $4,91 E-04$ & BASP1,BTG3, CAMK4, Cd24a, FF116,JUP, LTBP2, Marcks, TIAM1,TM4SF1 & \\
\hline glucocorticoid & chemical drug & & 0,268 & & $4,92 E-04$ & 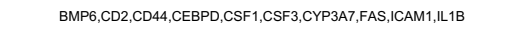 & $170(15)$ \\
\hline PTPNG & phosphatase & Inhibitied & $-2,412$ & bias & $5,04 E-04$ & CC12,CCR7, СD44, СD86, IL1B,LYYN & $155(16)$ \\
\hline zymosan & hemical - endogenous non-mammalia & & 1,988 & bias & $5,12 E-04$ & 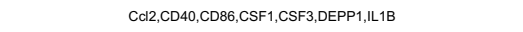 & 134 (15) \\
\hline
\end{tabular}




\begin{tabular}{|c|c|c|c|c|c|c|c|c|}
\hline 3,3-dindolylmethane & & chemical drug & & 1,937 & & $5,12 E-04$ & FAS,GDF15,HLA-A,IL1B,KRT19,NFKBAA,NQ0 1 & $215(21)$ \\
\hline PISK (family) & & group & & 1,510 & bias & 5,45E-04 & AKR1B10,BIRC3, CXCL12,FTH1,HK1, IL 18,NNQ01,PREX1, SERPINE1 & $195(20)$ \\
\hline ॥36A & & cytokine & Activated & 2,200 & bias & $5,50 \mathrm{E}-04$ & 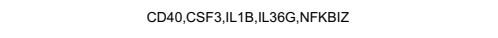 & $87(7)$ \\
\hline prostaglandin E1 & & chemical - endogenous mammalian & & $-1,192$ & bias & $5,51 E-04$ & CD14,CD40, CD86, САM1 & $211(19)$ \\
\hline TMSB4 & & group & & 1,964 & bias & $5,51 E-04$ & BMP2,BMP4,RUNX2,SERPINE1 & $132(12)$ \\
\hline ADRB3 & & G-protein coupled receptor & & & & $5,51 E-04$ & CCl2,CXCL12,IL18,JUN & 178 (19) \\
\hline BRC2 & & enzyme & & & & $5,51 E-04$ & BIRC3,CCNA2,CSF1,CSF3 & $146(11)$ \\
\hline Usp17la (includes others) & & peptidase & & 1,987 & bias & $5,51 E-04$ & COL2,CD44,L11, KLF 4 & $87(7)$ \\
\hline ethoxyquin & & chemical toxicant & & 1,934 & bias & $5,51 E-04$ & FTH1,GSTA3,GSTP1,NQO1 & \\
\hline metyrapone & & chemical drug & & 0,000 & bias & $5,51 E-04$ & ALAS1,CXCL12,ICAM1, IL1B & $124(11)$ \\
\hline СD38 & & enzyme & & 1,964 & bias & 5,53E-04 & 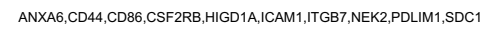 & \\
\hline Raf & & group & & & & $5,59 \mathrm{E}-04$ & FOSL1,GDF 15,|F116,JUN,RND3,SLC43AA, TNFAPP3 & $220(20)$ \\
\hline FANCC & & other & & & & $5,59 \mathrm{E}-04$ & CXCL12,GDF15,|L18,JAG1, OAT,RND3,TAX1BP3 & $147(16)$ \\
\hline твх21 & & transcription regulator & & $-0,180$ & & $5,59 E-04$ & CXCR3,GATA3,GZMA,LLRLL, ILGST,LLRR,TCF7 & 159 (15) \\
\hline ADAM10 & & peptidase & & & & $5,63 \mathrm{E}-04$ & CSF1,CXCL12,CXCL16,DTNA,L1366,IL6ST,LCK,SYT1 & \\
\hline NLRX1 & & other & Inhibited & $-2,449$ & bias & $5,63 E-04$ & 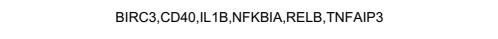 & $158(13)$ \\
\hline sphingosine-1-1phosphate & & chemical-endogenous mammalian & Activated & 2,044 & bias & $6,06 E-04$ & COL1, CD44,FAS, GAPA3,ICAM1,JUN,RUNX2,SERPINE1 & 167 (19) \\
\hline PDX1 & & transcription regulator & & 1,066 & bias & $6,08 E-04$ & 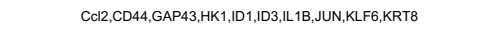 & $120(7)$ \\
\hline RIPK2 & 0,574 & kinase & Activated & 2,621 & bias & $6,11 E-04$ & ACOD1,CC12,CD40,CD86, ICAM1,RASGRP1,SOD2 & 132 (17) \\
\hline TERT & & enzyme & & $-0,686$ & & $6,19 E-04$ & 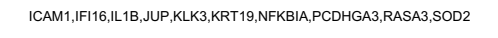 & $191(14)$ \\
\hline TSC2203 & & transcription regulator & & $-1,053$ & bias & $6,27 E-04$ & CD83,СD86, СEBPA,ICAM1,L118,RUNх2 & 161 (19) \\
\hline TIMP3 & & other & & $-0,816$ & bias & $6,27 E-04$ & BMP4,FAS,JUN,KLF4,LAMC2,SERPINE1 & $225(18)$ \\
\hline APOA 1 & 4,105 & transporter & & $-1,636$ & bias & $6,27 E-04$ & APOA 1, CCI2, ICAM1, II 18, SOD2,XDH & 153 (19) \\
\hline OSCAR & & other & & 1,342 & bias & 6,34E-04 & CD40,CD83, СD86, ICAM1,RRAS & $153(15)$ \\
\hline GATA3 & 7,490 & transcription regulator & & 0,470 & & 6,34E-04 & 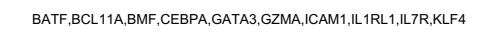 & $180(16)$ \\
\hline UCP1 & & transporter & & $-0,194$ & & $6,45 E-04$ & ALAS1,CASP3,,CD24a,CD68, GDF 15,GSTO1,L118,PGD,RNASEL,SERPINE1 & $85(7)$ \\
\hline CD36 & & transmembrane receptor & & 1,970 & bias & $6,52 E-04$ & APOA 1, CD86,, CSF 3, FAS, ICAM1, L1 18,LRP1,SERPINE1 & $162(18)$ \\
\hline AG490 & & chemical -kinase inhibitor & Inhibited & $-2,792$ & bias & $6,52 E-04$ & 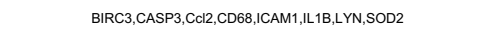 & $224(21)$ \\
\hline CCND1 & & transcription regulator & & 0,132 & & $6,57 \mathrm{E}-04$ & CCNA2,CD44,CD6,CDCA7,DONSON,HOMER2,HSPB 1, ID 1, ID3, KLKK3 & $137(7)$ \\
\hline melatonin & & chemical-endogenous mammalian & & 1,768 & & $6,60 E-04$ & BMP2,CASP3,FAS,GJB2,|L18,KRT 19,NQO1,RUNX2,SOD2 & 181 (19) \\
\hline nicotine & & chemical drug & & 1,115 & bias & $6,67 E-04$ & 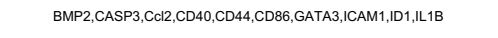 & $148(16)$ \\
\hline sphingosylphosphocholine & & chemical-endogenous mammalian & & & & $6,77 E-04$ & Col2,ICAM1,JUN & $185(19)$ \\
\hline c8 & & complex & & & & $6,77 E-04$ & COL2, ICAMM, LL1B & $107(9)$ \\
\hline SLC16A3 & & transporter & & & & $6,77 E-04$ & CASP3,HK2,LL1B & \\
\hline salmonella typhimurium lipopolysaccharide & & hemical - endogenous non-mammalia & & & & $6,77 E-04$ & $\mathrm{CO} 2, \mathrm{CD} 86, \mathrm{LL1B}$ & \\
\hline TNFRSF17 & & transmembrane receptor & & & & $6,77 E-04$ & CD40,CD86, ICAM1 & 136 (15) \\
\hline ATG16L1 & & enzyme & & & & $6,77 E-04$ & IL1B,NFKBIA,SOD2 & $112(9)$ \\
\hline
\end{tabular}




\begin{tabular}{|c|c|c|c|c|c|c|c|c|}
\hline RTKN & 1,276 & other & & & & $6,77 \mathrm{E}-04$ & BCLLA1,BIRC3,TNFAPB 3 & 91(9) \\
\hline $\mathrm{cr}_{7}$ & & other & & & & $6,77 E-04$ & COL2,ICAM1,L1B $1 \mathrm{~B}$ & $107(9)$ \\
\hline DEK & & transcription regulator & & & & $6,77 E-04$ & BIRC3,CCLI,NFKBIA & $95(8)$ \\
\hline 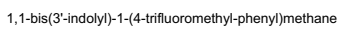 & & chemical reagent & & & & $6,77 \mathrm{E}-04$ & CAV2,GDF15,KLK3 & 160 (7) \\
\hline puromycin & & |hemical-endogenous non-mammalia & & & & $6,78 E-04$ & CASP3,CD40,LL1B,SERPINE1 & $186(22)$ \\
\hline TP53COR1 & & other & Activated & 2,000 & bias & $6,78 E-04$ & BIRC3,FAS,LL1, SOD2 & $174(13$ \\
\hline S-(2,3-bispalmitoyloxypropyl)-cystetene-GDPKHPKSF & & chemical reagent & & 1,974 & bias & $6,78 E-04$ & Apol7e (includes others), CD40, CD86, ICAM1 & $136(15$ \\
\hline Pkc(s) & & group & Activated & 2,673 & bias & 6,89E-04 & APOA1,DMD,FAS,FOSL1, GADD 45B, GAP43,IL18,JUN, KLF6,KRT8 & $207(19$ \\
\hline PRKAG3 & & other & & & & $7,14 \mathrm{E}-04$ & ALAS1,DDIT4L,FTL,ID1,KLF4,PLIN3,POLE4, RHOU,SLCZA3,TSPAN8 & \\
\hline GNA14 & & enzyme & & & & $7,24 E-04$ & 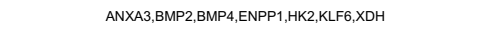 & \\
\hline andrographolide & & chemical drug & & 0,447 & & $7,27 E-04$ & CASP3,GATA3,GSTP1,ICAM1,L1L 1B & 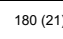 \\
\hline SN-38 & & chemical drug & & 1,982 & bias & $7,27 E-04$ & BIRC3,BMPG,FAS,GDF 15, HK2 & $183(14$ \\
\hline DDIT3 & & transcription regulator & & $-1,054$ & & 7,48E-04 & 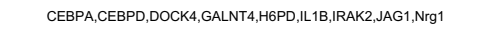 & $210(16)$ \\
\hline miR-146a-5p (and other mirNAs w/seed GAGAACU) & & mature microRNA & Inhibited & $-2,791$ & bias & 7,52E-04 & 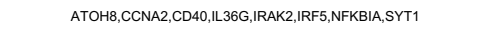 & $91(6)$ \\
\hline Rock & & group & & 1,664 & bias & $7,73 E-04$ & BMP2,CAVIN1,DMD, ICAM1,Nes, RUNX2 & $217(22$ \\
\hline NOG & & growth factor & & $-1,634$ & bias & 7,73E-04 & BMP2,BMPA,CD2,FAS, ID, RUN $\times 2$ & $189(23)$ \\
\hline PTPRJ & & phosphatase & Activated & 2,236 & bias & $7,73 E-04$ & CCNAZ,CEBPD,NFKBZZ,OASL,Vnn3,ZC3H12A & \\
\hline minocycline & & chemical drug & & $-1,775$ & bias & $7,87 E-04$ & СASP3,CC12, CD86, GATA3, ICAM1,LL18, IL6ST & $174(17$ \\
\hline YY1 & & transcription regulator & & 0,447 & & $8,01 E-04$ & 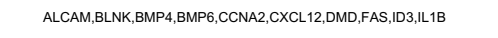 & $202(19$ \\
\hline HDAC3 & & transcription regulator & & $-0,751$ & bias & $8,07 E-04$ & BIRC3,BMP2, ICAM1,JUP,KLK3,NFKBIA,RELB,SERPINE1 & $218(23)$ \\
\hline Pka & & complex & & 1,915 & bias & $8,16 \mathrm{E}-04$ & ANTXR1,ANTXR2,CAMK4,CCR7,,CD14,CD40,CD44,JUN,KLFF,MEST & $200(18$ \\
\hline Fibrinogen & & complex & & 1,929 & bias & $8,24 E-04$ & CC12,CD40,ICAM1,LL1B & $133(17)$ \\
\hline ITGA9 & 0,739 & other & Activated & 2,000 & bias & $8,24 E-04$ & CCl12,CXCL12,CXCL14,IL1B & $146(12$ \\
\hline RPSGKA5 & & kinase & & & & $8,24 E-04$ & CCR7,FOSL1,L118,JUN & $191(21)$ \\
\hline PCDH11Y & & other & Activated & 2,000 & bias & $8,24 E-04$ & BMP4,CDA4,FOSL1,JUN & 130 (7) \\
\hline «37 & & cytokine & & $-1,938$ & bias & $8,24 E-04$ & ВMР2,СSF1,СSF3,L1L1B & $148(15$ \\
\hline CRNDE & & other & Activated & 2,236 & bias & $8,30 E-04$ & GAB1,GADD45B,RASA3,RRAS,TIAM1 & \\
\hline CASP1 & & peptidase & & 0,540 & bias & $8,30 \mathrm{E}-04$ & CCl2,CSF3,L1B,MLKL,RIPK2 & $180(177$ \\
\hline NCSTN & & peptidase & Inhibited & $-2,219$ & bias & $8,30 \mathrm{E}-04$ & CD14,CD68,CEBPA,CEBPD,CSF2RB & $66(7)$ \\
\hline SAA1 & & transporter & & 1,264 & bias & $8,30 E-04$ & COI2,CEBPA,CSF3,LL18,MRC1 & $194(16)$ \\
\hline MMP2 & & peptidase & Activated & 2,213 & bias & $8,30 \mathrm{E}-04$ & BMP2,CD44,CSF2RB,CXCL12,L66ST & $181(20)$ \\
\hline NLRP12 & & other & & $-1,206$ & bias & $8,30 \mathrm{E}-04$ & CXCL12,HLA-A,ICAM1,LL18,JUN & $144(16)$ \\
\hline FOSB & & transcription regulator & & & & $8,30 \mathrm{E}-04$ & CEBPA,FOSL1,FTH1,RUNX2,SERPINE1 & $181(15$ \\
\hline 1,4-4isis[2-(3,5-dichloropyridyloxy)]benzene & & chemical toxicant & & 1,548 & bias & $8,45 E-04$ & 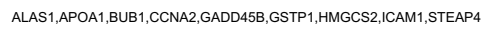 & $103(7)$ \\
\hline RNF31 & & enzyme & & $-1,890$ & bias & $8,54 \mathrm{E}-04$ & APOA1,CCI2,CCL18,CXCL16,IL1B, IL1RL1,1L366 & $162(12)$ \\
\hline lovastatin & & chemical drug & & 0.943 & bias & $8,61 E-04$ & CCNA2,CD866,GAP43,GATA3,IL18,KLF4,MAL,RHOU,SDC4,SERPINE1 & $191(20)$ \\
\hline ADIPOQ & & other & & 0,039 & & $8,69 E-04$ & 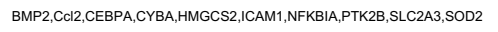 & $193(15$ \\
\hline
\end{tabular}




\begin{tabular}{|c|c|c|c|c|c|c|c|c|}
\hline PRL & & cytokine & Activated & 3,832 & bias & 8,92E-04 & ANXA3,CD40,CEBPD, GIycam1, ID 1, ID3,JUN,KRT 19, Marcks, MLKL & $208(20)$ \\
\hline IRF7 & & transcription regulator & Activated & 3,274 & bias & $9,12 E-04$ & 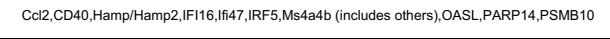 & $147(17)$ \\
\hline norepinephrine & & chemical-endogenous mammalian & & 1,363 & bias & $9,12 E-04$ & 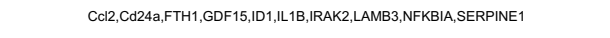 & $192(14)$ \\
\hline imiquimod & & chemical drug & Activated & 2,395 & bias & $9,25 E-04$ & CO12,CCR7,CD40, FOSL1,1CAM1,1L1B, OASL,SOD2 & $143(17)$ \\
\hline RELB & 0,509 & transcription regulator & & 0,807 & bias & $9,25 E-04$ & BIRC3,CD40,CXCL 12,LL18,NFKBIA,RELB,SOD2 & $103(11)$ \\
\hline levodopa & & chemical-endogenous mammalian & & $-0,470$ & & $9,39 E-04$ & AMIGO2,CASP3,Clec2d (includes others),CTSZ,CXCL12,DDIT4L,F11R,FOSL1,FTL,GADD45B & \\
\hline ТМЕМ173 & 1,650 & other & Activated & 2,414 & bias & $9,42 E-04$ & CD68, IF16,IL18, OASL,Serpina3g (includes others),UPP1 & $152(17)$ \\
\hline FGF10 & & growth factor & Activated & 2,425 & bias & $9,42 E-04$ & ANXA3,BMP4,PERP,SSa33,TMEM123,TSPAN8 & \\
\hline $\mathrm{Col} 2$ & 1,035 & cytokine & Activated & 2,190 & bias & $9,42 E-04$ & 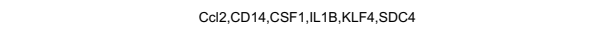 & $146(13)$ \\
\hline NPM1 & & transcription regulator & & & & $9,44 E-04$ & COI2,CEBPA,CSF1, 1L6ST,SOD2 & $203(16)$ \\
\hline xanthohumol & & chemical drug & & $-0,218$ & bias & $9,44 E-04$ & BIRC3,|СAM1,LL18,NQO1,RUN $\times 2$ & $142(10)$ \\
\hline $\mathrm{XDH}$ & 0,689 & enzyme & & $-0,094$ & & $9,44 E-04$ & CCI2, CEBPA,ID3, IL1B,SERPINE1 & $224(24)$ \\
\hline CD80 & & transmembrane receptor & & & & $9,44 E-04$ & CASP3, CD28,FAS, ICAM1,LL1B & $161(20)$ \\
\hline Irp & & group & & & & $9,52 E-04$ & FTH1,FTL,SLC11A2 2 & \\
\hline RBPJL & & transcription regulator & & & & $9,52 E-04$ & AMYZA,CPA2,CTRB2 & \\
\hline SELE & & transmembrane receptor & & & & 9,52E-04 & CCl1, CSF3,L11B & 148 (17) \\
\hline RANBP9 & & other & & & & $9,52 E-04$ & GAP43,JUN,PTK2B & $130(7)$ \\
\hline FTH1 & 0,558 & enzyme & & & & 9,52E-04 & CD86,FTH1,FTL & $36(3)$ \\
\hline TFR2 & & transporter & & & & $9,52 E-04$ & BMP6,GDF15,Hamp/Hamp2 & $120(9)$ \\
\hline sodium dodecyl sulfate & & chemical drug & & & & $9,52 E-04$ & FABP5, LL1B,SERPINE2 2 & \\
\hline clomipramine & & chemical drug & & & & $9,52 E-04$ & DEPP1,HPN,SLC2A3 & \\
\hline tin mesoporphyrin & & chemical drug & & & & $9,52 E-04$ & COL2,ICAM1,L1B & $99(7)$ \\
\hline pepstatin & & chemical - protease inhibitor & & & & $9,52 E-04$ & BMP2,CD9,STC2 & \\
\hline perilla alcohol & & chemical drug & & & & $9,52 E-04$ & BCL2A1,LL1B,NFKBIA & $116(11)$ \\
\hline NRAS & & enzyme & Inhibited & $-2,804$ & bias & $9,56 E-04$ & 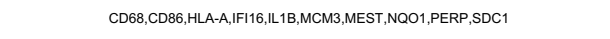 & 159 (17) \\
\hline ॥.27 & & cytokine & & 1,444 & bias & $9,57 \mathrm{E}-04$ & 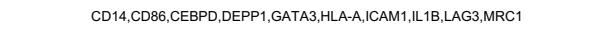 & $185(17)$ \\
\hline If gamma & & complex & Activated & 2,226 & bias & $9,90 \mathrm{E}-04$ & 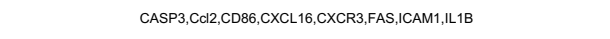 & $157(20)$ \\
\hline lactosylceramide & & chemical - endogenous mammalian & & & & $9,91 E-04$ & CO12,CD40,ICAM1, RLLB & $144(15)$ \\
\hline Pkg & & group & & 1,067 & bias & $9,91 E-04$ & HK2,JUN,SOD2,VCL & $180(16)$ \\
\hline EIFAG2 & & translation regulator & & 1,000 & & $9,91 E-04$ & JUN,KLF4,KRT19,SERPINE2 & \\
\hline LRPAP1 & & other & & $-0,308$ & bias & $9,91 E-04$ & CC12,IL18,LRP1,SERPINE1 & $144(16)$ \\
\hline EPCAM & & other & & $-0,762$ & & 9,91E-04 & FABP5,FOSL $1,1 \mathrm{DD}, \mathrm{JUN}$ & $164(13)$ \\
\hline GRN & & growth factor & & & & $9,91 E-04$ & CCl2,CTSZ,ICAM1,L11B & $212(19)$ \\
\hline ketamine & & chemical drug & & & & $9,91 E-04$ & CASPB,HK1,KLF, NQO1 & $102(7)$ \\
\hline apicidin & & hemical - endogenous non-mammalia & & 0,000 & & 9,91E-04 & AMPD3,FAS,HGPD,ITGB7 & \\
\hline IF16 & 2,317 & transcription regulator & & 1,815 & bias & $1,00 E-03$ & 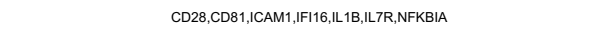 & $211(20)$ \\
\hline нтт & & transcription regulator & & 1,372 & & $1,00 \mathrm{E}-03$ & 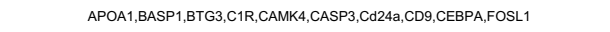 & 232 (21) \\
\hline
\end{tabular}




\begin{tabular}{|c|c|c|c|c|c|c|c|c|}
\hline PARP1 & & enzyme & & 0,537 & bias & 1,01E-03 & CO12,CXCL12,FAS,GBP6,GDF15,ICAM1,L118,JUN,KLK3 & $215(20)$ \\
\hline littium chloride & & chemical drug & & 0,923 & bias & $1,01 E-03$ & B4GALT1,BMP2,BMP4,CD44,GATA3,GDF 15,KLK3,ККRT20,Marcks & $219(26)$ \\
\hline CBL & & transcription regulator & & & & $1,04 E-03$ & CSF2RB,FLT3,LL1B,LCK,LYN,RUNX2 & $110(7)$ \\
\hline cholesterol & & chemical-endogenous mammalian & & 1,021 & bias & $1,04 E-03$ & CAMK1D,CAV2,CC12,CD14,CD68,,CSF1,FABP5,ICAM1,L118,SMO & 137 (17) \\
\hline кмт2D & & transcription regulator & & 1,372 & bias & $1,04 E-03$ & BTG3,CDCA7,GJB2,GPRC5C,KLF4, KRT23,_AMB3,LCK,POLE4,PPIC & \\
\hline TLR2 & & transmembrane receptor & Activated & 3,226 & bias & $1,05 E-03$ & BMP2,CC12,CD40,CD866,CEBPD,CSF3,,GATA3,ICAM1,LL18,IRAK3 & $148(18)$ \\
\hline Iysophosphatidicic acid & & chemical - other & Activated & 2,781 & bias & $1,06 E-03$ & CO12,CD14,CSF1, ICAM1,LL18,UUN,RALA,SERPINE 1 & 149 (19) \\
\hline NQO1 & 1,033 & enzyme & & $-1,387$ & & $1,07 E-03$ & BIRC3,CCI8,CEBPA,CXCL12,CXCR3 & $105(7)$ \\
\hline IGHM & & transmembrane receptor & & $-0,577$ & & $1,07 E-03$ & CAl2,CD86,FAS,LL1B,JUN & $191(20)$ \\
\hline mevalonic acid & & chemical-endogenous mammalian & & 1,477 & & $1,07 E-03$ & APOA1,CD 40, ICAM1,LL18,SERPINE1 & $171(19)$ \\
\hline CLDN7 & & other & & 0,990 & & $1,07 E-03$ & ANXA3,B4GALNT1,CD68,FADS3,FOSL1,PLA2 616, SERPINB1,SYTLL, TINAGL1 & \\
\hline Growth hormone & & group & & 1,098 & bias & $1,08 E-03$ & 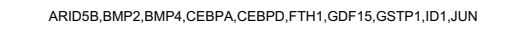 & $227(18)$ \\
\hline Notch & & group & & 1,144 & bias & $1,08 E-03$ & CDCA7,GADD45B, GATA3, ID, ID, JAG 1 , Nes & ${ }^{123(14)}$ \\
\hline Sos & & group & & & & $1,09 E-03$ & B4GAL T1,CD44,DOCK1,HPGDS,LL36G,KLFG,MAPKG,Nrg1,SEMA3B,SEMA3E & \\
\hline 2-(-4-amino-1-1-isopropyy-1H-pyrazoolo[3,4-d-djpyrimididin-3-y)|-1H-indol-5-ol & & chemical reagent & & 0,859 & & 1,10E-03 & BMP2,G1R,CDA4,FOSL1,1D1,1D3,KLFF,MCM3,SDC4,SERPINB1 & $118(12)$ \\
\hline estrogen & & chemical drug & Activated & 2,552 & bias & $1,10 \mathrm{E}-03$ & 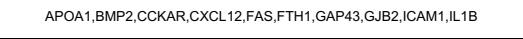 & $178(20)$ \\
\hline adenosine & & chemical-endogenous mammalian & & $-0,394$ & & 1,14E-03 & CD28, CD86, ICAM1,LL1 B, Saa3,SERPINE1 & $197(21)$ \\
\hline CLEC4G & & other & & 0,816 & bias & 1,14E-03 & 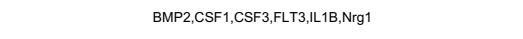 & \\
\hline PAX5 & & transcription regulator & & $-0,730$ & & 1,14E-03 & BLNK,CD40,FLT3,HK2,SDC1,SLC2A3 & \\
\hline FOSL2 & & transcription regulator & Activated & 2,219 & bias & 1,14E-03 & Col2,FAS,FOSL1,LL1B,RELB,RUNX2 & $130(11)$ \\
\hline niacinamide & & chemical-endogenous mammalian & & $-1,698$ & & 1,14E-03 & BCL2A1,CD14,CD40,CXCL12,ICAM1,SLC34A2 & $149(15)$ \\
\hline CTR9 & & other & & 1,000 & & $1,18 E-03$ & CXCL12,RASGRP1,SDC1,TIAM1 & \\
\hline epicatechin gallate & & chemical drug & & & & $1,18 \mathrm{E}-03$ & GDF15,ICAM1,LYN,PERP & $96(7)$ \\
\hline evodiamine & & hremical - endogenous non-mammalia & & $-1,715$ & bias & $1,18 E-03$ & BCL2A1,BRC3,CASP3,ICAM1 & $86(7)$ \\
\hline chelerythrine & & chemical drug & & $-1,000$ & bias & 1,18E-03 & CCI2,CYBA,ICAMM1,NFKBIA & $110(14)$ \\
\hline TNFRSF1B & & transmembrane recepptor & & 1,809 & bias & $1,20 E-03$ & CASP3, CXCL 12,FAS, ICAM1,JUN,SERPINE1,SOD2, TNFAP3 & $134(16)$ \\
\hline MEOX2 & & transcription regulator & & $-1,400$ & bias & $1,21 E-03$ & ICAM1,ID1,ID3,LRP1,TNFAIP3 & \\
\hline NR2F1 & & \begin{tabular}{|l|l|l|l} 
Iigand-dependent nuclear recepptor \\
\end{tabular} & & 0,000 & & $1,21 E-03$ & ALDH2,APOA1,FNBP1,GDF15,JAG1 & \\
\hline mitoxantrone & & chemical drug & & 1,067 & bias & $1,21 E-03$ & FAS, IF16,IL1B, KLKз,PERP & $167(13)$ \\
\hline paricalcitol & & chemical drug & & $-1,000$ & bias & $1,21 E-03$ & ВМР2,СС2,,СРРЗА, ,ІСАМ1, L1В & 155 (17) \\
\hline SIRT6 & & enzyme & & & & $1,25 E-03$ & BIRC3,CCI2,CSF1,LL1RL1, RAK3,SOD2 & $212(16)$ \\
\hline miR-145-5p (and other miRNAs w/seed UCCAGUU) & & mature microRNA & Inhibited & $-2,411$ & bias & $1,25 E-03$ & CCNA2,F11R,KLFA,RTKN,SERPINE1,SOD2 & \\
\hline CD14 & 1,316 & transmembrane receptor & Activated & 2,392 & bias & $1,25 E-03$ & BCL2A1,CCI2,CXCL12,ICAM1,L118,TNFAPP3 & $144(16)$ \\
\hline MAPRK $1 / 2$ & & group & & 1,772 & bias & $1,26 \mathrm{E}-03$ & COL2,FOSL1, HK2,HSPB 1,L118,JAG1,JUN & $189(21)$ \\
\hline PTPN11 & & phosphatase & & $-1,254$ & & $1,26 \mathrm{E}-03$ & CD14,CD68, CEBPA,CSF1,GAB1,JUN,PERP & ${ }_{194(23)}$ \\
\hline SFTPA1 & & transporter & & $-1,087$ & bias & $1,26 \mathrm{E}-03$ & CO12,GPR68,IL18,|LIRL1,MRC1,SERPINE1,TCEA3 & $138(15)$ \\
\hline galactosylceramide & & chemical- endogenous mammalian & & & & $1,27 \mathrm{E}-03$ & $\mathrm{CD} 40, \mathrm{CD} 86$ & \\
\hline
\end{tabular}




\begin{tabular}{|c|c|c|c|c|c|c|c|c|}
\hline C1QTNF12 & & other & & & & $1,27 E-03$ & $\mathrm{CO12,111 \textrm {B }}$ & $103(7)$ \\
\hline NIPBL & & transcription regulator & & & & $1,27 E-03$ & KLF4,POLE4 & \\
\hline ASH1L & & transcription regulator & & & & $1,27 E-03$ & IRAK3,TNFAIP3 & \\
\hline КLHL21 & & other & & & & $1,27 E-03$ & NFKBIA,NFKBIZ & \\
\hline CNDP2 & & peptidase & & & & $1,27 E-03$ & CD44,CXCR3 & \\
\hline Ap1 gamma & & group & & & & $1,27 E-03$ & CD28,HLA-A & \\
\hline CARDB & & other & & & & $1,27 E-03$ & BCL2A1,L1B & \\
\hline FтMT & & enzyme & & & & $1,27 E-03$ & FTH1,FTL & \\
\hline STRAP & & other & & & & $1,27 E-03$ & JUN,SERPINE1 & \\
\hline Defb2 & & other & & & & $1,27 E-03$ & $\mathrm{Cd} 24 \mathrm{a}, \mathrm{CD} 40$ & \\
\hline TIGAR & & enzyme & & & & $1,27 E-03$ & CCl2,TNFAPB 3 & $142(10)$ \\
\hline 1-heptanol & & chemical reagent & & & & $1,27 E-03$ & CD40,CD86 & \\
\hline ellipiticine & & chemical reagent & & & & $1,27 E-03$ & CD40,IL1B & \\
\hline prostratin & & hremical - endogenous non-mammalia & & & & $1,27 E-03$ & CASP3, ICAM1 & $95(7)$ \\
\hline Mn2+ & & chemical - endogenous mammalian & & & & $1,27 E-03$ & GAP43,ICAM1 & \\
\hline teriflunomide & & chemical drug & & & & $1,29 E-03$ & FAS, ICAM1,L1B & 126 (11) \\
\hline Traj18 & & other & & & & $1,29 E-03$ & $\mathrm{CCl} 2, \mathrm{CD} 40, \mathrm{CD} 86$ & 135 (11) \\
\hline GPR132 & & G-protetin coupled receptor & & & & $1,29 \mathrm{E}-03$ & CO12,ICAM1,MRC1 & 175 (13) \\
\hline ACVR2A & & kinase & & & & $1,29 E-03$ & HampHamp2,|CAM1, ID1 & $97(4)$ \\
\hline APOH & & transporter & & & & $1,29 E-03$ & $\mathrm{CD} 40, \mathrm{CD} 83, \mathrm{CD} 86$ & $156(18)$ \\
\hline MAS1 & & G-protein coupled receptor & & & & $1,29 E-03$ & CA12,L18B,MRC1 & 179 (17) \\
\hline NDFIP1 & & other & & & & $1,29 E-03$ & "L1B,LYN,SLC11A2 2 & \\
\hline 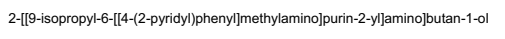 & & chemical - kinase inhibitor & & & & $1,29 E-03$ & $\mathrm{CCl} 2, \mathrm{CCNA} 2, \mathrm{CD} 86$ & \\
\hline Ifnz (includes others) & & cytokine & & & & $1,29 E-03$ & CD40,CD83,,CD86 & $119(8)$ \\
\hline нмох1 & & enzyme & & 0,000 & & $1,34 E-03$ & 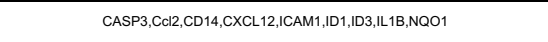 & $197(21)$ \\
\hline ERG & & transcription regulator & & 1,000 & bias & $1,34 E-03$ & 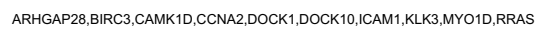 & \\
\hline TSLP & & cytokine & & 1,966 & bias & $1,35 E-03$ & CCR7, СD40,СD83,СD86,ICAM1 & $154(16)$ \\
\hline CEACAM1 & & transporter & & 1,342 & bias & $1,35 E-03$ & ANG,CD40,CD86, CSF3, ICAM1 & $176(18)$ \\
\hline Tetr & 4,054 & transcription regulator & & $-1,167$ & bias & $1,35 E-03$ & BATF,GATA3,GZMA,LLRR,LYN & \\
\hline monocrotaline & & chemical toxicant & & 0,600 & & $1,35 E-03$ & 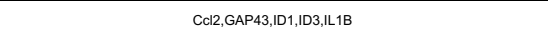 & $103(7)$ \\
\hline RETNLB & & other & Activated & 2,630 & bias & $1,36 E-03$ & 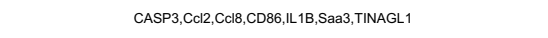 & $197(18)$ \\
\hline Gsk3 & & group & & 0,911 & bias & $1,36 E-03$ & CD40,CEBPA,GDF15,ICAM1,1L18,JUN,TNFAIP3 & $185(16)$ \\
\hline JUND & & transcription regulator & & & & $1,36 \mathrm{E}-03$ & ALAS1,CCI8,CXCL16,FOSL1,FTH1,RUNX2,SDC1 & $91(9)$ \\
\hline cigltiazone & & chemical drug & & $-0,509$ & & $1,36 E-03$ & CAV2,CC12,ICAM1,JUN,KLK3,NFKB|A|,SERPINE1 & $247(24)$ \\
\hline 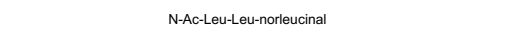 & & chemical - protease inhibitior & & $-0,506$ & bias & $1,36 E-03$ & 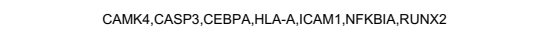 & $232(22)$ \\
\hline dehydroisoandrosterone & & chemical-endogenous mammalian & & $-0,742$ & & $1,36 \mathrm{E}-03$ & BMP4,CI21,ICAM1,KLK3,NFKBIA,RACK1,Saa3 & $197(22)$ \\
\hline แ12B & & cytokine & & 1,525 & bias & $1,37 \mathrm{E}-03$ & 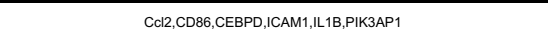 & $182(18)$ \\
\hline
\end{tabular}




\begin{tabular}{|c|c|c|c|c|c|c|c|c|}
\hline TCF12 & & transcription regulator & & 0,655 & bias & $1,37 E-03$ & 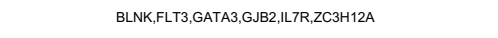 & $30(2)$ \\
\hline sodium arsenite & & chemical drug & & 1,400 & bias & $1,37 E-03$ & CEBPA,FTH1,GADD45B,HK2,JUN,LITAF & $187(18)$ \\
\hline TFAP2A & & transcription regulator & & $-1,623$ & & $1,37 E-03$ & ALCAM,BIRC3,CEBPA,KLF4,PLA2G16,RAB27B,SERPINE1,S0D2 & $195(16)$ \\
\hline INSIG1 & & other & Inhibitied & $-2,828$ & bias & $1,37 E-03$ & CCl1,CCR7,CD68, СLEC4D,FABP5, HMGCS2,MRC1,NFKBIZ & \\
\hline BRCA1 & & transcription regulator & Activated & 2,319 & bias & $1,38 E-03$ & 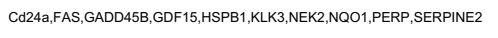 & $198(17)$ \\
\hline zerumbone & & hemical - endogenous non-mammalia & & $-0,832$ & bias & 1,39E-03 & BCL2A1,BIRC3,CASP3,ICAM1 & $101(10)$ \\
\hline NFAT (complex) & & complex & & & & $1,39 E-03$ & CD68, CD86, IICAM1,SERPINE 1 & \\
\hline TRPS1 & & transcription regulator & & 0,152 & & $1,39 E-03$ & ENPP1,GALNT3,RUNX2,SERPING1 & \\
\hline МАРЗКЗ & & kinase & & & & $1,39 E-03$ & BMP2,JUN,STEAP4,TNFAIP3 & 150 (17) \\
\hline CLDN6 & & other & & & & $1,39 E-03$ & CLDN10,CLDN4,KLF4,TGM3 & \\
\hline TRPV 4 & & ion channel & Activated & 2,000 & bias & 1,39E-03 & $\mathrm{CCl1}, \mathrm{Cl} 18, \mathrm{FAS}, \mathrm{Saa} 3$ & $113(7)$ \\
\hline SB 290157 & & chemical reagent & Inhibited & $-2,000$ & bias & 1,39E-03 & ВMP4,СD40,CD86,L1L1B & $176(13)$ \\
\hline GL1 & & transcription regulator & Activated & 2,527 & bias & $1,40 \mathrm{E}-03$ & 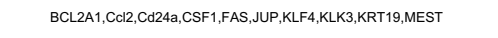 & $139(7)$ \\
\hline 4-hydroxytamoxifen & & chemical drug & Activated & 2,359 & bias & 1,44E-03 & CDCA7,CEBPA,CEBPD,CLDNA,LZR,JUN,KRT19,NFKBAA,RND1,TM4SF1 & $215(22)$ \\
\hline Z-LLL-CHO & & chemical - protease inhibitor & & 1,791 & bias & $1,46 \mathrm{E}-03$ & 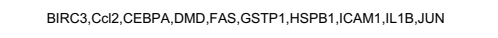 & $217(22)$ \\
\hline USF1 & & transcription regulator & & 1,925 & bias & $1,46 E-03$ & 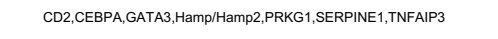 & \\
\hline NCOA3 & & transcription regulator & Activated & 2,592 & bias & 1,46E-03 & CCNA2,CEBPA,GJB2,HK2,MUC1,NFKBAA,XDH & 151 (11) \\
\hline KLFG & 1,036 & transcription regulator & & 1,303 & & 1,46E-03 & CC12,1L18,MRC1,NFKBAA,SDC1,TNFAIP3,TNP1 & $154(16)$ \\
\hline PRNP & & other & & 0,059 & & $1,46 \mathrm{E}-03$ & CASP3, CD44,FOSL1,HSPB 1,LL18,LRP 1,NFKBIA & $212(20)$ \\
\hline STAT2 & & transcription regulator & & 1,980 & bias & 1,49E-03 & $\mathrm{CC} 12, \mathrm{CD} 40, \mathrm{CDB8}, \mathrm{GBP}, \mathrm{If47} 7, \mathrm{IRF} 5$ & $137(10)$ \\
\hline GPS2 & & transcription regulator & & $-1,706$ & bias & 1,49E-03 & BLNK,CCL12,CD14,DHRS3,NFKBA,ZZNF703 & \\
\hline diphenyleneiodonium & & chemical reagent & Inhibited & $-2,400$ & bias & $1,49 E-03$ & BMP2,CC12,CLNN4,L11, SERPINE1,SOD2 & $189(18)$ \\
\hline DICER1 & & enzyme & Inhibited & $-2,621$ & & 1,49E-03 & 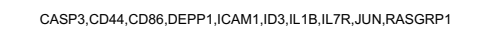 & $210(22)$ \\
\hline CDKN2A & & transcription regulator & & $-0,432$ & & 1,49E-03 & 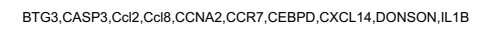 & $226(22)$ \\
\hline $\mathrm{FN} 1$ & & enzyme & & 1,571 & bias & $1,52 \mathrm{E}-03$ & 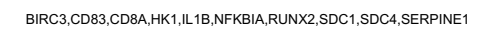 & $215(17)$ \\
\hline RNASEHZA & & enzyme & & & & $1,52 E-03$ & IF16,IFNGR2,IRF5,NFKBAA,OASL & \\
\hline TLR7/8 & & group & Activated & 2,000 & bias & $1,52 \mathrm{E}-03$ & BCLZA1,NFKBIA,NFKBIZ,PNRC1,TNFAPP3 & $107(7)$ \\
\hline TNFSF12 & & cytokine & Activated & 2,747 & bias & $1,55 E-03$ & BST1,CC12,CD68,CLEC4D, ICAM1,LL18,NFKBBA,TNFRSF $12 A$ & $174(17)$ \\
\hline PTGER2 & & G-protein coupled receptor & & 1,725 & bias & 1,55E-03 & CCNA2,CCR7, CLEC4D,ESD,LL18, IL366, NEK2,SERPINE1 & $157(17)$ \\
\hline TNFRSFB & & transmembrane receptor & & 0,816 & & 1,63E-03 & CCR7,FAS, FIIG,LCP2, RLLB, VCL & \\
\hline NODAL & & growth factor & & $-1,000$ & & 1,63E-03 & $\mathrm{CCR7,CD40,CD86,MRC1}$ & $94(7)$ \\
\hline SLPI & & other & & $-0,762$ & bias & 1,63E-03 & CD40,CD86, ICAM1,NFKBIA & 126 (16) \\
\hline NFKBBZ & 0,499 & transcription regulator & & 1,960 & bias & 1,63E-03 & CCl2,CEBPD,CSF3,LL36G & 189 (12) \\
\hline HSPD1 & & enzyme & & 1,969 & bias & $1,63 E-03$ & CO12,GATAB, ICAM, IL1B & $156(19)$ \\
\hline ochratoxin A & & chemical toxicant & & & & 1,63E-03 & CASP3,FAS,GSTP1,NQ01 & \\
\hline IRS1 & & enzyme & & 1,054 & bias & $1,66 \mathrm{E}-03$ & 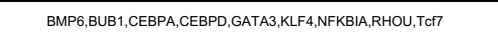 & $173(12)$ \\
\hline chloropromazine & & chemical drug & & $-0,555$ & bias & 1,69E-03 & CD83,DEPP1,HPN,|L18,SLCZA3 & \\
\hline
\end{tabular}




\begin{tabular}{|c|c|c|c|c|c|c|c|c|}
\hline azithromycin & & chemical drug & & & & $1,69 \mathrm{E}-03$ & Col2,LL18,MRC1 & $199(20)$ \\
\hline iclilin & & chemical reagent & & & & $1,69 \mathrm{E}-03$ & CCl2,CSF3,LL1B & \\
\hline chloryclizine & & chemical drug & & & & $1,69 \mathrm{E}-03$ & DEPP1,HPN,SLCZA3 & \\
\hline wwc1 & 1,521 & transcription regulator & & & & $1,69 \mathrm{E}-03$ & BMP2,RASSF4,YAP1 & $49(6)$ \\
\hline LECT2 & & other & & & & $1,69 \mathrm{E}-03$ & $\mathrm{CAl} 2, \mathrm{CSF} 3, \mathrm{LL} 1 \mathrm{~B}$ & $123(9)$ \\
\hline АнзквР1 & & other & & & & $1,69 \mathrm{E}-03$ & BCL2A1,CDB6,SERPINE 1 & $86(6)$ \\
\hline PTGIR & & G-protein coupled receptor & & & & $1,69 \mathrm{E}-03$ & COL12,CXCL12,L11B & $169(16)$ \\
\hline c9 & & other & & & & $1,69 \mathrm{E}-03$ & COL2,ICAM1,L1B & $107(9)$ \\
\hline CACNA1A & & ion channel & & & & $1,69 \mathrm{E}-03$ & $\mathrm{CCl} 2, \mathrm{CD} 68, \mathrm{LL} 1 \mathrm{~B}$ & \\
\hline CXCL1 & & cytokine & & & & $1,69 \mathrm{E}-03$ & CCI2,ICAM1,L1B & $135(12)$ \\
\hline LY-2510924 & & biologic drug & & & & $1,69 \mathrm{E}-03$ & BUB1,CD14,LLRB3 & \\
\hline edaravone & & chemical drug & & & & $1,69 \mathrm{E}-03$ & CASP3,CCl2,FAS & $180(20)$ \\
\hline SR 144528 & & chemical reagent & & & & $1,69 \mathrm{E}-03$ & ICAM1,LLBB,NFKB|A & $187(16)$ \\
\hline 5-fluorouracii & & chemical drug & Activated & 2,031 & & $1,70 \mathrm{E}-03$ & CASP $3, \mathrm{CD} 40, \mathrm{CD} 81, \mathrm{CD} 86, \mathrm{FAS}, \mathrm{GAP} 43, \mathrm{GDF} 15, \mathrm{Ll} 1 \mathrm{11}, \mathrm{JUN}, \mathrm{RHBDF} 2$ & $207(19)$ \\
\hline 5-N-ethylcarboxamido adenosine & & chemical reagent & & $-0,707$ & & $1,75 E-03$ & CC12,CEBPD,CSF2RB,DUSP16,HK2,LL18,KLFG,SERPINE1 & $177(15)$ \\
\hline $\mathrm{TSC2}$ & & other & Inhibitied & $-2,355$ & bias & 1,75E-03 & CC12,CD68, CP, GAP43,HK2, HMGCS2,HSPB1,RASSF4,SOD2 & $198(19)$ \\
\hline EGLN1 & & enzyme & & 1,000 & & $1,77 \mathrm{E}-03$ & CO12,CD86, HamplHamp2,ICAM1,L118,SDC4 & 1966 (19) \\
\hline RHO & & G-protein coupled receptor & & $-0,447$ & & $1,77 E-03$ & CASP3,CD44,CEBPD,HK2,SERPING1,TNFAIP3 & \\
\hline SIN3A & & transcription regulator & & & & $1,77 E-03$ & CASP3,GADD45B,GSTP1,KLFF,KLK3,SERPINE1 & $162(11)$ \\
\hline IRF1 & & transcription regulator & Activated & 3,056 & bias & $1,79 E-03$ & 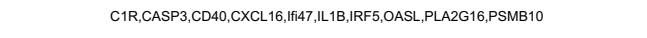 & $168(16)$ \\
\hline $\mathrm{CDH} 1$ & & other & & 0,051 & & $1,80 E-03$ & BMP4,CD44,FOSL1,JUN,JUP, KRT19,RALA & $213(22)$ \\
\hline CFTR & & ion channel & & $-0,896$ & & $1,80 E-03$ & BLNK,CFI,FAS,NFKBIA,NFKBIZ,SSa3,,TNFAIP3 & $133(14)$ \\
\hline phytohemagglutinin & & chemical drug & Activated & 2,750 & bias & $1,81 \mathrm{E}-03$ & ALDH2,CASP3,CCR7,,CD44,CD83,CSF1,CXCL12,FAS, GATA3,ICAM1 & 173 (19) \\
\hline CD24 & & other & & & & $1,85 E-03$ & 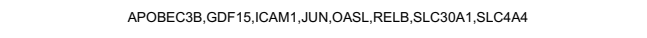 & $135(7)$ \\
\hline ACKR2 & & G-protein coupled receptor & Inhibited & $-2,236$ & bias & $1,88 E-03$ & ACOD1,F116, Ifi47, ILIB, OASL & \\
\hline D-galactosamine & & chemical - endogenous mammalian & Activated & 2,219 & bias & $1,88 E-03$ & CD14,GDF15,ICAM1, JUN,SOD2 & 148 (17) \\
\hline TAF4B & & transcription regulator & & & & $1,90 \mathrm{E}-03$ & JUN,NFKBA,TNFAPР,ZBTB16 & \\
\hline OSMR & & transmembrane receptor & & 1,969 & bias & $1,92 E-03$ & BIRC3, CCI2,GAP43,Ifi7, 7L6ST,Serpina3g (includes others) & 175 (18) \\
\hline usF2 2 & & transcription regulator & & & & $1,92 E-03$ & CD2,GATA3,Hamp/Hamp2,KLKK,PRKG1,SERPINE1 & \\
\hline HDAC4 & & transcription regulator & & & & $2,04 E-03$ & DMD,FOSL1,HOMER2,JUN,RAB3C,RTN1,RUNX2,SYN2,TNFRSF12A & \\
\hline LCN2 & & transporter & & 0,163 & bias & $2,06 E-03$ & BMP2,CC12,Cl18,L118,MRC1,RUNX2,TINAGL1 & $116(9)$ \\
\hline amphetamine & & chemical drug & & 1,050 & bias & $2,06 E-03$ & BMP4,BUB1,FOSL 1,GRM8, ICAM1,JUN,TNFAIP3 & $106(7)$ \\
\hline ATP & & chemical - endogenous mammalian & Activated & 2,515 & bias & $2,08 E-03$ & CO12,CCR7,CD14,CDB3, CD86, ICAM1, L1 18,SERPINE 1 & $150(18)$ \\
\hline EGLN & & group & Inhibitied & $-3,109$ & bias & $2,09 E-03$ & CAVII1,CCL2,FTL,GATA3,HamplHamp2,HK2,RNASE4,Serpina3g (includes others),SERPINE1,STC2 & $151(7)$ \\
\hline ressatorid & & chemical drug & & & & $2,17 E-03$ & АРОВЕСЗВ,СL1, 1113 & $134(16)$ \\
\hline perhexiline & & chemical drug & & & & $2,17 E-03$ & DEPP1,HPN,SLCZA3 & \\
\hline LLRA2 & & other & & & & $2,17 E-03$ & $\mathrm{CD} 40, \mathrm{CSF} 3, \mathrm{MRC} 1$ & \\
\hline
\end{tabular}




\begin{tabular}{|c|c|c|c|c|c|c|c|c|}
\hline ERFE & & other & & & & $2,17 E-03$ & Атон8,Наamp/Hamp2,101 & \\
\hline mir-373 & & microRNA & & & & $2,17 E-03$ & CD44,LATS2,SERPINE1 & \\
\hline FSTL1 & & other & & & & $2,17 E-03$ & COI2,GDF15,IL1B & $146(16)$ \\
\hline ATXN3 & & peptidase & & & & $2,17 E-03$ & CXCL12,LLRL1,SOD2 & \\
\hline KRT8 & 0,955 & other & & & & $2,17 \mathrm{E}-03$ & KRT19,KRT20,Reg3d & \\
\hline CCL3L3 & & cytokine & & & & $2,17 E-03$ & $\mathrm{CCl2,CD40,IL1B}$ & $135(13)$ \\
\hline SIRPA & & phosphatase & & & & $2,17 E-03$ & CCl2,CSF3,LL1B & $150(17)$ \\
\hline hydroquinone & & chemical- endogenous mammalian & & & & $2,17 E-03$ & แLB, JUN,SFN & $148(12)$ \\
\hline MRTFA & & transcription regulator & & 0,946 & & $2,19 \mathrm{E}-03$ & 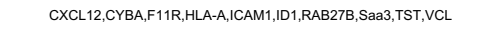 & $29(3)$ \\
\hline UTP & & chemical-endogenous mammalian & & & & $2,19 E-03$ & COI2,CD14,ICAM1,SERPINE1 & $161(18)$ \\
\hline PROCR & & other & & $-0,747$ & bias & $2,19 E-03$ & CC12,GBP, ,ICAM1,L11B & 169 (19) \\
\hline BCAP31 & & transporter & & $-0,152$ & bias & $2,19 \mathrm{E}-03$ & CO12,CD81, CD9, L118 & \\
\hline BGN & & other & & 0,882 & bias & $2,19 \mathrm{E}-03$ & BMP2,L18,RUNX2,SERPINE1 & $220(22)$ \\
\hline Sаa 3 & 1,778 & other & & & & $2,19 \mathrm{E}-03$ & APOA $1, B M P 2$, CSF 3, RUN $\times 2$ & \\
\hline SLC9A3 & & ion channel & & & & $2,19 E-03$ & Ifi47,Reg3d,SSa3,_UPP1 & \\
\hline BMPR1B & & kinase & & 1,969 & bias & $2,19 \mathrm{E}-03$ & ID1,1D3,RUNX2,SERPINE1 & $176(17)$ \\
\hline dially disulfide & & hemical - endogenous non-mammaila & & 1,980 & bias & $2,19 E-03$ & CASP3,GDF15,GSTA3,NQO 1 & \\
\hline ceramide & & chemical-endogenous mammalian & & 1,091 & bias & $2,19 \mathrm{E}-03$ & САSP, & $169(21)$ \\
\hline NCOA2 & & transcription regulator & & 0,964 & bias & $2,20 \mathrm{E}-03$ & 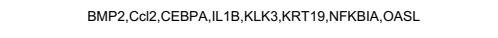 & $196(18)$ \\
\hline arsentite & & chemical toxicant & & & & $2,20 \mathrm{E}-03$ & ESD,GSTP1,Hspa1b,HSPB1, JUN,NQO1,PRKG1,SERPINE1 & $217(22)$ \\
\hline FOXA2 & & transcription regulator & & 1,668 & bias & $2,24 \mathrm{E}-03$ & 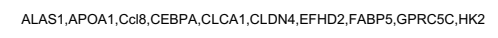 & $111(7)$ \\
\hline mir-21 & & microRNA & Inhibited & $-3,219$ & bias & $2,25 \mathrm{E}-03$ & 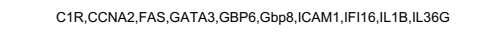 & $184(19)$ \\
\hline FOXL2 & & transcription regulator & Activated & 2,429 & bias & $2,26 \mathrm{E}-03$ & BCL2A1,BMP2,FAS, ICAM1,SOD2, TNFAPP3 & \\
\hline BCL2L1 & & other & & $-0,928$ & & $2,26 E-03$ & CASP3,CCL12,CD44,FAS, L118,NQO 1 & $160(18)$ \\
\hline CASR & & G-protein coupled receptor & & 1,667 & bias & $2,26 \mathrm{E}-03$ & 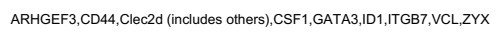 & $132(7)$ \\
\hline TLR8 & & transmembrane receptor & Activated & 2,235 & bias & $2,31 E-03$ & CCR7,СD40,СD83,СD86, IL1B & $146(18)$ \\
\hline A2M & & transporter & & 0,372 & bias & $2,31 E-03$ & CCI2,GADD458, GAP43, L18,LRP1 & $180(20)$ \\
\hline IFNGR1 & & transmembrane receptor & & 1,698 & bias & $2,31 E-03$ & CO12,CXCL12,FAS,ICAM1,L1B & $151(15)$ \\
\hline NLRP3 & & other & & 0,840 & & $2,35 E-03$ & CO12,CSF3, СХCR3, GATA3, LI 1B,MRC1, RELB & 150 (15) \\
\hline bexarotene & & chemical drug & & 1,411 & bias & $2,40 \mathrm{E}-03$ & 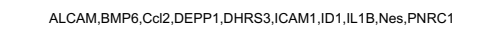 & $193(18)$ \\
\hline cytarabine & & chemical drug & Activated & 2,431 & bias & $2,44 \mathrm{E}-03$ & CD40,CD86,FAS,HLA-A,ICAM1, IL1B & $188(18)$ \\
\hline black raspberry extract & & chemical drug & & & & $2,46 E-03$ & AKR1110,BUB1,CD44,KRT20,LAMB3,PLS3,SDC1,SLC30A1 & \\
\hline RET & & kinase & Activated & 2,425 & bias & $2,46 E-03$ & CCl12,CSF1,CSF3,CXCL12,L118, КLKK,PTPN13,SCARA3 3 & $181(17)$ \\
\hline CCR2 & & G-protein coupled receptor & Activated & 2,743 & bias & $2,46 \mathrm{E}-03$ & $\mathrm{CCl} 2, \mathrm{CCl} 1, \mathrm{CD} 40, \mathrm{CD} 86, \mathrm{CSF} 1, \mathrm{CSF} 3, \mathrm{CXCL12,1118 \textrm {B }}$ & $184(19)$ \\
\hline BRD4 & & kinase & Activated & 2,000 & bias & $2,49 E-03$ & CCNA2,CEBPA,ICAM1,LIRR,LCK,MAL,MCM3,NQO1,STC2 & 159 (11) \\
\hline RORA & & ligand-dependent nuclear receptor & & 1,609 & bias & $2,49 \mathrm{E}-03$ & 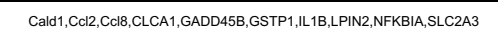 & $131(13)$ \\
\hline aluminum hydroxide & & chemical drug & & & & $2,50 \mathrm{E}-03$ & Glycam1,ICAM1 & \\
\hline
\end{tabular}




\begin{tabular}{|c|c|c|c|c|c|c|c|c|}
\hline LY75 & 1,199 & transmembrane receptor & & & & $2,50 \mathrm{E}-03$ & $\mathrm{CD} 40, \mathrm{CD} 86$ & $97(7)$ \\
\hline B3GNT2 & & enzyme & & & & $2,50 \mathrm{E}-03$ & CD14,L1B & \\
\hline CYP2C8 & & enzyme & & & & $2,50 \mathrm{E}-03$ & $\mathrm{CO} 12,111 \mathrm{~B}$ & $137(11)$ \\
\hline SIRT4 & & enzyme & & & & $2,50 \mathrm{E}-03$ & $\mathrm{CO} 21,111 \mathrm{~B}$ & \\
\hline Casein & & group & & & & $2,50 \mathrm{E}-03$ & ІСАM $1, L 1 B$ & \\
\hline cerotic acid & & chemical - endogenous mammalian & & & & $2,50 \mathrm{E}-03$ & CD40,L1B & \\
\hline RNF6 & & transcription regulator & & & & $2,50 \mathrm{E}-03$ & BMF,KLK3 & $136(7)$ \\
\hline TAOK2 & & kinase & & & & $2,50 \mathrm{E}-03$ & GSTP1,SOD2 & \\
\hline miR-328-3p (and other miRNAs w/seed UGGCCCU) & & mature microRNA & & & & $2,50 \mathrm{E}-03$ & CD44,CEBPA & \\
\hline EGFL7 & & other & & & & $2,50 \mathrm{E}-03$ & ICAM1,NFKBAA & \\
\hline FBXL5 & & enzyme & & & & $2,50 \mathrm{E}-03$ & FTH1,SLC11A2 & \\
\hline PTCH2 & & transmembrane receptor & & & & $2,50 \mathrm{E}-03$ & CXCL12,JAG1 & \\
\hline CFL1 & & other & & & & $2,50 \mathrm{E}-03$ & ICAM1,UUN & $122(12)$ \\
\hline Mis & & other & & & & $2,50 \mathrm{E}-03$ & CEBPD,GSTP1 & \\
\hline DEETGE-CAL-Tat & & chemical reagent & & & & $2,50 \mathrm{E}-03$ & $\mathrm{NQO1}, \mathrm{SOD2}$ & \\
\hline ascomycin & & chemical reagent & & & & $2,50 \mathrm{E}-03$ & $\mathrm{CD83,CD86}$ & \\
\hline phenanthridine & & chemical toxicant & & & & $2,50 \mathrm{E}-03$ & CD83,CD86 & \\
\hline succinylacetone & & chemical - endogenous mammalian & & & & $2,50 \mathrm{E}-03$ & ALAS1, CYBA & \\
\hline $\max$ & & transcription regulator & & $-0,128$ & & $2,51 E-03$ & FTH1,GADD 458,HK2,HMGA1,D1,KLF6,SLC2A3 & \\
\hline COL18A1 & & other & Inhibited & $-2,621$ & bias & $2,51 \mathrm{E}-03$ & ANTXR1,HK2, ICAM1,ID1,ID3,JUN,SERPINE1 & $181(17)$ \\
\hline TCLAA & & transcription regulator & & & & $2,51 \mathrm{E}-03$ & ACOD1,CD68,CLEC4D,GDF $15, \mathrm{LL} 1 \mathrm{1B}, \mathrm{LY} 9, \mathrm{STEAP} 4$ & \\
\hline choline fenofibrate & & chemical drug & & $-0,218$ & & $2,51 \mathrm{E}-03$ & CXCL12,NFKBA,SERPRE 1,UGTAA7 (nincludes others) & $111(7)$ \\
\hline CX3CR1 & & G-protein coupled receptor & & 0,000 & & $2,51 \mathrm{E}-03$ & CO12,CD14,ICAM1,L11B & $160(17)$ \\
\hline RC3H1 & & enzyme & & & & $2,51 E-03$ & CCR7, I1B, LLST,SDC1 & \\
\hline MALP-2s & & chemical reagent & Activated & 2,000 & bias & $2,51 E-03$ & BCL2A, СD40,СD86, L1B & $194(21)$ \\
\hline SNCA & & enzyme & & 1,779 & bias & $2,55 E-03$ & 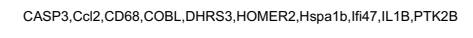 & $201(18)$ \\
\hline VitaminD3-VDR-RXR & & complex & Activated & 2,207 & bias & $2,55 E-03$ & CD14,LLIRL1,KLF4,SEMA3B,SERPINB1 & \\
\hline MYBL2 & & transcription regulator & & 0,152 & bias & $2,55 E-03$ & BIRC3,BUB 1, CCNA2, CEBPA,ID1 & \\
\hline SPHK1 & & kinase & & 1,432 & bias & $2,55 E-03$ & BIRC3,CC12,L118,JUN,SERPINE 1 & $174(18)$ \\
\hline IL12A & & cytokine & & 1,091 & bias & $2,55 E-03$ & CASP3,CD86,FAS, ICAM1, L11B & $160(16)$ \\
\hline vincistine & & chemical drug & & 1,467 & bias & $2,55 E-03$ & FAS,GAP43,IL1B,JUN,SOD2 & $170(20)$ \\
\hline D-fructose & & chemical-endogenous mammalian & Activated & 2,219 & bias & $2,55 E-03$ & CO12,CD68, СЕBPA,ICAM1,L1B & $176(14)$ \\
\hline TAL1 & & transcription regulator & & $-1,066$ & & $2,59 E-03$ & 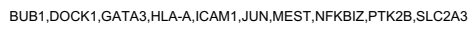 & $131(12)$ \\
\hline Hdac & & group & & $-0,426$ & & $2,61 \mathrm{E}-03$ & BMP2,FOSL1,GADD45B,GATA3,LL18,JUN,JUP,KLF6,PREX1,SMO & $244(22)$ \\
\hline DКK1 & & growth factor & Inhibited & $-2,389$ & bias & $2,63 E-03$ & BMP4,BMP6,CD44, GATA3,RUN×2,TCF & $146(9)$ \\
\hline RHOA & & enzyme & & 1,347 & bias & $2,67 E-03$ & GDF15,ICAM1,LL18,JUN,PRKG1,RND3,RUN×2 & $203(21)$ \\
\hline imatinib & & chemical drug & & $-0,292$ & bias & $2,711-03$ & BLNK,BMP2,CASP3,CCNA2,CEBPA,CXCL12,ID1,L181,LYN,MAPK4 & $222(23)$ \\
\hline
\end{tabular}




\begin{tabular}{|c|c|c|c|c|c|c|c|c|}
\hline Actin & & group & & & & $2,72 E-03$ & CAVIN1,CD44,L1B & \\
\hline Pprerd & & phosphatase & & & & $2,72 E-03$ & COI2,CEBPD,CXCL14 & \\
\hline N(2)-Gamma-D-glutamyl)-meso-2,2-diaminopimelic acid & & chemical reagent & & & & $2,72 E-03$ & Col2,ICAM1,L1B & $152(15)$ \\
\hline elastase & & group & & & & $2,72 E-03$ & ICAM1, IL1B,MLKL & $133(15)$ \\
\hline PSMB10 & 0,446 & peptidase & & & & $2,72 E-03$ & GATA3,NFKBIA,TNFAIP3 & $85(7)$ \\
\hline Betacatenin/TCF & & complex & & & & $2,72 E-03$ & CD44,JUN,RUNX2 & \\
\hline ACE2 & & peptidase & & & & $2,72 E-03$ & COI2,LL1B,SERPINE1 & $160(11)$ \\
\hline Hsp27 & & group & & 0,000 & & $2,80 E-03$ & CASP3,CD14,CD86,,CSF1,KLK3 & $192(18)$ \\
\hline LRP6 & & transmembrane receptor & & $-0,176$ & & $2,80 E-03$ & CD68,GATA3,HK2,L118,SLC2A3 & \\
\hline ADRA1B & & G-protein coupled receptor & & & & $2,80 \mathrm{E}-03$ & CEBPD,FABP5, ILGST,JUN,RALA & $118(10)$ \\
\hline TCF7 & & transcription regulator & & & & $2,80 \mathrm{E}-03$ & СЕВРА,СЕВРD, GАТАЗ,LСK,ТСТ & $123(9)$ \\
\hline $\operatorname{lgm}$ & & complex & & $-1,119$ & & $2,84 E-03$ & BCL2AA, ,CCNA2, CD40, CD44, FAS, ID3 & $179(20)$ \\
\hline SPDEF & & transcription regulator & & & & $2,84 E-03$ & BIRC3,СD40,KLKЗ3,LASP1,SDC1,SERPINE1 & $100(9)$ \\
\hline RASSF1 & & other & & 0,626 & & $2,85 E-03$ & AMIGO2,CASP3,CAV2, CCNA2,GDF 15,KLF4,LLTAF & $137(7)$ \\
\hline SAMSN1 & & other & Activated & 2,646 & bias & $2,85 E-03$ & BATF,CD40,NFKBIZ,OASL,SDC1,Vnn3,ZC3H12A & $89(6)$ \\
\hline CLEC7A & & transmembrane receptor & & 1,940 & bias & $2,87 E-03$ & CCR7,CLCA1,CSF3,ІL1B & $164(18)$ \\
\hline B2M & & transmembrane receptor & & 0,152 & bias & $2,87 \mathrm{E}-03$ & CD8A,Hamp/Hamp2,HLA-A,L1B & \\
\hline FCGR2A & & transmembrane receptor & & 1,992 & bias & $2,87 \mathrm{E}-03$ & COC2,FAS,IL18,SERPINE1 & $205(20)$ \\
\hline oltipraz & & chemical drug & & 1,961 & bias & $2,87 \mathrm{E}-03$ & FTH1,GSTP1,NQO1,UGT1A7 (includes others) & \\
\hline methylnitronitrosoguanidine & & chemical toxicant & & 1,960 & bias & $2,87 E-03$ & CEBPA,FAS,GDF15,SERPINE1 & $204(17)$ \\
\hline suramin & & chemical drug & & $-0,911$ & & $2,87 \mathrm{E}-03$ & CO12,CD40, СD86,ICAM1 & $150(18)$ \\
\hline dicarbethoxydihydrocollidine & & chemical toxicant & & & & $2,87 \mathrm{E}-03$ & COL2,CCNA2,I118,KRT19 & $81(7)$ \\
\hline hemozoin & & hremical- endogenous non-mammalia & & 1,921 & bias & $2,87 \mathrm{E}-03$ & CC12,CD83,ICAM1,L11B & $154(18)$ \\
\hline CPG ODN 2006 & & chemical reagent & Activated & 2,507 & bias & 3,03E-03 & BIRC3,CD40,CD886, ICAM1, I1 18,NFKBBZ,OASL & 146 (16) \\
\hline MIF & & cytokine & Activated & 2,742 & bias & 3,05E-03 & CO12,CD44,CXCL16,ICAM1, IL18,IL6ST, IL7RR,JUN & $184(20)$ \\
\hline paraquat & & chemical toxicant & & 1,916 & bias & $3,05 E-03$ & APOA1,CEBPD,FAS,GSTA3,GSTP1,KLF6,LAMC2,LPIN2 & $136(7)$ \\
\hline сз & & peptidase & & 1,378 & bias & $3,06 E-03$ & CASP3,CCI2,CSF1,CXCL16,IL18,UUN & $143(16)$ \\
\hline MYRF & & transcription regulator & & 0,000 & & $3,06 E-03$ & ANXAG,CFI,GDF 15,HPGDS,MFSD1,RHOU & \\
\hline PRKD1 & & kinase & Activated & 2,190 & bias & $3,08 E-03$ & CO12,CD2,CD86,LL1B,TNFAIP3 & $204(21)$ \\
\hline valsartan & & chemical drug & Inhibited & $-2,202$ & bias & $3,08 E-03$ & CCl2,CYBA,ICAM1,IL1B,SERPINE1 & $175(18)$ \\
\hline РTH & & other & Activated & 2,296 & bias & $3,09 E-03$ & BMP2,CI2,CSF1,CXCL12,CYBA,,GALNT3,JUN,RUNX2,Saa3,SDC4 & $154(16)$ \\
\hline EDN1 & & cytokine & Activated & 2,748 & bias & $3,15 E-03$ & ADAM19,ANXAG,BCLLA1,CCR7,FOSL1,1CAM1,JUN,PLCB4,SDC1,SERPINE1 & 176 (16) \\
\hline IFN Beta & & group & & 1,445 & bias & $3,16 \mathrm{E}-03$ & ACOD1,CCR7,CD40,CD83,CD86, HLA-A, IF1616,L118,OASL & $173(18)$ \\
\hline mir-155 & & microRNA & & $-0,684$ & bias & $3,22 E-03$ & 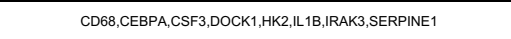 & $201(20)$ \\
\hline HNF1B & & transcription regulator & & 0,816 & & $3,22 E-03$ & CD44,F11R,ID1,ID3,JAG1,RNASE4,UGT1A7 (includes others),UPP1 & \\
\hline RPSA & & translation regulator & Inhibited & $-2,000$ & bias & $3,26 \mathrm{E}-03$ & CSF3,If47,IL1B,RELB & $151(9)$ \\
\hline mir-221-3p (and other miRNAs w/sed GCUACAU) & & mature microRNA & & $-1,994$ & bias & $3,26 \mathrm{E}-03$ & ВMF,ІІАМM1,КККЗ,SOD2 & \\
\hline
\end{tabular}




\begin{tabular}{|c|c|c|c|c|c|c|c|}
\hline vav3 & cyokine & & & & 3,26E-03 & BCL2A1, CAV2,KLK3,RALA & \\
\hline CDK5 & kinase & & 1,929 & bias & $3,26 \mathrm{E}-03$ & CD14,JUN,RALA,TNFAIP3 & $113(10)$ \\
\hline NFATC3 & transcription regulator & & $-0,254$ & bias & 3,29E-03 & B3GAT1,BMP2, САSP3, GAP44,JUN,PRKD3 & \\
\hline 2-deoxyglucose & chemical drug & & 0,056 & & $3,29 E-03$ & BMF,САSP, CCI2,L118,UUN,SOD2 & $164(17)$ \\
\hline AKT1 & kinase & Activated & 2,751 & bias & 3,29E-03 & 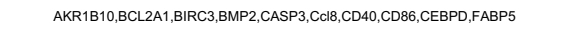 & 209 (17) \\
\hline monophosphoyll lipid A & chemical drug & & & & $3,34 E-03$ & $\mathrm{CCl2,CD86,L1 \textrm {BB }}$ & $155(20)$ \\
\hline 1"-acetoxychavicol acetate & chemical reagent & & & & $3,34 \mathrm{E}-03$ & BCLLA1,BRC3,ICAM1 & $118(12)$ \\
\hline TIFA & other & & & & 3,34E-03 & ICAM1,NFKBIA,TNFAIP3 & 126 (15) \\
\hline NSD1 & transcription regulator & & & & $3,34 \mathrm{E}-03$ & BMP4,IRAK3,KLFF & \\
\hline TRAF5 & transporter & & & & $3,34 E-03$ & CD86,FAS,ICAM1 & 155 (17) \\
\hline TNFSF9 & cytokine & & & & $3,34 \mathrm{E}-03$ & CCR7,CD14,ICAM1 & $104(7)$ \\
\hline z-551 & chemical reagent & & & & $3,34 \mathrm{E}-03$ & $\mathrm{CCl} 2, \mathrm{CD} 68, \mathrm{IL} 1 \mathrm{~B}$ & $105(7)$ \\
\hline FITC & chemical reagent & & & & $3,34 E-03$ & CCl2,CDB6,ICAM1 & \\
\hline HDL & complex & Inhibited & $-2,159$ & bias & 3,37E-03 & CCI2,CD40,CD86,ICAM1, LL1B & $147(18)$ \\
\hline PDLIM2 & other & & $-1,890$ & & $3,41 E-03$ & BST1,CRISPLD2,FBP1,RTN1, SCARA3,SLC43A3,STC2 & \\
\hline mir-210 & microRNA & Inhibited & $-2,368$ & bias & 3,41E-03 & CHD9, CXCL12,FGFRL1,KLFF, LASP1,RAD52,SCARA3 & \\
\hline Fox04 & transcription regulator & & 0,692 & & $3,41 E-03$ & ANXA3,FABP5,GADD45B,JAG1,LCP2,SERPINE1,SOD2 & 150 (7) \\
\hline ciprofobrate & chemical drug & & 0,174 & & $3,47 \mathrm{E}-03$ & CXCL12,GSTP1,KRT, MGLL,NFKBAA,OAT,Saa3,SLCAAA,Vnn3 & $114(8)$ \\
\hline L-triiodothyronine & chemical-endogenous mammalian & & $-0,730$ & bias & 3,49E-03 & АРОA1,BMP4,CAMK4,СD44,CEBPA,CXCL12,FAS,GDF15, H6PD, ID1 & $190(19)$ \\
\hline LGALS1 & other & & 1,710 & bias & 3,53E-03 & ANG,COL2,CXCL16, IFNGR2,L1L 18,SERPINE1 & $219(23)$ \\
\hline GW3965 & chemical reagent & & $-1,039$ & & $3,57 E-03$ & 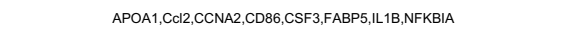 & 148 (12) \\
\hline RPTOR & other & & $-0,136$ & bias & 3,62E-03 & 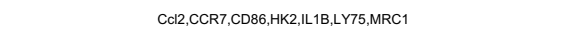 & 188 (21) \\
\hline TIRAP & other & & 0,762 & bias & $3,68 \mathrm{E}-03$ & CD40, СС86,ICAM, LL1B & 148 (17) \\
\hline VTN & other & & 1,199 & bias & 3,68E-03 & IL1B,RUNX2,SERPINE1,SFN & $154(20)$ \\
\hline TAB1 & enzyme & & $-0,762$ & bias & $3,68 E-03$ & BIRC3,LL1B,JUN,SERPINE1 & $143(18)$ \\
\hline butylated hydroxyanisol & chemical toxicant & & 1,896 & bias & $3,68 E-03$ & GSTA3,GSTP1,JUN,NQO1 & $46(3)$ \\
\hline selenium & chemical drug & & $-0,152$ & & $3,68 \mathrm{E}-03$ & APOA1,СЕВРА,КLKЗ,МRC1 & $114(7)$ \\
\hline Hedgehog & group & & & & $3,68 E-03$ & CCNA2,JAG1,KLF4,KLKK3,KRT19 & \\
\hline salicylic acid & chemical drug & & $-1,000$ & bias & $3,68 E-03$ & APOA 1, ,CF 1, CSF 3, , DF 15 , IL1B & $161(20)$ \\
\hline AMPK & complex & & 1,195 & & $3,75 E-03$ & BMF,BMP2,CC12,CXCL12,LITAF,OAT,RUNX2,SERPINE1 & $202(21)$ \\
\hline 3M-002 & chemical reagent & Activated & 2,204 & & $3,75 E-03$ & 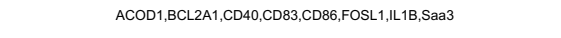 & $140(15)$ \\
\hline staurosporine & chemical -kinase inhibitor & & $-1,238$ & & $3,75 E-03$ & CASP3,CD28,FAS,FOSL1,1CAM1, LL 18,NQ01, SERPINE1 & $169(20)$ \\
\hline rifampin & chemical drug & Activated & 2,412 & bias & $3,79 E-03$ & 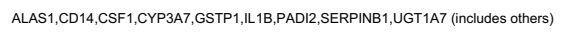 & 26 (4) \\
\hline H89 & chemical - kinase inhibitor & & $-1,795$ & bias & $3,79 E-03$ & CO12,CD14,CD83,,GSTP1,1L18,NFKBA,,SDC1, SERPINE1,SOD2 & 209 (16) \\
\hline daidzein & chemical drug & & 0,816 & bias & $3,84 E-03$ & CASP3,СD86, СXCR3,HLA-A,LCP2,NQO1,RASGRP1 & 169 (17) \\
\hline ARNT & transcription regulator & & 0,277 & bias & $3,95 E-03$ & CCR7,CD81, CSF3,CXCR3,HK2,NQO1,SERPINE1,SLC2A3 & $182(13)$ \\
\hline vorinostat & chemical drug & & $-1,356$ & & 3,99E-03 & 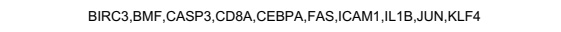 & $204(18)$ \\
\hline
\end{tabular}




\begin{tabular}{|c|c|c|c|c|c|c|}
\hline 9,10-dimethy-1,2-benzanthracene & chemical toxicant & & & $4,02 E-03$ & LL18,JUN,MRC1,NQO1,SERPINE1 & $182(20)$ \\
\hline beta-naphthoflavone & chemical toxicant & 1,350 & bias & $4,02 E-03$ & FTH1,GSTP1,NQ01,Saa3,UGT1A7 (includes others) & 208 (17) \\
\hline HDAC5 & transcription regulator & & & $4,05 E-03$ & HK2,HMGCS2,JUN,KLFG,PTPN13,RUNX2 & \\
\hline AGER & transmembrane receptor & 1,449 & bias & $4,05 E-03$ & CCI2,CSF1,FAS, ICAM1, LL 18,SERPINE1 & $186(21)$ \\
\hline SHARPIN & other & & & $4,05 E-03$ & IL1B,MLKL,TNFAIP3 & $120(14)$ \\
\hline GAS2L3 & other & & & 4,05E-03 & FAS,PTPN14,TM4SF1 & \\
\hline ВМР10 & growth factor & & & $4,05 E-03$ & ICAM1,ID1,NFKBIA & 149 (7) \\
\hline PRF1 & transporter & & & $4,05 E-03$ & 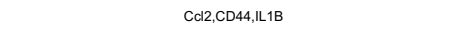 & $137(12)$ \\
\hline MAFG & transcription regulator & & & $4,05 E-03$ & GSTA3,GSTP1,NQO1 & \\
\hline EPHX2 & enzyme & & & $4,05 E-03$ & COI2,ICAM1, IL1B & $190(19)$ \\
\hline MAPK10 & kinase & & & $4,05 E-03$ & CASP3,CD44,JUN & $184(17)$ \\
\hline CxCL2 & cytokine & & & 4,05E-03 & COI2,ICAM1,IL1B & $182(18)$ \\
\hline VIPR1 & G-protein coupled receptor & & & $4,05 E-03$ & $\mathrm{CCl} 2, \mathrm{CD} 40, \mathrm{CD} 86$ & $119(13)$ \\
\hline SMURF2 & enzyme & & & $4,05 E-03$ & JUN,RUNX2,SERPINE1 & 186 (17) \\
\hline MAML1 & transcription regulator & & & $4,05 E-03$ & LL1B,IL7R,RND3 & $66(7)$ \\
\hline EFNB2 & kinase & & & 4,05E-03 & ILTR,RUNX2,TAM1 & \\
\hline DSP & other & & & 4,05E-03 & COI2,CEBPA,JUP & \\
\hline BHLHA15 & transcription regulator & & & $4,05 E-03$ & CCKAR,GJBz,KRT19 & \\
\hline cyclopiazonic acid & hemical - endogenous non-mammaila & & & 4,05E-03 & BCL2A1,CC12,FAS & $86(7)$ \\
\hline silicon dioxide & chemical drug & & & $4,05 E-03$ & FOSL1,LL1B,JUN & $208(21)$ \\
\hline erythromycin & chemical drug & & & 4,05E-03 & CD86,ICAM1,IL1B & 148 (17) \\
\hline CDK19 & kinase & $-1,414$ & bias & $4,07 E-03$ & CD81,FAS,JUN,RHBDF2,SDC1,SFN,UPP 1 & \\
\hline atorvastatin & chemical drug & $-1,671$ & & $4,09 E-03$ & 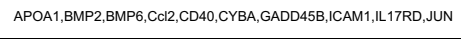 & $204(21)$ \\
\hline p38 Sapk & group & & & $4,11 E-03$ & $\mathrm{CD83,CD86}$ & \\
\hline Mstkrs & group & & & $4,11 E-03$ & RELB,YAP1 & \\
\hline ATPase & group & & & $4,11 E-03$ & CD40,ICAM1 & \\
\hline Em & group & & & $4,11 E-03$ & CD44,ICAM1 & \\
\hline cYp & group & & & $4,11 E-03$ & CD86,NQ01 & \\
\hline olmesartan & chemical drug & & & $4,11 \mathrm{E}-03$ & CYBA,ICAM1 & \\
\hline Foхk2 & transcription regulator & & & 4,11E-03 & MCM3,SERPINE1 & \\
\hline ZMIZ2 & transcription regulator & & & $4,11 \mathrm{E}-03$ & CD44,JUN & \\
\hline Mucin & group & & & 4,11E-03 & CD83,СD86 & \\
\hline DCB 3503 & chemical reagent & & & $4,11 E-03$ & $\mathrm{CO} 12,111 \mathrm{~B}$ & \\
\hline TDP2 & transcription regulator & & & $4,11 E-03$ & КடКз,SYT1 & \\
\hline HADHA & enzyme & & & 4,11E-03 & $\mathrm{CO} 12,11 \mathrm{BB}$ & $95(7)$ \\
\hline MAPKAPK5 & kinase & & & $4,11 E-03$ & MAPKG,TNFAPP3 & \\
\hline Orm1 (includes others) & other & & & 4,11E-03 & CO12,L11B & \\
\hline
\end{tabular}




\begin{tabular}{|c|c|c|c|c|c|c|c|}
\hline EIF2AK1 & kinase & & & & $4,11 \mathrm{E}-03$ & Hamp/Hamp2,L1B B & \\
\hline BCAR3 & other & & & & $4,11 E-03$ & RALA,RRAS & \\
\hline CES1 & enzyme & & & & $4,11 E-03$ & $\mathrm{CO} 12,11 \mathrm{BB}$ & \\
\hline Cxc13 & cytokine & & & & $4,11 E-03$ & СЕВРA,СЕВPD & \\
\hline ELF2 & transcription regulator & & & & $4,11 E-03$ & КடКз,LYN & \\
\hline 4,4-d-disothiocyanostilbene-2,2-disulfonic acid & chemical drug & & & & $4,11 E-03$ & CASP3,CO12 & $128(13)$ \\
\hline cimetidine & chemical drug & & & & $4,11 E-03$ & FAS,GDF15 & \\
\hline cinnamy-3,4-dihydroxy-alpha-cyanocinnamate & chemical reagent & & & & $4,11 E-03$ & $\mathrm{COL} 2, \mathrm{MRC} 1$ & \\
\hline orantinib & chemical drug & & & & $4,11 \mathrm{E}-03$ & CASPB,ICAM1 & \\
\hline retinaldehyde & chemical-endogenous mammalian & & & & 4,11E-03 & BMP2,CD44 & \\
\hline Stat3-Stat3 & complex & Activated & 2,000 & bias & $4,14 E-03$ & FTL,ICAM1,JUN,SOD2 & $144(7)$ \\
\hline Hохс8 & transcription regulator & & $-1,000$ & & $4,14 \mathrm{E}-03$ & ADAM19,CC12,KRT19,NQO1 & \\
\hline SHH & peptidase & & 1,133 & bias & $4,28 E-03$ & 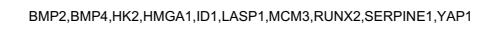 & $213(26)$ \\
\hline miR-199a-5p (and other miRNAs w/seed CCAGUGU) & mature microRNA & Inhibited & $-2,433$ & bias & $4,33 E-03$ & ID3,JAG1,KLF4,LAMC2,RND1,ZBTB16 & \\
\hline methamphetamine & chemical drug & Activated & 2,407 & bias & $4,33 E-03$ & COI2,FAS,HOMER2,ICAM1, IL18, JUN & $200(18)$ \\
\hline PELP1 & other & & & & 4,35E-03 & 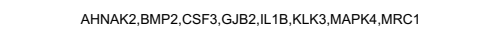 & $121(7)$ \\
\hline GPER1 & G-protein coupled receptor & Activated & 2,236 & bias & $4,37 E-03$ & CEBPD,JUN,NFKBA,RND1,TNFAIP3 & 259 (21) \\
\hline enalapril & biologic drug & & 0,447 & & $4,37 E-03$ & CLDN4,CP,SERPINE1,SLC3AA2,TNFRSF12A & \\
\hline RB1 & transcription regulator & & $-0,473$ & & $4,41 E-03$ & 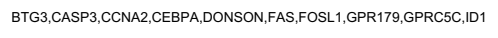 & $215(20)$ \\
\hline Pdgf (complex) & complex & & 1,424 & bias & $4,55 E-03$ & COL12,CD44,CYBA, ICAM1,JAG1,JUN,SERPINE1 & ${ }^{197(20)}$ \\
\hline NTRK2 & kinase & & 1,964 & bias & 4,63E-03 & BMP2,CCNA2,JAG1,MUC1,SERPINE1,STT1 & $97(7)$ \\
\hline кDМзА & transcription regulator & & 1,982 & bias & 4,63E-03 & ALCAM, CD9, GDF15,SERPINE1 & \\
\hline ARHGAP31 & other & & 0,000 & & $4,63 E-03$ & BMP2,GADD45B,GDF15,JUN & \\
\hline sost & other & & 1,980 & bias & 4,63E-03 & COL2, CEBPA,L118,SOD2 & 129 (12) \\
\hline Сүвв & enzyme & Activated & 2,000 & bias & $4,63 E-03$ & COL2,CYBA,ICAM1,L11B & 195 (17) \\
\hline LLTRA & transmembrane receptor & & 1,992 & bias & $4,63 E-03$ & 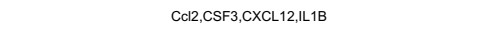 & $153(15)$ \\
\hline SFRP1 & transmembrane receptor & & $-1,413$ & bias & $4,74 \mathrm{E}-03$ & CASP3,GATA3,RUNX2,SERPINE1,TCFT & $189(16)$ \\
\hline MMP1 & peptidase & & 1,432 & bias & $4,74 E-03$ & CXCL12,JUN,NFKBAA,RUNX2,SERPINE1 & $152(15)$ \\
\hline $\mathrm{DCN}$ & other & & $-0,836$ & & $4,74 E-03$ & CASP 3, ICAM1, IL1B,SDC 1, SERPINE 1 & ${ }^{167(23)}$ \\
\hline IL12 (family) & group & Activated & 2,813 & bias & $4,79 E-03$ & ADAM19,CD44,CD86,,GATA3,L1 18,NFKBIZ,SERPINB 1,SLC2A3 & $185(20)$ \\
\hline FZD9 & G-protein coupled receptor & & & & $4,85 E-03$ & CC12,CXCL14,F1616 & \\
\hline fontolizumab & biologic drug & & & & $4,85 E-03$ & FAS,LAG3,TGM3 & \\
\hline GIP & other & & & & $4,85 E-03$ & 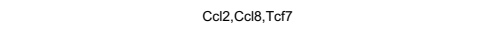 & \\
\hline CD70 & cytokine & & & & $4,85 E-03$ & BCL2A1,CD44,FAS & $140(19)$ \\
\hline LLRAP & transmembrane receptor & & & & $4,85 E-03$ & CD14,CD40,LLRL1 & $134(10)$ \\
\hline SIPR2 & G-protein coupled receptor & & & & $4,85 E-03$ & CCI2,ICAM1,JUN & ${ }^{197}(20)$ \\
\hline КLK5 & peptidase & & & & $4,85 E-03$ & CCl8,ICAM1,IL1B & \\
\hline
\end{tabular}




\begin{tabular}{|c|c|c|c|c|c|c|c|}
\hline 7-nitroindazole & chemical reagent & & & & $4,85 E-03$ & GSTP1,NFKBA,PRKG1 & \\
\hline pheny-N-Nert-butylintrone & chemical reagent & & & & $4,85 E-03$ & $\mathrm{CD} 40, \mathrm{CD} 83, \mathrm{CD} 86$ & $169(14)$ \\
\hline glutamy-Se-methylselenocysteine & chemical-endogenous mammalian & & & & $4,85 E-03$ & GSTP1,ID1,JUN & \\
\hline 113 & cytokine & & & & $4,94 \mathrm{E}-03$ & ADAM19,CD14,CD68,,CSF1,CSF3,FAS & $165(19)$ \\
\hline MAP2KG & kinase & & $-0,763$ & bias & 4,94E-03 & CO12,CD40, СD83, CD86,JUN, RELB & 138 (17) \\
\hline doxycycline & chemical drug & & 1,000 & & 4,94E-03 & BMP2,СD86,CXCL12,SDC1,SOD2,XDH & $127(13)$ \\
\hline ESRRA & ligand-dependent nuclear receptor & & 1,562 & bias & $4,99 E-03$ & 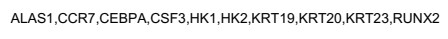 & $80(5)$ \\
\hline acetaminophen & chemical drug & Activated & 2,394 & bias & $4,99 E-03$ & 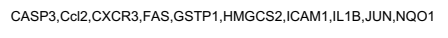 & $187(19)$ \\
\hline diethylnitrosamine & chemical toxicant & & 0,555 & & $5,07 E-03$ & CASP3,CXCL12,GSTP1,Hspa 1b,KRT8, OAT,Vחn3 & $136(7)$ \\
\hline L-methionine & chemical-endogenous mammalian & & & & $5,14 E-03$ & BUB1,GADD45B,IF16,NFKB|A,SOD2 & $143(7)$ \\
\hline CASPB & peptidase & & & & $5,14 E-03$ & CD14,CD44,CSF1,FAS,IL1B & 139 (14) \\
\hline LPL & enzyme & & 1,792 & bias & $5,14 \mathrm{E}-03$ & APOA 1, CASP3, СSF 1, ,CAM 1,1118 & $208(20)$ \\
\hline МАРЗК1 & kinase & Activated & 2,176 & bias & $5,14 E-03$ & BIRC3,FAS,HSPB1,JUN,SERPINE1 & $146(18)$ \\
\hline RGS10 & other & & & & $5,14 E-03$ & CClB,CXCR3,FAS, LL1B,LLSTT & $121(10)$ \\
\hline DETA-NONOate & chemical reagent & & 0,365 & & $5,14 E-03$ & BIRC3,FAS,ICAM1,PRKG1,SERPINE1 & $202(23)$ \\
\hline ARRB2 2 & other & & $-1,987$ & & $5,16 \mathrm{E}-03$ & CC12,L11, NFKBBZ,SERPINE1 & $182(18)$ \\
\hline MARK2 & kinase & & 1,000 & bias & $5,16 \mathrm{E}-03$ & BATF,CD44,NFKBIZ,Vnn3 & \\
\hline green tea polyphenol & chemical drug & & $-1,067$ & bias & $5,16 \mathrm{E}-03$ & 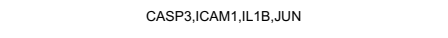 & 139 (15) \\
\hline barddoxolone & chemical drug & & 1,067 & bias & $5,16 \mathrm{E}-03$ & CEBPA,FTH1,NFKBAA,NQ01 & \\
\hline PD173074 & chemical reagent & Activated & 2,449 & bias & $5,27 E-03$ & CCR7,CEBPA,DEPP1,GATA3,KRT23,PRR5 & \\
\hline kanini acid & chemical toxicant & Activated & 2,919 & bias & $5,35 E-03$ & CASP3,,C121,FOSL1,GADD45B,GAP43,L118,JUN,SDC1,SLC39A4 & 169 (18) \\
\hline LIRN & cytokine & Inhibitied & $-2,179$ & bias & 5,52E-03 & CD44,HGPD,ICAM1,IL18, KLFF, OASL,RIPK2,SERPINE1 & 175 (19) \\
\hline MOG & other & & & & $5,56 \mathrm{E}-03$ & 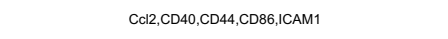 & 156 (16) \\
\hline miR-34a-5p (and other miRNAs w/seed GGCAGUG) & mature microRNA & Inhibited & $-2,215$ & bias & $5,56 \mathrm{E}-03$ & ICAM1,JAG1,KLF4,MCM3,NOTCH2 & \\
\hline BNIP3L & other & & 0,000 & bias & $5,56 E-03$ & BLNK,CCL2,CCNAZ,NFKBIA,PTK2B & $110(11)$ \\
\hline bicuculline & hemical - endogenous non-mammalia & Activated & 2,191 & bias & $5,56 \mathrm{E}-03$ & GADD 45B, HK2,JUN,KLF4,SLCZA3 & $162(12)$ \\
\hline PRKCE & kinase & & 1,633 & bias & $5,61 \mathrm{E}-03$ & AMIG02,BIRC3,CSF3,IL18, ILLGT,JUN & $164(17)$ \\
\hline cCL5 & cytokine & Activated & 2,416 & bias & $5,61 \mathrm{E}-03$ & ALCAM, $, \mathrm{Cl} 12, \mathrm{CD} 40, \mathrm{CD} 44, \mathrm{HMGA} 1, \mathrm{L1} 1 \mathrm{~B}$ & $166(18)$ \\
\hline 1-methyl-4-pheny-1,2,3,6-tetrahydyropyridine & chemical toxicant & Activated & 2,180 & bias & $5,61 \mathrm{E}-03$ & CASP3,CC12,HSPB1,L11 18,JUN,SOD2 & 171 (15) \\
\hline rottlerin & chemical toxicant & Inhibited & $-2,415$ & bias & $5,61 E-03$ & АРОВЕСЗ3,BIRC3,CCL12,CLDA4,ICAM1,JUN & $195(18)$ \\
\hline Citp & group & & & & $5,73 \mathrm{E}-03$ & GATA3,JUP,PERP & \\
\hline IFNW1 & cyokine & & & & $5,73 \mathrm{E}-03$ & $\mathrm{CD} 40, \mathrm{CD} 83, \mathrm{CD} 86$ & \\
\hline GLIS2 & transcription regulator & & & & 5,73E-03 & CO12,LTBP2,SERPINE1 & \\
\hline s100B & other & & & & $5,73 E-03$ & CC12,1118,JUN & 177 (19) \\
\hline TAF1 & transcription regulator & & & & 5,73E-03 & BCL2A1,CCNA2,RUNX2 & $135(7)$ \\
\hline PLA2G4A & enzyme & & & & 5,73E-03 & COL2,ICAM1,L1B & $154(16)$ \\
\hline cxсL3 & cytokine & & & & 5,73E-03 & CCl2,ICAM1,IL1B & 199 (19) \\
\hline
\end{tabular}




\begin{tabular}{|c|c|c|c|c|c|c|c|c|}
\hline EGLN3 & & enzyme & & & & 5,73E-03 & CC12,HamplHamp2,11818 & 149 (7) \\
\hline ACTN4 & & transcription regulator & & & & 5,73E-03 & M1B,PDLIM1,SERPINE1 & $115(9)$ \\
\hline propionic acid & & chemical-endogenous mammalian & & & & 5,73E-03 & CCR7,ICAM1,LL1B & \\
\hline lisinopril & & biologic drug & & & & 5,73E-03 & COC2,L118,JUN & $165(14)$ \\
\hline ZC3H12A & 1,270 & enzyme & & 0,105 & bias & 5,74E-03 & COI2,CEBPA,CEBPD, IL1B & $171(15)$ \\
\hline su6656 & & chemical toxicant & & & & 5,74E-03 & CO12,CSF, 1,LRLL1,KRT8 & $166(15)$ \\
\hline nicotinic acid & & chemical-endogenous mammalian & & $-1,192$ & & 5,74E-03 & ANG,APOA, CD68, IL1B & $156(15)$ \\
\hline sodium orthovanadate & & chemical reagent & & 1,000 & bias & $5,74 E-03$ & CCNA2,CEBPA,NFKBIA,SERPINE1 & $201(22)$ \\
\hline HSF1 & & transcription regulator & & 0,797 & & $5,80 E-03$ & 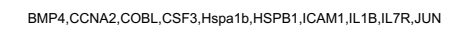 & $207(17)$ \\
\hline THRB & & ligand-dependent nuclear receptor & & $-0,113$ & & $5,80 E-03$ & APOA1, CCI2, CEBPA,FOSL1,1D1, JUN,PGD, RUN 22, SERPINE1,TCEA3 & $203(22)$ \\
\hline fenretinide & & chemical drug & & $-0,651$ & & $5,97 E-03$ & BCL2A, ,CASP, ,L118,JUN,MAPKG,NFKBIA & $164(15)$ \\
\hline MED1 & & transcription regulator & & & & 6,04E-03 & 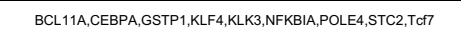 & $203(15)$ \\
\hline WNT1 & & cytokine & Activated & 2,016 & bias & 6,04E-03 & BMPA, СEBPA,JUN,KRT19,RHOU,RUN 2, SERPPNE1,SERPINE2 & $200(20)$ \\
\hline psychosine & & chemical-endogenous mammalian & & & & $6,08 E-03$ & FOSL1,LL1B & $213(23)$ \\
\hline SERCA & & group & & & & $6,08 E-03$ & FOSL1,JUN & $174(15)$ \\
\hline N-hexanoyhomoserine lactone & & hemical - endogenous non-mammalia & & & & $6,08 E-03$ & CSF1,L1B & \\
\hline CCAR1 & & transcription regulator & & & & $6,08 E-03$ & CEBPA,CEBPD & \\
\hline CCN6 & & growth factor & & & & $6,08 E-03$ & BMP4,ICAM1 & \\
\hline $\mathrm{Cr} 3$ & & complex & & & & $6,08 E-03$ & $\mathrm{CD} 40, \mathrm{CD} 86$ & $100(7)$ \\
\hline PXN & & other & & & & $6,08 E-03$ & Кடкз,VСL & $136(7)$ \\
\hline CSRP3 & & other & & & & $6,08 E-03$ & DMD,KRT8 & \\
\hline SDC4 & 0,444 & other & & & & $6,08 E-03$ & แL1B,KLF4 & $104(7)$ \\
\hline W-54011 & & chemical reagent & & & & $6,08 E-03$ & CD86,IL1B & \\
\hline PTPRR & & phosphatase & & & & $6,08 E-03$ & FOSLL1,UN & \\
\hline PIP5K1B & & kinase & & & & $6,08 E-03$ & М18,PРІ5К1C & \\
\hline FGF18 & & growth factor & & & & $6,08 E-03$ & CSF1,RUN $\times 2$ & \\
\hline STX2 & & transporter & & & & $6,08 E-03$ & BMP2,BMP4 & 130 (7) \\
\hline ABCB7 & & transporter & & & & $6,08 E-03$ & FTH1,FTL & $36(3)$ \\
\hline SERPIND1 & & other & & & & $6,08 E-03$ & $\mathrm{CC12,1118}$ & $108(7)$ \\
\hline ferric nitrilotriacetate & & chemical toxicant & & & & $6,08 E-03$ & ID3,KLF4 & \\
\hline FC. 99 & & chemical reagent & & & & $6,08 E-03$ & CD40,CD86 & $106(7)$ \\
\hline calcium chloride & & chemical drug & & & & $6,08 E-03$ & ENPP1,1D3 & \\
\hline ferric chloride & & chemical toxicant & & & & $6,08 E-03$ & FTH1,FTL & \\
\hline phenylamil & & chemical reagent & & & & $6,08 E-03$ & ID1,RUNX2 & \\
\hline zofenopril & & chemical drug & & & & $6,08 E-03$ & $\mathrm{COl1}, \mathrm{CSF} 1$ & \\
\hline NFATC1 & & transcription regulator & & & & $6,26 \mathrm{E}-03$ & 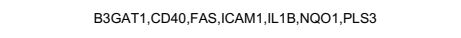 & $187(21)$ \\
\hline lipoxin A4 & & chenical - endogenous mammalian & & $-1,982$ & bias & $6,35 E-03$ & COI2,ICAM1,LL18,SERPINE1 & 198 (21) \\
\hline
\end{tabular}




\begin{tabular}{|c|c|c|c|c|c|c|c|}
\hline 3-methyladenine & chemical toxicant & & 0,334 & & 6,35E-03 & CD40,CD86, I1 11,RUN $\times 2$ & ${ }^{187(21)}$ \\
\hline asopinsilil & chemical drug & & $-1,000$ & bias & 6,35E-03 & CASPB, LAMB3, SFN,XOH & \\
\hline amiodarone & chemical drug & & & & 6,35E-03 & HPN,ICAM1,SLC2A3,SOD2 & \\
\hline NCOR2 & transcription regulator & & & & $6,47 E-03$ & BIRC3,CEBPA,IL18,JUN,RELB & $180(17)$ \\
\hline SERPINF1 & other & & $-0,600$ & & $6,47 E-03$ & BCL2A1,CEBPA,CYBA,NFKBA,,SOD2 & $208(20)$ \\
\hline Iuteolin & chemical drug & & 0,492 & bias & $6,47 E-03$ & CASP3,FAS,ILAM1,JUN,SOD2 & $184(19)$ \\
\hline EGR2 & transcription regulator & & 1,823 & bias & 6,54E-03 & 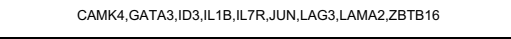 & $155(13)$ \\
\hline potassium chloride & chemical drug & & 1,067 & bias & $6,59 \mathrm{E}-03$ & 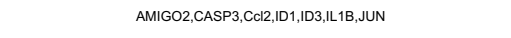 & $183(19)$ \\
\hline THRA & ligand-dependent tuclear r receptor & & 0,577 & & $6,61 E-03$ & APOA 1, BMPA, CAMK4, CCI2, CD 44, CEBPA,JUN,SERPINE 1 & $184(25)$ \\
\hline Pde4 & group & & & & $6,70 E-03$ & CD14,CD40,ICAM1 & 129 (12) \\
\hline CDK5R1 & kinase & & & & $6,70 E-03$ & CD14,LL18,SERPINE1 & $180(14)$ \\
\hline TNFRSF11B & transmembrane receptor & & & & $6,70 E-03$ & BMP2,CC12,RUNX2 & $118(9)$ \\
\hline ibrutinib & chemical drug & & & & $6,70 E-03$ & CD44,CD86,FAS & $136(16)$ \\
\hline NTSE & phosphatase & & & & $6,70 E-03$ & COC1,LL1B,SERPINE1 & $175(21)$ \\
\hline PDCD4 & other & & & & $6,70 E-03$ & CASP3,LL1B,JUN & $199(18)$ \\
\hline RARRES2 2 & transmembrane receptor & & & & $6,70 E-03$ & CCl2,CCNA2,1118 & $61(4)$ \\
\hline Ps-1145 & chemical - kinase inhibitor & & & & $6,70 E-03$ & ICAM1,NFKBA,TNFAIP3 & 109 (12) \\
\hline thioridazine & chemical drug & & & & $6,70 E-03$ & DEPP1,HPN,SLCZA3 & \\
\hline vitamin K2 & chemical drug & & & & $6,70 \mathrm{E}-03$ & CD14,CSF1,SERPINB1 & \\
\hline magnesium & chemical- endogenous mammalian & & & & $6,70 E-03$ & BMPG,PTPAA2,RUNX2 & \\
\hline CxCL8 & cytokine & & 1,671 & bias & 6,73E-03 & CD44,FAS, ICAM1, IL18, КLKз,NFKB|A & 174 (17) \\
\hline TGFA & growth factor & & 1,371 & bias & 6,73E-03 & BIRC3,GBB2,GSTP1,ICAM1,LAMC2,SERPINE1 & $239(21)$ \\
\hline apigenin & hemical - endogenous non-mammalia & & $-1,513$ & bias & 6,73E-03 & 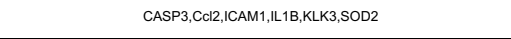 & $146(16)$ \\
\hline suz12 & enzyme & & 1,000 & & $6,90 E-03$ & IRAK3,KLF4,LAMB3,_LAMC2, LITAF,MGAT4B,Nes, PLCB4 & $79(5)$ \\
\hline L-glutanic acid & chemical-endogenous mammalian & Activated & 2,571 & bias & 6,93E-03 & CASP3,CCL2, GADD45B, IL 18,JUN,MGLL,RUNX2 & $202(20)$ \\
\hline Mapk & group & Activated & 2,000 & bias & 6,93E-03 & APOA1,CLDN4,L118,IRAK3,ITGB7,JUN,RUNX2 & $221(27)$ \\
\hline miR-1-3p (and other miRNAs w/sed GGAAUGU) & mature microRNA & Inhibited & $-3,123$ & bias & $6,94 \mathrm{E}-03$ & CSRP1,LASP1,LRP1,MOV10,NOTCH2,OAT,,PREX1,SDC4,SH2DAA,UNC93B1 & \\
\hline MAP2K4 & kinase & & 1,996 & bias & $6,96 E-03$ & BIRC3,CD44,CD86,FAS,JUN & $194(18)$ \\
\hline РTK2 & kinase & & $-0,371$ & bias & $6,96 E-03$ & BIRC3,CCI2,KRT8,PTK2B,SERPINB1 & $136(7)$ \\
\hline CCL11 & cyokine & Activated & 2,190 & bias & $6,96 E-03$ & BMPG,CXCL12,FAS, ICAM1,LL1B & $121(14)$ \\
\hline thioctic acid & chemical drug & & & & $6,96 E-03$ & CC12,1CAM1,JUN,NOO1,SOD2 & 139 (16) \\
\hline зм-001 & chemical drug & & 1,987 & bias & $7,01 E-03$ & CD40,СD83,СD86,L118 & $100(7)$ \\
\hline HSF2 & transcription regulator & & & & $7,01 E-03$ & Hspa 16,HSPB1,LL18,JUN & \\
\hline IL27RA & transmembrane receptor & & $-1,414$ & bias & $7,01 E-03$ & CD44,CD86,FAS, IL1B & $166(17)$ \\
\hline pentoxifylline & chemical drug & & $-1,091$ & bias & $7,01 E-03$ & CC12,ICAM1,IL1B,NFKBIA & $147(16)$ \\
\hline NR112 & ligand-dependent nuclear recepptor & & $-0,218$ & & $7,07 E-03$ & ALAS1,ANG, СEBPA,CYPBA7, GSTP1, Hamp/Hamp2, HMGCS2,JUN,PGD & $83(8)$ \\
\hline FFAR3 & G-protein coupled receptor & & 1,039 & & $7,14 E-03$ & BCL11A,CCR7,GBPG,ICAM1,L18,RCN1 & \\
\hline
\end{tabular}




\begin{tabular}{|c|c|c|c|c|c|c|c|c|}
\hline spironolactone & & chemical drug & Inhibited & $-2,418$ & bias & $7,14 \mathrm{E}-03$ & ВMP4,САSP3,СC12,СCR7,ІСAM1,L1B & $151(16)$ \\
\hline BCL2 & & transporter & & $-0,562$ & bias & $7,28 E-03$ & CASP3,FAS, GAP43, GZMA,ICAM1,LL18,NFKBIA & $178(18)$ \\
\hline miR-155-5p (miRNAs w/seed UAAUGCU) & & mature microRNA & Inhibited & $-2,945$ & bias & 7,35E-03 & 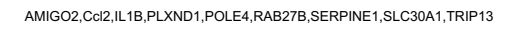 & $146(12)$ \\
\hline dalfampridine & & chemical drug & Activated & 2,191 & bias & $7,48 E-03$ & GADD45B, HK2,JUN,KLFA,SLCZA3 & $176(12)$ \\
\hline pravastatin & & chemical drug & & & & 7,48E-03 & CD40,ICAM1,L11, RHOU,SDC4 & $150(15)$ \\
\hline mettormin & & chemical drug & & $-0,500$ & bias & $7,64 \mathrm{E}-03$ & 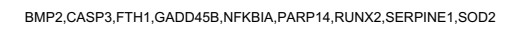 & $176(15)$ \\
\hline uranyl nitrate & & chemical toxicant & & 1,912 & & $7,64 \mathrm{E}-03$ & Cd24a, Clec2d (includes others), CSRP1,ESD,FBP1,FTL,RACK1 & \\
\hline TGFB2 & & growth factor & & $-0,116$ & bias & $7,64 E-03$ & BMP4,CD40, CD86,DOCK4,FAS,JAG1, SERPINE1 & 209 (19) \\
\hline CP-55940 & & chemical reagent & & & & $7,70 \mathrm{E}-03$ & CSF1,JUN,NFKBAA,TNFAPP3 & $86(7)$ \\
\hline DLL4 & & other & & 0,000 & & $7,70 \mathrm{E}-03$ & GATA3,MCM3,RELB,TCF7 & \\
\hline TWIST2 & & transcription regulator & & 0,000 & bias & $7,70 \mathrm{E}-03$ & BCL11A,CD44,L1 18,KLF6 & \\
\hline sumoз & & other & & 1,067 & & $7,70 \mathrm{E}-03$ & CAV2,CXCL16,HMGA1,RNASE4 & \\
\hline tempol & & chemical drug & & $-1,000$ & bias & $7,70 \mathrm{E}-03$ & BMP4,ICAM1,SERPINE1,SOD2 & $171(18)$ \\
\hline temozolomide & & chemical drug & & $-0,283$ & & $7,70 \mathrm{E}-03$ & CASP3,FAS,NFKBIA,SOD2 & $195(20)$ \\
\hline 11,12-epoxyeicosatrienoic acid & & chemical - endogenous mammalian & & & & $7,76 \mathrm{E}-03$ & CD44,ICAM1,NFKBIA & $100(9)$ \\
\hline ssRNA40 & & chemical reagent & & & & $7,76 \mathrm{E}-03$ & СD83,СО86, L1В & $144(16)$ \\
\hline wLs & & other & & & & $7,76 \mathrm{E}-03$ & BMP4,CC12,IL36G & \\
\hline MSR1 & & transmembrane receptor & & & & $7,76 E-03$ & ACOD1,CD40,1L1B & $138(15)$ \\
\hline GDF15 & 0,675 & growth factor & & & & $7,76 E-03$ & GDF 15,RND3,SERPINE1 & $153(9)$ \\
\hline TFAM & & transcription regulator & & & & $7,76 \mathrm{E}-03$ & FABP5, НК, „L1B & \\
\hline нохрз & & transcription regulator & & & & $7,76 \mathrm{E}-03$ & JUP,SDC1,SERPINE1 & \\
\hline CCND3 & & kinase & & & & $7,76 \mathrm{E}-03$ & СЕВРА,ККкз,МСМз & $114(7)$ \\
\hline M11RA & & transmembrane receptor & & & & $7,76 \mathrm{E}-03$ & $\mathrm{CCl} 2, \mathrm{CCl} 1, \mathrm{CLCA} 1$ & \\
\hline THZ1 & & chemical - kinase inhibitor & & & & $7,76 \mathrm{E}-03$ & BIRC3,CD2, IQSEC1 & \\
\hline sulfasalazine & & chemical drug & & & & $7,76 \mathrm{E}-03$ & ICAM1,LLBB,NFKBIA & $193(21)$ \\
\hline 2,4-dinitrofluorobenzene & & chemical toxicant & & & & $7,76 \mathrm{E}-03$ & CO12,ICAM1,L1B & $117(7)$ \\
\hline CD28 & 5,675 & transmembrane receptor & Activated & 2,339 & bias & $7,76 \mathrm{E}-03$ & 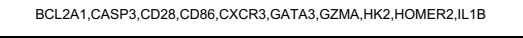 & $166(18)$ \\
\hline 3,4,5,3;4:-pentachlorobiphenyl & & chemical toxicant & & 1,461 & bias & $8,01 E-03$ & ВMP4,CD44,CYPBA7,GSTP1, JUN,NQO1 & $122(10)$ \\
\hline plicamycin & & chemical drug & & 0,114 & bias & $8,02 E-03$ & GJB2,ID1,Marcks,SERPINE1,SERPINE2,SLC11A2,WWC1 & $147(7)$ \\
\hline PTEES & & enzyme & & & & $8,03 E-03$ & CCI2,CXCL14,CYBA,LL18,SERPINE1 & $137(7)$ \\
\hline chloroquine & & chemical drug & & $-1,498$ & & $8,03 E-03$ & CD40,CD83, CD86, L1 18, RUNX2 & $190(20)$ \\
\hline cLock & & transcription regulator & & $-0,239$ & & $8,12 E-03$ & 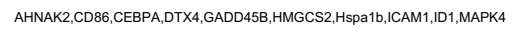 & \\
\hline E2F1 & & transcription regulator & & 1,269 & bias & $8,16 \mathrm{E}-03$ & 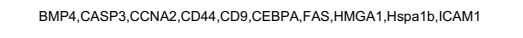 & $221(21)$ \\
\hline NR1H4 & & ligand-dependent nuclear receptor & & 0,233 & & $8,23 E-03$ & APOA 1, CCL2, CEBPA,FABP5, FAS, GSTA3,IL18,KLF4,SDC1 & $198(16)$ \\
\hline nicotinic acetylcholine receptor & & complex & & & & $8,40 \mathrm{E}-03$ & ID1,JUN & $137(7)$ \\
\hline triamcinolone hexacetonide & & chemical drug & & & & $8,40 \mathrm{E}-03$ & ICAM1,L1L1B & \\
\hline ANKRD42 & & transcription regulator & & & & $8,40 \mathrm{E}-03$ & CSF3,IL1B & \\
\hline
\end{tabular}




\begin{tabular}{|c|c|c|c|c|c|c|c|}
\hline SFRP5 & transmembrane receptor & & & & $8,40 E-03$ & $\mathrm{CO} 12,111 \mathrm{~B}$ & $96(7)$ \\
\hline valine & chemical-endogenous mammalian & & & & $8,40 E-03$ & $\mathrm{CD} 83, \mathrm{SOD} 2$ & \\
\hline iguratimod & chemical drug & & & & $8,40 E-03$ & $\mathrm{CCl} 2, \mathrm{CSF} 3$ & $144(14)$ \\
\hline Sik & group & & & & $8,40 E-03$ & ІСАМ 1 ,L1B & $196(24)$ \\
\hline HNRNPR & other & & & & $8,40 E-03$ & Cald1,Nes & \\
\hline TSC2201 & transcription regulator & & & & $8,40 E-03$ & IL1B,SERPINE1 & $103(10)$ \\
\hline $\mathrm{c}_{6}$ & other & & & & $8,40 E-03$ & $\mathrm{CO} 2,1 \mathrm{L11 \textrm {B }}$ & \\
\hline CHGA & other & & & & $8,40 E-03$ & IL1B,SERPINE2 2 & $146(7)$ \\
\hline ERBIN & other & & & & $8,40 E-03$ & Nrg1,SERPINE1 & \\
\hline KLKB1 & peptidase & & & & $8,40 E-03$ & ICAM1,KLF4 & \\
\hline TSPO & transmembrane receptor & & & & $8,40 E-03$ & ICAM1,SOD2 & \\
\hline teasaponin & hemical - endogenous non-mammaila & & & & $8,40 E-03$ & L1B,NFKBIA & \\
\hline WNT10B & other & & & & $8,40 E-03$ & CEBPA,RUNX2 & 130 (7) \\
\hline PLLRA & other & & & & $8,40 E-03$ & $\mathrm{CO} 12,111 \mathrm{~B}$ & $82(7)$ \\
\hline nicorandil & chemical drug & & & & $8,40 E-03$ & CASP3,JUN & $111(7)$ \\
\hline thenoyytrifluoroacetone & chemical reagent & & & & $8,40 E-03$ & BIRC3,SERPINE1 & \\
\hline AR-A014418 & chemical reagent & & & & $8,40 E-03$ & CD44,GDF15 & \\
\hline 6-methysulfirylhexy) isothicocyanate & chemical reagent & & & & $8,40 E-03$ & GSTP1,NQO1 & $45(3)$ \\
\hline zimelidine & chemical drug & & & & $8,40 E-03$ & DEPP1,HPN & \\
\hline $7 \mathrm{sk}$ & other & & & & $8,40 E-03$ & Cald1,Nes & \\
\hline manidipine & chemical drug & & & & $8,40 E-03$ & แ1B,JUN & $142(9)$ \\
\hline polyphosphate & chemical - endogenous mammalian & & & & $8,40 E-03$ & BMP2,ICAM1 & \\
\hline BAR501 & chemical reagent & & & & $8,40 E-03$ & $\mathrm{CO} 12,11 \mathrm{BB}$ & \\
\hline cyclosporin & biologic drug & & & & $8,40 E-03$ & ICAM1,LAMA2 2 & \\
\hline mir-30c-5p (and other miRNAs w/seed GUAAACA) & mature microRNA & Inhibited & $-2,580$ & bias & $8,41 E-03$ & CPNE8,JUN,PTGFRN,PTPRK,RAB278,RUNX2,SLC12A4 & \\
\hline BMPRAA & kinase & & 1,981 & bias & $8,45 \mathrm{E}-03$ & Hamp/Hamp2,ID1,ID3,SERPINE1 & $173(14)$ \\
\hline TFAP4 & transcription regulator & & $-0,762$ & & $8,45 E-03$ & CD44,CLDN4,GDF15,ID1 & \\
\hline NEDD9 & other & Activated & 2,000 & bias & $8,45 E-03$ & BMP2,GDF15,LRP1,SERPINE1 & \\
\hline propofol & chemical drug & & $-1,982$ & bias & $8,45 E-03$ & CASP3,СD14,L118,SOD2 & $175(13)$ \\
\hline IFNL1 & cyokine & Activated & 2,219 & bias & $8,47 E-03$ & CCR7,CD40,CD83,MLKL,OASL,SP110 & $172(17)$ \\
\hline FGF19 & growth factor & & & & $8,47 E-03$ & AMYZA,BTG3,FABP5,FBP1,ID1,Saa3 & \\
\hline ITGB1 & transmembrane receptor & & 1,175 & & $8,53 E-03$ & ADAM19, ICAM1,L1L18,ITGA9,ITGB7,JUN,SERPINE1, TNFAPP3 & $196(21)$ \\
\hline PKD1 & ion channel & & $-1,406$ & & $8,54 \mathrm{E}-03$ & 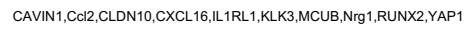 & $93(7)$ \\
\hline Smad2/3 & group & & & & $8,60 E-03$ & APOA1,GATA3,ID1,RUNX2,SERPINE1 & \\
\hline mitomycin C & chemical drug & Activated & 2,207 & bias & $8,60 \mathrm{E}-03$ & CASP3,FAS, TPRIPLL,JUN,NQO1 & $188(18)$ \\
\hline MED12 & transcription regulator & & & & $8,91 E-03$ & BMP4,GDF 15,POLE4 & \\
\hline ITGAM & transmembrane receptor & & & & $8,91 E-03$ & $\mathrm{CD} 28, \mathrm{CD} 86, \mathrm{IL} 1 \mathrm{~B}$ & 156 (19) \\
\hline
\end{tabular}




\begin{tabular}{|c|c|c|c|c|c|c|c|}
\hline ACKR1 & G-protein coupled receptor & & & & $8,91 E-03$ & ICAM1,IF1616,TNFA|P3 & \\
\hline socs6 & other & & & & $8,91 E-03$ & CEBPD,IL1B,Vnn3 & $113(10)$ \\
\hline MSX1 & transcription regulator & & & & $8,91 E-03$ & BMP2,BMP4,RUNX2 & \\
\hline NLRC5 & transcription regulator & & & & $8,91 E-03$ & CD40,HLA-A,IL1B & $142(17)$ \\
\hline 2-mercaptoacetate & chemical drug & & & & $8,91 E-03$ & CD68,ICAM1,IL1B & \\
\hline alendronic acid & chemical drug & & & & $8,91 E-03$ & ENPP1,ID 1,L1B & $91(7)$ \\
\hline nickel & chemical toxicant & & & & $8,91 E-03$ & CD86,ICAM1,SERPINE1 & $116(10)$ \\
\hline РІКзR1 & kinase & & 0,000 & bias & $8,95 E-03$ & CD44,CXCL12,ICAM1,L1818,SERPINE1,SOD2 & $171(16)$ \\
\hline MAFB & transcription regulator & & 0,277 & bias & $8,95 E-03$ & AKR1B10,ALCAM,CD28,CXCL 12,1L7R,OLFML2B & \\
\hline homocysteine & chemical - endogenous mammalian & & 1,370 & bias & $8,95 E-03$ & APOA1,CC12,CXCL12,ID1, IL18,Saa3 & $207(19)$ \\
\hline ZEB1 & transcription regulator & & & & $9,20 \mathrm{E}-03$ & GATA3,KRT19,LAMC2,SERPINE1,XDH & \\
\hline PLAUR & transmembrane receptor & & & & $9,24 \mathrm{E}-03$ & ANG,CC12,FOSL1,JUN & $188(18)$ \\
\hline ABL1 & kinase & & 1,921 & bias & $9,24 \mathrm{E}-03$ & BMP2,CCNA2,CSF1,FAS & $204(21)$ \\
\hline pimagedine & chemical drug & & & & $9,24 E-03$ & CASP3,GADD45B,ICAM1,SERPINE 1 & $86(7)$ \\
\hline tetraethylammonium & chemical drug & & $-1,982$ & bias & $9,24 E-03$ & EFHD2,FTH1,GST01,HSPB1 & \\
\hline prednisolone & chemical drug & & $-1,554$ & bias & $9,37 E-03$ & BIRC3,CASP3,CC12,CD86, CEBPD,FAS,GAB1,L118,SOD2,TNFAIP3 & $145(15)$ \\
\hline PRDM5 & transcription regulator & & & & $9,45 E-03$ & AРОВEC3B,CYP3A7,GADD45B,NOTCH2,RUN 2 , TINAGL1 & \\
\hline ERBB4 & kinase & & 0,447 & & 9,45E-03 & ADAM19,CXCL12,GAP43,JUN,RASA3,SERPINE2 2 & 191 (17) \\
\hline NUPR1 & transcription regulator & & 0,688 & bias & $9,57 E-03$ & 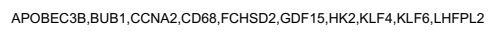 & \\
\hline PP1 & chemical -kinase inhibitor & & $-1,953$ & bias & 1,01E-02 & CXCL16,IL1B,JUN,SERPINE1 & \\
\hline HOTAIR & other & & 0.819 & & $1,011-02$ & GDF15,ICAM1,LAMB3,LAMC2 & \\
\hline LGR4 & transmembrane receptor & & $-1,091$ & bias & $1,011-02$ & CD14,HSD17B13,LL18,OAT & \\
\hline RCE1 & peptidase & & & & 1,01E-02 & 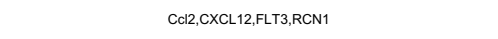 & \\
\hline SRC & kinase & Activated & 2,547 & bias & $1,01 E-02$ & CAVII1, CSF3,FOSL 1, ICAM1,ID1, JUN,KLKK3 & \\
\hline PARPBP & other & & & & 1,02E-02 & KRT8,TIAM1,TST & \\
\hline СамкII & complex & & & & 1,02E-02 & BIRC3,CD44,L118 & \\
\hline IKK (complex) & complex & & & & 1,02E-02 & BIRC3,ICAM1,L1B & \\
\hline SALL4 & transcription regulator & & & & 1,02E-02 & CD44,CEBPA,KRT19 & \\
\hline $\lg 61$ & complex & & & & 1,02E-02 & CD14,CD40,CD86 & \\
\hline ILRRA & transmembrane receptor & & & & 1,02E-02 & CD86,GZMA,JUN & \\
\hline miR-218-5p (and other miRNAs w/seed UGUGCUU) & mature microRNA & & & & 1,02E-02 & CASP3,LAMB3,RUNX2 & \\
\hline STK4 & kinase & & & & 1,02E-02 & FAS, ILRR,YAP1 & \\
\hline IGFBP5 & other & & & & 1,02E-02 & RUNX2,SERPINE1,SFN & \\
\hline PLA2G6 & enzyme & & & & $1,02 E-02$ & COl2,MRC1,RUNX2 & \\
\hline SFTPD & other & & & & 1,02E- -02 & CC12,CD14,L1B & \\
\hline GADD45A & other & & & & $1,02 E-02$ & IL1B,JUN,NEK2 & \\
\hline $\mathrm{CCN} 3$ & growth factor & & & & 1,02E-02 & BMP4,RUNX2,SERPINE1 & \\
\hline
\end{tabular}




\begin{tabular}{|c|c|c|c|c|c|c|c|c|}
\hline DYRK1A & & kinase & & & & $1,02 E-02$ & APOA 1, MCM3,SERPINE1 1 & \\
\hline ELF5 & & transcription regulator & & & & $1,02 E-02$ & GATA3,КLK3,КRT8 & \\
\hline Gm21596//mmgb1 & & transcription regulator & & & & $1,02 E-02$ & CXCL12,IL1B,ILIRL1 & \\
\hline probucol & & chemical drug & & & & $1,02 E-02$ & BMP2,CYBA, SERPINE1 & \\
\hline agmatine & & chemical- endogenous mammalian & & & & $1,02 E-02$ & ВMP2,BMP4,IСАM1 & \\
\hline fluticasone propionate & & chemical drug & & 0,000 & & $1,02 E-02$ & 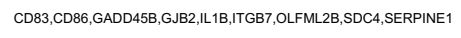 & \\
\hline WT1 & & transcription regulator & & 0,734 & & $1,03 E-02$ & BCL2A1,CSF 1,L18,JUP,KLF4,LRP1,PIR,SDC1,SERPINE1,SLC2A3 & \\
\hline MMP12 & & peptidase & & & & $1,05 E-02$ & CCIB, LLGST,NFKBIA,PLS3,VCL & \\
\hline gemcitabine & & chemical drug & & & & $1,05 E-02$ & CO12,CXCL12,FAS,FOSL1,L1B & \\
\hline 4-phenylbutyric acid & & chemical-endogenous mammalian & & $-0,816$ & & $1,05 E-02$ & CASP3, CCI2,CXCL12,HLA-A,PADI2,SOD2 & \\
\hline TO-901317 & & chemical reagent & & $-1,755$ & bias & $1,09 E-02$ & ALAS1,ALDH2,COL1,FABP5,FBP1, GSTP1,HK2,HMGCS2,LL18,MGLL & \\
\hline Laminin (complex) & & complex & & & & $1,10 \mathrm{E}-02$ & BIRC3,RUNX2,SDC4,SERPINE1 & \\
\hline sumo2 & & enzyme & & 1,067 & & $1,10 \mathrm{E}-02$ & CAV2,CXCL16, HMGA 1 ,RNASE4 & \\
\hline ATF1 & & transcription regulator & & & & 1,10E-02 & FOSL1,FTH1,UN,SOD2 2 & \\
\hline SAHM1 & & chemical reagent & Inhibited & $-2,000$ & bias & $1,10 \mathrm{E}-02$ & CD44,HK2,ID1,RHOU & \\
\hline UDP & & chemical-endogenous mammalian & & & & 1,111E-02 & COL1,SERPINE 1 & \\
\hline L-phenyylalanine & & chemical- endogenous mammalian & & & & $1,11 \mathrm{E}-02$ & FAS,SOD2 2 & \\
\hline saikosaponin A & & chemical reagent & & & & 1,11E-02 & BMP4,JUN & \\
\hline ganglioside GM3 & & chemical - endogenous mammalian & & & & $1,11 \mathrm{E}-02$ & CD40,ICAM1 & \\
\hline Calcineurin B & & group & & & & $1,111-02$ & B3GAT1,CXCR3 & \\
\hline Cypat14 & & enzyme & & & & $1,11 E-02$ & $\mathrm{CO} 2,1 \mathrm{L11 \textrm {B }}$ & \\
\hline ibudilast & & chemical drug & & & & $1,11 \mathrm{E}-02$ & "L1B,NCS1 & \\
\hline tectorigenin & & hemical - endogenous non-mammalia & & & & $1,11 \mathrm{E}-02$ & ICAM1, LL1B & \\
\hline GPR84 & & G-protein coupled receptor & & & & $1,11 E-02$ & $\mathrm{CO} 12,111 \mathrm{~B}$ & \\
\hline GSTK1 & & enzyme & & & & $1,111-02$ & COL2,TMEM173 & \\
\hline ADGRF5 & & G-protein coupled receptor & & & & $1,11 E-02$ & $\mathrm{CCl} 2, \mathrm{CSF} 1$ & \\
\hline LTBP4 & & growth factor & & & & $1,11 E-02$ & BмP4,ID1 & \\
\hline SMAD $1 / 5$ & & group & & & & $1,111-02$ & 101,103 & \\
\hline ॥26 & & cytokine & & & & $1,111-02$ & ІСАM 1 ,L1B & \\
\hline $\mathrm{ABCC} 1$ & & transporter & & & & $1,11 E-02$ & CYBA,NQO1 & \\
\hline PDLIM7 & & other & & & & $1,111-02$ & BMP2,RUNX2 & \\
\hline CD8A & 8,241 & other & & & & $1,11 E-02$ & CASP, IL1B & \\
\hline SLC25A4 & & transporter & & & & $1,11 \mathrm{E}-02$ & BIRC3,SOD2 & \\
\hline МАРЗК12 & & kinase & & & & 1,11E-02 & CASP3,JUN & \\
\hline CAV3 & & enzyme & & & & $1,11 E-02$ & CAV2,DMD & \\
\hline mir-326 & & microRNA & & & & $1,11 E-02$ & NOTCH2,SMO & \\
\hline levetiractam & & chemical drug & & & & $1,11 E-02$ & GAP43,L1B & \\
\hline
\end{tabular}




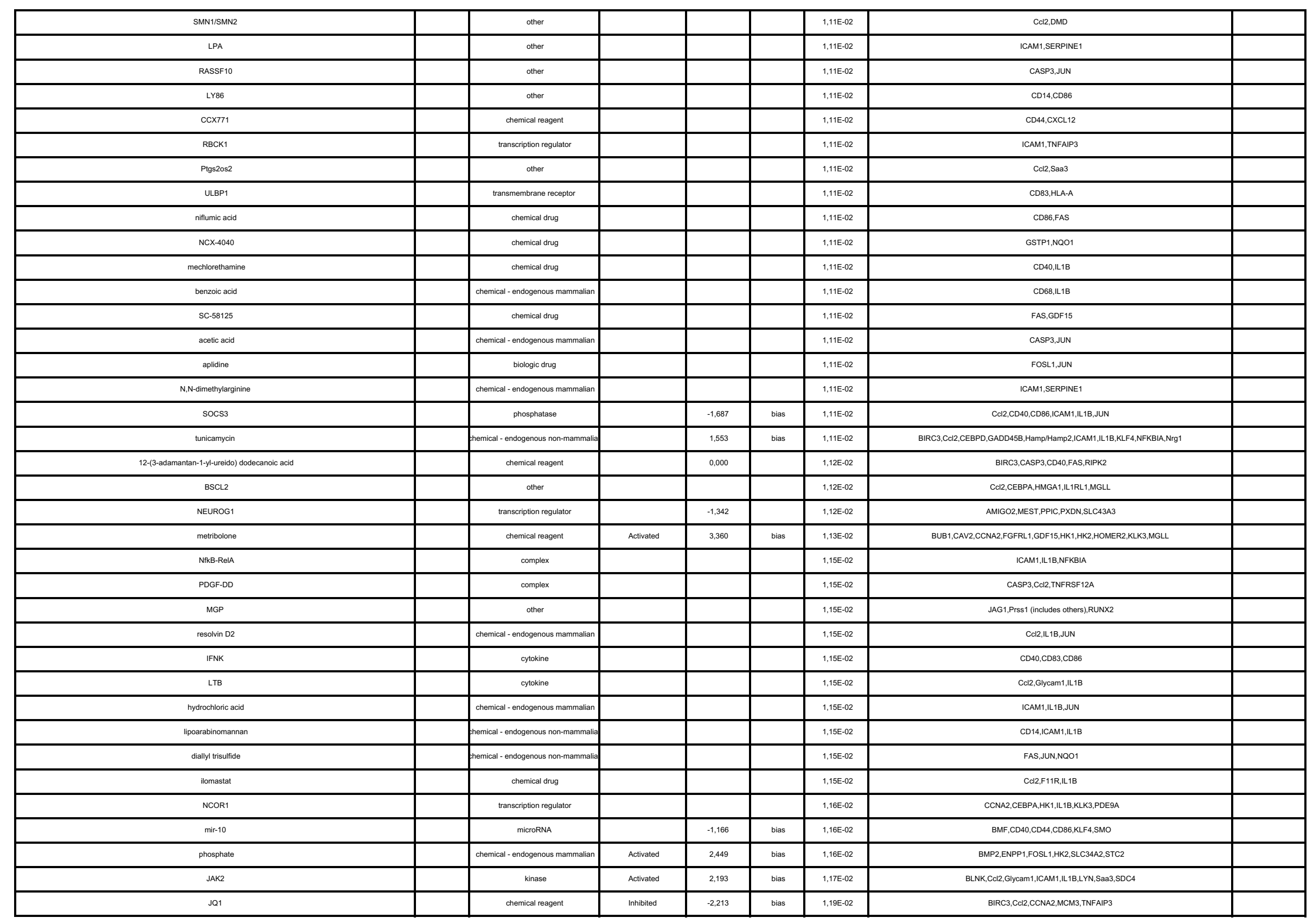




\begin{tabular}{|c|c|c|c|c|c|c|c|c|}
\hline ACVR1 & & kinase & & & & 1,19E-02 & C1R,Hamp/Hamp2,ICAM1,ID1 & \\
\hline mir-200b-3p (and other mirNAs wsseed AAUACUG) & & mature microRNA & & $-1,944$ & bias & 1,19E-02 & BST1,JAG1,PERP,PTPN13 & \\
\hline EED & & transcription regulator & & & & 1,19E-02 & GATA3,KLF4,RUNX2,SCIN & \\
\hline SELPLG & & other & Activated & 2,000 & bias & 1,19E-02 & BCL2A1,BST1,LL1B,LAG3 & \\
\hline CALR & & transcription regulator & & & & 1,19E-02 & COL2,CEBPA,CAMI,VCL & \\
\hline GABPA & & transcription regulator & & & & 1,19E-02 & FAS, ILRR,NQ01, RRAS & \\
\hline 4-hydroxynonenal & & chemical toxicant & & 1,091 & bias & 1,19E-02 & ICAM1,L18,JUN,SERPINE1 & \\
\hline $\mathrm{N}-[\mathrm{N}-3,3,-$-difluorophenacety-L-Ala)]-S-phenylyycine t-buty lester & & chemical - protease inhibitior & Inhibited & $-2,000$ & bias & 1,19E-02 & CSF1,L1B,RELB,RND3 & \\
\hline NCOA 1 & & transcription regulator & & 1,331 & bias & $1,22 \mathrm{E}-02$ & CEBPA,CHD9, GJB2, KLLK3,MUC1,NFKBBA,NFKBBZ,SERPINE1 & \\
\hline $\operatorname{CDX} 2$ & & transcription regulator & & 1,032 & bias & $1,22 \mathrm{E}-02$ & CD9, JUN,KLF4,KRT 23,LAMC2,Muc1,SCIN,UGT1A7 (includes others) & \\
\hline E2F3 & & transcription regulator & & 0,478 & bias & $1,22 \mathrm{E}-02$ & 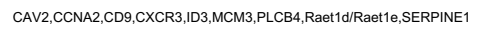 & \\
\hline HOXА⿱ & & transcription regulator & & 1,949 & bias & $1,22 E-02$ & 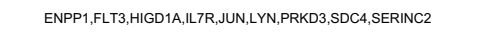 & \\
\hline FGF8 & & growth factor & & 0,747 & bias & $1,22 \mathrm{E}-02$ & BMP2,BMP4,СSF3,LAMB3,RUNх2,TRIP6 & \\
\hline CIP2A & & other & & $-0,600$ & bias & $1,26 \mathrm{E}-02$ & HK2,LASP1,S100A16,SCIN,SERPINE2 & \\
\hline miR-29b-3p (and other miRNAs w/seed AGCACCA) & & mature microRNA & Inhibited & $-2,182$ & bias & $1,26 \mathrm{E}-02$ & CAV2,KLF4,PPIC,TNFAlP3,TPm1 & \\
\hline TBP & & transcription regulator & & & & $1,26 \mathrm{E}-02$ & BCL2A1,HLA-A,NFKBAA,TNFAPB,XDH & \\
\hline Wnt & & group & & & & $1,29 E-02$ & CD44,CEBPA,GATA3,RUN $\times 2$ & \\
\hline АLB & & transporter & & 1,982 & bias & 1,292E-02 & САРP3, СС12,СУBA,L1L1B & \\
\hline RAC2 & & enzyme & & & & 1,29E-02 & Cd24a,LL1, RUNX2,ZYXX & \\
\hline TNC & & other & & & & $1,29 \mathrm{E}-02$ & COL12,CD8A,CXCL12,111B & \\
\hline OGT & & enzyme & & $-0,928$ & & 1,29E-02 & DTNA,GAP43,HMGA1,JUN,JUP,NEK2 & \\
\hline leukotriene B4 & & chemical - endogenous mammalian & & & & $1,30 \mathrm{E}-02$ & CC12,L118,JUN & \\
\hline dexamethasone phosphate & & chemical drug & & & & $1,30 \mathrm{E}-02$ & ID3,|LLB,NFKB|A & \\
\hline deoxycorticosterone acetate & & chemical drug & & & & $1,30=-02$ & Cald1,CD68,CYBA & \\
\hline Ciap & & group & & & & $1,30 \mathrm{E}-02$ & CCl2,CSF3,LL1B & \\
\hline daporinad & & chemical drug & & & & $1,30 \mathrm{E}-02$ & ID1,SERPINE1,TRIP13 & \\
\hline GRHL2 & & transcription regulator & & & & $1,30 \mathrm{E}-02$ & BMP2,CLDN4,SEMA3B & \\
\hline CD81 & 0,944 & other & & & & $1,30 \mathrm{E}-02$ & CD86,ICAM1,ID1 & \\
\hline ॥36G & 6,681 & cytokine & & & & $1,30 \mathrm{E}-02$ & CD83,СSF3,MRC1 & \\
\hline Cokn1c & & other & & & & $1,30 \mathrm{E}-02$ & CCNA2,MAL,MCM3 & \\
\hline AZGP1 & & transporter & & & & 1,30E-02 & ВMP4,HK2,KRT19 & \\
\hline carvedilol & & chemical drug & & & & 1,30E-02 & СYBA,GAР43,L1L1B & \\
\hline $\mathrm{N}^{\mathrm{N} 2+}$ & & chemical reagent & & & & $1,30 \mathrm{E}-02$ & BIRC3,CSF1,ICAM1 & \\
\hline RUNX2 & 2,637 & transcripition regulator & & 1,726 & bias & $1,31 E-02$ & BMP2,CEBPA,CEBPD,GADD 45B,GALNT3,RUNX2,SERPINE1 & \\
\hline TEAD1 & & transcription regulator & Activated & 2,236 & bias & $1,34 E-02$ & ARIDSB,FJX1,LATS2,NFKBZZ,TRNP1 1 & \\
\hline F3 & & transmembrane receptor & & 1,951 & bias & $1,34 \mathrm{E}-02$ & CCI2,DOCK4,ICAM1,LL18,SERPINE1 & \\
\hline uric acid & & chenical - endogenous mammalian & & 1,178 & & $1,35 \mathrm{E}-02$ & 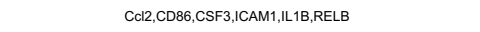 & \\
\hline
\end{tabular}




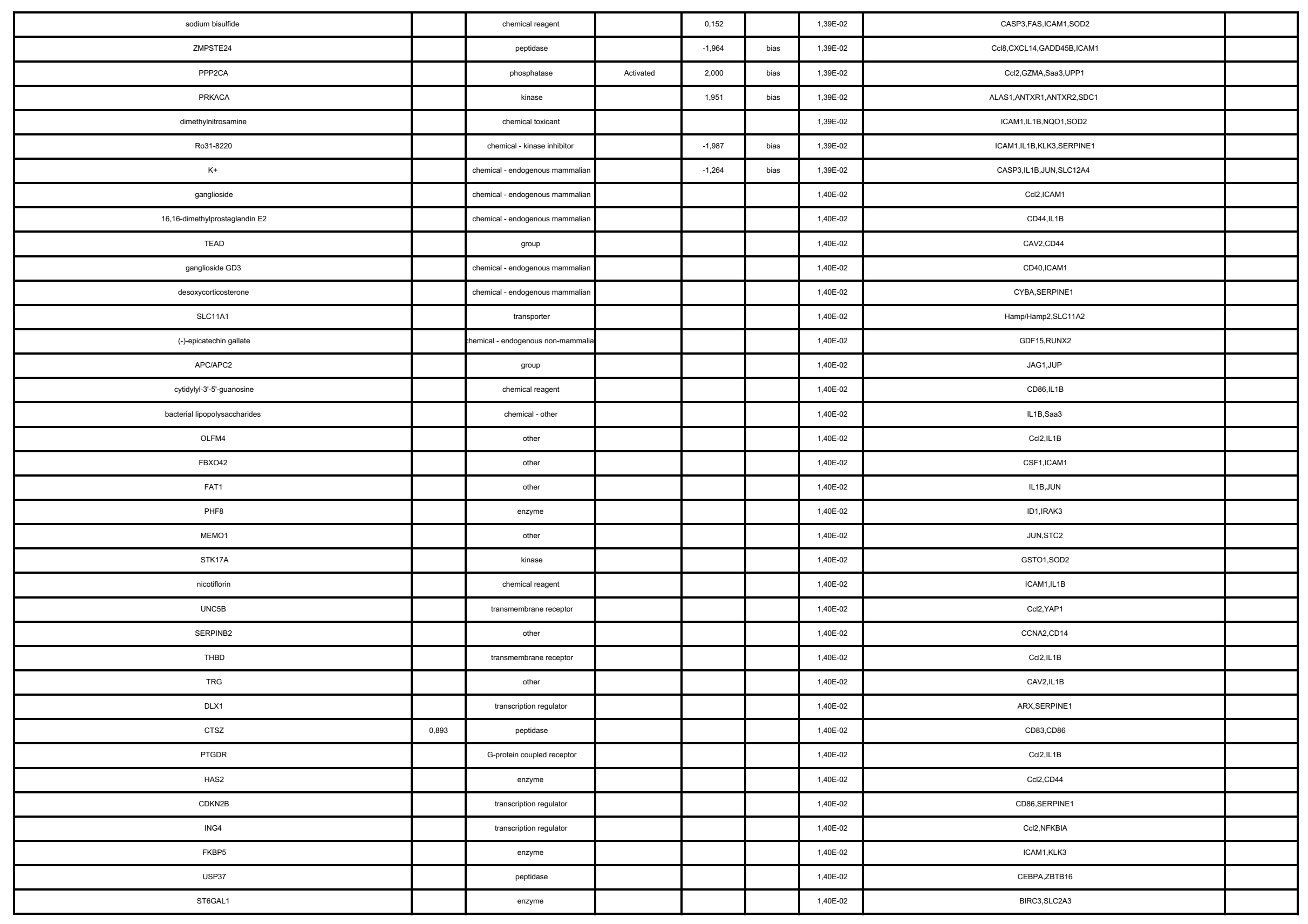




\begin{tabular}{|c|c|c|c|c|c|c|c|c|}
\hline сосн & & other & & & & $1,40 \mathrm{E}-02$ & $\mathrm{CO12,111 \textrm {B }}$ & \\
\hline IL22RA1 & & transmembrane receptor & & & & 1,40E-02 & Hamp/Hamp2,SOD2 & \\
\hline cyclic guanosine monophosphate-adenosine monophosphate & & hremical - endogenous non-mammalia & & & & $1,40 \mathrm{E}-02$ & CD86,IL1B & \\
\hline asbestos & & chemical toxicant & & & & $1,40 \mathrm{E}-02$ & FOSLL,JUN & \\
\hline 6-aminonicotinamide & & chemical reagent & & & & $1,40 \mathrm{E}-02$ & $\mathrm{Ccl}_{2}, \mathrm{Saa3}$ & \\
\hline sulindac sulfone & & chemical-endogenous mammalian & & & & $1,40 \mathrm{E}-02$ & NQO1,PRKG1 & \\
\hline bupivacaine & & chemical drug & & & & $1,40 \mathrm{E}-02$ & FOSL1,HPGDS & \\
\hline ketorolac & & chemical drug & & & & 1,40E-02 & COLI,NFKBIA & \\
\hline SR1078 & & chemical reagent & & & & $1,40 \mathrm{E}-02$ & CO12,PADI2 & \\
\hline miR-182 inhibitor & & chemical reagent & & & & $1,40 \mathrm{E}-02$ & CASP3,NFKBIA & \\
\hline 2,4-dinitrobenzzenesuffonic acid & & chemical reagent & & & & $1,40 \mathrm{E}-02$ & CD86,ICAM1 & \\
\hline YBX1 & & transcription regulator & & 1,982 & bias & 1,42E-02 & COL1,111,1RF5, LLF4,SLC2A3,STC2 & \\
\hline ІТСвз & & transmembrane receptor & & 0,200 & bias & 1,42E-02 & CD40,CSF1,LL1B,RUNX2,SERPINE1 & \\
\hline CHD7 & & enzyme & & & & 1,45E-02 & JAG1,PERP,POLE4 & \\
\hline Smad & & complex & & & & 1,45E-02 & ID1,ID3,SERPINE1 & \\
\hline BCL2L11 & & other & & & & 1,45E-02 & CASP3, СC21,L11B & \\
\hline mir-124 & & microRNA & & & & 1,45E-02 & CASP3,CC12,RUNX2 & \\
\hline BTG2 & & transcription regulator & & & & 1,45E-02 & "L18,NQ001,S002 & \\
\hline BAG1 & & other & & & & $1,45 E-02$ & Hspa 16,JUN,KLK3 & \\
\hline hemocyanin & & biologic drug & & & & 1,45E-02 & CD14,CD83,CD86 & \\
\hline amitriptyline & & chemical drug & & & & 1,45E-02 & DEPP1,HPN,SLC2A3 & \\
\hline piceatannol & & hremical - endogenous non-mammalia & & & & 1,45E-02 & FAS,ICAM1, L1 1B & \\
\hline kaempferol & & chemical toxicant & & & & 1,45E-02 & ICAM1,JUN,RUNX2 & \\
\hline roscovitine & & chemical drug & & $-1,954$ & bias & $1,50 \mathrm{E}-02$ & CD44,CEBPA,Raet1d/Raet1e,RALA & \\
\hline EGR3 & & transcription regulator & & 1,980 & & $1,50 \mathrm{E}-02$ & ESD,ID3,JUN,PLXND 1 & \\
\hline TEAD3 & & transcription regulator & Activated & 2,000 & bias & $1,50 \mathrm{E}-02$ & ARIDSB,FJX1,NFKBIZ,TRNP1 & \\
\hline ABCA1 & & transporter & & $-1,009$ & & $1,50 \mathrm{E}-02$ & APOA 1, CC12,CSF3,L11B & \\
\hline N-acetyssphingosine & & chemical reagent & & 1,969 & bias & $1,50 \mathrm{E}-02$ & CC12,JUN,SERPINE1,SOD2 & \\
\hline clopidogrel & & chemical drug & & $-1,342$ & & $1,50 \mathrm{E}-02$ & АТОH8,ВMF,CXCL12,DHRS3,STC2 & \\
\hline thyroid hormone & & chemical-endogenous mammalian & & $-1,342$ & & 1,53E-02 & CAMK4,CCNA2,CD2,ID1,OAT,PGD,RTN1,SERPINE2,TCEA3 & \\
\hline DMD & 1,365 & other & & & & 1,55E-02 & BLNK,DMDO,FTH1,Marcks, PADI2,PTGFRN,SCIN,SERPINB 1,SYNC,WWC1 & \\
\hline PC-SPES & & chemical drug & & & & 1,56E-02 & CCNA2, CD9, FTL,D1, L1 18,KLK3 & \\
\hline LCK & 1,108 & kinase & & & & 1,59E-02 & CCNA2,CD2,GADD45B,JUN,NOTCH2 & \\
\hline ID1 & 1,349 & transcription regulator & & 1,360 & bias & 1,59E-02 & DTX4,GATAB,ICAM1,F16,RUNX2 & \\
\hline sulindac surfide & & chemical drug & & 1,460 & & 1,59E-02 & ESD, GDF 15,KRT19,NQ01,STC2 & \\
\hline ADRA1D & & G-protein coupled receptor & & & & $1,61 \mathrm{E}-02$ & CEBPD,FABP5,ILGST,JUN & \\
\hline herbimycin & & chemical - kinase inhibitor & & $-1,118$ & bias & 1,61E-02 & ICAM1,L1B,SERPINE1,SOD2 & \\
\hline
\end{tabular}




\begin{tabular}{|c|c|c|c|c|c|c|c|}
\hline Sb202190 & & chemical - kinase inhibitor & & $-1,980$ & bias & $1,61 E-02$ & CCI2,CD86,ICAM1, LL18,MAPK6,SERPINE1,XDH \\
\hline Trp53cor1 & & other & & & & $1,61 E-02$ & CCl2,IL1B,PERP \\
\hline cxCL10 & & cytokine & & & & $1,61 \mathrm{E}-02$ & COI2,CXCR3,L118 \\
\hline LTBP1 & & other & & & & $1,61 \mathrm{E}-02$ & BMF,GATA3,SERPINE1 \\
\hline FGF9 & & growth factor & & & & $1,61 E-02$ & BMP4,CSF3,RUNX2 \\
\hline HMGB2 & & transcription regulator & & & & $1,61 E-02$ & CSF3,LLB, RUN X2 \\
\hline k-252 & & chemical-kinase inhibitor & & & & $1,61 \mathrm{E}-02$ & CD14,JUN,SOD2 \\
\hline geranylgeranyl pyrophosphate & & chemical-endogenous manmmalian & & & & $1,61 E-02$ & ВMP2,ICAM1,IL1B \\
\hline linolenic acid & & chemical- endogenous mammalian & & & & $1,61 \mathrm{E}-02$ & ICAM1,NFKBIA,SERPINE1 \\
\hline СEBPD & 0,429 & transcription regulator & & 0,447 & bias & $1,63 E-02$ & BCL2A1,CC12,CD14,CEBPD, IL 18, Saаa \\
\hline linoleic acid & & chemical-endogenous manmmalian & & 0,468 & bias & $1,68 E-02$ & APOA 1, CCI2, ICAMM, LL IB, NFKBBA \\
\hline АТM & & kinase & & & & $1,68 \mathrm{E}-02$ & FAS,GADD45B,JUN,SERPINE1,TNFAPP3 \\
\hline ETS2 & & transcription regulator & Activated & 2,236 & bias & $1,68 \mathrm{E}-02$ & 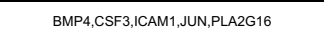 \\
\hline 11-6-hydroxymethy-chiro-inosito 2-(R)-2-O-methy-3-O-Octadecylcarbonate & & chemical - kinase inhibitor & & & & $1,73 E-02$ & CXCL16,GDF15 \\
\hline тнос5 & & other & & & & $1,73 E-02$ & APOA 1, CEBPA \\
\hline DPY30 & & other & & & & $1,73 E-02$ & 101,103 \\
\hline ADCY7 & & enzyme & & & & $1,73 E-02$ & ILRL1,RAK2 \\
\hline FOXN1 1 & & transcription regulator & & & & $1,73 E-02$ & GZMA,SERPINB1 \\
\hline SEMAAD & & transmembrane receptor & & & & $1,73 E-02$ & CD40,RRAS \\
\hline ITGA4 & & transmembrane receptor & & & & $1,73 E-02$ & L1B,TNFAPB \\
\hline AMBP & & transporter & & & & $1,73 E-02$ & $\mathrm{CO} 21,111 \mathrm{~B}$ \\
\hline SPRY4-IT1 & & other & & & & $1,73 E-02$ & F11R,ZNF703 \\
\hline FRMDG & & other & & & & $1,73 E-02$ & BMP2,RASSF 4 \\
\hline HLA-G & & other & & & & $1,73 E-02$ & CDBA,FAS \\
\hline LUM & & other & & & & $1,73 E-02$ & FAS,IL1B \\
\hline LY9 & 2,405 & other & & & & $1,73 E-02$ & CD81,ZBTB16 \\
\hline Igkv1-117 & & other & & & & $1,73 \mathrm{E}-02$ & JUN,RELB \\
\hline Sry & & transcription regulator & & & & $1,73 E-02$ & แL1B,SOD2 \\
\hline NFKBIE & & transcription regulator & & & & $1,73 E-02$ & CSF3,ICAM1 \\
\hline PA264 & & transcription regulator & & & & $1,73 E-02$ & GDF15,KLK3 \\
\hline нвв & & transporter & & & & $1,73 E-02$ & ICAM1,SERPINE1 \\
\hline COP1 & & enzyme & & & & $1,73 E-02$ & FAS,ICAM1 \\
\hline S100P & & other & & & & $1,73 E-02$ & KRT19,KRT8 \\
\hline СYBA & 1,652 & enzyme & & & & $1,73 E-02$ & SERPINE1,SOD2 \\
\hline zilieuton & & chemical drug & & & & $1,73 E-02$ & Col1,ICAM1 \\
\hline astressin $2 B$ & & biologic drug & & & & $1,73 E-02$ & CO12,SLC2A3 \\
\hline polymyxin B & & biologic drug & & & & $1,73 E-02$ & АРОВЕСЗВ,LL1B \\
\hline
\end{tabular}




\begin{tabular}{|c|c|c|c|c|c|c|c|}
\hline S-equal & chemical drug & & & & $1,73 \mathrm{E}-02$ & CD86,SERPINE1 & \\
\hline formononetin & hemical - endogenous non-mammalia & & & & $1,73 \mathrm{E}-02$ & BMP2,СD86 & \\
\hline Iysophosphatidylinositol & chemical- endogenous mammalian & Activated & 2,000 & bias & 1,73E- -02 & 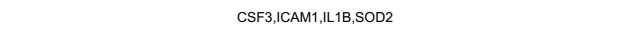 & \\
\hline ЈАКз & kinase & & & & $1,73 \mathrm{E}-02$ & CSF3,GATA3,LIRR,LAG3 & \\
\hline E2F2 & transcription regulator & & & & $1,78 E-02$ & CCNA2,ID1,MCM3,Raet1d/Raet1e,SERPINE1 & \\
\hline PSEN1 & peptidase & & $-0,762$ & & $1,78 E-02$ & AMYZA,BASP1,C1R,CASP3,CD9, CTSZ,GADD45B,GSTP1,HK1,L1 18 & \\
\hline glutamine & chemical-endogenous mammalian & Inhibited & $-2,000$ & bias & 1,79E-02 & FABP5,HK1, ICAM1,JUN,KLF4,NFKBIA & \\
\hline tetrodotoxin & chemical drug & & & & $1,79 \mathrm{E}-02$ & AMIGO2,FAS,D1 & \\
\hline PRKDC & kinase & & & & $1,79 E-02$ & BIRC3,КLKК,PREX1 & \\
\hline ctss & peptidase & & & & $1,79 E-02$ & CASP3,FAS,IL1B & \\
\hline mir-23 & microRNA & & & & $1,79 E-02$ & PTK2B,RUNX2,SOD2 & \\
\hline ICOSLGLOC102723996 & other & & & & 1,79E-02 & CD28,IL1B,IL1RL1 & \\
\hline PTPN22 & phosphatase & & & & $1,79 E-02$ & IL1B,LAG3,MRC1 & \\
\hline ESRRB & $\begin{array}{l}\text { ligand-dependent nuclear r receptor } \\
\end{array}$ & & & & 1,79E-02 & HK2,Hspa 1b,POLE4 & \\
\hline ozone & chemical toxicant & & & & 1,79E-02 & COC1,L118,JUN & \\
\hline PML & transcription regulator & & $-1,066$ & & $1,81 \mathrm{E}-02$ & APOA1,FAS, FTL,HSPB 1, L1 18,JUP,NQ01, TNFAPP3 & \\
\hline isoproterenol & chemical drug & & 1,860 & bias & $1,81 \mathrm{E}-02$ & ALDH3B1,CSF3,DHRS3,FOSL1,LL18,JUN,PRKD3,SOD2 & \\
\hline FGFR2 & kinase & & & & $1,81 \mathrm{E}-02$ & BMP4,FAS,ICAM1,L1B, & \\
\hline EPAS1 & transcription regulator & Activated & 2,404 & bias & $1,84 E-02$ & BIRC3, CEBPA,GADD458,SERPINE1,SLC11A2,SLC2A3,SOO2,STC2,TNFAIP3,YAP1 & \\
\hline SCARB1 & transporter & & $-1,387$ & & 1,85E-02 & APOA1,CD68,ICAM1,LL1B & \\
\hline ITGA5 & transmembrane receptor & & & & 1,85E-02 & CD40,CD44,LL18,JUN & \\
\hline RETN & other & & 1,067 & bias & $1,85 E-02$ & APOA 1, FABPF, ICAMM, SERPINE1 & \\
\hline TEAD2 & transcription regulator & Activated & 2,000 & bias & $1,85 E-02$ & ARIDSB,FJX1,NFKBIZ,TRNP1 & \\
\hline ATF4 & transcription regulator & & & & 1,93E-02 & GDF15,JAG1,JUN,KLF4,SOD2,STC2,TNFRSF12A,XDH & \\
\hline STAT4 & transcription regulator & Activated & 2,800 & bias & 1,94E- -02 & GATA3,Hspa 16,LRP1,Ms4aAb (includes others),RCN3,SERPINB 1,SERPINE1,SLC2A3,STC2,Tef7 & \\
\hline N-cor & group & & & & 1,95E-02 & CC18,CXCL14,DHRS3, GSTP1, 1L18,ZNFFO3 & \\
\hline Mir122a,b & group & & & & 1,97E-02 & CO12,CD68, IL1B & \\
\hline CDH11 & other & & & & 1,97E-02 & CD9,JUP,PTK2B & \\
\hline USP7 & peptidase & & & & 1,976-02 & BMF,КLКз,РDE9А & \\
\hline PLA2R1 & transmembrane receptor & & & & 1,97E-02 & ALDH2,BDH2,SOD2 2 & \\
\hline FABP4 & transporter & & & & 1,97E-02 & CCl2,FABP5,LL1B & \\
\hline CD47 & transmembrane receptor & & & & 1,97E-02 & CSF1,FTL,LL1B & \\
\hline imipramine & chemical drug & & & & $1,97 \mathrm{E}-02$ & DEPP1,HPN,SLC2A3 & \\
\hline BTNL2 & transmembrane receptor & & 1,342 & bias & $1,97 E-02$ & GJB2,Hspa16,IL1RL1,SPECC1,XDH & \\
\hline triadimefon & chemical toxicant & & 1,000 & bias & $1,98 E-02$ & ALAS1,CYPBA7,GSTA3,TST & \\
\hline fish oils & chemical drug & & & & $1,98 E-02$ & APOA1,CII2,CEBPA,IL1B & \\
\hline SQSTM1 & transcription regulator & & & & 1,986-02 & «LB, JUN,NFKBA, NQO1 & \\
\hline
\end{tabular}




\begin{tabular}{|c|c|c|c|c|c|}
\hline culs & enzyme & $-0,849$ & $1,98 E-02$ & NQO1,PLS3,Prss 1 (includes others), TCEA3 & \\
\hline ADRA1A & G-protein coupled receptor & & $1,98 E-02$ & CEBPD,FABP5,IL6ST,JUN & \\
\hline Fox03 & transcription regulator & 1,597 & $2,04 E-02$ & ANXA3,BMP2,FABP5, GADD45B, HMGCS2,ICAM1,JAG1,LCP2,NFKB|A,NTN4 & \\
\hline 9-hydroxy-(S)-10,12-octadecadienoic acid & chemical-endogenous mammalian & & $2,07 E-02$ & SERPINE1 & \\
\hline acetoacetyl-coenzyme A & chemical-endogenous mammalian & & $2,07 E-02$ & М1в & \\
\hline endocannabinoid & chemical - endogenous mammalian & & $2,07 E-02$ & APOA1 & \\
\hline GPSM3 & other & & $2,07 E-02$ & แ1в & \\
\hline сомм05 & other & & $2,07 E-02$ & NOTCH2 & \\
\hline rasburicase & biologic drug & & $2,07 E-02$ & $\mathrm{Ccl} 2$ & \\
\hline BDNF-AS & other & & $2,07 E-02$ & CASP3 & \\
\hline вмР2/4 & group & & 2,07E-02 & Hampl/tamp2 & \\
\hline Patched & group & & $2,07 E-02$ & sмо & \\
\hline IRAK1/4 & group & & $2,07 E-02$ & М1в & \\
\hline glutethimide & chemical drug & & 2,07E-02 & ALAS1 & \\
\hline G-Actin & group & & $2,07 E-02$ & Jun & \\
\hline acridine half-mustard & chemical toxicant & & $2,07 E-02$ & ANTXR2 & \\
\hline wsx1-gp 130 & complex & & $2,07 E-02$ & GATA3 & \\
\hline bovine testicular hyaluronidase & biologic drug & & $2,07 E-02$ & CD44 & \\
\hline РMM2 & enzyme & & $2,07 E-02$ & ICAM1 & \\
\hline NLRP2 & other & & $2,07 E-02$ & ICAM1 & \\
\hline$\| 1$ bos & other & & $2,07 E-02$ & แ1в & \\
\hline PELI3 & enzyme & & $2,07 E-02$ & М1в & \\
\hline WFDC1 & other & & $2,07 E-02$ & ICAM1 & \\
\hline СМРК2 & kinase & & $2,07 E-02$ & М1в & \\
\hline PPIP5K1 & phosphatase & & $2,07 E-02$ & RUNX2 & \\
\hline SV2B & transporter & & $2,07 E-02$ & SYT1 & \\
\hline SNORD21 & other & & $2,07 E-02$ & HLA-A & \\
\hline 1002 & enzyme & & $2,07 E-02$ & CD40 & \\
\hline FNDC3B & other & & $2,07 E-02$ & RUNX2 & \\
\hline CLPX & enzyme & & $2,07 E-02$ & ALAS1 & \\
\hline DAGLA & enzyme & & $2,07 E-02$ & М1в & \\
\hline UBN1 & transcripition regulator & & $2,07 E-02$ & CCNA2 & \\
\hline canakinumab & biologic drug & & $2,07 E-02$ & М1в & \\
\hline DEPDC1 & transcription regulator & & $2,07 E-02$ & TNFAPB & \\
\hline SGMS2 & enzyme & & $2,07 E-02$ & APOA 1 & \\
\hline MYADM & other & & $2,07 E-02$ & ICAM1 & \\
\hline MACROD1 & enzyme & & $2,07 E-02$ & NFKBAA & \\
\hline
\end{tabular}




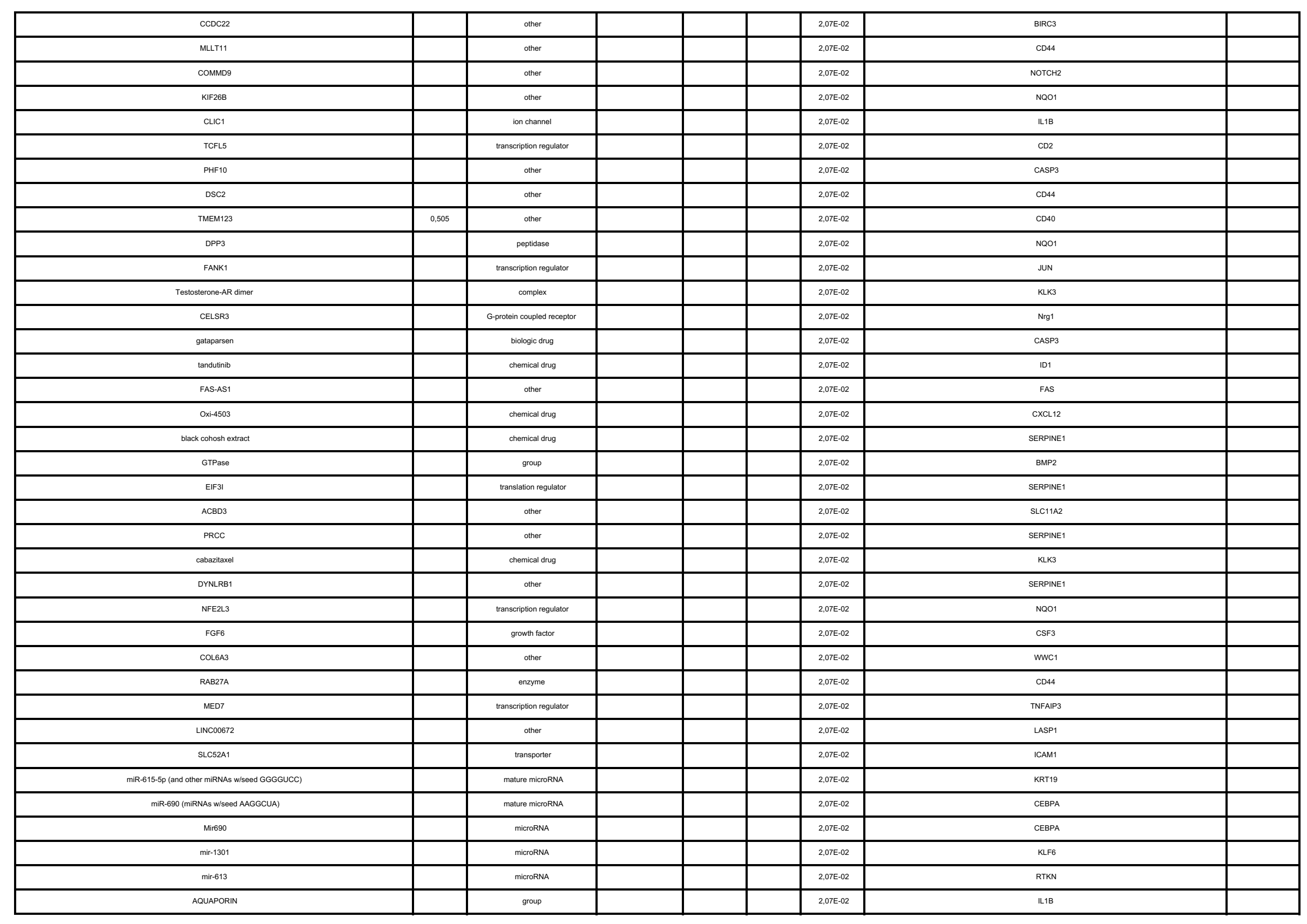




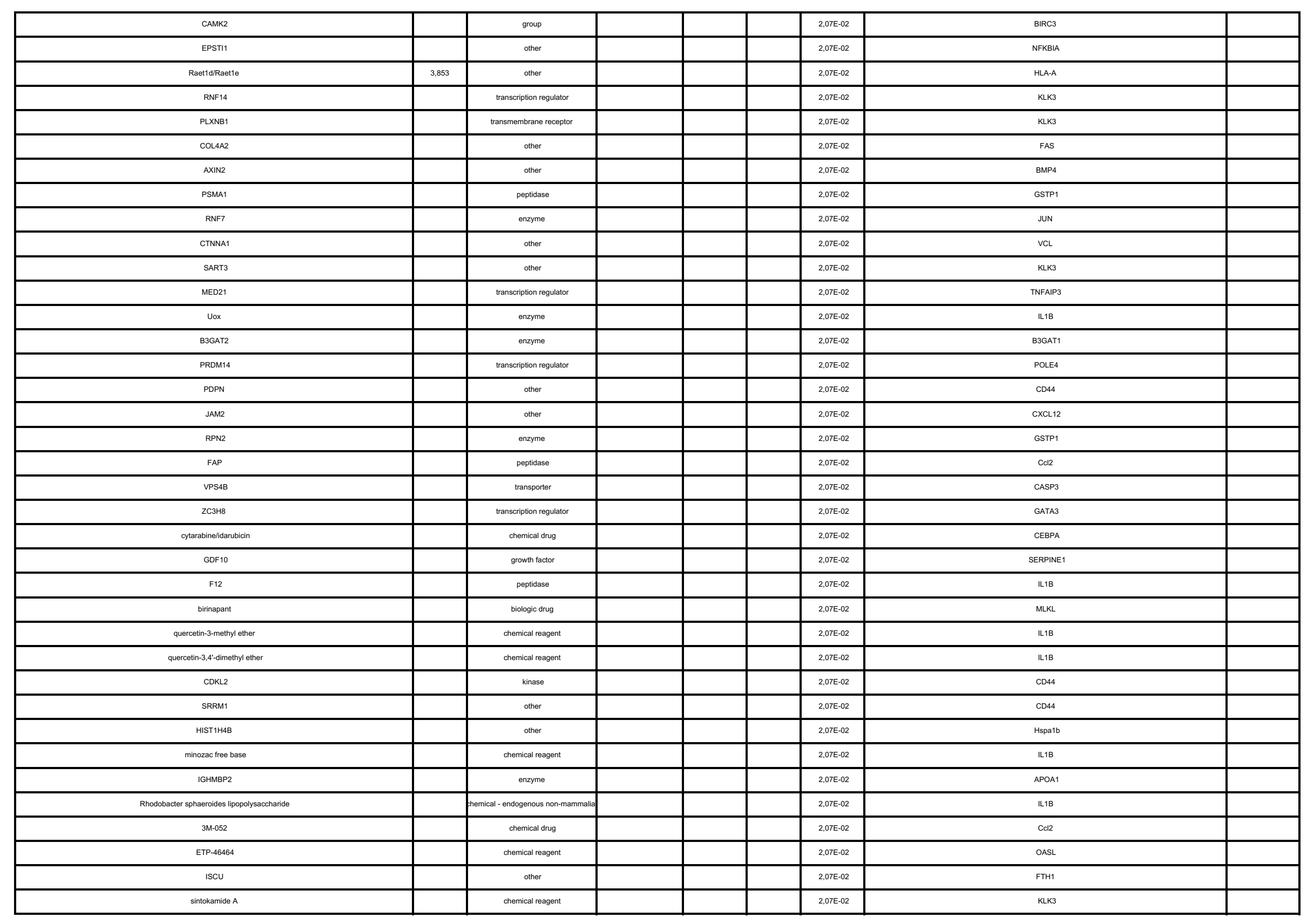




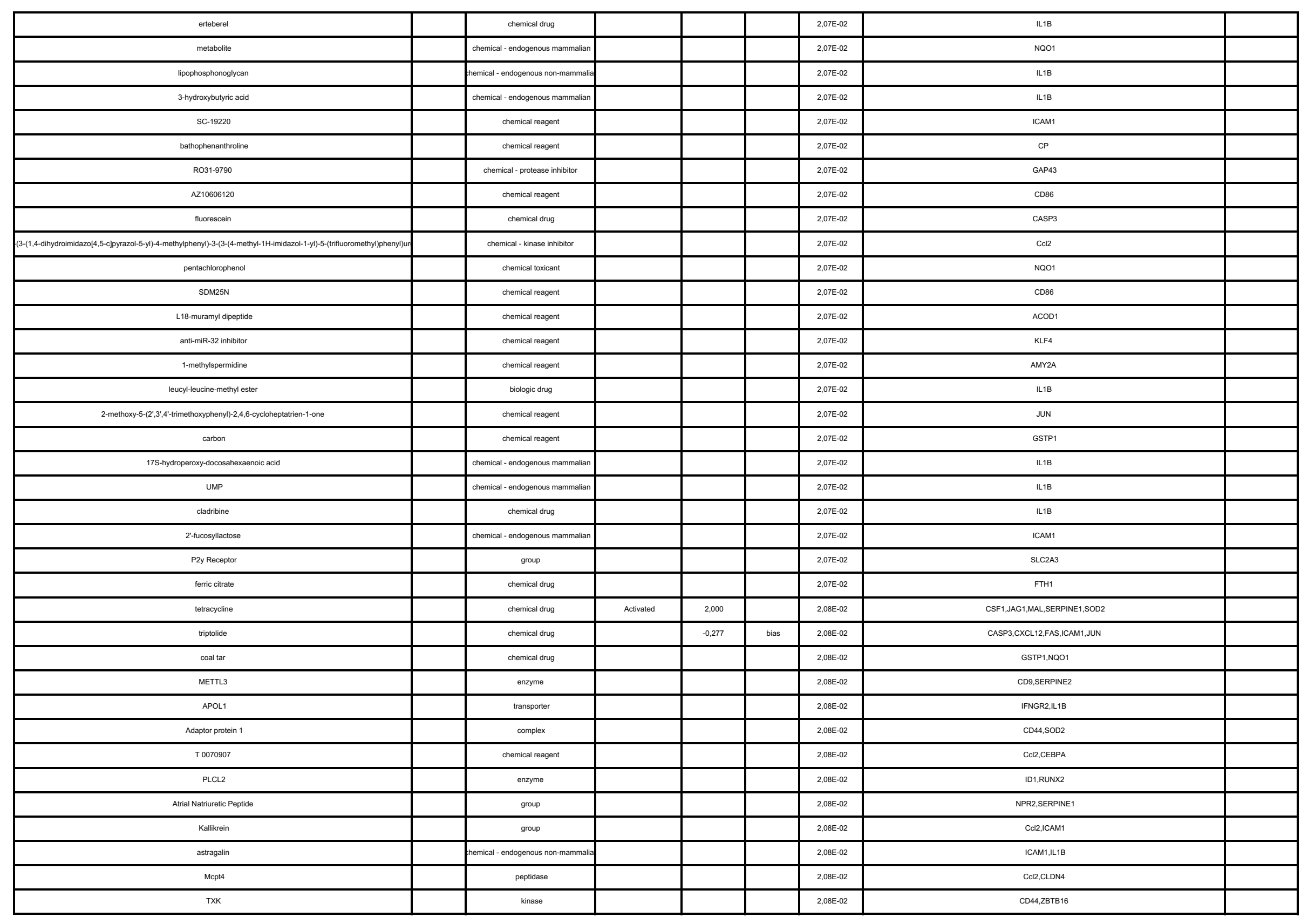




\begin{tabular}{|c|c|c|c|c|c|c|}
\hline DYRK1B & kinase & & & $2,08 E-02$ & CP,SOD2 & \\
\hline mir-208a-3p (and other miRNAs w/seed UAAGACG) & mature microRNA & & & $2,08 E-02$ & CD14,RUN $\times 2$ & \\
\hline MAFF & transcription regulator & & & $2,08 E-02$ & GSTP1,NQO1 & \\
\hline LL7RB & transmembrane receptor & & & $2,08 E-02$ & 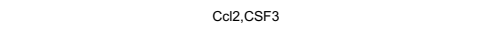 & \\
\hline VLDLR & transporter & & & $2,08 E-02$ & SERPINE1,TNFAIP3 & \\
\hline ENTPD1 & enzyme & & & $2,08 E-02$ & CD86,SERPINE1 & \\
\hline CHRNA3 & transmembrane receptor & & & $2,08 E-02$ & САРPЗ,GATA3 & \\
\hline НІІтензС & other & & & $2,08 E-02$ & IL1B,RND3 & \\
\hline sevoffurane & chemical drug & & & $2,08 E-02$ & CASP3, L11B & \\
\hline ferric ammonium citrate & chemical drug & & & $2,08 E-02$ & FTH1,FTL & \\
\hline 8-chlorophenythio-adenosine 3;5'-cyclic monophosphate & chemical - kinase inhibitor & 1,982 & bias & 2,12E-02 & BIRC3,СASP3,CEBPD,LPIN2 & \\
\hline IFN type 1 & group & 1,969 & bias & 2,12E-02 & COL12,F116, OASL,XDH & \\
\hline EPHB4 & kinase & 1,000 & bias & 2,12E-02 & BMP2,BMP4,GATA3,NeS & \\
\hline IGFBP2 & other & & & 2,12E-02 & CASP3,CHD, GDF15,PTGFRN & \\
\hline ARNTL & transcription regulator & 0,591 & bias & 2,12E-02 & CEBPA,HMGCS2,LL1B,NQO1 & \\
\hline LMNB1 & other & & & 2,12E-02 & 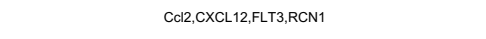 & \\
\hline brefeldin $A$ & hemical - endogenous non-mammalia & $-0,130$ & & $2,12 E-02$ & CD44,GADD45B,HLA-A,KLF4 & \\
\hline GRP & growth factor & & & $2,17 E-02$ & CTRB2,JUN,KLK3 & \\
\hline ACTLA & other & & & $2,17 E-02$ & CCNA2,FAS,SFN & \\
\hline DLL1 & enzyme & & & $2,17 E-02$ & CDB6,GATA3,JAG1 & \\
\hline CHADL & other & & & $2,17 E-02$ & CCl2,FAS,STEAP4 & \\
\hline CFLAR & other & & & $2,17 E-02$ & CSF3,GATA3,L1B & \\
\hline PD 123319 & chemical drug & & & $2,17 E-02$ & COIZ,CYBA, SERPINE1 & \\
\hline chysotile asbestos & chemical toxicant & & & $2,17 E-02$ & CLCA,CTSZ,LL1B & \\
\hline deoxynivalenol & chemical toxicant & & & $2,17 E-02$ & CLDNA,ICAM1,LL1B & \\
\hline stearic acid & chemical- endogenous mammalian & & & $2,17 E-02$ & ICAM, 1,L1B,NFKB|A & \\
\hline GnRH analog & biologic drug & 0,577 & & $2,18 \mathrm{E}-02$ & 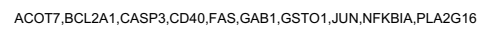 & \\
\hline GNA12 & enzyme & 0,447 & bias & $2,19 E-02$ & CCNA2,GSTA3,GSTP1,LAMB3,VCL & \\
\hline PAX3 & transcription regulator & & & $2,21 E-02$ & BMP4,FLT3, ID3, ILZR,LAMMB3,MEST,PTPN 14,PXDN,SFN & \\
\hline FOXP3 & transcription regulator & 0,447 & bias & $2,211-02$ & HLA-A,IGSF3, LIRLL, ILTR,LATS2,TNFAIP3 & \\
\hline SRC (family) & group & 1,969 & bias & $2,26 E-02$ & BMP2, СCl2, ICAM1, ID1 & \\
\hline RTN4 & other & & & $2,26 \mathrm{-}-02$ & GAP43,JUN,NQO1,Tpm1 & \\
\hline $\mathrm{CCN}_{2}$ & growth factor & 1,148 & bias & $2,31 E-02$ & BCLZA1, CDA4,JUN,SDC4,SERPRE 1,Tpm1 & \\
\hline RXRA & \begin{tabular}{|l|l|} 
ligand-dependent nuclear r receptor \\
\end{tabular} & 0,000 & & 2,33E-02 & 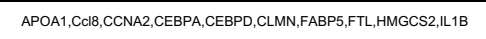 & \\
\hline etanercept & biologic drug & & & $2,37 E-02$ & СD83,ІСАM1,L18 & \\
\hline LPIN1 & phosphatase & & & $2,37 \mathrm{E}-02$ & CEBPA,IL1B,LPIN2 & \\
\hline indican & chemical - endogenous mammalian & & & $2,37 E-02$ & GATA3,ICAM1,L11B & \\
\hline
\end{tabular}




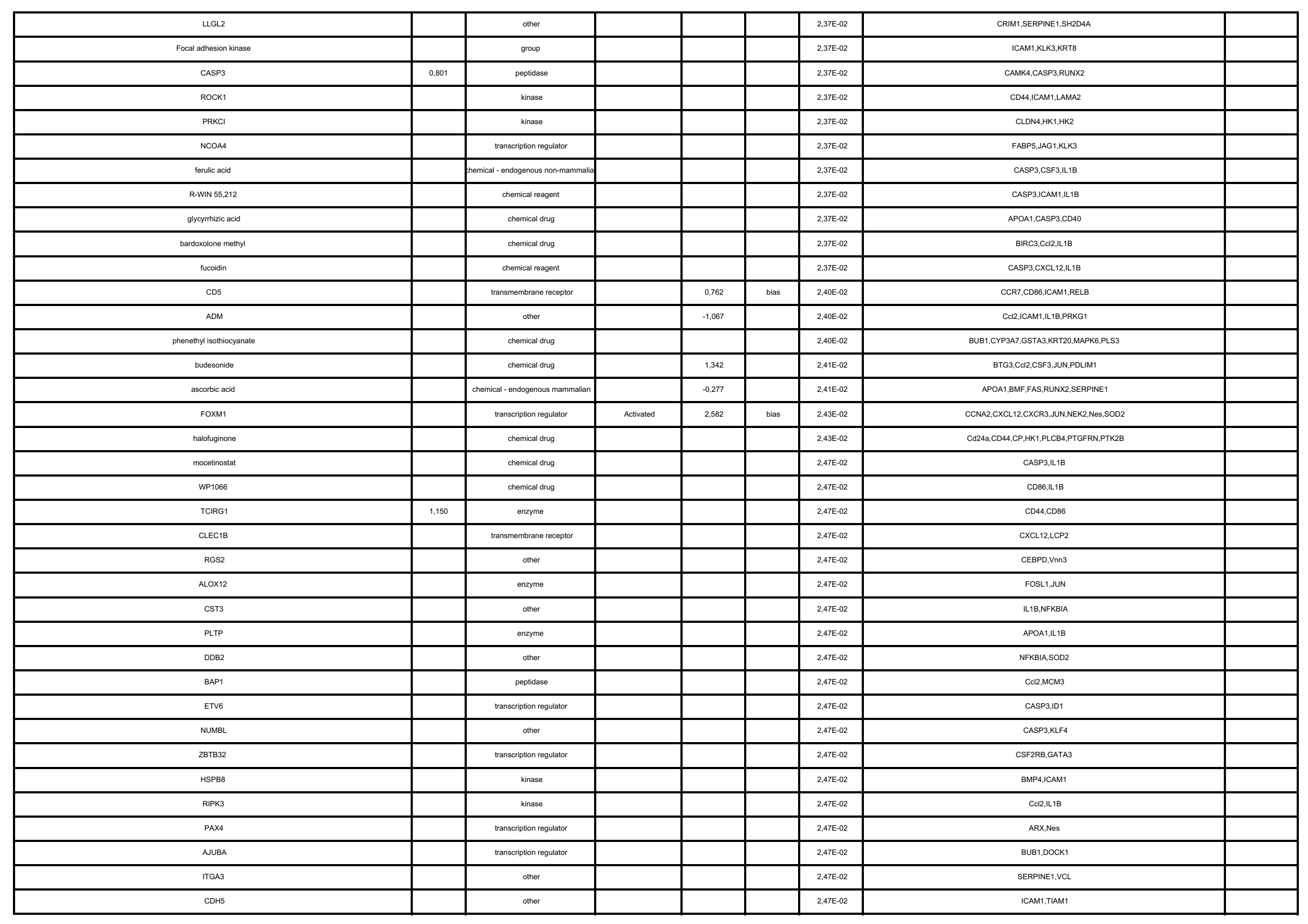




\begin{tabular}{|c|c|c|c|c|c|c|c|c|}
\hline IREB2 & & translation regulator & & & & $2,47 E-02$ & FTH1,FTL & \\
\hline ELN & & other & & & & $2,47 E-02$ & CD86,LL1B & \\
\hline PLCL1 & & enzyme & & & & $2,47 E-02$ & ID1,RUNX2 & \\
\hline ACO1 & & enzyme & & & & $2,47 E-02$ & FTH1,FTL & \\
\hline pentylenetetrazol & & chemical drug & & & & $2,47 E-02$ & L1B,JUN & \\
\hline toremifene & & chemical drug & & & & $2,47 E-02$ & CD86, KLK3 & \\
\hline D-sphingosine & & chemical-endogenous mammalian & & & & $2,47 E-02$ & CCI2,SERPINE1 & \\
\hline КT5720 & & chemical -kinase inhibitor & & & & $2,47 \mathrm{E}-02$ & NFKBAA,PRKG1 & \\
\hline tin protoporphyrin IX & & chemical reagent & & & & $2,47 E-02$ & CD86,Hamp/Hamp2 & \\
\hline cucurbitacin B & & hremical - endogenous non-mammalia & & & & $2,47 E-02$ & CASP $3, \mathrm{CCNA} 2$ & \\
\hline fatty acid & & chemical-endogenous mammalian & & 1,519 & bias & $2,50 \mathrm{E}-02$ & APOA1,CC12,L118,LPIN2,NFKBBA,SERPINE1 & \\
\hline TET2 & & enzyme & & $-0,378$ & bias & $2,51 \mathrm{E}-02$ & CA12,CLEC4D, IF16,IL18,PLS3,RND3,ZZTB16 & \\
\hline NRAA1 & & ligand-dependent nuclear recepptor & & $-1,615$ & bias & $2,53 \mathrm{E}-02$ & 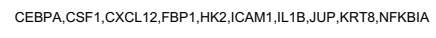 & \\
\hline R5020 & & chemical reagent & & 1,969 & bias & 2,53E-02 & BIRC3,CEBPA,LAMC2,NFKBIA,ZBTB16 & \\
\hline СDK9 & & kinase & & & & $2,55 E-02$ & ALAS1,NFKBIA,STC2,TNFAP3 & \\
\hline ZEB2 & & transcription regulator & & $-1,000$ & bias & $2,55 E-02$ & GZMA,ILRR,KRT19,TCT7 & \\
\hline АКT2 & & kinase & & & & $2,55 \mathrm{E}-02$ & BUB1,CC21,1RAK3,NQO1 & \\
\hline vav1 & & transcription regulator & & & & $2,59 \mathrm{E}-02$ & BCL2A1,JUN,RALA & \\
\hline IFRD1 & & other & & & & $2,59 \mathrm{E}-02$ & CTSZ,HK1,LAMA2 & \\
\hline THBS4 & & other & & & & $2,59 \mathrm{E}-02$ & CCl2,CD40,ICAM1 & \\
\hline fluvoxamine & & chemical drug & & & & $2,59 \mathrm{E}-02$ & CCR7,HTR7,RACK1 & \\
\hline ERN1 & & kinase & & 1,404 & bias & $2,60 \mathrm{E}-02$ & ANG,CCI2,CEBPD,FAS, IL1B,JUN,TINAGL1 & \\
\hline SPARC & & other & & $-1,353$ & & $2,60 \mathrm{E}-02$ & CEBPD,CLDNA,NOTCHZ,PLET1,SERPINE1,SLC3AA2 & \\
\hline GAST & & other & & 1,342 & bias & $2,65 E-02$ & Hamp/Hamp2,JAG1,JUN,MCM3,NFKB|A & \\
\hline berberine & & chemical drug & & 0,686 & bias & $2,71 E-02$ & BIRC3,CASP3,СD40,СD86,ICAM1,L11 18 & \\
\hline alefacept & & biologic drug & & $-1,982$ & bias & $2,71 \mathrm{E}-02$ & CD2,CDBA,СYBA,ILR & \\
\hline oblimersen & & biologic drug & Activated & 2,000 & bias & $2,71 E-02$ & CEBPD,GADD45B,IF116,OASL & \\
\hline CAT & & enzyme & & $-1,067$ & bias & $2,71 E-02$ & BCL2A1,CL12,CLCA1, SERPINE1 & \\
\hline PLAG1 & & transcription regulator & & 0,000 & bias & $2,71 E-02$ & KRT19,LLRB,PIR,PPIC & \\
\hline mir-24 & & microRNA & & $-0,404$ & & $2,71 E-02$ & CXCR3,GATA3,SERPINE1,TCC7 & \\
\hline CLEC11A & & growth factor & & & & $2,71 E-02$ & CXCL12,L118,RUNX2,ZYX & \\
\hline anisomycin & & hremical- endogenous non-mammalia & & 1,966 & bias & $2,71 E-02$ & LL1RL1,JUN,SDC1,SERPINE1 & \\
\hline SP110 & 2,929 & transcription regulator & & $-0,378$ & & $2,78 E-02$ & BMP4,DMD,PANX1,PERP, RIPK2,TAB3,YYAP1 & \\
\hline CARD9 & & other & & & & $2,81 E-02$ & CC12,CSF3,L1B & \\
\hline $\mathrm{IHH}$ & & enzyme & & & & $2,81 E-02$ & BMPA,RUNX2,SMO & \\
\hline PRKCZ & & kinase & & & & $2,81 \mathrm{E}-02$ & CASP3,JUN,NFKBAA & \\
\hline BECN1 & & other & & & & $2,81 E-02$ & BMF,IL1B,SOD2 & \\
\hline
\end{tabular}




\begin{tabular}{|c|c|c|c|c|c|c|c|}
\hline CORT & & other & & & $2,81 E-02$ & 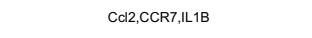 & \\
\hline N-(3-(aminomethy)|benzyy)acetamidine & & chemical reagent & & & $2,81 \mathrm{E}-02$ & CASP3,FAS,RIPK2 & \\
\hline hymecromone & & chemical drug & & & $2,81 E-02$ & CASP $3, \mathrm{CD} 44, \mathrm{FAS}$ & \\
\hline IFNA1/FNA13 & & cyrokine & 1,131 & bias & $2,87 E-02$ & CD40, СD83,СD86, L1118 & \\
\hline EB13 & & cytokine & 1,982 & bias & $2,87 E-02$ & CD86,HLA-A,ICAM1,LAG3 & \\
\hline MDGA2 & & other & & & $2,88 E-02$ & BCLLA1,FAS & \\
\hline $\mathrm{FH}$ & & enzyme & & & $2,88 E-02$ & LTBP2,NQO1 & \\
\hline CRBN & & enzyme & & & $2,88 E-02$ & แ1B,JUN & \\
\hline harmine & & hemical - endogenous non-mammalia & & & $2,88 E-02$ & BMP2,RUN×2 & \\
\hline sox5 & & transcription regulator & & & $2,88 E-02$ & BMPG,RUNX2 & \\
\hline miR-210-3p (miRNAs w/sed UGUGCGU) & & mature microRNA & & & $2,88 E-02$ & FGFRL1,RAD52 & \\
\hline mir-127 & & microRNA & & & $2,88 E-02$ & LL1B,MRC1 & \\
\hline HLA-A & 1,061 & other & & & $2,88 E-02$ & HLA-A,LAG3 & \\
\hline XRCC5 & & enzyme & & & $2,88 E-02$ & Кடкз,ХDH & \\
\hline CD180 & & other & & & $2,88 E-02$ & BCL2A1,CDB6 & \\
\hline NLRC4 & & other & & & $2,88 E-02$ & CD86,LL1B & \\
\hline FRS2 & & other & & & $2,88 \mathrm{E}-02$ & BMP4,CD44 & \\
\hline PLAZC2E & & enzyme & & & $2,88 E-02$ & IL1B,SERPINE1 & \\
\hline UGCG & & enzyme & & & $2,88 E-02$ & CASPB, ІСAM1 & \\
\hline PON1 & & phosphatase & & & $2,88 E-02$ & CD68,ICAM1 & \\
\hline SDC1 & 1,625 & enzyme & & & $2,88 E-02$ & CC12,GALNT3 & \\
\hline CRY1 & & enzyme & & & $2,88 E-02$ & СЕВРA,IL1B & \\
\hline СОК7 & & kinase & & & $2,88 \mathrm{E}-02$ & STC2,TNFAIP3 & \\
\hline DUSP14 & & phosphatase & & & $2,88 E-02$ & NFKBIZ,Vnn3 & \\
\hline BHLLE41 & & transcription regulator & & & $2,88 E-02$ & GATA3,HK2 & \\
\hline HSPA1AHSPA1B & & enzyme & & & $2,88 E-02$ & Hspa1b,L118 & \\
\hline 23-cyclic guanosine monophosphate-adenosine monophosphate & & chemical-endogenous mammalian & & & $2,88 E-02$ & CD86,LL1B & \\
\hline bisphenol A diglycidyl ether & & chemical reagent & & & $2,88 E-02$ & ICAM1,SERPINE1 & \\
\hline RV 538 & & chemical reagent & & & $2,88 E-02$ & ІСАM, ІL1B & \\
\hline ethylenediaminetetracetic acid & & chemical drug & & & $2,88 \mathrm{E}-02$ & BCL2A1,L1B & \\
\hline irinotecan & & chemical drug & & & $2,88 E-02$ & FAS,LL1B & \\
\hline isatoribine & & chemical reagent & & & $2,88 E-02$ & $\mathrm{CD} 40, \mathrm{CD} 86$ & \\
\hline cyclomaltodextrin & & chemical drug & & & $2,88 E-02$ & CSF3,L1B & \\
\hline topiramate & & chemical drug & & & $2,88 E-02$ & CASP3,L1B & \\
\hline CNR1 & & G-protein coupled receptor & 0,420 & bias & $2,88 E-02$ & BMP2,BMP4,CO12,CC18, CORO2A,NOD2,PTK2B & \\
\hline ZAP70 & & kinase & 1,982 & bias & $3,04 E-02$ & ALAS1,ANG, CCR7,CYBA & \\
\hline ЕLK1 & & transcription regulator & 1,951 & bias & $3,04 E-02$ & BMP2,FOSL1,JUN,RUNX2 & \\
\hline
\end{tabular}




\begin{tabular}{|c|c|c|c|c|c|c|}
\hline Pln & other & & & 3,04E- -02 & APOA1,RACK1,RALA,RRAS & \\
\hline $\mathrm{FBXO} 2$ & enzyme & 1,109 & & $3,04 E-02$ & IL1B,KRT19, OAT,SOD2,TCF7 & \\
\hline зм-011 & chemical reagent & & & $3,05 E-02$ & $\mathrm{CD40,CD83,CD86}$ & \\
\hline MALAT1 & other & & & $3,05 E-02$ & COL2,FRMD8,Glycam1 & \\
\hline KEAP1 & transcription regulator & & & $3,05 E-02$ & GSTP1,LL1B,NQO1 & \\
\hline PDPK1 & kinase & & & 3,05E-02 & CCR7,LL1B,SERPINE1 & \\
\hline TIMP1 & cytokine & & & $3,05 E-02$ & A4GALT,CD40,CD44 & \\
\hline lactic acid & chemical-endogenous mammalian & & & $3,05 E-02$ & CXCL12,LL1B,MRC1 & \\
\hline bafiliomycin $\mathrm{A}_{1}$ & chemical drug & & & 3,05E-02 & CD40,CD9,NFKBIA & \\
\hline TGFB3 & growth factor & 1,709 & bias & $3,15 E-02$ & ADAM19,GATA3,JUN,LTBP2,SERPINE1,SERPINE2 & \\
\hline lithium & chemical drug & $-0,447$ & & 3,18E-02 & CAMK4,CASP3,FAS,JUN,NCS1 & \\
\hline FBXW7 & enzyme & $-0,577$ & bias & $3,21 E-02$ & HSPB 1,JUN,NQO1, SERPINE1 & \\
\hline POUZAF1 & transcription regulator & & & $3,21 E-02$ & B4GALT1,CD86, ID3,LCK & \\
\hline cadmium chloride & chemical toxicant & & & $3,21 E-02$ & CXCL12,HSPB1,JUN,KLK3 & \\
\hline Secretase gamma & complex & & & $3,29 E-02$ & BATF,CDB3,CD86 & \\
\hline ZBEDG & transcription regulator & & & $3,29 \mathrm{E}-02$ & CRIM1,UPP1,WWC1 & \\
\hline CHRNA7 & transmembrane receptor & & & $3,29 E-02$ & $\mathrm{CCl} 2,1 \mathrm{DD}, \mathrm{LL1B}$ & \\
\hline ANXA2 & other & & & $3,29 \mathrm{E}-02$ & ANG,FAS,JUN & \\
\hline DNASE2 & enzyme & & & $3,29 E-02$ & CP,HampHHamp2,Ms44atb (includes others) & \\
\hline SELP & transmembrane receptor & & & $3,29 E-02$ & BCL2A1,BST1,IL1B & \\
\hline PRMT1 & enzyme & & & $3,29 \mathrm{E}-02$ & FTH1,FTL,MRC1 & \\
\hline zвтв7в & transcription regulator & & & $3,29 \mathrm{E}-02$ & CDBA,GATA3,KLF4 & \\
\hline PLAT & peptidase & & & $3,29 E-02$ & แL1B,JUN,SERPINE 1 & \\
\hline TYK2 & kinase & & & $3,29 E-02$ & CSF3,Hamp/Hamp2,L11B & \\
\hline CDC42 & enzyme & & & $3,29 E-02$ & CASP3,CD86,JUN & \\
\hline MEIS1 & transcription regulator & & & $3,29 \mathrm{E}-02$ & CHD9,FLT3,RUNX2 & \\
\hline S-nitroso-N-acetypenicillamine & chemical reagent & & & $3,29 \mathrm{E}-02$ & CXCL12,FAS,PRKG1 & \\
\hline glycochenodeoxycholate & chemical- endogenous mammalian & & & $3,31 E-02$ & BCL2A1,BIRC3 & \\
\hline MEG3 & other & & & $3,31 \mathrm{E}-02$ & GDF15,JUN & \\
\hline Rac & group & & & $3,31 E-02$ & BMP2,CD9 & \\
\hline belnacasan & chemical drug & & & $3,31 \mathrm{E}-02$ & $\mathrm{CC2} 2,111 \mathrm{~B}$ & \\
\hline SGPP1 & phosphatase & & & $3,31 E-02$ & IL1B,тGM3 & \\
\hline NFKBID & transcription regulator & & & $3,31 E-02$ & CD86,CD9 & \\
\hline TNFAIPBL2 & other & & & $3,31 E-02$ & $\mathrm{CC} 2,111 \mathrm{~B}$ & \\
\hline CBSICBSL & enzyme & & & $3,31 E-02$ & APOA 1, CASP 3 & \\
\hline TAC4 & other & & & $3,31 \mathrm{E}-02$ & CD86,IL1B & \\
\hline $\operatorname{cox} 411$ & enzyme & & & $3,31 E-02$ & SLC24A3,TSPAN8 & \\
\hline
\end{tabular}




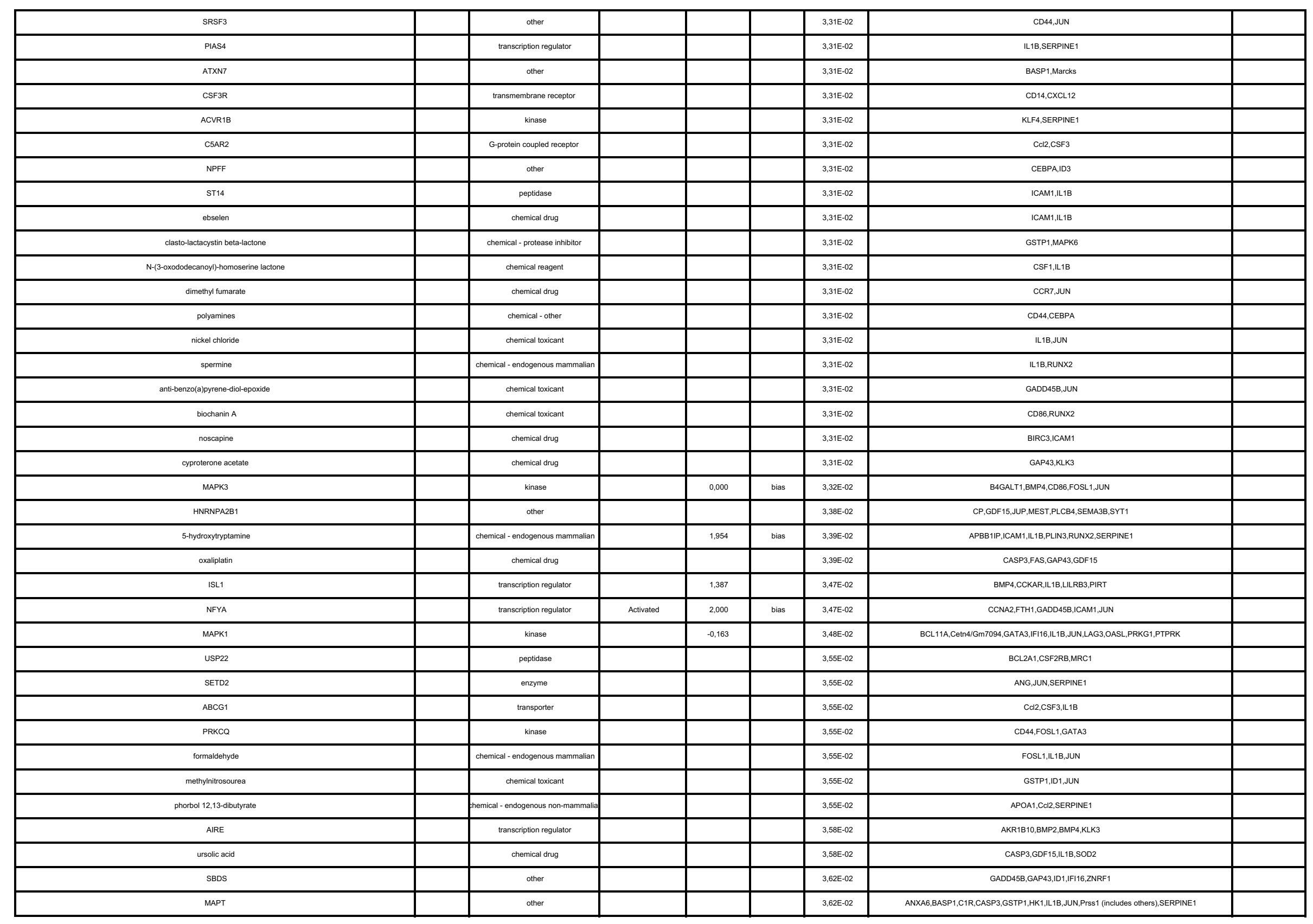




\begin{tabular}{|c|c|c|c|c|c|c|}
\hline MAPAK4 & kinase & Inhibited & $-2,449$ & bias & 3,64E-02 & ALDH3B1,CEBPA,HGPD,MGLL,PLCB4,ST3GAL4 \\
\hline BDNF & growth factor & & 1,779 & bias & $3,64 \mathrm{E}-02$ & АСОT7,BMP2, СASP, ,CAV2,CP, FTL,GAP43,JUN,SEMABE,SOO2 \\
\hline STATSB & transcription regulator & & 0,302 & & $3,70 \mathrm{E}-02$ & BIRC3,BMF,СASP3,FAS, GADD45B, GIycam1,GSTP1, Нamp/Hamp2,KRT19,NQO1 \\
\hline Lh & complex & & 0,911 & bias & 3,72E-02 & DHRS3,HK2,MAPKG,PLIN3,RUNX2,SOD2, TNFAP3, TNFRSF 12A,UPP1,VCL \\
\hline cyclic GMP & chemical-endogenous mammalian & & & & $3,77 E-02$ & SERPINE1,VCL \\
\hline fluoride & chemical-endogenous mammalian & & & & 3,77E-02 & FAS,RUNX2 \\
\hline lonataranib & chemical drug & & & & $3,77 \mathrm{E}-02$ & BCL2A1,BIRC3 \\
\hline PPARa-RXRa & complex & & & & 3,77E-02 & APOA1,NFKBIA \\
\hline CLCN5 & ion channel & & & & 3,77E-02 & CC12,SLC3AA2 \\
\hline IGFBP7 & transporter & & & & $3,77 \mathrm{E}-02$ & CASP3,SOD2 \\
\hline TAP1 & transporter & & & & 3,77E-02 & HLA-A,ILL1B \\
\hline AIMP1 & cytokine & & & & 3,77E-02 & ICAM1,L1B \\
\hline PNN & other & & & & $3,77 E-02$ & GDF15,JUN \\
\hline CAPN3 & peptidase & & & & 3,77E-02 & FTH1,SOD2 \\
\hline SLC2A1 & transporter & & & & 3,77E-02 & NQO1,SERPINE1 \\
\hline BDKRB2 & G-protetin coupled receptor & & & & $3,77 E-02$ & CD44,ICAM1 \\
\hline TREX1 & enzyme & & & & $3,77 E-02$ & IFI16,OASL \\
\hline PARK7 & enzyme & & & & $3,77 E-02$ & ICAM1,SOD2 \\
\hline PDE5A & enzyme & & & & $3,77 E-02$ & IL1B,PRKG1 \\
\hline SPINT1 & other & & & & 3,77E-02 & PTK7,TCF \\
\hline$\llcorner-685,458$ & chemical - protease inhibitor & & & & $3,77 E-02$ & ID1,PTPRK \\
\hline Mn(III)-tetrakis-(4-benzoic acid) porphyrin & chemical reagent & & & & $3,77 E-02$ & CLDN4,SERPINE1 \\
\hline lauric acid & chemical-endogenous mammalian & & & & $3,77 E-02$ & $\mathrm{CD} 40, \mathrm{CD} 86$ \\
\hline ILR1 & transmembrane receptor & & 1,718 & bias & $3,77 E-02$ & CO12,CXCL12,ICAM1,L1B \\
\hline FL1 & transcription regulator & & 1,969 & bias & $3,77 \mathrm{E}-02$ & $\mathrm{CCl} 2, \mathrm{CSF} 3, \mathrm{CXCR} 3, \mathrm{RACK} 1$ \\
\hline 8-bromoguanosine 3;5-cyclic monophosphate & chemical - kinase inhibitor & & & & $3,81 \mathrm{E}-02$ & BMP2,CD8A,SLC12A4 \\
\hline TARDBP & transcription regulator & & & & $3,81 \mathrm{E}-02$ & САSP3, СС2, IL1B \\
\hline IGFBP3 & other & & & & $3,81 E-02$ & ICAM1,L1B,SERPINE1 \\
\hline GTF2B & transcription regulator & & & & $3,81 \mathrm{E}-02$ & BCL2A1,NFKBAA,TNFAP3 \\
\hline parthenolide & chemical drug & & & & $3,81 E-02$ & $\mathrm{CCl} 2,1118, \mathrm{SOD} 2$ \\
\hline $\mathrm{ZFH} \times 3$ & transcription regulator & & & & $3,93 E-02$ & APOA1,GSTP1,HLA-A,ID3,Nes \\
\hline STK11 & kinase & & $-0,711$ & & 3,94E-02 & ALCAM, CCl2, CCNA2,CSF3,DOCK4,FOSL1, GADD458, ID1,RASGRP1,SERPINE1 \\
\hline 3-deazaneplanocin & chemical drug & & 0,687 & bias & $3,96 E-02$ & FAS,KLF, LAMB3,NOTCH2 \\
\hline ЧСНL1 & peptidase & & 1,000 & & 3,96E-02 & ANXAG,DOCK10,MAPKG,SHZ204A \\
\hline СТкзв & kinase & Activated & 2,433 & & $4,04 E-02$ & BCL2A1,BRC3,CC12,IL1B,NFKBIZ,SERPINE1 \\
\hline sox4 & transcription regulator & Activated & 2,813 & bias & 4,05E-02 & $2610035017 \mathrm{1RR}, \mathrm{CD} 2, \mathrm{LCK}, \mathrm{PPIC}, \mathrm{RNASE} 4, \mathrm{SERP}$ INE2, TAX1BP3,TIAMM \\
\hline POUZF2 & transcription regulator & Activated & 2,000 & bias & 4,09E-02 & DEPP1,FJX1,JUN,LCK,TNFRSF12A \\
\hline
\end{tabular}




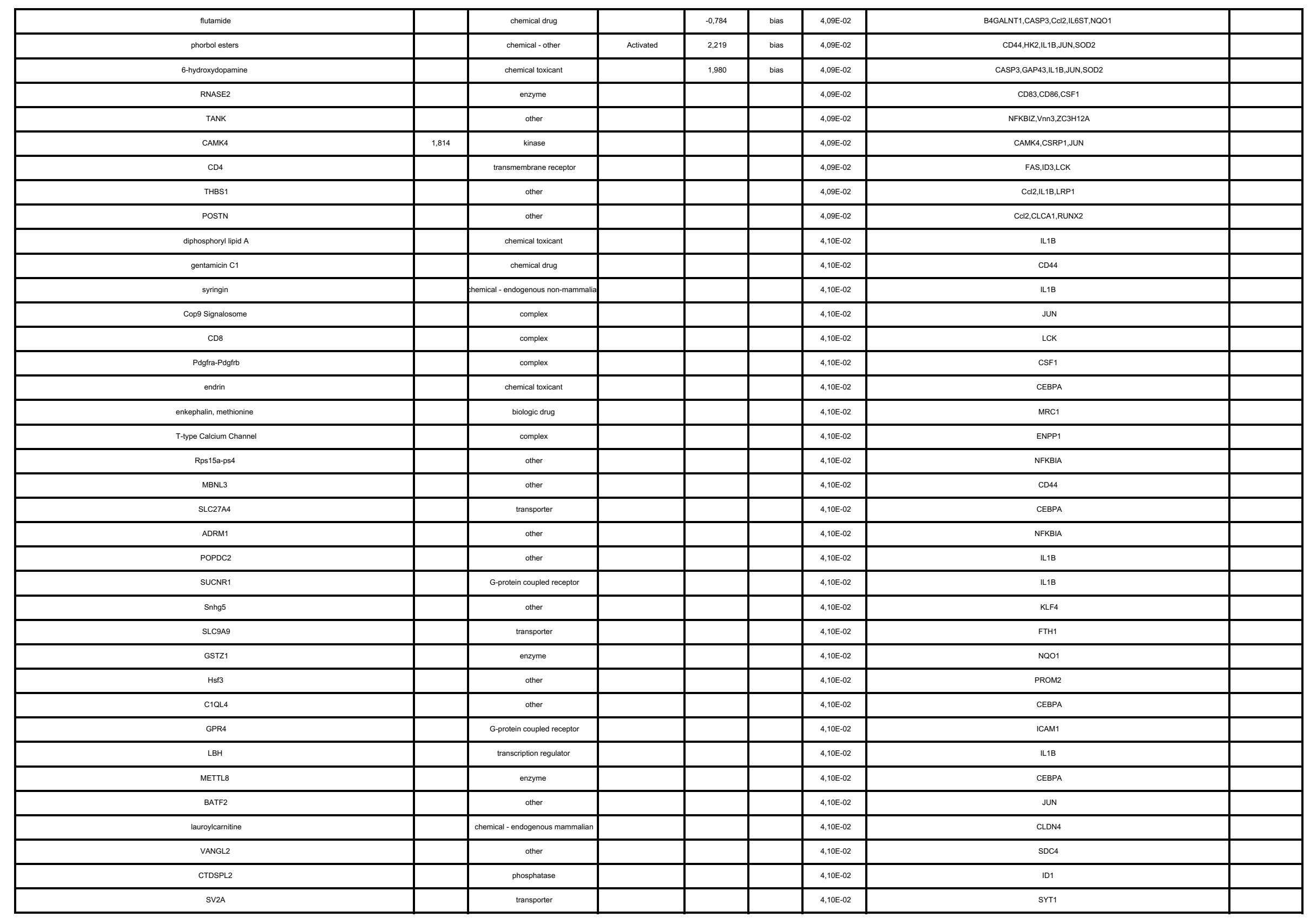




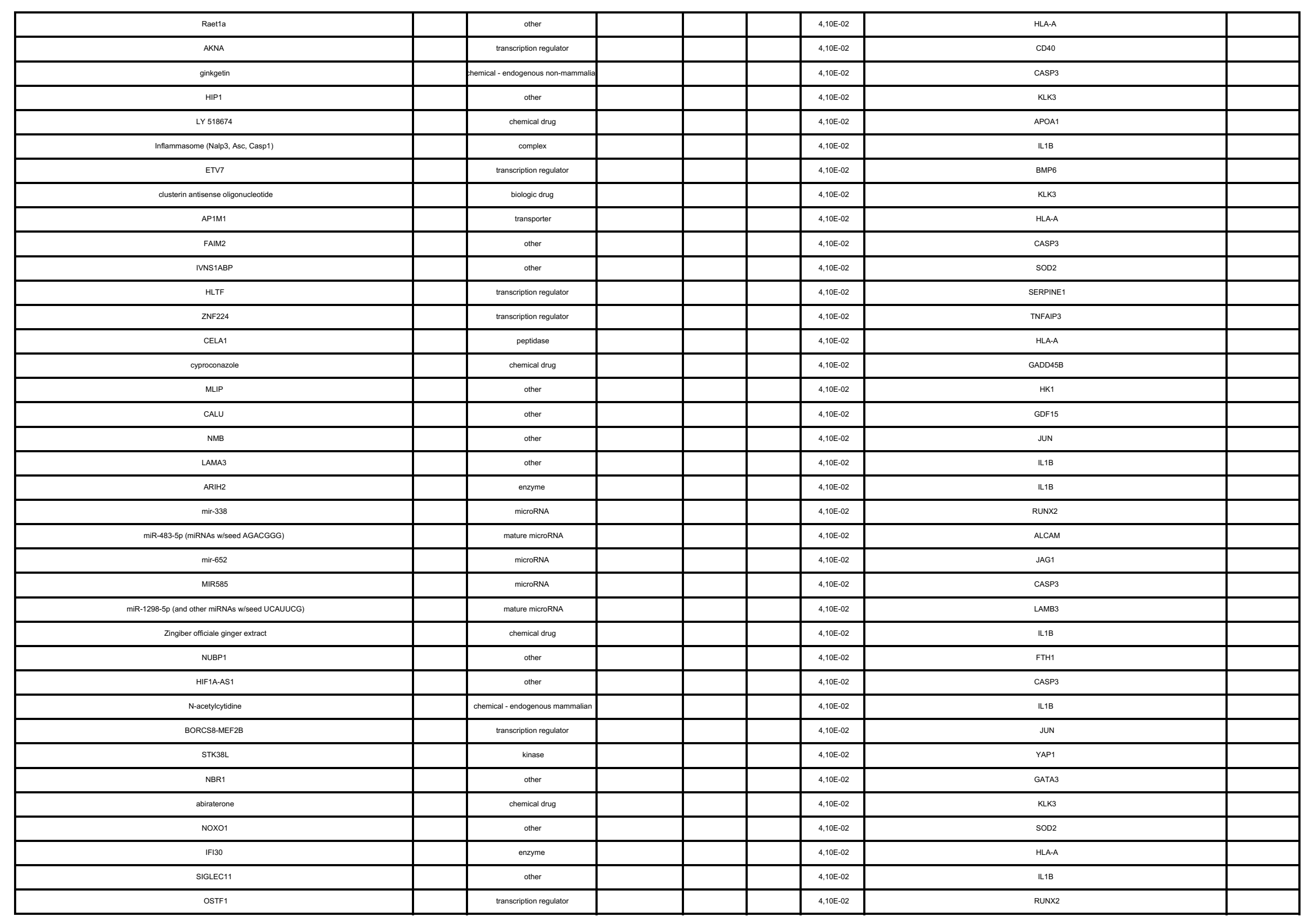




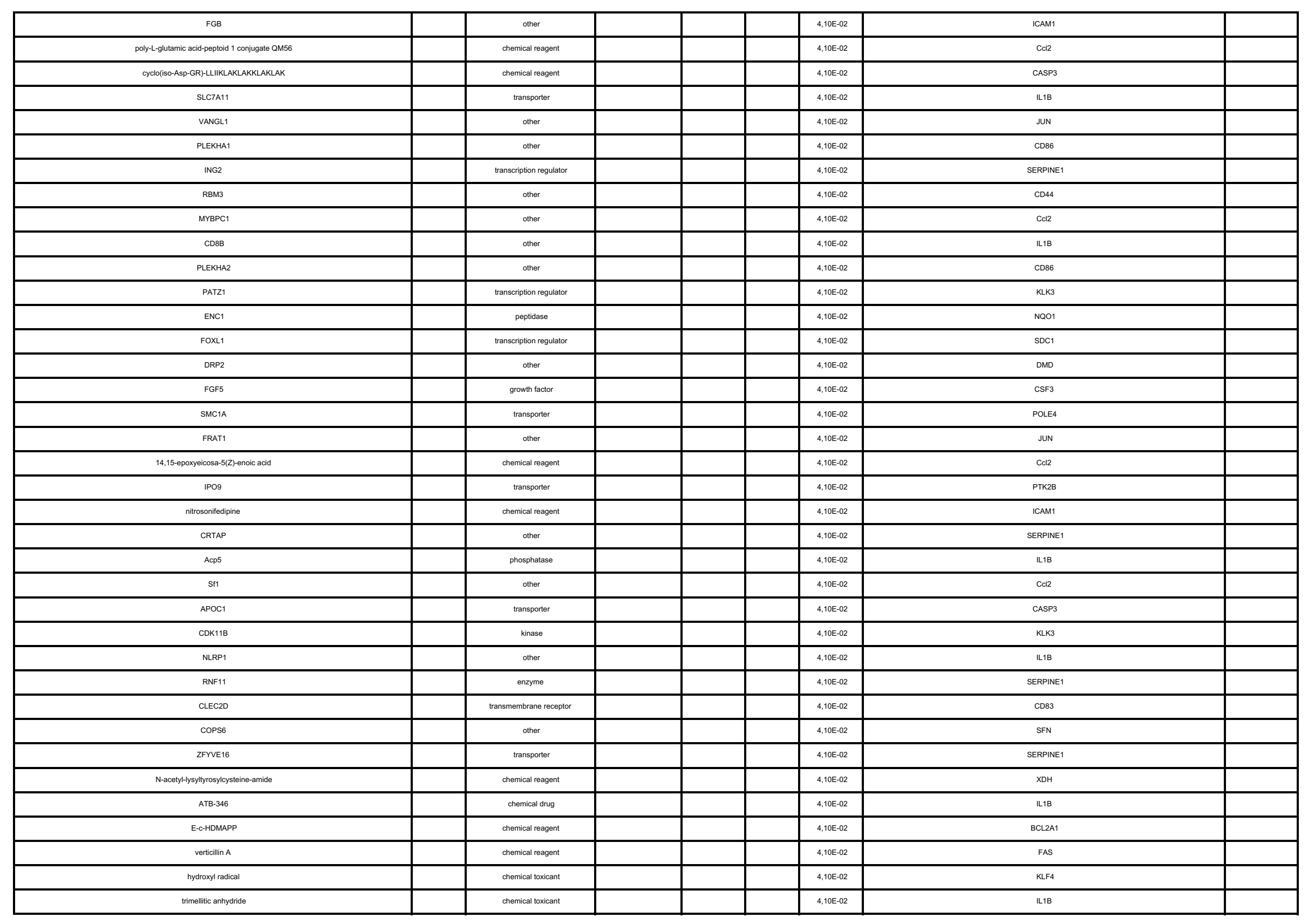




\begin{tabular}{|c|c|c|c|c|c|c|}
\hline amsacrine & chemical drug & & & & $4,10 \mathrm{E}-02$ & JUN \\
\hline sarpogrelate & chemical drug & & & & $4,10 \mathrm{E}-02$ & SERPINE1 \\
\hline methiothepin & chemical drug & & & & $4,10 \mathrm{E}-02$ & SERPINE1 \\
\hline sibutramine & chemical drug & & & & $4,10 \mathrm{E}-02$ & SERPINE1 \\
\hline 2,5-hexanedione & chemical toxicant & & & & $4,10 \mathrm{E}-02$ & FAS \\
\hline hexaarginine-neomycin B conjugate & chemical reagent & & & & $4,10 \mathrm{E}-02$ & CDBA \\
\hline oxazepam & chemical drug & & & & $4,10 \mathrm{E}-02$ & GADD45B \\
\hline 3,3-'-minodipropionitilie & chemical toxicant & & & & $4,10 \mathrm{E}-02$ & $\mathrm{COl} 2$ \\
\hline gilclazide & chemical drug & & & & $4,10 \mathrm{E}-02$ & SLC2A3 \\
\hline chalcone-24 & chemical reagent & & & & $4,10 \mathrm{E}-02$ & BIRC3 \\
\hline trichosanthin & chemical drug & & & & $4,10 \mathrm{E}-02$ & JAG1 \\
\hline terpenoid & chemical - endogenous marmmalian & & & & $4,10 \mathrm{E}-02$ & LRP1 \\
\hline urushiol & hemical - endogenous non-mammalia & & & & $4,10 \mathrm{E}-02$ & М1в \\
\hline phenol & chemical - endogenous mammalian & & & & $4,10 \mathrm{E}-02$ & แ1в \\
\hline sodium fluoride & chemical drug & & & & $4,10 \mathrm{E}-02$ & ICAM1 \\
\hline cation & chemical - other & & & & $4,10 \mathrm{E}-02$ & GSTP1 \\
\hline echium oil & chemical drug & & & & $4,10 \mathrm{E}-02$ & L1в \\
\hline procaterol & chemical drug & & & & $4,10 \mathrm{E}-02$ & ICAM1 \\
\hline Z-YVADFMK & chemical reagent & & & & $4,10 \mathrm{E}-02$ & L1в \\
\hline verteporfin & chemical drug & & & & $4,10 \mathrm{E}-02$ & YAP1 \\
\hline КT 5926 & chemical - kinase inhibitor & & & & $4,10 \mathrm{E}-02$ & CD14 \\
\hline aluminum & chemical drug & & & & $4,10 \mathrm{E}-02$ & RUNX2 \\
\hline tyrphostin 856 & chemical -kinase inhibitor & & & & $4,10 \mathrm{E}-02$ & ICAM1 \\
\hline tibolone & chemical drug & & & & $4,10 \mathrm{E}-02$ & SERPINE1 \\
\hline flunisolide & chemical drug & & & & $4,10 \mathrm{E}-02$ & ICAM1 \\
\hline fluorocotitic acid & chemical reagent & & & & $4,10 \mathrm{E}-02$ & LL1B \\
\hline 1-phenazinecarboxylic acid & hemical - endogenous non-mammalia & & & & $4,10 \mathrm{E}-02$ & ICAM1 \\
\hline HOXD10 & transcription regulator & & 0,000 & & $4,17 E-02$ & CD44,ICAM1,NCS1,SERPINE1 \\
\hline rotenone & chemical toxicant & & 0,832 & & $4,17 \mathrm{E}-02$ & BIRC3,САSP, ,L118,SOD2 \\
\hline interferon beta-1a & biologic drug & & & & $4,17 E-02$ & GADD45B,GSTP1,LLRBB,MAL,NFKBIA,SERPING1 \\
\hline CREM & transcription regulator & & 1,131 & & $4,18 \mathrm{E}-02$ & САMK4,CD86, CD8A,GADD458,Marcks, MEST,TPm1 \\
\hline aldosterone & \begin{tabular}{|c|} 
chemical - endogenous mammalian \\
\end{tabular} & Activated & 2,578 & bias & $4,18 E-02$ & 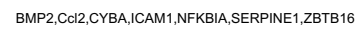 \\
\hline epothilone B & chemical drug & & & & $4,25 E-02$ & FAS,SFN \\
\hline selumetinib & chemical drug & & & & $4,25 \mathrm{E}-02$ & JUN,RUNX2 \\
\hline DPH5 & enzyme & & & & $4,25 E-02$ & LLB, NFKBBA \\
\hline MDK & growth factor & & & & $4,25 \mathrm{E}-02$ & Cald1,Ccl2 \\
\hline NUP62 & transporter & & & & 4,25E-02 & PERP,WWC1 \\
\hline
\end{tabular}




\begin{tabular}{|c|c|c|c|c|c|c|c|}
\hline LY96 & & transmembrane receptor & & & $4,25 E-02$ & $\mathrm{CD} 40, \mathrm{CD} 86$ & \\
\hline mir-204 & & microRNA & & & $4,25 E-02$ & LL1B,RuNX2 & \\
\hline FCGRIA & & transmembrane receptor & & & $4,25 E-02$ & CSF1,L1B & \\
\hline HDAC9 & & transcription regulator & & & $4,25 E-02$ & CEBPA,JUN & \\
\hline STIM1 & & ion channel & & & $4,25 E-02$ & GSTO1,NQO1 & \\
\hline 10-nitroleate & & chemical-endogenous mammalian & & & $4,25 E-02$ & COLI,ICAM1 & \\
\hline CR2 & & transmembrane receptor & & & $4,25 E-02$ & FAS,LL1B & \\
\hline BCL10 & & transcription regulator & & & $4,25 E-02$ & CD44,TNFAP3 & \\
\hline CNR2 & & G-protein coupled receptor & & & $4,25 E-02$ & $\mathrm{CC} 2,111 \mathrm{~B}$ & \\
\hline cux1 & & transcription regulator & & & 4,25E-02 & $\mathrm{CCl} 8, \mathrm{CD} 8 \mathrm{~A}$ & \\
\hline 101.10 peptide & & chemical reagent & & & $4,25 E-02$ & CC12,1118 & \\
\hline 3-aminobenzzamide & & chemical toxicant & & & $4,25 E-02$ & ICAM1,L1B & \\
\hline 2-acetylaminofluorene & & chemical toxicant & & & $4,25 E-02$ & JAG1, NOTCH2 & \\
\hline ally sulfide & & chemical drug & & & 4,25E-02 & CASP, GSTA3 & \\
\hline concanamycin $\mathrm{A}$ & & chemical drug & & & 4,25E-02 & ICAM1,NFKBIA & \\
\hline mevastatin & & chemical drug & & & $4,25 E-02$ & BMP4,KLF4 & \\
\hline indole & & \begin{tabular}{|c|} 
chemical - endogenous mammalian \\
\end{tabular} & & & $4,25 E-02$ & CGN,TPP3 & \\
\hline farmesyl pyrophosphate & & chemical-endogenous mammalian & & & $4,25 E-02$ & ICAM1,SERPINE1 & \\
\hline tetrandrine & & chemical drug & & & $4,25 \mathrm{E}-02$ & CASPB,JUN & \\
\hline REST & & transcription regulator & $-1,400$ & bias & $4,30 \mathrm{E}-02$ & B3GAT1, СEBPA,CXCL12,GAP43,Nes, OAT,SYT1 & \\
\hline arsenic trixide & & chemical drug & 1,672 & & 4,34E-02 & BCL2A1,CD44,FAS,FTH1,MAPK4,MAPKG,MCM3,NFKBIA,NQ01,PIR & \\
\hline CDKNAA & & kinase & $-0,116$ & bias & 4,35E-02 & BUB1, СASP3, CCNA2,FAS,IL18,JAG1,MCM3,SERPINE1,SOD2 & \\
\hline rasaggline & & chemical drug & & & $4,37 E-02$ & FAS,Marcks,RACK1 & \\
\hline NSD2 & 0,462 & enzyme & & & $4,37 E-02$ & ITGB7,JUN,RRAS & \\
\hline KRT14 & & other & & & 4,37E-02 & CXCL14,GJB2,KRT8 & \\
\hline APR-246 & & chemical drug & & & 4,37E-02 & CDB1,GADD45B,VCL & \\
\hline CIDEC & & other & & & $4,37 \mathrm{E}-02$ & CO12,SDC1,SERPINE1 & \\
\hline ethylene glycol tetracetic acid & & chemical reagent & & & 4,37E-02 & BIRC3,CD83,CD86 & \\
\hline NRAA3 & & ligand-dependent nuclear r receptor & & & 4,37E-02 & BIRC3,СEBPA,FBP 1,NQO1 & \\
\hline PLN & & transporter & & & $4,37 \mathrm{E}-02$ & APOA1,RACK1,RALA,RRAS & \\
\hline 25-hydroxycholesterol & & chemical reagent & 1,000 & & 4,37E-02 & FOSL1,LL1B,JUN,NFKBIA & \\
\hline ATF2 & & transcripition regulator & 1,969 & bias & $4,42 E-02$ & BCL2A1,CCNA2, ILRL1, JUN,SOD2 & \\
\hline LIPE & & enzyme & & & 4,46E-02 & CEBPA,CP, ILRLL1,JUP,RUN 2, SDC4 & \\
\hline Bм11 & & transcription regulator & & & 4,59E-02 & FAS,HK2,MCM3,SFN & \\
\hline FOLR1 & & transporter & & & $4,59 \mathrm{E}-02$ & BASP1,BIRC3,CD9, СLCA1,ST3GAL4 & \\
\hline KLF3 & & transcription regulator & $-1,155$ & bias & $4,59 \mathrm{E}-02$ & 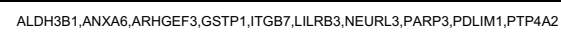 & \\
\hline pregnenolone carbonititie & & chemical drug & & & 4,67E-02 & ALAS1,HMGCS2,UGT1A7 (includes others) & \\
\hline
\end{tabular}




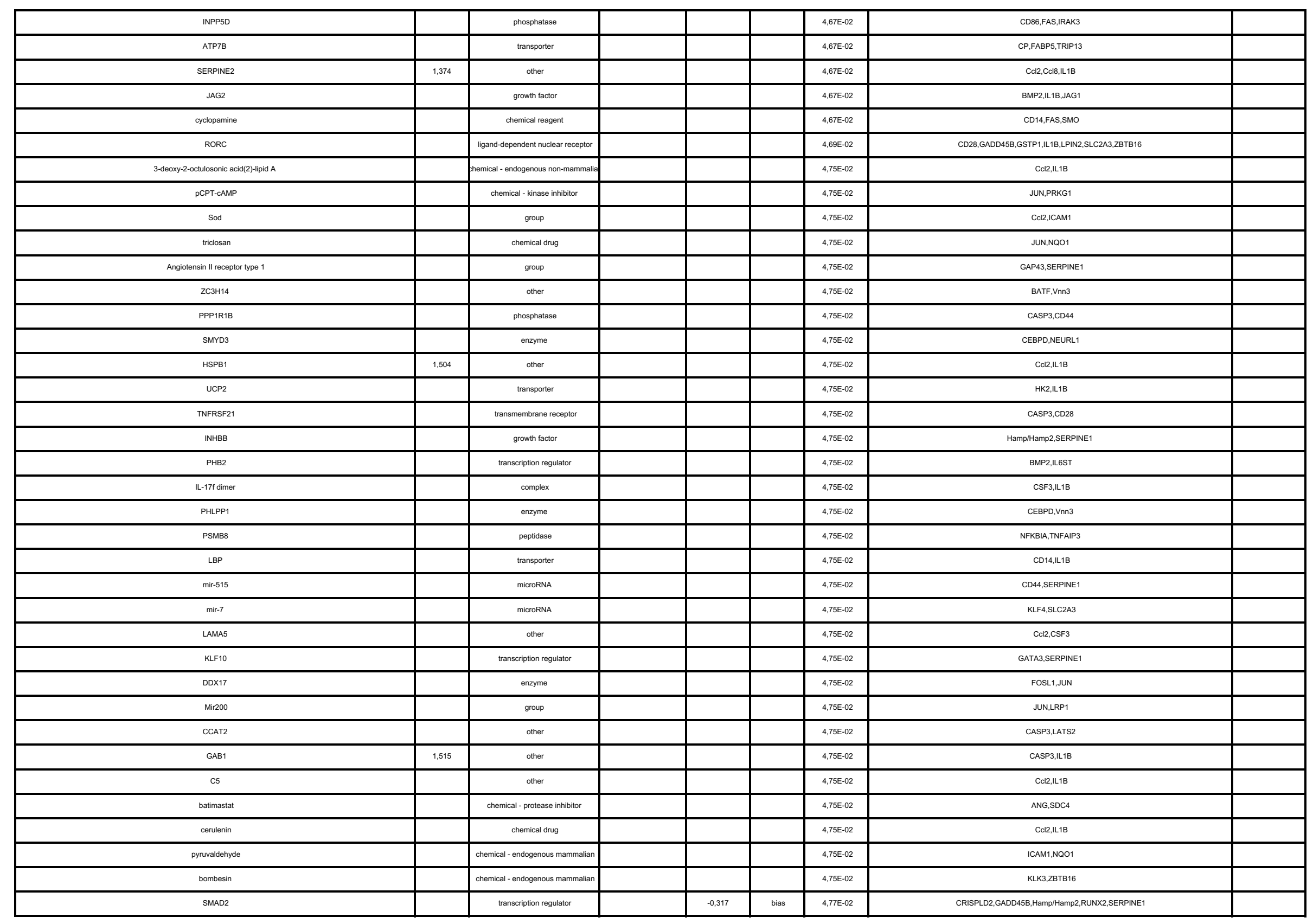




\begin{tabular}{|c|c|c|c|c|c|c|c|}
\hline CDЗE & transmembrane receptor & & & & $4,81 \mathrm{E}-02$ & FAS,JUN,LLP2,NFKBBA & \\
\hline SGK1 & kinase & Activated & 2,000 & bias & $4,81 E-02$ & CEBPA,SLC2A3,SLC3AA2,SMO & \\
\hline sulindac & chemical drug & & & & $4,81 \mathrm{E}-02$ & CCNA2,LL18,NQO1,SERPINB1 & \\
\hline INS & other & & 1,432 & & $4,82 E-02$ & APOA1,CCL1,FADS3,FAS, FOSL 1, CCAM1,JUN & \\
\hline cocaine & chemical drug & Activated & 2,415 & bias & 4,85E-02 & CO12,FOSL1,GADD45B,ICAM1,JUN,LAMC2,MEST,Tpm1 & \\
\hline PXR ligand-PXR-Retinoic acid-RXRa & complex & & & & $4,97 \mathrm{E}-02$ & ALAS1, CYPBA7,UGT1A7 (includes others) & \\
\hline FOXP1 & transcripition regulator & & & & $4,97 E-02$ & DMD,GAP43,JUN & \\
\hline propranolol & chemical drug & & & & $4,97 E-02$ & HК2,IСАM1,NFKBІA & \\
\hline miR-124-3p (and other miRNAs w/seed AAGGCAC) & mature microRNA & Innibitied & $-2,978$ & bias & $5,00 E-02$ & B4GALT1,BTG3,CCL12,CDCA7,CEBPA,F11R,JAG1,LTAF,TSC22D4 & \\
\hline NR3C2 & ligand-dependent nuclear receptor & & 1,954 & & $5,89 E-02$ & 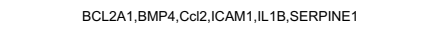 & \\
\hline DNMT3A & enzyme & Innibitied & $-2,000$ & & $2,09 E-01$ & BLNK,IRF5,PLCB4,SDC4,TCFr & \\
\hline
\end{tabular}




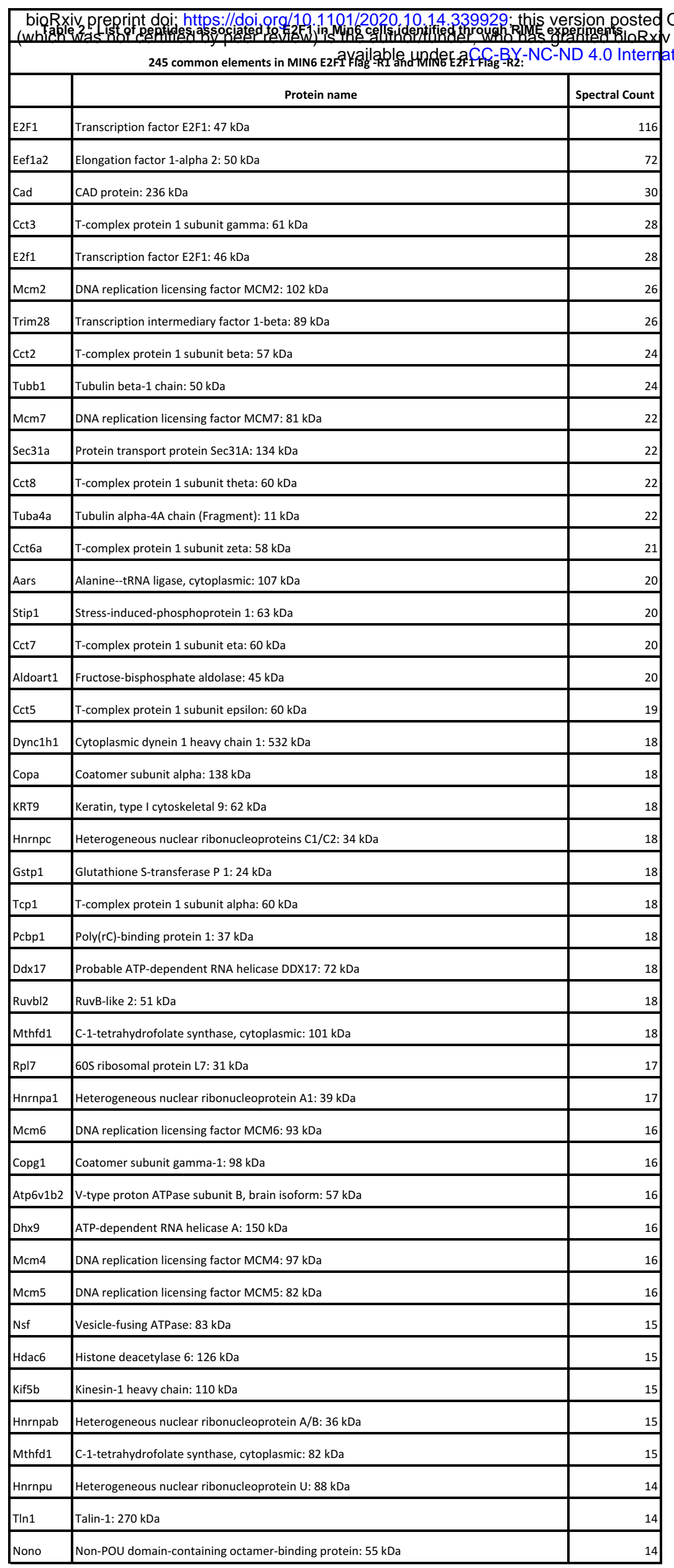




\begin{tabular}{|c|c|c|c|}
\hline 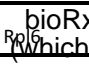 & 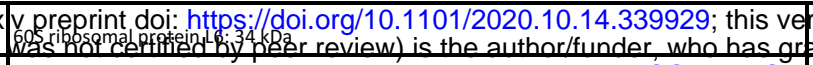 & $\begin{array}{l}\text { sion posted } \\
\text { anted hink }\end{array}$ & $\begin{array}{l}\text { October } 14,2020 \text {. The copyright holder for this preprint } \\
\mathrm{v} \text { a license to display the preprint in perpetuity. It is made }\end{array}$ \\
\hline Arcn1 & Coatomer subunit delta: $57 \mathrm{kDa} \quad$ available under aCC-BY-NC-N & D 4.0 Intern & dational license. \\
\hline Rpl26 & $60 S$ ribosomal protein $L 26: 17 \mathrm{kDa}$ & 14 & \\
\hline Matr3 & Matrin-3: 95 kDa & 14 & \\
\hline Rpl12 & $60 \mathrm{~S}$ ribosomal protein L12: $18 \mathrm{kDa}$ & 14 & \\
\hline Pcbp2 & Poly $(\mathrm{rC})$-binding protein 2: $38 \mathrm{kDa}$ & 14 & \\
\hline Rpl7a & $60 \mathrm{~S}$ ribosomal protein $\mathrm{L7a}: 30 \mathrm{kDa}$ & 14 & \\
\hline Mcm3 & DNA replication licensing factor MCM3: $92 \mathrm{kDa}$ & 13 & \\
\hline Hnrnpl & Heterogeneous nuclear ribonucleoprotein L (Fragment): $67 \mathrm{kDa}$ & 13 & \\
\hline Eif3a & Eukaryotic translation initiation factor 3 subunit A: $162 \mathrm{kDa}$ & 12 & \\
\hline Copb1 & Coatomer subunit beta: $107 \mathrm{kDa}$ & 12 & \\
\hline Rpl8 & 60S ribosomal protein L8: $28 \mathrm{kDa}$ & 12 & \\
\hline Copb2 & Coatomer subunit beta': $102 \mathrm{kDa}$ & 12 & \\
\hline Wdr1 & WD repeat-containing protein $1: 66 \mathrm{kDa}$ & 12 & \\
\hline Sars & Serine--tRNA ligase, cytoplasmic: 61 kDa & 12 & \\
\hline Rps11 & $40 \mathrm{~S}$ ribosomal protein $\mathrm{S} 11: 15 \mathrm{kDa}$ & 12 & \\
\hline Vars & Valine--tRNA ligase: 140 kDa & 12 & \\
\hline Sfpq & Splicing factor, proline- and glutamine-rich: $75 \mathrm{kDa}$ & 12 & \\
\hline Cct4 & T-complex protein 1 subunit delta: $58 \mathrm{kDa}$ & 12 & \\
\hline Ddx1 & ATP-dependent RNA helicase DDX1: $83 \mathrm{kDa}$ & 12 & \\
\hline Ywhab & 14-3-3 protein beta/alpha: $28 \mathrm{kDa}$ & 12 & \\
\hline Rbbp7 & Histone-binding protein RBBP7: $47 \mathrm{kDa}$ & 12 & \\
\hline Rps19 & $40 \mathrm{~S}$ ribosomal protein S19: $16 \mathrm{kDa}$ & 12 & \\
\hline Tubal3 & Tubulin alpha chain-like 3: $50 \mathrm{kDa}$ & 12 & \\
\hline Vcp & Transitional endoplasmic reticulum ATPase: 89 kDa & 11 & \\
\hline Hsp90b1 & Endoplasmin: $92 \mathrm{kDa}$ & 11 & \\
\hline Phgdh & D-3-phosphoglycerate dehydrogenase: $57 \mathrm{kDa}$ & 11 & \\
\hline Ruvbl1 & RuvB-like 1: $50 \mathrm{kDa}$ & 11 & \\
\hline Rbbp4 & Histone-binding protein RBBP4: $48 \mathrm{kDa}$ & 11 & \\
\hline Myo5a & Unconventional myosin-Va: $212 \mathrm{kDa}$ & 11 & \\
\hline Eif4a3 & Eukaryotic initiation factor $4 \mathrm{~A}-111 \mathrm{l}: 47 \mathrm{kDa}$ & 11 & \\
\hline Rrbp1 & Ribosome-binding protein 1: $158 \mathrm{kDa}$ & 10 & \\
\hline Dctn1 & Dynactin subunit 1: $142 \mathrm{kDa}$ & 10 & \\
\hline Lmna & Prelamin-A/C: 74 kDa & 10 & \\
\hline Khsrp & Far upstream element-binding protein 2: $77 \mathrm{kDa}$ & 10 & \\
\hline Rtcb & tRNA-splicing ligase RtcB homolog: 55 kDa & 10 & \\
\hline lars & Isoleucine--tRNA ligase, cytoplasmic: $144 \mathrm{kDa}$ & 10 & \\
\hline Hmgb1 & High mobility group protein B1 (Fragment): $24 \mathrm{kDa}$ & 10 & \\
\hline Cand1 & Cullin-associated NEDD8-dissociated protein 1: $136 \mathrm{kDa}$ & 10 & \\
\hline Lars & Leucine--tRNA ligase, cytoplasmic: 134 kDa & 10 & \\
\hline Hspa9 & Stress-70 protein, mitochondrial: $73 \mathrm{kDa}$ & 10 & \\
\hline Pa2g4 & Proliferation-associated protein 2G4: $44 \mathrm{kDa}$ & 10 & \\
\hline Rpn1 & Dolichyl-diphosphooligosaccharide--protein glycosyltransferase subunit 1: 69 kDa & 10 & \\
\hline Maob & Amine oxidase [flavin-containing] B: $59 \mathrm{kDa}$ & 10 & \\
\hline Rpl28 & $60 S$ ribosomal protein $\mathrm{L} 28: 16 \mathrm{kDa}$ & 10 & \\
\hline Paics & Multifunctional protein ADE2: $47 \mathrm{kDa}$ & 10 & \\
\hline Rpl9 & 60S ribosomal protein L9: $22 \mathrm{kDa}$ & 10 & \\
\hline Cse1l & Exportin-2: 104 kDa & 10 & \\
\hline
\end{tabular}




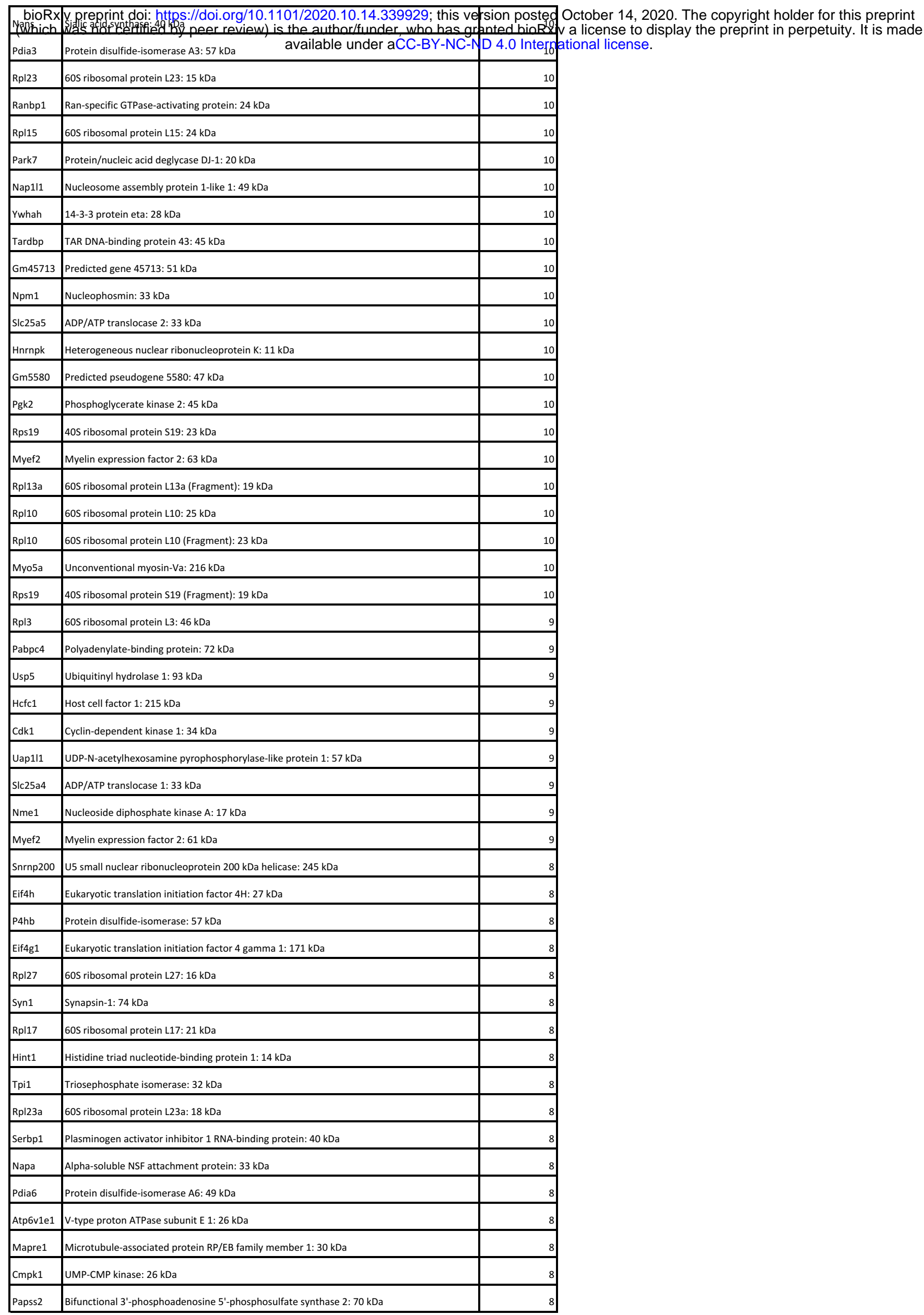




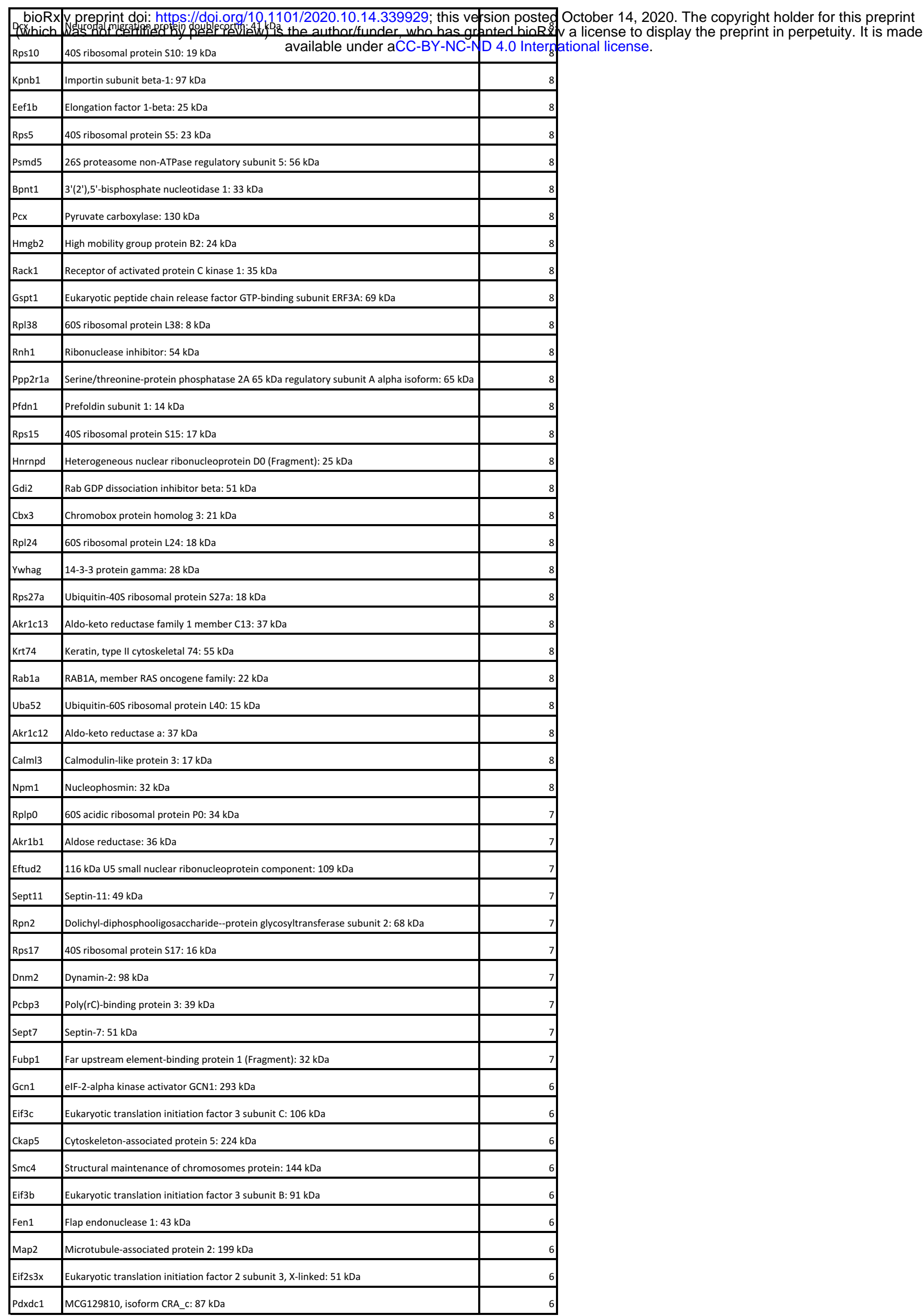




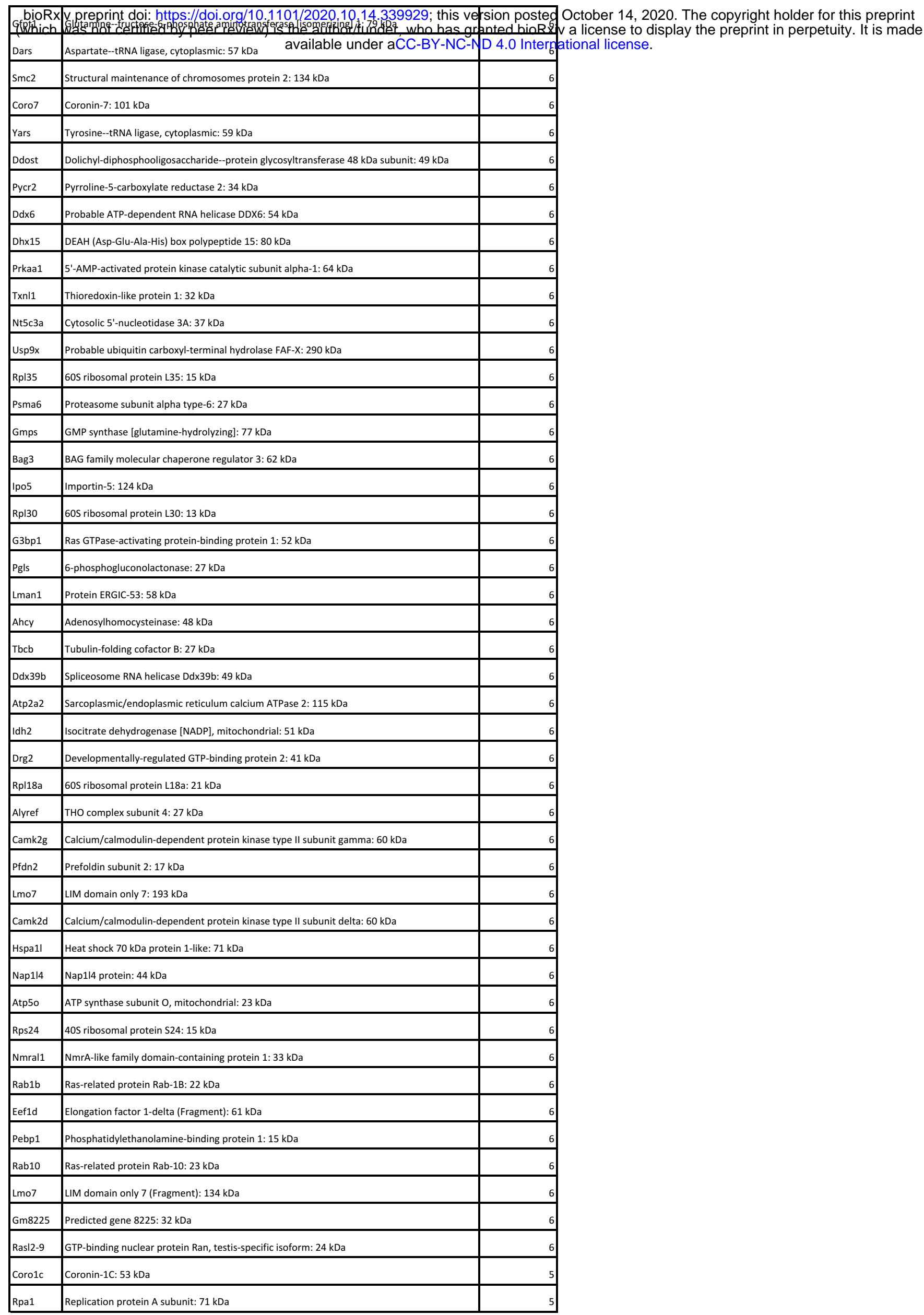




\begin{tabular}{|c|c|c|c|}
\hline 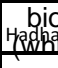 & 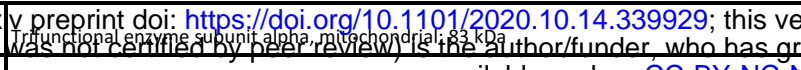 & $\begin{array}{l}\text { sion posted } \\
\text { anted } \mathrm{h} \text { hin }\end{array}$ & $\begin{array}{l}\text { October } 14,2020 \text {. The copyright holder for this preprint } \\
\mathrm{V} \text { a license to display the preprint in perpetuity. It is made }\end{array}$ \\
\hline ckb & Creatine kinase B-type: $43 \mathrm{kDa} \quad$ available under aCC-BY-NC- & D 4.0 Intern & ational license. \\
\hline Gars & Glycine--tRNA ligase: $82 \mathrm{kDa}$ & 5 & \\
\hline Prdx2 & Peroxiredoxin-2: $22 \mathrm{kDa}$ & 5 & \\
\hline Gdi1 & Rab GDP dissociation inhibitor alpha: $51 \mathrm{kDa}$ & 5 & \\
\hline Hdac1 & Histone deacetylase 1: $55 \mathrm{kDa}$ & 5 & \\
\hline Prdx4 & Peroxiredoxin-4 (Fragment): $26 \mathrm{kDa}$ & 5 & \\
\hline Rps24 & $40 \mathrm{~S}$ ribosomal protein S24: $14 \mathrm{kDa}$ & 5 & \\
\hline
\end{tabular}

